\title{
Embedded contact homology and Seiberg-Witten Floer cohomology III
}

\author{
Clifford Henry TAUBes
}

\begin{abstract}
This is the third of five papers that construct an isomorphism between the embedded contact homology and Seiberg-Witten Floer cohomology of a compact 3-manifold with a given contact 1 -form.
\end{abstract}

57R17; 57R57

\section{Introduction}

This is the third of five papers whose purpose is to prove that the embedded contact homology of a compact, oriented 3-dimensional manifold with contact 1 -form is isomorphic to the manifold's Seiberg-Witten Floer cohomology. This isomorphism theorem is stated formally in the first paper of this series [13]. As described in Section 4 of [13], this isomorphism is obtained using two maps: The first takes generators of the embedded contact homology chain complex to generators of the Seiberg-Witten Floer cochain complex. The second associates an instanton from the Seiberg-Witten Floer cohomology differential to each pseudoholomorphic curve from the embedded contact homology differential. The former map is denoted in Theorem 4.2 of [13] by $\Phi^{r}$ and the latter is denoted in Theorem 4.3 of [13] by $\Psi^{r}$. The maps $\Phi^{r}$ and $\Psi^{r}$ are constructed in the second paper of this series [14]. This paper establishes certain additional properties of these maps. In particular, both the embedded contact homology chain complex and the Seiberg-Witten Floer cochain complex are graded complexes, and Theorem 1.1 below describes how $\Phi^{r}$ affects these respective gradings. Meanwhile, Theorem 1.2 asserts that the contribution to the embedded contact homology differential from a given pseudoholomorphic curve is identical to the contribution from its $\Psi^{r}$ image to the Seiberg-Witten Floer cohomology differential.

\section{1.a Relative gradings and the map $\Phi^{r}$}

The upcoming Theorem 1.1 summarizes what is proved here about the map $\Phi^{r}$. The statement of this theorem requires a four part digression to set the stage. This digression summarizes what is needed from $[13 ; 14]$ to state Theorem 1.1 . 
Part 1 Let $M$ denote the 3-manifold in question and $a$ denote the contact 1 -form. The manifold $M$ is oriented using as volume form $a \wedge d a$. The contact form $a$ is implicitly assumed to be from a certain residual set of such forms, this defined in Part 3 of Section 1.a in [14] and denoted by $\mathcal{N}_{M}$.

Use $K^{-1}$ to denote the oriented 2 -plane bundle $\operatorname{kernel}(a) \subset T M$ with its orientation given by $d a$. The dual bundle, $K$, will be viewed for the most part as a complex line bundle over $M$. The first class of $K$ in $H^{2}(X ; \mathbb{Z})$ is denoted by $c_{1}(K)$.

In what follows, $v$ denotes the vector field on $M$ that generates the kernel of $d a$ and pairs with $a$ so as to equal 1 . Its closed integral curves are the Reeb orbits. They are oriented implicitly by $v$. Fix a homology class $\Gamma$ in $H_{1}(M ; \mathbb{Z})$ and let $\mathcal{Z}$ denote the set defined as follows: An element $\Theta \in \mathcal{Z}$ consists of a finite set of pairs of the form $(\gamma, m)$ with $\gamma$ a Reeb orbit and $m$ a positive integer. Require that distinct elements from $\Theta$ have distinct Reeb orbit components, and require that the $\sum_{(\gamma, m) \in \Theta} m \gamma$ define the class $\Gamma$. Given $L \geq 1$, use $\mathcal{Z}^{L}$ to denote the subset consisting of those $\Theta$ with $\sum_{(\gamma, m)} m \ell_{\gamma} \leq L$ where $\ell_{\gamma}$ denotes the integral of the contact 1 -form along $\gamma$. The assumption that $a \in \mathcal{N}_{M}$ guarantees that $\mathcal{Z}^{L}$ is a finite set.

Part 2 Let $p$ denote the greatest integer divisor of the $-c_{1}(K)+2 \mathrm{P}(\Gamma)$, where P: $H_{1}(M ; \mathbb{Z}) \rightarrow H^{2}(M ; \mathbb{Z})$ denotes the Poincaré duality isomorphism. Hutchings [6] explains how the elements in $\mathcal{Z}$ can be given a relative $\mathbb{Z} / p \mathbb{Z}$ grading. There are six steps involved.

Step 1 Fix an almost complex structure, $J$, on $\operatorname{kernel}(a)$ such that $d a(\cdot, J(\cdot))$ defines a Riemannian metric on $\operatorname{kernel}(a)$. Let $\Theta$ denote a given element from $\mathcal{Z}$ and let $(\gamma, m)$ denote a given pair from $\Theta$. There is a disk $D \subset \mathbb{C}$ and an embedding $\varphi: S^{1} \times D \rightarrow M$ with the following properties: First, $\gamma$ appears as $S^{1} \times\{0\}$. Second,

$$
\frac{2 \pi}{\ell_{\gamma}} \varphi * a=\left(1-2 v|z|^{2}-\mu \bar{z}^{2}-\bar{\mu} z^{2}\right) d t+\frac{i}{2}(z d \bar{z}-\bar{z} d z)+\cdots,
$$

$$
\begin{aligned}
\frac{2 \pi}{\ell_{\gamma}} d a & =i d z \wedge d \bar{z}-2(v z+\mu \bar{z}) d \bar{z} \wedge d t-2(v \bar{z}+\bar{\mu} z) d z \wedge d t+\cdots, \\
\frac{\ell_{\gamma}}{2 \pi} v & =\frac{\partial}{\partial t}+2 i(v z+\mu \bar{z}) \frac{\partial}{\partial z}-2 i(v \bar{z}+\bar{\mu} z) \frac{\partial}{\partial \bar{z}}+\cdots .
\end{aligned}
$$

Here, $v$ and $\mu$ are respectively real and complex valued functions on $S^{1}$. The unwritten terms in the top equation are $\mathcal{O}\left(|z|^{3}\right)$ and those in the lower two equations are $\mathcal{O}\left(|z|^{2}\right)$. Here and in what follows, the circle $S^{1}$ is implicitly identified with $\mathbb{R} /(2 \pi \mathbb{Z})$ and $t \in \mathbb{R} /(2 \pi \mathbb{Z})$ is used to denote its affine coordinate. 
Step 2 The pair $(\nu, \mu)$ are used to define the operator $\mathcal{L}$ on $C^{\infty}(\mathbb{R} ; \mathbb{C})$ given by

$$
\mathcal{L} z=\frac{i}{2} \frac{d}{d t} z+v z+\mu \bar{z}
$$

Let $t \rightarrow x(t)$ and $t \rightarrow y(t)$ denote the real and imaginary parts of an element $z(\cdot)$ in the kernel of $\mathcal{L}$. The latter obey

$$
\left(\begin{array}{l}
x(t) \\
y(t)
\end{array}\right)=U\left(\begin{array}{l}
x(0) \\
y(0)
\end{array}\right) \quad \text { where }\left.U\right|_{t} \in \operatorname{SL}(2 ; \mathbb{R}) \text { for each } t \in \mathbb{R} .
$$

As $t$ varies in $[0,2 \pi]$, the map $\left.t \rightarrow U\right|_{t}$ defines a path in $\operatorname{SL}(2 ; \mathbb{R})$ from the identity. The assumption that $a \in \mathcal{N}_{M}$ demands that $\left|\operatorname{trace}\left(\left.U\right|_{2 \pi}\right)\right|>2$ or $\left|\operatorname{trace}\left(\left.U\right|_{2 \pi}\right)\right|<2$. In the former case, $\gamma$ is said to be hyperbolic, and in the latter case $\gamma$ is said to be elliptic. In the hyperbolic case, there is a homotopy of the map $\left.U\right|_{(\cdot)}:[0,2 \pi] \rightarrow \operatorname{SL}(2 ; \mathbb{R})$ such that the $t=2 \pi$ element of each member of this homotopy has $\left|\operatorname{trace}\left(\left.U\right|_{(\cdot)}\right)\right| \geq 2$ and such that the final member is a rotation through $\pi k$ radians with $k \in \mathbb{Z}$. The integer $k$ is said to be the rotation number. In the elliptic case, there is a homotopy of $\left.U\right|_{(\cdot)}$ such that the $t=2 \pi$ element of each member of this homotopy is conjugate to $\left.U\right|_{2 \pi}$ and such that the end member is a rotation by angle $2 \pi R$ with $R \in \mathbb{R}$ an irrational number. The number $\mathrm{R}$ is the rotation number of $\gamma$ when $\gamma$ is elliptic.

Note that $k$ and $\mathrm{R}$ depend on the 1 -jet of $\varphi$. Even so, the hyperbolic/elliptic distinction is intrinsic to $\gamma$. For that matter, so is the $\bmod (2)$ reduction of $k$ when $\gamma$ is hyperbolic, as is the $\bmod (\mathbb{Z})$ reduction of $\mathrm{R}$ when $\gamma$ is elliptic. The point being that a different choice of $\varphi$ can change $k$ by the addition of an even number, and change $\mathrm{R}$ by the addition of an integer.

Step 3 Associate to $\gamma$ and each $q \in\{1,2, \ldots, m\}$ the Conley-Zehnder index; this defined as follows: It is defined to be $q k$ when $\gamma$ is hyperbolic with rotation number $k$. When $\gamma$ is elliptic with rotation number $\mathrm{R}$, the Conley-Zehnder index is equal to 1 plus twice the greatest integer less than $q$ R. In either case, denote the Conley-Zehnder index by $z_{\gamma, q}$. Note that its definition depends on the 1 -jet of $\varphi$ along $\gamma$.

Step 4 Now let $\Theta_{+}$and $\Theta_{-}$denote a pair of elements in $\mathcal{Z}$. Given the homology condition with respect to $\Gamma$, there is an immersed, oriented surface $Z \subset \mathbb{R} \times M$ with the following properties: The $|s| \gg 1$ portion of $Z$ is a disjoint union of embedded cylinders on which $s$ restricts as a function with no critical points. Those cylinders that sit where $s \gg 1$ are labeled in part by the elements in $\Theta_{+}$; a given pair $(\gamma, m)$ labels $m$ such cylinders, each very near the large $|s|$ part of $\mathbb{R} \times \gamma$. To say more, let $\varphi$ denote $\gamma$ 's tubular neighborhood map as described above. Each of these $m$ cylinders sits in $\mathbb{R} \times \varphi\left(S^{1} \times D\right)$ as the image of the graph over $\mathbb{R} \times S^{1}$ of the function that sends $(s, t)$ 
to $e^{-2 \lambda s+i \chi} \in \mathbb{C}$ where $\lambda>0$ and $\chi \in \mathbb{R} /(2 \pi \mathbb{Z})$. However, if $C$ and $C^{\prime}$ are two such cylinders, then the corresponding points $\chi$ and $\chi^{\prime}$ must define distinct points in the circle. There is an analogous labeling of the cylinders that sit where $s \ll-1$ with the elements in $\Theta_{-}$. The only difference in this case is that $\lambda$ is now required to be negative.

The surface $Z$ has a well defined self-intersection number in $(0,1) \times M$; this defined by intersecting $Z$ with a push-off, $Z^{\prime}$, that is defined near $(\gamma, m)$ in $\Theta_{-}$or in $\Theta_{+}$in the same fashion as $Z$. This intersection number is denoted by $Q_{Z}$.

Step 5 The $(\gamma, m)$ be any given element in either $\Theta_{-}$or $\Theta_{+}$. The corresponding may $\varphi$ gives $K$ a section on $S^{1} \times D$, this the $\mathbb{C}$-valued 1 -form $d z$. Define $\left\langle c_{1}, Z\right\rangle$ to be the Euler number of $K$ as defined by counting algebraically the zeros of a section of $\left.K\right|_{Z}$ that is given near any $(\gamma, m)$ in $\Theta_{-}$or $\Theta_{+}$as just described.

Step 6 Introduce now the integer

$$
I\left(\Theta_{-}, \Theta_{+} ; Z\right)=-\left\langle c_{1}, Z\right\rangle+Q_{Z}+\sum_{(\gamma, m) \in \Theta_{+}} \sum_{1 \leq q \leq m} z_{\gamma, q}
$$

$$
-\sum_{(\gamma, m) \in \Theta_{-}} \sum_{1 \leq q \leq m} z_{\gamma, q}
$$

Hutchings [6] proves that the $\bmod (p)$ reduction of $I$ is independent of $Z$, and the choices for the tubular neighborhood maps used for each Reeb orbit involved. The $\bmod (p)$ reduction of $I\left(\Theta_{-}, \Theta_{+} ; Z\right)$ is defined to be $\operatorname{deg}_{\text {ech }}\left(\Theta_{-}\right)-\operatorname{deg}_{\text {ech }}\left(\Theta_{+}\right)$. This difference defines the relative $\mathbb{Z} / p \mathbb{Z}$ grading of the embedded contact homology chain complex. Note that the notation here with $\Theta_{-}$appearing to the left of $\Theta_{+}$as an entry in $I(\cdot, \cdot ; Z)$ is opposite to that taken in [6]. The convention here conforms with that used to define the Seiberg-Witten Floer homology.

Part 3 Let $\Theta \in \mathcal{Z}$ denote a nondegenerate element. Section 1.b in [14] associates a set $\mathfrak{C} \Theta$ and a subset $\mathfrak{C}^{*}$ to $\Theta$. As explained in the three steps that follow, each element in $\mathfrak{C}^{*}$ has an associated integer degree.

Step 1 Focus on a given element $(\gamma, m) \in \Theta$. Introduce the vortex moduli space $\mathfrak{C}_{m}$ as defined in Section 1.b of [14]. The latter consists of equivalence classes of pairs $(A, \alpha)$ with $A$ being a connection on the product $\mathbb{C}$ bundle over $\mathbb{C}$ and with $\alpha$ a 
section of this bundle, thus a $\mathbb{C}$-valued function on $\mathbb{C}$. This pair obeys the equations

- $* F_{A}=-i\left(1-|\alpha|^{2}\right)$.

- $\bar{\partial}_{A} \alpha=0$.

- $|\alpha| \leq 1$.

- The function $\left(1-|\alpha|^{2}\right)$ is integrable on $\mathbb{C}$ and $\int_{\mathbb{C}}\left(1-|\alpha|^{2}\right)=2 \pi m$.

The equivalence relation that defines $\mathfrak{C}_{m}$ has $(A, \alpha) \sim\left(A-u^{-1} d u, u \alpha\right)$ with $u$ any smooth map from $\mathbb{C}$ to $U(1)$.

Step 2 As noted in Section 1.b of [14], this space is the complex manifold $\mathbb{C}^{m}$. It also comes with a natural, complete Kahler metric; but this is not the flat Kahler metric unless $m=1$. In any event, this metric defines a symplectic form and thus the Hamiltonian dynamical system that is defined using the time dependent Hamiltonian function

$$
\hbar=\frac{1}{4 \pi} \int_{\mathbb{C}}\left(2 v|z|^{2}+\left(\mu \bar{z}^{2}+\bar{\mu} z^{2}\right)\right)\left(1-|\alpha|^{2}\right) .
$$

The set $\mathfrak{C}_{(\gamma, m)}$ consists of the maps $\mathfrak{c}: S^{1} \rightarrow \mathfrak{C}_{m}$ that are closed, integral curves of the Hamiltonian vector field defined by $f$; thus solutions to the equation $(i / 2) \mathfrak{c}^{\prime}+$ $\left.\nabla^{(1,0)} \mathfrak{h}\right|_{\mathfrak{c}}=0$, where $\mathfrak{c}^{\prime}$ is shorthand for the $(1,0)$ part of $\mathfrak{c}_{*}(d / d t)$, and where $\nabla^{(1,0)} \mathfrak{h}$ denotes the $(1,0)$ part of the gradient of $h$. Let $\mathfrak{C} \Theta$ denote $\times_{(\gamma, m) \in \Theta} \mathfrak{C}_{(\gamma, m)}$. Thus, any given element in $\mathfrak{C} \Theta$ has the form $\left\{\mathfrak{c}_{\gamma} \in \mathfrak{C}_{(\gamma, m)}\right\}_{(\gamma, m) \in \Theta}$.

Let $\mathfrak{c}: S^{1} \rightarrow \mathfrak{C}_{m}$ denote a given map. Associate to $\mathfrak{c}$ the bundle $\mathfrak{c}^{*} T_{1,0} \mathfrak{C}_{m} \rightarrow S^{1}$. The pullback of the Riemannian connection on $T \mathfrak{C}_{m}$ defines a Hermitian connection on $S^{1}$. The map $\mathfrak{c}$ is said to be nondegenerate when the operator

$$
\xi \rightarrow \frac{i}{2} \nabla_{t} \xi+\left.\left(\nabla_{\xi_{\mathbb{R}}} \nabla^{1.0} h\right)\right|_{\mathfrak{c}}
$$

on $C^{\infty}\left(S^{1} ; \mathfrak{c}^{*} T_{1,0} \mathfrak{C}_{m}\right)$ has trivial kernel. The notation here is such that $\nabla_{t}$ denotes the covariant derivative of the aforementioned Hermitian connection. Also, $\left.\left(\nabla_{\xi_{\mathbb{R}}} \nabla^{1,0} h\right)\right|_{\mathfrak{c}}$ denotes the covariant derivative at $\mathfrak{c}$ along the vector defined by $\xi$ in $\left.T \mathfrak{C}_{m}\right|_{\mathfrak{c}}$ of the vector field $\nabla^{1,0} \mathfrak{h} \in C^{\infty}\left(\mathfrak{C}_{m} ; T_{1,0} \mathfrak{C}_{m}\right)$. The operator in (1-7) is symmetric and elliptic. The spectrum of this operator is a discrete subset of $\mathbb{R}$ with finite multiplicities and no accumulation points. What is denoted here by $\mathfrak{C} \Theta *$ consists of the elements in $\mathfrak{C} \Theta$ of the form $\left\{\mathfrak{c}_{\gamma}\right\}_{(\gamma, m) \in \Theta}$ with all $\mathfrak{c}_{\gamma}$ being nondegenerate.

Step 3 Fix $(\gamma, m) \in \Theta$ and let $\mathfrak{c}_{0}: S^{1} \rightarrow \mathfrak{C}_{m}$ denote the constant map to the symmetric vortex. The latter is the equivalence class of solution to (1-5) with $\alpha^{-1}(0)=0$. Associate to $\mathfrak{c}_{0}$ the operator, $\mathcal{R}$, on $C^{\infty}\left(S^{1} ;\left.T \mathfrak{C}_{m}\right|_{\mathfrak{c}_{0}}\right)$ that is defined as follows: 
- If $\gamma$ is elliptic with rotation number $\mathrm{R}$, then $\mathcal{R}$ is the version of (1-7) that is defined using $\mathfrak{c}_{0}$ and the function $\hbar_{\mathrm{R}}$ given by taking ( $\left.v=\frac{1}{2} \mathrm{R}, \mu=0\right)$ in (1-6).

- If $\gamma$ is hyperbolic with rotation number $k$, then $\mathcal{R}$ is the version of (1-7) that is defined using $\mathfrak{c}_{0}$ and the function $\hbar_{k}$ given by taking $\left(v=\frac{1}{4} k-\frac{1}{2} \mathrm{R}^{\prime}, \mu=0\right)$ in (1-6), with $\mathrm{R}^{\prime}>0$ an irrational number such that $m \mathrm{R}^{\prime} \ll 1$.

Lemma 2.4 in [14] asserts that the operator $\mathcal{R}$ has trivial kernel when $\gamma$ is elliptic, and also trivial kernel when $\gamma$ is hyperbolic and $m=1$.

Suppose that $\mathrm{c}: S^{1} \rightarrow \mathfrak{C}_{m}$ is nondegenerate in the sense that (1-7) with $\hbar$ now defined using (1-6) has trivial kernel. Then the spectral flow from the operator $\mathcal{R}$ to $\mathfrak{c}$ 's version of (1-7) is defined (see, eg Taubes [12]). Use $\operatorname{deg}_{\mathfrak{C}}(\mathfrak{c})$ to denote this spectral flow.

Part 4 The definition of the Seiberg-Witten cochain complex requires the choice of a Riemannian metric on $M$. Such a metric should be chosen so that $* d a=2 a$ and such that $|a|=1$. Such a metric is neither more nor less than an almost complex structure $J$ on the kernel of $a$ that is compatible with $d a$. Indeed, with $J$ chosen, the metric on the kernel of $a$ is given by $d a(\cdot, J(\cdot))$. The rest of the metric is given by declaring that the Reeb vector field is orthogonal to the kernel of $a$ and has norm 1.

Fix such a metric. As noted in Section 3.c of [13], the spinor bundle $\mathbb{S}$ for a given Spin $^{\mathbb{C}}$ structure decomposes as the orthogonal direct sum $E \oplus E K^{-1}$ where $E \rightarrow M$ is a complex, Hermitian line bundle, and where $K$ is now viewed as a complex line bundle. These are the respective $+i$ and $-i$ eigenbundles for the endomorphism given by Clifford multiplication with the contact 1 -form. The first Chern class of $E$ can be used to classify the Spin ${ }^{\mathbb{C}}$ structure. This understood, choose $E$ so that its first Chern class is Poincaré dual to $\Gamma$.

Let $\operatorname{Conn}(E)$ denote the space of smooth, Hermitian connections on $E$. The SeibergWitten equations used here require the choice of a real number $r \geq 1$ and a coclosed 1 -form $\mu$. The corresponding Seiberg-Witten equations are for a pair $(A, \psi) \in$ $\operatorname{Conn}(E) \times C^{\infty}(M ; \mathbb{S})$ :

$$
\text { - } B_{A}-r\left(\psi^{\dagger} \tau \psi-i a\right)-* d \mu+\frac{1}{2} B_{A_{K}}=0 \text {. }
$$

- $D_{A} \psi=0$.

The notation here is as follows: First, $B_{A}$ denotes the metric Hodge star of the curvature 2-form of $A$. Second, $A_{K}$ is a fixed, connection on $K^{-1}$ with harmonic curvature 2 -form. Third, $D_{A}$ denotes the Dirac operator on $C^{\infty}(M ; \mathbb{S})$ as defined using the metric's Levi-Civita connection and the connection $A_{K}+2 A$ on $\operatorname{det}(\mathbb{S})$. Finally, if $\eta$ and $\xi$ are any given sections of $\mathbb{S}$, then $\eta^{\dagger} \tau \xi$ is the $\mathbb{C}$ valued 1 -form whose metric 
inner product with a 1 -form $b$ is $\eta^{\dagger} \operatorname{cl}(b) \xi$ with $\mathrm{cl}(\cdot)$ here denoting the Clifford multiplication endomorphism.

The group $C^{\infty}(M ; U(1))$ acts on the space of solutions to (1-8) as follows: Suppose that $u$ is a map from $M$ to $U(1)$ and $\mathfrak{c}=(A, \psi)$ is a solution to (1-8). Then $u \mathfrak{c}=$ $\left(A-u^{-1} d u, u \psi\right)$ is also a solution to (1-8). Solutions related in this manner are said to be gauge equivalent. With $\mu$ fixed, and $r \geq 1$ given, the space of gauge equivalence classes of solutions to (1-8) is denoted by $\mathcal{M}^{r}$ in what follows.

The 1 -form $\mu$ that appears in (1-8) is constrained to lie in a certain Banach space of smooth 1 -forms. The latter is denoted by $\Omega$ and is described briefly in Section 3.d of [13]. The norm on this space is called the $\mathcal{P}$-norm; it bounds all of the $C^{k}$ norms. In what follows, $\mu$ is assumed to come from $\Omega$ and have $\mathcal{P}$-norm less than 1 .

Associated to any given pair $\mathfrak{c}=(A, \psi) \in \operatorname{Conn}(E) \oplus C^{\infty}(M ; \mathbb{S})$ is an elliptic, symmetric operator, $\mathfrak{L}_{\mathfrak{c}}$, on $C^{\infty}\left(M ; i T^{*} M \oplus \mathbb{S} \oplus i \mathbb{R}\right)$ that is defined so as to send a section $(b, \eta, \phi)$ to the section with respective $i T^{*} M, \mathbb{S}$ and $i \mathbb{R}$ components

- $* d b-d \phi-2^{-1 / 2} r^{1 / 2}\left(\psi^{\dagger} \tau \eta+\eta^{\dagger} \tau \psi\right)$,

- $D_{A} \eta+2^{1 / 2} r^{1 / 2}(\operatorname{cl}(b) \psi+\phi \psi)$,

- $* d * b-2^{-1 / 2} r^{1 / 2}\left(\eta^{\dagger} \psi-\psi^{\dagger} \eta\right)$.

The pair $\mathfrak{c}=(A, \psi)$ is said to be nondegenerate when the kernel of $\mathfrak{L}_{\mathfrak{c}}$ is trivial.

Fix a pair $\left(A_{E}, \psi_{E}\right) \in \operatorname{Conn}(E) \times C^{\infty}(M ; \mathbb{S})$ for which the $r=1$ version of (1-9) has trivial kernel. Use $\mathfrak{L}_{E}$ to denote the latter operator. Let $\mathfrak{c}=(A, \psi) \in \operatorname{Conn}(E) \times$ $C^{\infty}(M ; \mathbb{S})$ denote a nondegenerate pair as defined by (1-9) for the given value of $r$. Then there is a well defined spectral flow from $\mathfrak{L}_{E}$ to $\mathfrak{L}_{\mathfrak{c}}$. The degree of $\mathfrak{c}$ is, by definition, the $\bmod (p)$ reduction of minus this spectral flow. This $\bmod (p)$ degree is gauge invariant; it is denoted in what follows by $\operatorname{deg}_{\mathrm{SW}}(\mathfrak{c})$. Note also that if $\mathfrak{c}$, $\mathfrak{c}^{\prime} \in \operatorname{Conn}(E) \times C^{\infty}(M ; \mathbb{S})$ are both nondegenerate, then $\operatorname{deg}_{\mathrm{SW}}(\mathfrak{c})-\operatorname{deg}_{\mathrm{SW}}\left(\mathfrak{c}^{\prime}\right)$ does not depend on the choice for $\left(A_{E}, \psi_{E}\right)$. This relative $\mathbb{Z} / p \mathbb{Z}$ degree gives the relative grading for the Seiberg-Witten Floer cochain complex.

With the digression now over, reintroduce $\mathfrak{C}^{*}{ }^{*}$ from Step 2 in Part 2 above and define $\mathcal{C} \mathcal{Z}^{*}$ to be $\left\{\mathfrak{C} \Theta^{*}: \Theta \in \mathcal{Z}\right\}$. Let $\mathfrak{X} \subset \mathcal{C} \mathcal{Z}^{*}$ denote a finite set. Theorem 1.1 of [14] supplies a constant $\kappa \geq 1$ and for all $r \geq \kappa$, an injective map $\Phi^{r}: \mathfrak{X} \rightarrow \mathcal{M}^{r}$. The theorem below says more about $\Phi^{r}$.

Theorem 1.1 Fix a finite set $\mathfrak{X} \subset \mathfrak{C} \mathcal{Z}^{*}$. The constant $\kappa \geq 1$ from Theorem 1.1 in [14] can be chosen so that when $r \geq \kappa$, then the following is true: Let $\Phi^{r}: \mathfrak{X} \rightarrow \mathcal{M}^{r}$ 
denote the map from Theorem 1.1 in [14]. If $\chi \in \mathfrak{X}$, then $\Phi^{r}(\chi)$ is nondegenerate. If $\mathfrak{X}_{-}, \mathfrak{X}_{+} \in \mathfrak{X}$, then

$$
\begin{aligned}
\operatorname{deg}_{\mathrm{SW}}\left(\Phi^{r}\left(\chi_{+}\right)\right)-\operatorname{deg}_{\mathrm{SW}}\left(\Phi^{r}\left(\chi_{-}\right)\right) & \\
= & \operatorname{deg}_{\mathrm{ech}}\left(\Theta_{-}\right)-\operatorname{deg}_{\mathrm{ech}}\left(\Theta_{+}\right)+\sum_{\mathfrak{c} \in \chi_{-}} \operatorname{deg}_{\mathfrak{C}}(\mathfrak{c})-\sum_{\mathfrak{c} \in \chi_{+}} \operatorname{deg}_{\mathfrak{C}}(\mathfrak{c}) .
\end{aligned}
$$

This theorem is proved in the upcoming Section 2 of this paper.

Theorem 4.2 in [13] refers to a map E: $\operatorname{Conn}(E) \rightarrow \mathbb{R}$. If it is the case that $\Phi^{r}$ is surjective onto the $\mathrm{E}<2 \pi L$ subset of $\mathcal{M}^{r}$, then Theorem 4.2 in [13] follows directly using Theorem 1.1 here with Theorem 1.1 and Lemmas 2.1-2.4 from [14].

\section{1.b The map $\Psi^{r}$ and the (co)chain complex differentials}

The upcoming Theorem 1.2 summarizes what is proved here about the map $\Psi^{r}$ that appears in Theorem 1.2 of [14]. A digression is also needed to set the stage for this theorem about $\Psi^{r}$. This digression has six parts.

Part 1 The definition of the differential for the embedded contact homology chain complex requires the choice of an almost complex structure from a certain set, $\mathcal{J}_{a}$, of almost complex structures for $T(\mathbb{R} \times M)$. This set is described in [7]. Fix $J \in \mathcal{J}_{a}$. Note that $J$ is unchanged by the constant translations along the $\mathbb{R}$ factor of $\mathbb{R} \times M$. It also maps $\partial / \partial s$ to $v$ and it maps $K^{-1}$, the kernel of $a$, to itself. Moreover, $d a(\cdot, J(\cdot))$ is a Riemannian metric on the kernel of $a$.

Use $\mathcal{Z}_{\text {ech }}$ in what follows to denote the subset of elements in $\mathcal{Z}$ that lack pairs of the form $(\gamma, m)$ with $\gamma$ hyperbolic and $m>1$. Fix two elements, $\Theta_{-}, \Theta_{+} \in \mathcal{Z}_{\text {ech }}$ and define $\mathcal{M}_{1}\left(\Theta_{-}, \Theta_{+}\right)$as follows: An element $\Sigma \in \mathcal{M}_{1}\left(\Theta_{-}, \Theta_{+}\right)$consists of a finite set of pairs of the form $(C, m)$ where $m$ is a positive integer and where $C$ is a $J$-pseudoholomorphic submanifold. These pairs are further constrained as follows: First, distinct pairs have distinct submanifold components. Second, $m=1$ unless $C$ is $\mathbb{R}$ invariant, thus of the form $\mathbb{R} \times \gamma$ with $\gamma \subset M$ a Reeb orbit. To state the third property, let $\pi: \mathbb{R} \times \mathrm{M} \rightarrow M$ denote the projection. Here is the third property: The formal sum $\sum_{(C, m) \in \Sigma} m \pi(C)$ defines a 2-cycle whose boundary is $\sum_{(\gamma, m) \in \Theta_{+}} m \gamma-\sum_{(\gamma, m) \in \Theta_{-}} m \gamma$. Finally, this cycle is homologous rel boundary to the image of a submanifold $Z \subset \mathbb{R} \times M$ of the sort described in Part 2 of Section 1.a for which $I\left(\Theta_{-}, \Theta_{+}, Z\right)$ is defined and equal to 1 . Note that $I\left(\Theta_{-}, \Theta_{+}, \cdot\right)$ assigns the same value to manifolds $Z$ and $Z^{\prime}$ if $\pi(Z)-\pi\left(Z^{\prime}\right)$ defines the boundary of a 3-cycle.

Hutchings proves in [6] that $\mathcal{M}_{1}\left(\Theta_{-}, \Theta_{+}\right)$has the structure of a 1-dimensional manifold with a finite set of components. Moreover, each component is a copy of $\mathbb{R}$, 
this the orbit of any one of its members under the action of the group $\mathbb{R}$ that is induced by latter's action on $\mathbb{R} \times M$ as the constant translations of the $\mathbb{R}$ factor.

With $L>1$ fixed, use $\mathcal{Z}_{\text {ech }}^{L}$ to denote the subset of $\mathcal{Z}_{\text {ech }}$ that consists of elements $\Theta$ with $\sum_{(\gamma, m) \in \Theta} m \ell_{\gamma}<L$.

Part 2 The embedded contact homology chain complex is the free $\mathbb{Z}$ module generated by equivalence classes of pairs of the form $(\Theta, \mathfrak{o})$ where $\Theta \in \mathcal{Z}_{\text {ech }}$ and $\mathfrak{o}$ is an ordering of the set of pairs in $\Theta$ of the form $(\gamma, 1)$ with $\gamma$ a hyperbolic Reeb orbit with even rotation number. The equivalence relation has $(\Theta, \mathfrak{o}) \sim(-1)^{\sigma}\left(\Theta, \mathfrak{o}^{\prime}\right)$ where $\sigma$ is 0 or 1 , this the parity of the permutation that takes $\mathfrak{o}$ to $\mathfrak{o}^{\prime}$.

Let $\left(\Theta_{-}, \mathfrak{o}_{-}\right)$and $\left(\Theta_{+}, \mathfrak{o}_{+}\right)$denote generators of the embedded contact homology chain complex. Hutchings observed that the extra data given by $\mathfrak{o}_{-}$and $\mathfrak{o}_{+}$can be used to orient each component of $\mathcal{M}_{1}\left(\Theta_{-}, \Theta_{+}\right)$. The details of this are provided in Section 9.5 of [8] and summarized in the upcoming Section 3.b. Here is a brief description of how this comes about: Quillen's ideas [11] about determinant line bundles for families of Fredholm line bundles are used, much like in [2], to define what is deemed in Section 9 of [8] to be a coherent system of orientations for $\left\{\mathcal{M}_{1}\left(\Theta_{-}, \Theta_{+}\right)\right\}_{\Theta_{-}, \Theta_{+} \in \mathcal{Z}_{\text {ech }}}$ Such an orientation for any given $\mathcal{M}_{1}\left(\Theta_{-}, \Theta_{+}\right)$is denoted in what follows by $o_{\text {ech }}$.

As an parenthetical remark and for reference in later sections, the relevant operators for the aforementioned version of Quillen's construction are generalizations of the following: Let $C \subset \mathbb{R} \times M$ denote an embedded, pseudoholomorphic curve. Then $C$ has a canonical complex structure, and $C$ 's normal bundle has a complex structure and, as a complex line bundle, a canonical holomorphic structure. Let $N$ denote the latter bundle. Associated to $C$ is an operator $\mathcal{D}_{C}: C^{\infty}(C ; N) \rightarrow C^{\infty}\left(C ; N \otimes T^{0,1} C\right)$ that is defined so as to send a given section $\xi$ of $N$ to

$$
\mathcal{D}_{C} \xi=\bar{\partial} \xi+v_{C} \xi+\mu_{C} \bar{\xi}
$$

where $v_{C}$ is a certain section of $T_{1,0} C$ and $\mu_{C}$ a section of $N^{2} \otimes T^{0,1} C$, these defined by the $1-$ jet along $C$ of the almost complex structure.

As explained in Section 9.5 of [8], the orientation $o_{\mathrm{ech}}$ is used to define the differential for embedded contact homology. What follows briefly summarizes how this is done: The generator of the $\mathbb{R}$ action on $\mathcal{M}_{1}\left(\Theta_{-}, \Theta_{+}\right)$orients any given component. Either this orientation agrees with the previous one or not. If so, assign the component +1 , if not assign the component -1 . Use $\iota_{\text {ech }}$ to denote this sign. Now, write the differential of the generator $\left(\Theta_{+}, \mathfrak{o}_{+}\right)$as a sum of distinct generators with integer coefficients. Suppose that the generator $\left(\Theta_{-}, \mathfrak{o}_{-}\right)$appears in this sum. The coefficient that multiplies $\left(\Theta_{-}, \mathfrak{o}_{-}\right)$is the sum of the versions of $\iota_{\text {ech }}$ that are assigned to the components of $\mathcal{M}_{1}\left(\Theta_{-}, \Theta_{+}\right)$. 
Part 3 Fix $r \geq 1$ and a coclosed 1-form $\mu$ for use in (1-8). The associated SeibergWitten equations on $\mathbb{R} \times M$ are equations for a map, $\mathfrak{d}$, from $\mathbb{R}$ into the space $\operatorname{Conn}(E) \times C^{\infty}(M ; \mathbb{S})$. Write $\mathfrak{d}$ as the map $\left.s \rightarrow(A, \psi)\right|_{s}$ and these equations read

- $\frac{\partial}{\partial s} A+B_{A}-r\left(\psi^{\dagger} \tau \psi-i a\right)-i * d \mu+\frac{1}{2} B_{A_{K}}=0$.

- $\frac{\partial}{\partial s} \psi+D_{A} \psi=0$.

A solution $\mathfrak{d}$ is said to be an instanton when $\{\mathfrak{d}(s)\}_{s \in \mathbb{R}}$ converges as $s \rightarrow-\infty$ and also as $s \rightarrow \infty$, and both limits are solutions to (1-8).

Associated to any given map $\mathfrak{d}=(A, \psi): \mathbb{R} \rightarrow \operatorname{Conn}(E) \times C^{\infty}(M ; \mathbb{S})$ is the elliptic operator, $\mathfrak{D}_{\mathfrak{d}}$, on $C^{\infty}\left(\mathbb{R} \times M ; i T^{*} M \oplus \mathbb{S} \oplus i \mathbb{R}\right)$ that sends a given section $(b, \eta, \phi)$ to the section with respective $i T^{*} M, \mathbb{S}$, and $i \mathbb{R}$ components

- $\frac{\partial}{\partial s} b+* d b-d \phi-2^{-1 / 2} r^{1 / 2}\left(\psi^{\dagger} \tau \eta+\eta^{\dagger} \tau \psi\right)$,

- $\frac{\partial}{\partial s} \eta+D_{A} \eta+2^{1 / 2} r^{1 / 2}(\operatorname{cl}(b) \psi+\phi \psi)$,

- $\frac{\partial}{\partial s} \phi+* d * b-2^{-1 / 2} r^{1 / 2}\left(\eta^{\dagger} \psi-\psi^{\dagger} \eta\right)$.

Here, $d$ denotes the exterior derivative along the $M$ factor of $\mathbb{R} \times M$.

Use $\mathbb{H}$ to denote the Hilbert space completion of the space of compactly supported sections over $\mathbb{R} \times M$ of $i T^{*} M \oplus \mathbb{S} \oplus i \mathbb{R}$ using the norm whose square is defined to be

$$
\|\mathfrak{b}\|_{\mathbb{H}}^{2}=\int_{\mathbb{R} \times M}\left(|\nabla \mathfrak{b}|^{2}+r|\mathfrak{b}|^{2}\right),
$$

where $\nabla$ is the covariant derivative that is defined on sections of $i T^{*} M \oplus \mathbb{S} \oplus i \mathbb{R}$ as follows: View $i T^{*} M \oplus i \mathbb{R}$ as $i T^{*}(\mathbb{R} \times M)$. This done, then $\nabla$ is the Levi-Civita covariant derivative on the $i T^{*}(\mathbb{R} \times M)$ factor. View the connection $A$ as a connection on the pullback $E \rightarrow \mathbb{R} \times M$ and likewise view $A_{K}$ as a connection on $K^{-1} \rightarrow \mathbb{R} \times M$. The latter with the Levi-Civita connection define a covariant derivative for $\mathbb{S} \rightarrow \mathbb{R} \times M$. The associated covariant derivative gives $\nabla$ on the $\mathbb{S}$ factor. Meanwhile, use $\mathbb{L}$ to denote the $L^{2}$ completion of the space of compactly supported sections over $\mathbb{R} \times M$ of $i T^{*} M \oplus \mathbb{S} \oplus i \mathbb{R}$.

If the spinor component of $\mathfrak{d}$ is bounded, then $\mathfrak{D}_{\mathfrak{d}}$ defines a bounded, linear map from $\mathbb{H}$ to $\mathbb{L}$. If $\mathfrak{d}$ is an instanton, and if both the $\mathfrak{c}_{-}=\left.\lim _{s \rightarrow-\infty} \mathfrak{d}\right|_{s}$ and $\mathfrak{c}_{+}=\left.\lim _{s \rightarrow \infty} \mathfrak{d}\right|_{s}$ versions of (1-9) have trivial kernel, then $\mathfrak{D}_{\mathfrak{d}}$ in this context is Fredholm. In the latter case, $\mathfrak{d}$ is said to be nondegenerate when the cokernel of this Fredholm map is trivial.

Part 4 Suppose that $\mathfrak{c}_{-}$and $\mathfrak{c}_{+}$are nondegenerate solutions to (1-8). Let $\mathcal{M}_{1}\left(\mathfrak{c}_{-}, \mathfrak{c}_{+}\right)$ denote the space of instanton solutions to (1-11) with the following properties: First, 
the $s \rightarrow-\infty$ limit of $\mathfrak{d}$ is $\mathfrak{c}_{-}$and the $s \rightarrow \infty$ limit is gauge equivalent to $\mathfrak{c}_{+}$. Second, the Fredholm index of $\mathfrak{D}_{\mathfrak{d}}$ is equal to 1 . Note that this set depends only on the gauge equivalence classes of $\mathfrak{c}_{-}$and $\mathfrak{c}_{+}$in the following sense: Suppose that $u \in C^{\infty}(M ; U(1))$. If $\mathfrak{d}=(A, \psi) \in \mathcal{M}_{1}\left(\mathfrak{c}_{-}, \mathfrak{c}_{+}\right)$, then $u \mathfrak{d}=\left(A-u^{-1} d u, u \psi\right) \in$ $\mathcal{M}_{1}\left(u \mathfrak{c}_{-}, \mathfrak{c}_{+}\right)$.

If $\mathfrak{d} \in \mathcal{M}_{1}\left(\mathfrak{c}_{-}, \mathfrak{c}_{+}\right)$is nondegenerate, then $\mathfrak{d}$ 's component in $\mathcal{M}_{1}\left(\mathfrak{c}_{-}, \mathfrak{c}_{+}\right)$is a smooth 1 -dimensional manifold with a free $\mathbb{R}$ action that is induced by $\mathbb{R}$ 's action on $\mathbb{R} \times M$ as the group of constant translations along the $\mathbb{R}$ factor. As a consequence, all instantons in $\mathfrak{d}$ 's component are also nondegenerate. If $\mathcal{M}_{1}\left(\mathfrak{c}_{-}, \mathfrak{c}_{+}\right)$contains solely nondegenerate elements, then it has a finite set of components. All this is explained in the bible on the subject of Seiberg-Witten Floer homology, Kronheimer and Mrowka [9].

If $\mathfrak{c} \in \mathcal{M}^{r}$ is nondegenerate, then there are versions of the Seiberg-Witten Floer cohomology cochain complex whereby $\mathfrak{c}$ labels a generator. If $\mathfrak{c}_{-}$and $\mathfrak{c}_{+}$are both nondegenerate elements in $\mathcal{M}^{r}$, and if $\mathcal{M}_{1}\left(\mathfrak{c}_{-}, \mathfrak{c}_{+}\right)$consists of solely nondegenerate instantons, then there are versions of the Seiberg-Witten cochain complex where $\mathfrak{c}_{-}$ and $\mathfrak{c}_{+}$label generators; and where the elements in $\mathcal{M}_{1}\left(\mathfrak{c}_{-}, \mathfrak{c}_{+}\right)$are used to compute the integer that multiplies $\mathfrak{c}_{-}$when writing the coboundary of $\mathfrak{c}_{+}$in terms of these generators. This is explained in Chapter 20 of [9]. A very brief summary what is said there is given next in Parts 5 and 6 of this digression.

Part 5 Let $\mathfrak{c}_{-}$and $\mathfrak{c}_{+}$for the moment denote a given pair of nondegenerate elements in $\operatorname{Conn}(E) \times C^{\infty}(M ; \mathbb{S})$. Use $\mathfrak{P}=\mathfrak{P}\left(\mathfrak{c}_{-}, \mathfrak{c}_{+}\right)$to denote the space of piecewise differentiable maps from $\mathbb{R}$ to $\operatorname{Conn}(E) \times C^{\infty}(M ; \mathbb{S})$ that have $s \rightarrow-\infty$ limit that is gauge equivalent to $\mathfrak{c}_{-}$and $s \rightarrow \infty$ limit that is gauge equivalent to $\mathfrak{c}_{+}$. Each $\mathfrak{d} \in \mathfrak{P}$ has its corresponding version of $\mathfrak{D}_{\mathfrak{d}}$ as given in (1-11); but now viewed as a Fredholm operator mapping $\mathbb{H}$ to $\mathbb{L}$. Quillen [11] showed (in a somewhat different context) how such operators define a real line bundle, $\operatorname{det}(\mathfrak{D}) \rightarrow \mathfrak{P}$. If $\mathfrak{d} \in \mathfrak{P}$ is such that either the kernel or cokernel of $\mathfrak{D}_{\mathfrak{d}}$ is nontrivial, then the fiber of $\operatorname{det}(\mathfrak{D})$ at a given $\mathfrak{d} \in \mathfrak{P}$ has a canonical identification with $\wedge^{\max }\left(\operatorname{kernel}\left(\mathfrak{D}_{\mathfrak{d}}\right)\right) \times_{\mathbb{R}}\left(\wedge^{\max } \operatorname{cokernel}\left(\mathfrak{D}_{\mathfrak{d}}\right)\right)^{*}$.

Introduce $\Lambda\left(\mathfrak{c}_{-}, \mathfrak{c}_{+}\right)$to denote the orientation sheaf of $\operatorname{det}(\mathfrak{D})$. As explained in Chapter 20 of [9], this sheaf has the following properties: First, it is suitably gauge invariant and has gauge invariant orientations. Second there is a canonical isomorphism between $\Lambda\left(\mathfrak{c}_{-}, \mathfrak{c}_{+}\right)^{*}$ and $\Lambda\left(\mathfrak{c}_{-}, \mathfrak{c}_{+}\right)$. Third, if $\mathfrak{c}_{0} \in \operatorname{Conn}(E) \times C^{\infty}(M ; \mathbb{S})$ is likewise nondegenerate, then there exists a canonical composition law isomorphism between the modules $\Lambda\left(\mathfrak{c}_{-}, \mathfrak{c}_{0}\right) \otimes_{\mathbb{Z} / 2 \mathbb{Z}} \Lambda\left(\mathfrak{c}_{0}, \mathfrak{c}_{+}\right)$and $\Lambda\left(\mathfrak{c}_{-}, \mathfrak{c}_{+}\right)$.

These properties have the following consequences: Any given nondegenerate element $\mathfrak{c} \in \mathcal{M}^{r}$ has an associated $\mathbb{Z} / 2 \mathbb{Z}$ module, $\Lambda(\mathfrak{c})$, such that if $\mathfrak{c}_{-}$and $\mathfrak{c}_{+}$are any 
two nondegenerate solutions to (1-8), then there is a canonical isomorphism between the modules $\Lambda\left(\mathfrak{c}_{-}\right) \otimes_{\mathbb{Z} / 2 \mathbb{Z}} \Lambda\left(\mathfrak{c}_{+}\right)^{*}$ and $\Lambda\left(\mathfrak{c}_{-}, \mathfrak{c}_{+}\right)$. This understood, a choice of $\mathfrak{o}\left(\mathfrak{c}_{-}\right) \in \Lambda\left(\mathfrak{c}_{-}\right)$and $\mathfrak{o}\left(\mathfrak{c}_{+}\right) \in \Lambda\left(\mathfrak{c}_{+}\right)$defines a unique element in $\Lambda\left(\mathfrak{c}_{-}, \mathfrak{c}_{+}\right)$.

Part 6 Let $\mathcal{M}^{r^{*}} \subset \mathcal{M}^{r}$ denote the subset of nondegenerate elements. A collection

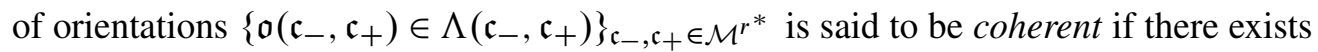
a corresponding set of orientations $\{\mathfrak{o}(c) \in \Lambda(\mathfrak{c})\}_{\mathfrak{c} \in \mathcal{M}^{r}}$ such that any given $\mathfrak{o}\left(\mathfrak{c}_{-}, \mathfrak{c}_{+}\right)$ is equal to $\mathfrak{o}\left(\mathfrak{c}_{-}\right) \mathfrak{o}\left(\mathfrak{c}_{+}\right)$.

The relevance of this orientation business to the problem at hand stems from the following fact: Suppose that $\mathfrak{c}_{-}$and $\mathfrak{c}_{+}$are nondegenerate solutions to (1-8), and suppose that $\mathfrak{d} \in \mathcal{M}_{1}\left(\mathfrak{c}_{-}, \mathfrak{c}_{+}\right)$is also nondegenerate. Then the restriction of $\Lambda\left(\mathfrak{c}_{-}, \mathfrak{c}_{+}\right)$to $\mathfrak{d}$ 's component of $\mathcal{M}_{1}\left(\mathfrak{c}_{-}, \mathfrak{c}_{+}\right)$is canonically isomorphic to the latter's orientation sheaf. With this understood, fix orientations $\{\mathfrak{o}(\mathfrak{c}) \in \Lambda(\mathfrak{c})\}_{\mathfrak{c} \in \mathcal{M}^{r *}}$ so as to define a collection

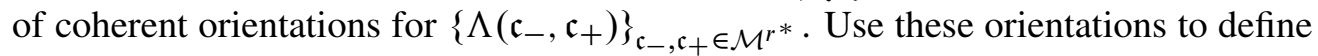
the orientation for the components of $\left\{\mathcal{M}_{1}\left(\mathfrak{c}_{-}, \mathfrak{c}_{+}\right)\right\}_{\mathfrak{c}_{-}, \mathfrak{c}_{+} \in \mathcal{M}^{*}}$ with nondegenerate instantons. This orientation is denoted in what follows by $\mathfrak{o}_{Q}$.

Let $\mathcal{M} \subset \mathcal{M}_{1}\left(\mathfrak{c}_{-}, \mathfrak{c}_{+}\right)$denote a component with nondegenerate instantons. The generator of the $\mathbb{R}$ action on $\mathcal{M}$ also orients $\mathcal{M}$. This orientation is denoted by $\mathfrak{o}_{\mathbb{R}}$. Now view $\mathfrak{c}_{-}$and $\mathfrak{c}_{+}$as generators of the Seiberg-Witten Floer cohomology complex. Then $\mathcal{M}$ contributes +1 to the sum that defines the multiple of $\mathfrak{c}_{-}$in the coboundary of $\mathfrak{c}_{+}$ when ${ }^{\mathfrak{o}} Q=\mathfrak{o}_{\mathbb{R}}$. Otherwise, $\mathcal{M}$ contributes -1 .

With the digression now over, fix $L \geq 1$ and assume the following about the contact 1 -form a and the element $J \in \mathcal{J}_{a}$ :

- There is no element $\Theta \in \mathcal{Z}_{\text {ech }}^{L}$ with $\sum_{(\gamma, m) \in \Theta} m \ell_{\gamma}=L$.

- Suppose that $\gamma$ is a Reeb orbit with $\ell_{\gamma}<L$. Then $\gamma$ has a tubular neighborhood map $\varphi: S^{1} \times D \rightarrow M$ as described in Part 2 of Section 1.a such that if $\gamma$ is hyperbolic with rotation number $k$, then $(\nu, \mu)=$ (1-14) $\quad\left(\frac{1}{4} k, i \varepsilon e^{i k t}\right)$ with $\varepsilon>0$ but very small. Meanwhile, if $\gamma$ is elliptic, then its rotation number $\mathrm{R}$ is irrational. Furthermore,

(i) The pair $(\nu, \mu)=\left(\frac{1}{2} \mathrm{R}, 0\right)$.

(ii) The $\varphi^{*}$-pullback of $T^{1,0}(\mathbb{R} \times M)$ is spanned by $d s+i a$ and $\left(\ell_{\gamma} / 2 \pi\right)(d z-i \mathrm{R} z d t)$.

Fix a 1 -form $\mu \in \Omega$ with $\mathcal{P}$-norm less than 1 for use in (1-8), (1-9) and Theorem 1.1 in [14]. Use the latter theorem to define the large $r$ versions of the map $\Phi^{r}: \mathcal{Z}_{\text {ech }}^{L} \rightarrow \mathcal{M}^{r}$. Use the large $r$ version of Theorem 1.2 in [14] to define the map $\Psi^{r}$ for any given ordered pair $\left(\Theta_{-}, \Theta_{+}\right)$with both elements from $\mathcal{Z}_{\text {ech }}^{L}$. Recall that $\Psi^{r}$ is an injective 
and $\mathbb{R}$-equivariant map from $\mathcal{M}_{1}\left(\Theta_{-}, \Theta_{+}\right)$into the space of instantons with $s \rightarrow-\infty$ limit equal to in the gauge equivalence class $\Phi^{r}\left(\Theta_{-}\right)$and with $s \rightarrow \infty$ limit in the gauge class $\Phi^{r}\left(\Theta_{+}\right)$.

Theorem 1.2 Fix $L \geq 1$ and a pair $(a, J)$ as above that obeys (1-14). There exists $\kappa \geq 1$ with the following significance: Define $\mathcal{M}^{r}$ using $r \geq k$ and the $1-$ form $\mu \in \Omega$

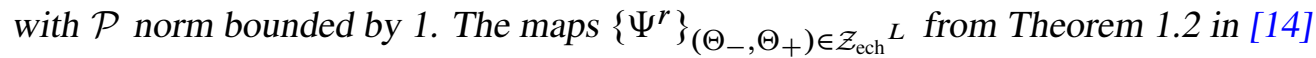
can be constructed so that:

- Let $\Theta_{-}$and $\Theta_{+}$denote any two elements in $\mathcal{Z}_{\text {ech }}^{L}$, and use $\mathfrak{c}_{-}$and $\mathfrak{c}_{+}$to denote solutions to (1-8) from their respective images in $\mathcal{M}^{r}$ via the map $\Phi^{r}$.

(i) The image via $\Psi^{r}$ of $\mathcal{M}_{1}\left(\Theta_{-}, \Theta_{+}\right)$lies in $\mathcal{M}_{1}\left(\mathfrak{c}_{-}, \mathfrak{c}_{+}\right)$and it contains only nondegenerate instantons. In particular, its image consists of a union of smooth components of $\mathcal{M}_{1}\left(\mathfrak{c}_{-}, \mathfrak{c}_{+}\right)$.

(ii) The differential of $\Psi^{r}$ maps the generator of the $\mathbb{R}$ action on $\mathcal{M}_{1}\left(\Theta_{-}, \Theta_{+}\right)$ to the generator of the $\mathbb{R}$-action on its image in $\mathcal{M}_{1}\left(\mathfrak{c}_{-}, \mathfrak{c}_{+}\right)$.

- There is a coherent orientation for the collection $\left\{\Lambda\left(\mathfrak{c}_{-}, \mathfrak{c}_{+}\right)\right\}_{\mathfrak{c}_{-}, \mathfrak{c}_{+} \in \mathcal{M}^{r *} \text { with }}$ the following property: Let $\left(\Theta_{-}, \mathfrak{o}_{-}\right)$and $\left(\Theta_{+}, \mathfrak{o}_{+}\right)$denote any two generators of $\mathcal{C}_{\text {ech }}{ }^{L}$. Use $\mathfrak{c}_{-}$and $\mathfrak{c}_{+}$to denote solutions to (1-8) from the respective $\Phi^{r}$ images of $\Theta_{-}$and $\Theta_{+}$in $\mathcal{M}^{r}$. The pushforward via $\Psi^{r}$ of the orientation $o_{\mathrm{ech}}$ agrees on its image with that defined by the coherent orientation for $\Lambda\left(\mathfrak{c}_{-}, \mathfrak{c}_{+}\right)$.

This theorem is proved in Section 3 of what follows.

Given that the map $\Psi^{r}$ is surjective onto $\mathcal{M}_{1}\left(\mathfrak{c}_{-}, \mathfrak{c}_{+}\right)$, then Theorem 4.3 in [13] follows directly from Theorem 1.2 here and Theorem 1.2 in [14]. The fact that the large $r$ versions of $\Psi^{r}$ are surjective is proved in the final paper of this series [15].

\section{1.c Notation and conventions}

The notation used in what follows comes mostly from [14] where the maps $\Phi^{r}$ and $\Psi^{r}$ are constructed. This said, the reader should be familiar with this reference.

As in $[13 ; 14]$, it is always the case that $c_{0}$ denotes a constant that is greater than 1 and is independent of what ever relevant data is under consideration. The precise value of $c_{0}$ can increase between subsequent appearances.

As in [14], it is useful to have chosen a fixed "bump" function on $\mathbb{R}$; this is a chosen smooth function $\chi: \mathbb{R} \rightarrow[0,1]$ that equals 1 on $\left(-\infty, \frac{5}{16}\right]$ and value 0 on $\left[\frac{7}{16}, \infty\right)$.

An index for the notation used in what follows is provided in the last section of the article, just prior to the references.

Acknowledgements This work was supported in part by the National Science Foundation. 


\section{The proof of Theorem 1.1}

Fix an element $\Theta \in \mathcal{Z}$ and then a collection $\left\{\mathfrak{c}_{\gamma}: S^{1} \rightarrow \mathfrak{C}_{m}\right\}(\gamma, m) \in \Theta$ where any given $\mathfrak{c}_{\gamma}$ is a nondegenerate solution to $\gamma$ 's version of the equation $(i / 2) \mathfrak{c}^{\prime}+\left.\nabla^{(1,0)} \mathfrak{h}\right|_{\mathfrak{c}}=0$. This data comprises an element, $\chi \in \mathfrak{C} \Theta^{*}$. If $r \geq c_{0}$, then Theorem 1.1 in [13] supplies a solution, $\Phi^{r}(\chi) \in \operatorname{Conn}(E) \times C^{\infty}(M ; \mathbb{S})$, to $(1-8)$ with the latter defined with some given choice for $\mu \in \Omega$ with $\mathcal{P}$-norm bounded by 1 . Section 2.a proves that $\Phi^{r}(\chi)$ is a nondegenerate solution to (1-8).

Let $\chi_{-}$and $\mathcal{X}_{+}$denote two given elements in $\mathcal{C Z}^{*}$. Section 2.d proves Theorem 1.1's assertion about their relative degree.

\section{2.a Nondegeneracy}

Define $\chi$ as above and introduce $\mathfrak{c}(\chi)=(A, \psi)$ to denote $\Phi^{r}(\chi)$. This pair is written as $\left(A_{\mathfrak{J}}, \psi_{\mathfrak{J}}\right)+\left((2 r)^{1 / 2} b_{\mathfrak{J}}, \eta_{\mathfrak{J}}\right)$ where notation is used is as follows: First, $\mathfrak{J}$ here denotes the set $\left\{\left(\mathfrak{c}_{\gamma}, \xi_{\gamma}\right)\right\}_{(\gamma, m) \in \Theta}$ where any given $(\gamma, m) \in \Theta$ version of $\xi_{\gamma}$ is the section over $S^{1}$ of $\mathfrak{c}_{\gamma}{ }^{*} T_{1,0} \mathfrak{C}_{m}$ that is described in Lemma 3.10 of [14]. Second, $\left(A_{\mathfrak{J}}, \psi_{\mathfrak{J}}\right)$ is described in Section 3.a of [14]. Third, $\mathfrak{b}_{\mathfrak{J}}=\left(b_{\mathfrak{J}}, \eta_{\mathfrak{J}}, \phi_{\mathfrak{J}}\right)$ is what Lemma 3.10 in [14] denotes as $\mathfrak{b}(\xi)$ for $\xi$ the point in $\times_{(\gamma, m) \in \Theta} L_{1}^{2}\left(S^{1} ; \mathfrak{c}_{\gamma}{ }^{*} T_{1,0} \mathfrak{C}_{m}\right)$ with components $\left(\xi_{\gamma}\right)_{(\gamma, m) \in \Theta}$. This $\mathfrak{b}_{\mathfrak{J}}$ is a section of the bundle $i T^{*} M \oplus \mathbb{S} \oplus i \mathbb{R}$; it obeys an equation that has the schematic form

$$
\mathfrak{L}_{\mathfrak{c}_{\Theta \mathfrak{J}}} \mathfrak{b}+r^{1 / 2} \mathfrak{b} * \mathfrak{b}=\mathfrak{v},
$$

where $\mathfrak{c}_{\Theta \mathfrak{J}}$ denotes $\left(A_{\mathfrak{J}}, \psi_{\mathfrak{J}}\right)$ and where $\mathfrak{v}$ is described in Section 3.c of [14]. The bilinear map $\left(\mathfrak{b}_{1}, \mathfrak{b}_{2}\right) \rightarrow \mathfrak{b}_{1} * \mathfrak{b}_{2}$ has respective $i T^{*} M, \mathbb{S}$ and $i \mathbb{R}$ components

$$
\text { - }-(2 \sqrt{2})^{-1}\left(\eta_{1}^{\dagger} \tau \eta_{2}+\eta_{2}^{\dagger} \tau \eta_{1}\right)
$$$$
\text { - } \operatorname{cl}\left(b_{1}\right) \eta_{2}+\operatorname{cl}\left(b_{2}\right) \eta_{1}+\phi_{1} \eta_{2}+\phi_{2} \eta_{1}
$$$$
\text { - }-(2 \sqrt{2})^{-1}\left(\eta_{1}^{\dagger} \eta_{2}-\eta_{2}^{\dagger} \eta_{1}\right) \text {. }
$$

The proof that $\mathfrak{c}(\chi)$ is nondegenerate is done next in ten steps.

Step 1 This step outlines an argument for the assertion that $\mathfrak{c}(\chi)$ is nondegenerate that makes direct use of the various contraction maps that are defined Section 3 of [14]. Steps 2-10 provide a somewhat longer proof whose presentation is justified by its derivation of various inequalities that are used in Paper 4 [15] of this series.

To start the short proof, remark that the operator $\mathfrak{L}_{\mathfrak{c}}$ sends any given section $\mathfrak{f}$ of $i T^{*} M \oplus \mathbb{S} \oplus i \mathbb{R}$ to

$$
\mathfrak{L}_{\mathfrak{c}(x)} \mathfrak{f}=\mathfrak{L}_{\mathfrak{c} \Theta \mathfrak{J}} \mathfrak{f}+2 r^{1 / 2} \mathfrak{b}_{\mathfrak{J}} * \mathfrak{f}
$$


To prove that $\mathfrak{c}(\chi)$ is nondegenerate, it is enough to prove that (2-3) is surjective. Thus, fix a section $\mathfrak{h}$ of $i T^{*} M \oplus \mathbb{S} \oplus i \mathbb{R}$ so as to see if there is a section $\mathfrak{f}$ that obeys $\mathfrak{L}_{\mathfrak{c}(\chi)} \mathfrak{f}=\mathfrak{h}$. Now, fix $\varepsilon \in\left(-r^{-100}, r^{100}\right)$ Let $\mathfrak{v}_{\varepsilon}=\mathfrak{v}+\varepsilon \mathfrak{h}$. Let $\xi=\left(\xi_{\gamma}\right)_{(\gamma, m) \in \Theta}$ denote for the moment a given element in $\times_{(\gamma, m) \in \Theta} L_{1}^{2}\left(S^{1} ; \mathfrak{c}_{\gamma}^{*} T_{1,0} \mathfrak{C}_{m}\right)$ with small norm. Let $\mathfrak{J}$ for the moment denote the data set $\left\{\left(\mathfrak{c}_{\gamma}, \xi_{\gamma}\right)\right\}_{(\gamma, m) \in \Theta}$, and define $\mathfrak{c}_{\Theta \mathfrak{J}}=\left(A_{\mathfrak{J}}, \psi_{\mathfrak{J}}\right)$ as done in Section 3.a of [14]. Now consider first solving (2-1) with $\mathfrak{v}$ replaced by $\mathfrak{v}_{\mathcal{E}}$.

A repeat of what is done in Sections 3.d-3.f of [14] finds a unique element $\xi^{\varepsilon} \in$ $\times_{(\gamma, m) \in \Theta} L_{1}^{2}\left(S^{1} ; \mathfrak{c}_{\gamma}^{*} T_{1,0} \mathfrak{C}_{m}\right)$ with small norm and a section $\mathfrak{v}_{\varepsilon}$ of $i T^{*} M \oplus \mathbb{S} \oplus i \mathbb{R}$ that obey the $\mathfrak{v}_{\varepsilon}$ version of (2-1) and are described by the $\mathfrak{v}_{\varepsilon}$ version of Lemma 3.10 in [14]. The contraction mapping construction of $\mathfrak{b}^{\varepsilon}$ using the $\mathfrak{v}_{\varepsilon}$ versions of Lemmas 3.53.7 in [14] and the contraction mapping construction of $\xi^{\varepsilon}$ using the $\mathfrak{v}_{\varepsilon}$ versions of Lemmas 3.8-3.9 of [14] guarantee that both $\mathfrak{b}^{\varepsilon}$ and $\xi^{\varepsilon}$ vary smoothly with $\varepsilon$ and give at $\varepsilon=0$ the data that is used to define $\Phi^{r}(\chi)$.

Granted this differentiability, note that the derivative at $\varepsilon=0$ of the $\mathfrak{v}_{\varepsilon}$ version of (2-1) can be written as $\mathfrak{L}^{\prime} \mathfrak{f}^{\prime}=\mathfrak{h}$, where $\mathfrak{f}^{\prime}$ is a certain section of $i T^{*} M \oplus \mathbb{S} \oplus i \mathbb{R}$ and $\mathfrak{L}^{\prime}$ is a certain first order elliptic operator. Note in this regard that it follows that the $i T^{*} M$ and $\mathbb{S}$ components of $\mathfrak{L}^{\prime} \mathfrak{f}^{\prime}$ are the same as those of $\mathfrak{L}_{\mathfrak{c}(x)} \mathfrak{f}^{\prime}$. This follows from (2-2). However, it is not necessarily the case that the $i \mathbb{R}$ component of $\mathfrak{L}^{\prime} \mathfrak{f}^{\prime}$ is that of $\mathfrak{L}_{\mathfrak{c}(x)} \mathfrak{f}^{\prime}$.

Let $\mathfrak{J}$ henceforth denote $\left\{\left(\mathfrak{c}_{\gamma}, \xi_{\gamma}\right)\right\}_{(\gamma, m) \in \Theta}$ with $\xi=\times_{(\gamma, m) \in \Theta} \xi_{\gamma}$ now denoting the element in $\times_{(\gamma, m) \in \Theta} L_{1}^{2}\left(S^{1} ; \mathfrak{c}_{\gamma}^{*} T_{1,0} \mathfrak{C}_{m}\right)$ that is used to define $\Phi^{r}(\chi)$. Write $\mathfrak{f}^{\prime}=$ $\left(b^{\prime}, \eta^{\prime}, \phi^{\prime}\right)$. The desired solution to $\mathfrak{L}_{\mathfrak{c}(x)} \mathfrak{f}=\mathfrak{h}$ has the form $\mathfrak{f}=\left(b^{\prime}-(2 r)^{-1 / 2} d u, \eta^{\prime}+\right.$ $\left.u \psi_{\mathfrak{J}}, \phi^{\prime}\right)$ with $u$ an $i \mathbb{R}$ valued function on $M$. An addition to $\mathfrak{f}^{\prime}$ of this sort does not change the $i T^{*} M \oplus \mathbb{S}$ part of $\mathfrak{L}_{\mathfrak{c}(x)} \mathfrak{f}$ by virtue of the fact that $\psi_{\mathfrak{J}}$ obeys the $A=A_{\mathfrak{J}}$ version of the Dirac equation. Meanwhile, there is a unique choice for $u$ that makes the $i \mathbb{R}$ component of $\mathfrak{L}_{\mathfrak{c}(x)} \mathfrak{f}$ equal to the $i \mathbb{R}$ component of $\mathfrak{h}$.

Step 2 Fix $(\gamma, m) \in \Theta$. Introduce from Step 1 in Section 3.a of [14] the open neighborhood $U_{\gamma}$ of $\gamma$. Step 3 in Section 3.b of [14] introduces a pair of 2-dimensional complex vectors spaces that it denotes as $\mathbb{V}_{0}$ and $\mathbb{V}_{1}$. This same part of [14] goes on to describe an isomorphism over $\mathbb{R}$ between $i T^{*} M \oplus \mathbb{S} \oplus i \mathbb{R}$ over $U_{\gamma}$ and $U_{\gamma} \times\left(\mathbb{V}_{0} \oplus \mathbb{V}_{1}\right)$. Let $\chi_{\gamma}$ denote the cut-off function with support on $U_{\gamma}$ that is defined in Step 2 of Section 3.a in [14]. Let $\mathfrak{p}_{\gamma 0}: S^{1} \times \mathbb{C} \rightarrow \mathbb{V}_{1}$ denote the $\xi=0$ version of what is introduced in Step 1 of the proof of Lemma 3.10 in [14]. View $\left(0, \mathfrak{p}_{\gamma 0}\right)$ as a section over $U_{\gamma}$ of $i T^{*} M \oplus \mathbb{S} \oplus i \mathbb{R}$. As such, $\sum_{(\gamma, m) \in \Theta} \chi_{\gamma}\left(0, \mathfrak{p}_{\gamma 0}\right)$ defines a section over $M$ of the this same bundle. Write the $i T^{*} M \oplus \mathbb{S}$ part of this section as $\left(b_{*}, \eta_{*}\right)$. As it turns out, the $i \mathbb{R}$ part of $\mathfrak{p}_{\gamma 0}$ is zero; this can be verified using the description of $\mathfrak{p}_{\gamma 0}$ that is given below just prior to (2-32). 
Let $\left(A^{*}, \psi^{*}\right)$ denote the version of $\left(A_{\mathfrak{J}}, \psi_{\mathfrak{J}}\right)$ that is defined in Section 3.a of [14] using the data $\left\{\left(\mathfrak{c}_{\gamma}, \xi_{\gamma}=0\right)\right\}$. Use $\mathfrak{L}_{*}$ in what follows to denote the version of (1-9) that is defined by $(A, \psi)=\left(A^{*}+2^{1 / 2} r^{1 / 2} b_{*}, \psi^{*}+\eta_{*}\right)$. The operator $\mathfrak{L}_{*}$ is used to prove that $\mathfrak{L}_{\mathfrak{c}(x)}$ has trivial kernel.

Step 3 This step is a digression to remind the reader of some of what is said in Section 2.a of [14] about solutions to the vortex equations. To start, recall that the $(1,0)$ tangent space to any given vortex $\mathfrak{c}=(A, \alpha)$ in $\mathfrak{C}_{m}$ is the vector space of square integrable elements in the kernel of the operator $\vartheta_{\mathfrak{c}}$ on $C^{\infty}(\mathbb{C} ; \mathbb{C} \oplus \mathbb{C})$ that is defined by

$$
\vartheta_{\mathfrak{c}}(q, \varsigma)=\left(\partial q+2^{-1 / 2} \bar{\alpha}_{\varsigma}, \bar{\partial}_{A} \varsigma+2^{-1 / 2} \alpha q\right) .
$$

Use $\operatorname{kernel}\left(\vartheta_{\mathfrak{c}}\right)$ in what follows to denote this vector space of square integrable elements in the kernel of $\vartheta_{\mathfrak{c}}$. The Kahler metric on $\left.T_{1,0} \mathfrak{C}_{m}\right|_{\mathfrak{c}}$ is $\pi^{-1}$ times the $L^{2}$ metric on $\operatorname{kernel}\left(\vartheta_{\mathfrak{c}}\right)$.

Step 4 To start the story on the operators $\mathfrak{L}_{*}$ and $\mathfrak{L}_{\mathfrak{c}(x)}$, introduce, as done in Step 4 of Section 3.b in [14], the orthogonal projection $\Pi_{0}=\Pi_{\xi=0}$ on the Hilbert space $\mathbb{L}=$ $L^{2}\left(M ; i T^{*} M \oplus \mathbb{S} \oplus i \mathbb{R}\right)$. Use $\Pi_{0}$ to split $\mathbb{L}=\mathbb{L}^{\perp} \oplus \Pi_{0} \mathbb{L}$ where $\mathbb{L}^{\perp}=\left(1-\Pi_{0}\right) \mathbb{L}$.

By way of a reminder, an element in $\Pi_{0} \mathbb{L}$ can be written as $t(\theta)$ where $\theta=$ $\left(\theta_{\gamma}\right)_{(\gamma, m) \in \Theta} \in \bigoplus_{(\gamma, m)} L^{2}\left(S^{1} ; \mathfrak{c}_{\gamma}^{*} T_{1,0} \mathfrak{C}_{m}\right)$ and where

$$
t: \bigoplus_{(\gamma, m)} L^{2}\left(S^{1} ; \mathfrak{c}_{\gamma}^{*} T_{1,0} \mathfrak{C}_{m}\right) \rightarrow \Pi_{0} \mathbb{L}
$$

is defined as follows: Step 1 of Section 3.a in [14] uses a small, positive number, $\rho_{*}$, to define the map $\Phi^{r}$. A particular choice is made in (4-8) of [14]. The homomorphism $t(\theta)$ has support on the radius $\rho_{*}$ tubular neighborhoods of the Reeb orbits from $\Theta$. To see what $t$ looks like near such an orbit, fix $(\gamma, m) \in \Theta$ and use the associated coordinate chart in (1-1) to view neighborhood of $\gamma$ as $S^{1} \times D \subset S^{1} \times \mathbb{C}$. View $i T^{*} M \oplus \mathbb{S} \oplus i \mathbb{R}$ as the restriction of the product bundle $\left(S^{1} \times \mathbb{C}\right) \times\left(\mathbb{V}_{0} \oplus \mathbb{V}_{1}\right)$ with $\mathbb{V}_{0}$ and $\mathbb{V}_{1}$ defined in Step 3 of Section 3.b in [14]. Fix $t \in S^{1}$ and an $L^{2}(\mathbb{C} ; \mathbb{C} \oplus \mathbb{C})$ orthonormal basis, $\left\{e_{k}(\cdot)\right\}_{1 \leq k \leq m}$ for $\operatorname{kernel}\left(\vartheta_{\mathfrak{c}_{\gamma(t)}}\right)$, this the $\mathfrak{c}=\left.\mathfrak{c}_{\gamma}\right|_{t}$ version of (2-4). In the context of (1-14), $\mathfrak{c}_{\gamma}$ is the constant map to $\mathfrak{C}_{m}$ and in which case such a basis can taken to be independent of $t \in S^{1}$. In any event, write the components of $\left.\theta_{\gamma}\right|_{t}$ with respect to this basis as $\left\{\theta_{\gamma, k}(t)\right\}_{1 \leq k \leq m}$. Then $t(\theta)$ pulls back to $S^{1} \times \mathbb{C}$ as the map to $\mathbb{V}_{0} \oplus \mathbb{V}_{1}$ with $\mathbb{V}_{1}$ component zero and with $\mathbb{V}_{0}$ component at $(t, z)$ given by

$$
\sum_{1 \leq k \leq m} r_{\gamma}^{1 / 2} \chi\left(|z| / \rho_{*}\right) \theta_{\gamma, k}(t) \frac{1}{\sqrt{\pi}} e_{k}\left(r_{\gamma}^{1 / 2} z\right) .
$$


Note that the homomorphism $t$ is almost an isometry with respect to the $L^{2}$ norm on $\mathbb{L}$ and on the space $\bigoplus_{(\gamma, m)} L^{2}\left(S^{1} ; \mathfrak{c}_{\gamma}^{*} T_{1,0} \mathfrak{C}_{m}\right)$. This is because

$$
t^{\dagger}(t(\theta))=\theta+v(\theta) \quad \text { where }|v(\theta)| \leq c_{0} r^{-1 / 2}|\theta| \text {. }
$$

Moreover, if $\theta \in L_{1}^{2}$, then $\|v(\theta)\|_{L_{1}^{2}} \leq c_{0} r^{-1 / 2}\|\theta\|_{L_{1}^{2}}$. Here and below, subscripts $L^{2}$ and $L_{1}^{2}$ indicate the respective norms of sections of bundles over $S^{1}$.

Any given $\mathfrak{f} \in \mathbb{L}$ is determined by $\mathfrak{f}^{\perp} \in \mathbb{L}^{\perp}$ and $t^{\dagger}(f) \in \bigoplus_{(\gamma, m) \in \Theta} L^{2}\left(S^{1} ; \mathfrak{c}_{\gamma}^{*} T_{1,0} \mathfrak{C}_{m}\right)$. With regard to the latter, let $t^{\dagger}(\mathfrak{f})_{\gamma}$ denote the $(\gamma, m)$ component of $t^{\dagger}(\mathfrak{f})$. Then

$$
\left|t^{\dagger}(\mathfrak{f})_{\gamma}\right| \leq c_{0} r^{1 / 2} \int_{\mathbb{C}} \chi\left(\rho_{*}^{-1}(\cdot)\right) e^{-\sqrt{r}|z| / c_{0}}|\mathfrak{f}| .
$$

This implies what (2-7) also implies: The $L^{2}$ norm of $t^{\dagger}(\mathfrak{f})$ is bounded by $c_{0}\|\mathfrak{f}\|_{2}$. Here, the notation uses $\|\cdot\|_{2}$ and, below, $\|\cdot\|_{p}$ to indicate the $L^{2}$ and $L^{p>2}$ norms for a section of a bundle over $M$.

Use $\mathbb{H}$ now to denote the Hilbert space that is defined as in Step 1 of Section 3.b in [14]. The norm is given in (3-7) of [14]; it is the same as that depicted in (1-13) with the integration domain $M$ rather than $\mathbb{R} \times M$. As noted in Step 4 of Section 3.b in [14], the projection $\Pi_{0}$ maps $\mathbb{H}$ to itself. This understood, write $\mathbb{H}$ as $\mathbb{H}^{\perp} \oplus \Pi_{0} \mathbb{H}$ where $\mathbb{H}^{\perp}$ here denotes the $L^{2}$ orthogonal complement in $\mathbb{H}$ to $\Pi_{0} \mathbb{H}$. An element $\mathfrak{f} \in \mathbb{H}$ is written with respect to this splitting as $\mathfrak{f}=\mathfrak{f}^{\perp}+t(\theta)$.

Step 5 This step and the next consider $\mathfrak{L}_{\mathfrak{c}(x)}-\mathfrak{L}_{*}$. It is proved momentarily that

$$
\begin{aligned}
&\left\|\left(\left(\mathfrak{L}_{\mathfrak{c}(x)}-\mathfrak{L}_{*}\right) \mathfrak{f}\right)^{\perp}\right\|_{2} \leq c_{0}{ }^{-1}\left(r^{-1 / 2}\left\|\mathfrak{f}^{\perp}\right\|_{\mathbb{H}}+\|\theta\|_{L_{1}^{2}}\right) . \\
&\left\|t^{\dagger}\left(\left(\mathfrak{L}_{\mathfrak{c}(x)}-\mathfrak{L}_{*}\right) \mathfrak{f}\right)\right\|_{L^{2}} \leq c_{0}{ }^{-1} r^{-1 / 2}\left(\left\|\mathfrak{f}^{\perp}\right\|_{\mathbb{H}}+\|\theta\|_{L_{1}^{2}} .\right.
\end{aligned}
$$

To start the proof of (2-9), reintroduce the notation from Section 3.f and Lemma 3.10 of [14] so as to write $\mathfrak{b}(\xi)=\mathfrak{b}_{* \xi}+\mathfrak{e}_{\xi}$. Now write $\left(A_{\mathfrak{J}}, \psi_{\mathfrak{J}}\right)=\left(A^{*}, \psi^{*}\right)+$ $\left(2^{1 / 2} r^{1 / 2} b_{\xi}, \eta_{\xi}\right)$ where $\left(A^{*}, \psi^{*}\right)$ is the $\left\{\xi_{\gamma}=0\right\}(\gamma, m) \in \Theta$ analog of $\left(A_{\mathfrak{J}}, \psi_{\mathfrak{J}}\right)$. Use $\mathfrak{t}_{\xi}$ to denote the sum of $\left(b_{\xi}, \eta_{\xi}\right)$ and the $i T^{*} M \oplus \mathbb{S}$ component of $\mathfrak{b}_{* \xi}-\mathfrak{b}_{*}$. Granted this notation,

$$
\left(\mathfrak{L}_{\mathfrak{c}(x)}-\mathfrak{L}_{*}\right) \mathfrak{f}=r^{1 / 2} \mathfrak{t}_{\xi} * \mathfrak{f}+r^{1 / 2} \mathfrak{e} \xi * \mathfrak{f} .
$$

The inequality in (2-9) is proved by establishing suitable bounds on the norms of the two terms that appear on the right hand side of (2-10) as applied first to $\mathfrak{f}^{\perp}$ and then to $t(\theta)$.

The $L^{2}$ norm of $r^{1 / 2} \mathfrak{e} \xi * \mathfrak{f}^{\perp}$ is bounded by

$$
r^{1 / 2}\left\|\mathfrak{e}_{\xi}\right\|_{4}\left\|\mathfrak{f}^{\perp}\right\|_{4} \leq c_{0} r^{1 / 4}\left\|\mathfrak{e}_{\xi}\right\|_{\mathbb{H}}\left\|\mathfrak{f}^{\perp}\right\|_{\mathbb{H}} \leq c_{0} r^{-3 / 4}\left\|\mathfrak{f}^{\perp}\right\|_{\mathbb{H}}
$$


Indeed, this follows using Hölder's inequality with the Sobolev inequality in (3-9) of [14]. Meanwhile,

$$
r^{1 / 2}\left\|\mathfrak{e}_{\xi} * t(\theta)\right\|_{2} \leq c_{0} r\|\theta\|_{L^{\infty}}\left\|\mathfrak{e}_{\xi}\right\|_{2} \leq c_{0} r^{1 / 2}\|\theta\|_{L_{1}^{2}}\|\mathfrak{e} \xi\|_{\mathbb{H}} \leq c_{0} r^{-1 / 2}\|\theta\|_{L_{1}^{2}}
$$

This uses (2-6) to bound $|t(\theta)|$ by $c_{0} r^{1 / 2}$ times the sup norm, $\|\theta\|_{L^{\infty}}$, of $\theta$. Meanwhile a standard Sobolev inequality asserts that $\|\theta\|_{\infty} \leq c_{0}\|\theta\|_{L_{1}^{2}}$. These last two inequalities are consistent with what is claimed in (2-9).

The analysis of $r^{1 / 2} \mathfrak{t}_{\xi} * \mathfrak{f}$ uses the fact that

$$
\left|\mathfrak{t}_{\xi}\right| \leq c_{0}\|\xi\|_{L^{\infty}} \sum_{(\gamma, m) \in \Theta} e^{-\sqrt{r} \operatorname{dist}(\cdot, \gamma) / 100} .
$$

Since the $L^{\infty}$ norm is bounded by its $L_{1}^{2}$ norm, and as the latter is bounded by $c_{0} r^{-1 / 2}$, so $\left|\mathfrak{t}_{\xi}\right|$ is bounded by $c_{0} r^{-1 / 2} \sum_{(\gamma, m) \in \Theta} e^{-\sqrt{r} \operatorname{dist}(\cdot, \gamma) / 100}$. This understood,

$$
r^{1 / 2}\left\|\mathfrak{t}_{\xi} * \mathfrak{f}^{\perp}\right\|_{2} \leq c_{0}\left\|\mathfrak{f}^{\perp}\right\|_{2} \leq c_{0} r^{-1 / 2}\|\mathfrak{f}\|_{\mathbb{H}} .
$$

By the same token, the $L^{2}$ norm of $r^{1 / 2} \mathfrak{t}_{\xi} * t(\theta)$ is bounded by $c_{0}\|t(\theta)\|_{2} \leq c_{0}\|\theta\|_{L^{2}}$. In particular $r^{1 / 2}\left\|\left(\mathfrak{t}_{\xi} * t(\theta)\right)^{\perp}\right\|_{2} \leq c_{0}\|\theta\|_{L^{2}}$.

Note that the bounds given so far prove the first line of (2-9).

Step 6 The $L^{2}$ norm of $r^{1 / 2} t^{\dagger}\left(\mathfrak{t}_{\xi} * t(\theta)\right)$ is bounded by $c_{0}\|\theta\|_{L^{2}}$. This follows from the just described $L^{2}$ bound on $r^{1 / 2} \mathfrak{t}_{\xi} * t(\theta)$. As is explained next, the $L^{2}$ norm is in fact bounded by $c_{0} r^{-1 / 2}\|\theta\|_{L_{1}^{2}}$.

To see how this comes about, it is necessary digress so as to consider in more detail the operation $*$ as depicted in (2-2) near any given Reeb orbit. Start the digression by fixing $(\gamma, m) \in \Theta$. Write $i T^{*} M \oplus \mathbb{S}$ on $U_{\gamma}$ as $U_{\gamma} \times\left(\mathbb{V}_{0} \oplus \mathbb{V}_{1}\right)$ as in Step 3 of Section 3.b in [14]. This done, view a section $\mathfrak{u}$ of $i T^{*} M \oplus \mathbb{S}$ over $U_{\gamma}$ as a map, $\left(u_{0}, u_{1}\right)$, from $U_{\gamma}$ to $\mathbb{V}_{0} \oplus \mathbb{V}_{1}$. Let $\mathfrak{w}=\left(w_{0}, w_{1}\right)$ denote a second section, also written as a map to $\mathbb{V}_{0} \oplus \mathbb{V}_{1}$. In this notation, write

$$
\left(u_{0}, u_{1}\right) *\left(w_{0}, w_{1}\right)=\left(y_{0}, y_{1}\right) .
$$

Then

$$
\left|y_{0}\right| \leq c_{0}\left(\left|u_{0}\right|\left|w_{1}\right|+\left|u_{1}\right|\left|w_{0}\right|\right)+\mathfrak{r}_{0} \text { and }\left|y_{1}\right| \leq c_{0}\left(\left|u_{0}\right|\left|w_{0}\right|+\left|u_{1}\right|\left|w_{1}\right|\right)+\mathfrak{r}_{1},
$$

where $\left|\mathfrak{r}_{0,1}\right| \leq c_{0}|z||\mathfrak{u}||\mathfrak{w}|$.

Granted the preceding, now recall that there are two contributions to $\mathfrak{t}_{\xi}$ : The first is what was written above as $\left(b_{\xi}, \eta_{\xi}\right)$ and the second from the $i T^{*} M \oplus \mathbb{S}$ part of 
$\mathfrak{b}_{* \xi}-\mathfrak{b}_{\xi}$. Both have support only in $\left\{U_{\gamma}\right\}_{(\gamma, m) \in \Theta}$. Written as a map from $U_{\gamma}$ to $\mathbb{V}_{0} \oplus \mathbb{V}_{1}$, the contribution to $\mathfrak{t}_{\xi}$ from $\left(b_{\xi}, \eta_{\xi}\right)$ has the form $\left(w_{0}, w_{1}\right)$ where

$$
\left|w_{0}\right| \leq c_{0}\|\xi\|_{L_{1}^{2}} e^{-\sqrt{r}|z| / 100} \quad \text { and } \quad\left|w_{1}\right| \leq c_{0}\|\xi\|_{L_{1}^{2}}|z| e^{-\sqrt{r}|z| / 100} .
$$

Indeed, this follows from (2-9) in [14]. Meanwhile the contribution to $\mathfrak{t}_{\xi}$ from $b_{* z}-b_{*}$ appears as $\left(w_{0}, w_{1}\right)$ where

$$
\begin{aligned}
& \left|w_{0}\right| \leq c_{0}\left(r^{-1 / 2}\|\xi\|_{L_{1}^{2}}|z| e^{-\sqrt{r}|z| / 100}+r^{-1}\right) . \\
& \left|w_{1}\right| \leq c_{0}\left(r^{-1 / 2}\|\xi\|_{L_{1}^{2}} e^{-\sqrt{r}|z| / 100}+r^{-1}\right) .
\end{aligned}
$$

Given that the $L_{1}^{2}$ norm of $\xi$ is bounded by $c_{0} r^{-1 / 2}$, these last observations with (2-15) and (2-16) imply that

$$
r^{1 / 2}\left\|t^{\dagger}\left(\mathfrak{t}_{\xi} * t(\theta)\right)\right\|_{L^{2}} \leq c_{0} r^{-1 / 2}\|\theta\|_{L_{1}^{2}} .
$$

This last bound plus what was proved earlier about the $\mathfrak{f}^{\perp}$ contributions imply the second line in (2-9).

Step 7 This step derives some fundamental norm inequalities for $\mathfrak{L}_{*} \mathfrak{f}$. To this end, write $\mathfrak{f}=\mathfrak{f}^{\perp}+t(\theta)$. With $\mathfrak{f}$ written this way, the $\mathbb{L}^{\perp}$ component of $\mathfrak{L}_{*} \mathfrak{f}$ and the image of $\mathfrak{L}_{*} \mathfrak{f}$ under $t^{\dagger}$ can be written schematically as

$$
\begin{gathered}
\left(\mathfrak{L}_{*} \mathfrak{f}\right)^{\perp}=\mathfrak{L}^{\perp} \mathfrak{f}^{\perp}+\wp(\theta) . \\
t^{\dagger}\left(\mathfrak{L}_{*} \mathfrak{f}\right)=\wp^{\dagger} \mathfrak{f}^{\perp}+\mathrm{D} \theta .
\end{gathered}
$$

Here, $\mathfrak{L}^{\perp}=\left(1-\Pi_{0}\right) \mathfrak{L}_{*}\left(1-\Pi_{0}\right)$. Meanwhile, D is viewed in what follows as a linear map from $\bigoplus_{(\gamma, m)} L_{1}^{2}\left(S^{1} ; \mathfrak{c}_{\gamma}^{*} T_{1,0} \mathfrak{C}_{m}\right)$ to $\bigoplus_{(\gamma, m)} L^{2}\left(S^{1} ; \mathfrak{c}_{\gamma}^{*} T_{1,0} \mathfrak{C}_{m}\right)$. As such, it is also diagonal and so written as $\mathrm{D}=\left(\mathrm{D}_{\gamma}\right)_{(\gamma, m) \in \Theta}$. By the same token, $\wp$ maps $\bigoplus_{(\gamma, m) \in \Theta} L_{1}^{2}\left(S^{1} ; \mathfrak{c}_{\gamma}^{*} T_{1,0} \mathfrak{C}_{m}\right)$ into $\mathbb{L}^{\perp}$.

What follows addresses the norms of the various terms that appear in (2-19). Start with $\left\|\mathfrak{L}^{\perp} \mathfrak{f}\right\|_{2}$. Given Lemma 3.2 from [14], and given that $\left|\mathfrak{b}_{*}\right| \leq c_{0} r^{-1 / 2}$, it follows that

$$
\left\|\mathfrak{L}^{\perp} \mathfrak{f}^{\perp}\right\|_{2} \geq c_{0}^{-1}\left\|\mathfrak{f}^{\perp}\right\|_{\mathbb{H}} .
$$

Meanwhile, (3-13) and (3-14) in [14] and (2-6) imply that

$$
\|\wp(\theta)\|_{2} \leq c_{0}\|\theta\|_{L_{1}^{2}} .
$$

As for $\wp^{\dagger}\left(\mathfrak{f}^{\perp}\right)$, the argument used in Step 2 of the proof of Lemma 3.8 in [14] has what is in essence a cosmetic modification that proves

$$
\left\|\wp^{\dagger}\left(\mathfrak{f}^{\perp}\right)\right\|_{L^{2}} \leq c_{0} r^{-1 / 2}\left\|\mathfrak{f}^{\perp}\right\|_{\mathbb{H}} .
$$


The next lemma gives the fundamental observation about D.

Lemma 2.1 There exists a constant $\kappa \geq 1$ such that if $r \geq \kappa$, then the operator D in

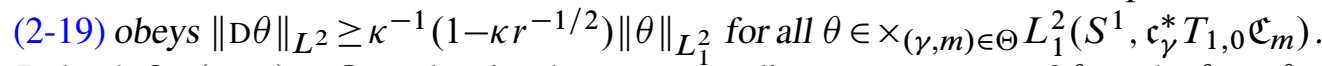
Indeed, fix $(\gamma, m) \in \Theta$ and write the corresponding components of $\theta$ and $\mathrm{D} \theta$ as $\theta_{\gamma}$ and $(\mathrm{D} \theta)_{\gamma}$. Then up to an overall $\gamma$-dependent factor,

$$
(\mathrm{D} \theta)_{\gamma}=\frac{i}{2} \nabla_{t} \theta_{\gamma}+\left.\left(\nabla_{\left(\theta_{\gamma}\right)_{\mathbb{R}}} \nabla^{1,0} \mathfrak{h}\right)\right|_{\mathfrak{c}_{\gamma}}+\mathfrak{e}_{\gamma},
$$

where $\mathfrak{e}_{\gamma}$ is such that $\left\|\mathfrak{e}_{\gamma}\right\|_{L^{2}} \leq c_{0} r^{-1 / 2}\|\theta\|_{L_{1}^{2}}$. Here, as in (1-7), $\nabla_{t}$ denotes the covariant derivative on $\mathfrak{c}_{\gamma}^{*} T_{1,0} \mathfrak{C}_{m}$ and $\left(\theta_{\gamma}\right)_{\mathbb{R}}$ denotes the vector in $T \mathfrak{C}_{m}$ defined by $\theta_{\gamma}$.

This lemma is proved shortly.

Lemma 2.1 with (2-21) and (2-22) imply that

$$
\left\|\mathfrak{L}_{*} \mathfrak{f}\right\|_{2}^{2} \geq c_{0}^{-1}\left(\left\|\mathfrak{f}^{\perp}\right\|_{\mathbb{H}}^{2}+\|\theta\|_{L_{1}^{2}}^{2}\right) .
$$

To see how this comes about, note that (2-22) and Lemma 2.1 imply that

$$
\left\|\mathfrak{L}_{*} \mathfrak{f}\right\|_{2}^{2} \geq c_{0}^{-1}\left\|\mathfrak{L}^{\perp} \mathfrak{f}^{\perp}+\wp(\theta)\right\|_{2}^{2}+c_{0}{ }^{-1}\|\theta\|_{L_{1}^{2}}^{2}-c_{0} r^{-1}\left\|\mathfrak{f}^{\perp}\right\|_{\mathbb{H}}^{2} .
$$

Meanwhile, the right hand side of (2-24) is no less than

$$
\varepsilon c_{0}{ }^{-1}\left\|\mathfrak{L}^{\perp} \mathfrak{f}^{\perp}\right\|_{2}{ }^{2}-\varepsilon\|\wp(\theta)\|_{2}{ }^{2}+c_{0}{ }^{-1}\|\theta\|_{L_{1}^{2}}^{2}-c_{0} r^{-1}\left\|\mathfrak{f}^{\perp}\right\|_{\mathbb{H}}^{2},
$$

where $\varepsilon$ can be any given number in $\left(0, \frac{1}{4}\right)$. What with $(2-20)$ and $(2-21)$, this last inequality implies (2-23).

Step 8 This step finish the proof of that $\mathfrak{L}_{\mathfrak{c}(x)}$ has trivial kernel. To this end, introduce as notation $\mathfrak{o}$ to denote $\mathfrak{L}_{\mathfrak{c}(x)}-\mathfrak{L}_{*}$. Now observe that

$$
\left\|\mathfrak{L}_{\mathfrak{c}(x)} \mathfrak{f}\right\|_{2}^{2} \geq c_{0}^{-1}\left(\left\|\left(\left(\mathfrak{L}_{*}+\mathfrak{o}\right) \mathfrak{f}\right)^{\perp}\right\|_{2}^{2}+\left\|t^{\dagger}\left(\left(\mathfrak{L}_{*}+\mathfrak{o}\right) \mathfrak{f}\right)\right\|_{L_{1}^{2}}^{2}\right) .
$$

What with the last line in (2-9) and (2-20) and Lemma 2.1, this implies that

$$
\left\|\mathfrak{L}_{\mathfrak{c}(x)} \mathfrak{f}\right\|_{2}^{2} \geq c_{0}^{-1}\left\|\left(\left(\mathfrak{L}_{*}+\mathfrak{o}\right) \mathfrak{f}\right)^{\perp}\right\|_{2}^{2}+c_{0}\|\theta\|_{L_{1}^{2}}^{2}-c_{0} r^{-1}\left\|\mathfrak{f}^{\perp}\right\|_{\mathbb{H}}^{2} .
$$

Now write $\left(\mathfrak{L}_{*} f\right)^{\perp}$ as $\mathfrak{L}^{\perp} \mathfrak{f}^{\perp}+\wp(\theta)$ and use the first line of (2-11) to see from (2-27) that

$$
\begin{aligned}
\| \mathfrak{L}_{\mathfrak{c}(x)} \mathfrak{f}_{2}{ }^{2} \geq\left(\varepsilon c_{0}^{-1}\left\|\mathfrak{L}^{\perp} \mathfrak{f}^{\perp}\right\|_{2}{ }^{2}-\varepsilon\|\wp(\theta)\|_{2}{ }^{2}-\varepsilon \|\right. & \left.\|_{L_{1}^{2}}^{2}\right) \\
& +c_{0}\|\theta\|_{L_{1}^{2}}^{2}-c_{0} r^{-1}\left\|\mathfrak{f}^{\perp}\right\|_{\mathbb{H}}^{2}
\end{aligned}
$$


for any $\varepsilon \in\left(0, \frac{1}{8}\right)$. For a suitable $\varepsilon=c_{0}^{-1}$, this last inequality with (2-20) and (2-21) prove that

$$
\left\|\mathfrak{L}_{\mathfrak{c}(x)} \mathfrak{f}\right\|_{2}^{2} \geq c_{0}^{-1}\left(\left\|\mathfrak{f}^{\perp}\right\|_{\mathbb{H}}^{2}+\|\theta\|_{L_{1}^{2}}^{2}\right) .
$$

This with the fact that $t$ is nearly isometric as a map from $\bigoplus_{(\gamma, m) \in \Theta} L^{2}\left(S^{1} ; \mathfrak{c}_{\gamma}^{*} T_{1,0} \mathfrak{C}_{m}\right)$ to $\mathbb{L}$ proves that $\mathfrak{L}_{\mathfrak{c}(x)}$ has trivial kernel when $r \geq c_{0}$.

Step 9 This step and the next contain:

Proof of Lemma 2.1 In order to identify $\mathrm{D} \theta$ and so prove Lemma 2.1, it is necessary to return to (3-13) in [14] for it is the latter equation that gives the D part of $\mathfrak{L}_{*}$. Equation (3-13) in [14] has terms designated as $\mathfrak{r}_{00}$ and $\mathfrak{r}_{01}$; and their $\mathcal{O}(1), \mathcal{O}(|z| \partial)$ and $\mathcal{O}(|z| \bar{\partial})$ make an $\mathcal{O}(1)$ contribution to $\mathrm{D} \theta$. This understood, fix $(\gamma, m) \in \Theta$ and so as to view the $(\gamma, m)$ component of $\mathrm{D}(\cdot)$. A somewhat more detailed analysis rewrites the contribution to the $\left(A^{*}, \psi^{*}\right)$ version of (3-13) in [14] from a section $\mathfrak{f}_{0}=\left(q, \eta_{0}\right)$ of $\mathbb{V}_{0}$ as

$$
\begin{gathered}
2\left(\frac{2 \pi}{\ell_{\gamma}}\right)\left(\frac{i}{2} \frac{\partial}{\partial t} q-(v z+\mu \bar{z}) \frac{\partial}{\partial z} q+(v \bar{z}+\bar{\mu} z) \frac{\partial}{\partial \bar{z}} q+v q+\mu \bar{q}\right)+\mathfrak{r}_{* 0} \\
2\left(\frac{2 \pi}{\ell_{\gamma}}\right)\left(\frac{i}{2} \nabla_{t A_{\gamma}} \eta_{0}-(v z+\mu \bar{z}) \partial_{A_{\gamma}} \eta_{0}+(v \bar{z}+\bar{\mu} z) \bar{\partial}_{A_{\gamma}} \eta_{0}\right)+\mathfrak{r}_{* 1},
\end{gathered}
$$

where $\left|\mathfrak{r}_{* 0}\left(\mathfrak{f}_{0}\right)\right|+\left|\mathfrak{r}_{* 1}\left(\mathfrak{f}_{0}\right)\right| \leq c_{0}\left(|z|\left(\left|\mathfrak{f}_{0}\right|+\left|\nabla_{t} \mathfrak{f}_{0}\right|\right)+|z|^{2}\left|\nabla \mathfrak{f}_{0}\right|\right)$.

Fix $t_{0} \in S^{1}$ and then fix an $L^{2}(\mathbb{C} ; \mathbb{C} \oplus \mathbb{C})$ orthonormal basis, $\left\{e_{k}(\cdot)\right\}_{1 \leq k \leq m}$ for the $\operatorname{kernel}\left(\vartheta_{\mathfrak{c}_{\gamma_{(}(t)}}\right)$ for $t \in S^{1}$ near $t_{0}$. Assume that this basis varies smoothly with $t$. Write the $(\gamma, m)$ component of $\theta$ at points $t \in S^{1}$ near $t_{0}$ in terms of this basis as $\sum_{1 \leq k \leq m} \theta_{\gamma, k}(t) e_{k}$. Likewise, write $\sum_{1 \leq k \leq m}(\mathrm{D} \theta)_{\gamma, k} e_{k}$ for the $(\gamma, m)$ component of $\mathrm{D} \theta$. Now use (2-6) and (2-30) to see that the contribution to $\mathrm{D}$ from (2-30) is given by $2\left(2 \pi / \ell_{\gamma}\right)$ times

$$
\frac{i}{2} \nabla_{t} \theta_{\gamma, k}+v \sum_{1 \leq j \leq m} \theta_{\gamma, j} \frac{1}{\pi} \int_{\mathbb{C}} \bar{\iota}_{k} \iota_{j}+\mu \sum_{1 \leq j \leq m} \bar{\theta}_{\gamma, j} \frac{1}{\pi} \int_{\mathbb{C}} \bar{x}_{k} \bar{x}_{j}+\mathfrak{e},
$$

where the notation is as follows: First, $\nabla_{t}$ denotes the covariant derivative on $\mathfrak{c}_{\gamma}^{*} T_{1,0} \mathfrak{C}_{m}$. Meanwhile, any given pair $\iota_{k}$ and $x_{k}$ are the two components of $e_{k}$. Finally, what is written as $\mathfrak{e}$ obeys $\|\mathfrak{e}\|_{L^{2}} \leq c_{0} r^{-1 / 2}\|\theta\|_{L_{1}^{2}}$.

What is written in (2-31) contributes to $\gamma$ 's component of $\mathrm{D} \theta$, but it is not the end of the story because there is still the contribution to $\mathfrak{L}_{*}$ from $\left(b_{*}, \eta_{*}\right)$. To see the contribution from $\left(b_{*}, \eta_{*}\right)$, recall that $\left(b_{*}, \eta_{*}\right)$ near $\gamma$ is the $i T^{*} M \oplus \mathbb{S}$ portion of 
a map to $\mathbb{V}_{0} \oplus \mathbb{V}_{1}$ that is given by $\left(0, \mathfrak{p}_{\gamma 0}\right)$ with $\mathfrak{p}_{\gamma, 0}$ described as follows: Rescale by $r_{\gamma}^{1 / 2}$ so as to write $\mathfrak{p}_{\gamma 0}(t, z)$ as $v(t) \mathfrak{u}\left(t, r_{\gamma}^{1 / 2} z\right)+\mu(t) \mathfrak{w}\left(t, r_{\gamma}^{1 / 2} z\right)$. Then $\mathfrak{u}$ and $\mathfrak{w}$ obey

$$
\begin{aligned}
\vartheta^{\dagger} \mathfrak{u}+\left(1-\Pi_{0}\right)\left[z\left(2^{-1 / 2}\left(1-|\alpha|^{2}\right), \partial_{A} \alpha\right)\right] & =0, \\
\vartheta^{\dagger} \mathfrak{w}+\left(1-\Pi_{0}\right)\left[\bar{z}\left(2^{-1 / 2}\left(1-|\alpha|^{2}\right), \partial_{A} \alpha\right)\right] & =0,
\end{aligned}
$$

where the notation is as follows: First, $(A, \alpha)$ here denote the vortex $\mathfrak{c}_{\gamma}$. Second, $\vartheta$ is the operator from (2-4) as defined by this same symmetric vortex. Second, $\Pi_{0}$ denotes the projection onto the kernel of $\vartheta$. In the case when $\mathfrak{c}_{\gamma}$ is the symmetric vortex,

$$
\mathfrak{u}=-\left(2^{1 / 2} z \alpha^{-1} \partial_{A} \alpha, 0\right) \text { and } \text { for } m=1, \mathfrak{w}=-\left(0, \bar{z} \bar{\alpha}^{-1}\left(1-|\alpha|^{2}\right)\right) .
$$

In the general case, it is a consequence of (2-7) in [14] and the top line in (1-5) here that $\mathfrak{u}=(y, 0)$ and $\mathfrak{w}=(0, \varsigma)$ where $y$ and $\varsigma$ obey

$$
-\partial \bar{\partial} y+\frac{1}{2}|\alpha|^{2} y=-2^{-1 / 2}\left(1-|\alpha|^{2}\right) \quad \text { and } \quad-\partial_{A} \bar{\partial}_{A} \varsigma+\frac{1}{2} \varsigma=-\partial_{A} \alpha .
$$

The contribution to $(\mathrm{D} \theta)_{\gamma}$ from $\left(b_{*}, \eta_{*}\right)$ adds to what is written in (2-31) so as to give

$$
\begin{aligned}
(\mathrm{D} \theta)_{\gamma}=\frac{i}{2} \nabla_{t} \theta_{\gamma, k} & +v \sum_{1 \leq j \leq m} \theta_{\gamma, j} \frac{1}{\pi} \int_{\mathbb{C}} \bar{\iota}_{k} \iota_{j}\left(1-\frac{1}{\sqrt{2}} y\right) \\
& +\mu \sum_{1 \leq j \leq m} \bar{\theta}_{\gamma, j} \frac{1}{\pi} \int_{\mathbb{C}}\left(\frac{1}{\sqrt{2}} \bar{x}_{k} \bar{x}_{j}-\frac{1}{2} \varsigma\left(\bar{x}_{k} \bar{\iota}_{j}+\bar{x}_{j} \bar{\iota}_{k}\right)\right)+\mathfrak{e}
\end{aligned}
$$

where $\mathfrak{e}$ again denotes a term that obeys $\|\mathfrak{e}\|_{L^{2}} \leq c_{0} r^{-1 / 2}\|\theta\|_{L_{1}^{2}}$.

Step 10 Granted (2-35), then Lemma 2.1 follows from:

Lemma 2.2 Let $v \in \mathbb{R}$ and $\mu \in \mathbb{C}$ denote a given pair of numbers. Fix $m \in\{1,2, \ldots\}$ and use the pair $(\nu, \mu)$ to define the function $h_{:} \mathfrak{C}_{m} \rightarrow \mathbb{R}$ as in (1-6). Fix $\mathfrak{c}=(A, \alpha) \in$ $\mathfrak{C}_{m}$ and define the functions $y$ and $\varsigma$ as in (2-35). Let $\left\{e_{k}=\left(x_{k}, \iota_{k}\right)\right\}_{1 \leq k \leq m}$ denote an orthonormal basis for the $L^{2}$ kernel of the operator $\vartheta_{\mathfrak{c}}$ and thus an orthonormal basis for $\left.T_{1,0} \mathfrak{C}_{m}\right|_{\mathfrak{c}}$. Write a given vector $\left.\zeta \in T_{1,0} \mathfrak{C}_{m}\right|_{\mathfrak{c}}$ as $\zeta=\sum_{1 \leq k \leq m} \zeta^{k} e_{k}$, and write $\left.\left(\nabla_{\zeta_{\mathbb{R}}} \nabla^{1,0} h\right)\right|_{\mathfrak{c}}$ in terms of this basis as $\sum_{1 \leq j \leq m} H^{k} e_{k}$. Then

$$
\begin{aligned}
H^{k}=v \sum_{1 \leq j \leq m} \zeta^{j} \frac{1}{\pi} \int_{\mathbb{C}} \bar{\iota}_{k} \iota_{j}(1 & \left.-\frac{1}{\sqrt{2}} y\right) \\
& +\mu \sum_{1 \leq j \leq m} \zeta^{j} \frac{1}{\pi} \int_{\mathbb{C}}\left(\frac{1}{\sqrt{2}} \bar{x}_{k} \bar{x}_{j}-\frac{1}{2} \varsigma\left(\bar{x}_{k} \bar{\iota}_{j}+\bar{x}_{j} \bar{\iota}_{k}\right)\right) .
\end{aligned}
$$


Proof of Lemma 2.2 Extend the orthonormal basis $\left\{e_{k}=\left(x_{k}, \iota_{k}\right)\right\}_{1 \leq k \leq m}$ for $\left.T_{1,0} \mathfrak{C}_{m}\right|_{\mathfrak{c}}$ to a neighborhood of $\mathfrak{c}$ so as to have vanishing covariant derivative at $\mathfrak{c}$. Fix a smooth function $\mathrm{f}: \mathbb{C} \rightarrow \mathbb{C}$ with $|z|^{R}|\mathrm{f}|$ bounded for some $R \geq 1$. Consider for the moment the section, $\mathfrak{f}$, near $\mathfrak{c}$ of $T_{1,0} \mathfrak{C}_{m}$ whose $e_{k}$ component is given by

$$
\frac{1}{\sqrt{2} \pi} \int_{\mathbb{C}} \mathrm{f} \bar{x}_{k}
$$

The covariant derivative of $\mathfrak{f}$ along $e_{j}$ at $\mathfrak{c}$ has component along $e_{k}$ equal to

$$
\frac{1}{\sqrt{2} \pi} \int_{\mathbb{C}} \partial \mathrm{f} u_{\bar{k} j}
$$

where $u_{\bar{k} j}$ is the $L^{2}$ solution on $\mathbb{C}$ to the equation

$$
-\partial \bar{\partial} u_{\bar{k} j}+\frac{1}{2}|\alpha|^{2} u_{\bar{k} j}=-2^{-1 / 2} \bar{\iota}_{k} \iota_{j}
$$

Meanwhile, the covariant derivative of $\mathfrak{f}$ along $\bar{e}_{j}$ has component along $e_{k}$ equal to

$$
\frac{1}{2 \pi} \int_{\mathbb{C}} \mathrm{f} \alpha \bar{w} \overline{k j}
$$

where $w_{k j}$ is the $L^{2}$ solution on $\mathbb{C}$ to the equation

$$
-\partial_{A} \bar{\partial}_{A} w_{k j}+\frac{1}{2} w_{k j}=-2^{-1 / 2}\left(x_{k} \iota_{j}+x_{j} \iota_{k}\right) .
$$

These formula for the covariant derivative of $\mathfrak{f}$ follow from (2-7) and (2-10) in [14].

Now write $h=h_{v}+h_{\mu}$ where

$$
\begin{aligned}
& \hbar_{v}=\frac{1}{2 \pi} \int_{\mathbb{C}} v|z|^{2}\left(1-|\alpha|^{2}\right), \\
& \hbar_{\mu}=\frac{1}{4 \pi} \int_{\mathbb{C}}\left(\mu \bar{z}^{2}+\bar{\mu} z^{2}\right)\left(1-|\alpha|^{2}\right) .
\end{aligned}
$$

Consider first $\nabla \nabla^{1,0} h_{v}$. To this end, use the top line of (2-13) in [14] to write the $e_{k}$ component of $\nabla^{1,0} h_{v}$ as

$$
-v \frac{1}{\sqrt{2} \pi} \int_{\mathbb{C}} z \bar{x}_{k}
$$

It then follows from (2-37) that the $e_{j}$ covariant derivative of the latter is equal to

$$
-v \frac{1}{\sqrt{2} \pi} \int_{\mathbb{C}} u_{\bar{k} j}
$$


With the preceding understood, note that integration by parts using (2-38) and the left hand equation in (2-34) yields the equality

$$
\int_{\mathbb{C}}\left(1-|\alpha|^{2}\right) u_{\bar{k} j}=\int_{\mathbb{C}} y \bar{\iota}_{k} \iota_{j}
$$

Meanwhile, integrating both sides of (2-38) over $\mathbb{C}$ finds that

$$
\int_{\mathbb{C}}|\alpha|^{2} u_{k j}=-2^{1 / 2} \int_{\mathbb{C}} \bar{\iota}_{k} \iota_{j}
$$

These last two equalities imply that

$$
\frac{1}{\pi} \int_{\mathbb{C}} \bar{\iota}_{k} \iota_{j}\left(1-2^{-1 / 2} y\right)=-\frac{1}{\sqrt{2} \pi} \int_{\mathbb{C}} u_{\bar{k} j}
$$

As explained momentarily, the covariant derivative of $\nabla^{1,0} \xi_{v}$ in the direction of $\bar{e}_{j}$ has $e_{k}$ component equal to zero. Thus, the term proportional to $v$ in the lemma's claim for $H^{k}$ is equal to $\nabla_{\zeta_{\mathbb{R}}}\left(\nabla^{1,0} h_{v}\right)$. To see about the $\bar{e}_{j}$ derivative of $\nabla^{1,0} h_{v}$, use (2-39) to write its $e_{k}$ component as

$$
-v \frac{1}{2 \pi} \int_{\mathbb{C}} z \alpha \bar{w}_{\overline{k j}}
$$

To make something of this equation, multiply both sides of (2-40) by $-v(1 / \pi) \bar{z} \bar{\alpha}$ and integrate the result over $\mathbb{C}$. What with the second line in (1-5), integration by parts finds the left hand side of the resulting equality to be the complex conjugate of the expression in (2-47). Meanwhile, the right hand side of this same equality is equal to the integral over $\mathbb{C}$ of $-v(1 / \pi) \bar{z} \partial\left(x_{k} x_{j}\right)$. This uses the fact that $\vartheta_{\mathfrak{c}} e_{k}=0$ and thus $\partial x_{k}+2^{-1 / 2} \bar{\alpha}_{k}=0$. Thus, an integration by parts finds (2-47) equal to zero.

Consider next $\nabla_{\zeta_{\mathbb{R}}}\left(\nabla^{1,0} h_{\mu}\right)$. The $e_{k}$ component of $\nabla^{1,0} h_{\mu}$ is equal to

$$
-\mu \frac{1}{\sqrt{2} \pi} \int_{\mathbb{C}} \bar{z} \bar{x}_{k}
$$

It follows from (2-37) that the $e_{j}$ covariant derivative of the vector field on $\mathbb{C}$ depicted in (2-43) is zero. Meanwhile, the $\bar{e}_{j}$ derivative of (2-43) can be written as

$$
-\mu \frac{1}{2 \pi} \int_{\mathbb{C}} \bar{z} \alpha \bar{w}_{\overline{k j}}
$$

To proceed from here, note that integration by parts using (2-40) and the right hand equation in (2-34) gives the identity

$$
\frac{1}{\sqrt{2} \pi} \int_{\mathbb{C}} \bar{\zeta}\left(x_{k} \iota_{j}+x_{j} \iota_{k}\right)=\frac{1}{\pi} \int_{\mathbb{C}} \bar{\partial}_{A} \bar{\alpha} w_{k j} .
$$


An integration by parts, and then a second integration by parts with an appeal to the second bullet in (1-5) finds the right hand side of (2-50) equal to

$$
\frac{1}{\pi} \int_{\mathbb{C}} z \bar{\alpha} \partial_{A} \bar{\partial}_{A} w_{k j}
$$

Now use (2-40) to write this last expression as

$$
\frac{1}{\pi} \int_{\mathbb{C}} z \bar{\alpha} w_{k j}+\frac{1}{\sqrt{2} \pi} \int_{\mathbb{C}} z \bar{\alpha}\left(x_{k} \iota_{j}+x_{j} \iota_{k}\right) .
$$

To continue, write $\bar{\alpha} \iota_{k}$ as $-\sqrt{2} \partial x_{k}$ and integrate by parts yet again to see that (2-52) is equal to

$$
\frac{1}{\pi} \int_{\mathbb{C}} z \bar{\alpha} w_{k j}+\frac{1}{\pi} \int_{\mathbb{C}} x_{k} x_{j}
$$

Thus, (2-50) implies that

$$
-\frac{1}{\sqrt{2} \pi} \int_{\mathbb{C}} z \bar{\alpha} w_{k j}=\frac{1}{\pi} \int_{\mathbb{C}}\left(\frac{1}{\sqrt{2}} x_{k} x_{j}-\bar{\varsigma} \frac{1}{2}\left(x_{k} \iota_{j}+x_{j} \iota_{k}\right)\right) .
$$

Thus, the term proportional to $\mu$ in what the lemma asserts is $H^{k}$ is $\nabla_{\zeta_{\mathbb{R}}}\left(\nabla^{1,0} h_{\mu}\right)$.

\section{2.b The relative degree formula: Part I}

Let $\chi_{-}$and $\chi_{+}$denote a pair of elements from $\mathcal{C} \mathcal{Z}^{*}$. The previous section proves that the large $r$ versions of both $\mathfrak{c}_{-}=\Phi^{r}\left(\chi_{-}\right)$and $\mathfrak{c}_{+}=\Phi^{r}\left(\chi_{+}\right)$are nondegenerate solutions to (1-8). This is to say that the $\mathfrak{c}=\mathfrak{c}_{ \pm}$versions of the operator $\mathfrak{L}_{\mathfrak{c}}$ in (1-8) have trivial kernel. The subsection and the next two prove the formula that Theorem 1.1 asserts for the difference $\operatorname{deg}_{\mathrm{SW}}\left(\mathfrak{c}_{+}\right)-\operatorname{deg}_{\mathrm{SW}}\left(\mathfrak{c}_{-}\right)$. Assume here and in the next subsection that both $\Theta_{-}$and $\Theta_{+}$are such that if $(\gamma, m)$ is in either of these sets, then $\gamma$ has a tubular neighborhood map $\varphi: S^{1} \times D \rightarrow M$ with the following properties:

- If $\gamma$ is hyperbolic, then $m=1$. Furthermore, $(\nu, \mu)=\left(\frac{1}{4} k, i \varepsilon e^{i k t}\right)$ with $k \in \mathbb{Z}$ and with $\varepsilon>0$ but very small.

- If $\gamma$ is elliptic, then its rotation number $\mathrm{R}$ is irrational. Furthermore,

(i) The pair $(\nu, \mu)=\left(\frac{1}{2} \mathrm{R}, 0\right)$.

(ii) The $\varphi^{*}$-pullback of $T^{1,0}(\mathbb{R} \times M)$ is spanned by $d s+i a$ and $\left(\ell_{\gamma} /(2 \pi)\right)(d z-i \mathrm{R} z d t)$.

It is a consequence of Lemmas 2.1-2.4 in [14] that $\chi_{-}$and $\chi_{+}$are as follows: If $(\gamma, m)$ is in either $\Theta_{-}$or $\Theta_{+}$, then the associated map from $S^{1}$ to $\mathfrak{C}_{m}$ is the constant map to the symmetric vortex; this the solution to (1-5) with $\alpha^{-1}(0)=0 \in \mathbb{C}$. 
Much of the notation that appears in these sections comes from Sections 4-7 of [14]. The discussion in this subsection has eight parts.

Part 1 Fix a smooth, oriented, embedded surface $\Sigma \subset \mathbb{R} \times M$ with no compact components whose projection to $M$ defines a homology between the cycles $\Sigma_{(\gamma, m) \in \Theta_{-}} m \gamma$ and $\Sigma_{(\gamma, m) \in \Theta_{+}} m \gamma$. To be explicit, fix $R \gg 1$ as in (4-8) of [14] and require that the $|s| \geq R$ portion of $\Sigma$ be a disjoint union of embedded cylinders. Each such cylinder is deemed an end of $\Sigma$. These ends behave as follows: Let $(\gamma, m) \in \Theta_{-}$. Then there are precisely $m$ ends of $\Sigma$ where $s \ll-1$ whose constant $s$ slices converge as $s \rightarrow-\infty$ to $\gamma$ as a degree 1 braid in a tubular neighborhood of $\gamma$. Such an end, $\mathcal{E}$, appears as follows: Extend the coordinate map $\varphi$ in (2-55) to give coordinates $(w, t, z) \in \mathbb{R} \times S^{1} \times D$ to a neighborhood of $\mathbb{R} \times \gamma$ in $\mathbb{R} \times M$ via the map that sends any given point $(w, t, z)$ to the point $\left(s=w-\frac{1}{2}|z|^{2}, \phi(t, z)\right)$. The end $\mathcal{E}$ appears in these coordinates as the graph of the map from $(-\infty,-R] \times S^{1}$ to $D$ that sends $(w, t)$ to $z=z_{\mathbb{E}} \zeta(t) e^{-2 \lambda w}$. Here $\lambda$ is the largest of the negative eigenvalue of $\gamma$ 's version of (1-2) on $S^{1}=\mathbb{R} / 2 \pi \mathbb{Z}$ and $\varsigma(t)$ is the corresponding eigenvector. Meanwhile $z_{\mathcal{E}} \in \mathbb{C}-0$ is an $m$-th root of unity, chosen so that $z_{\mathcal{E}} \neq z_{\mathcal{E}^{\prime}}$ when $\mathcal{E}$ and $\mathcal{E}^{\prime}$ are distinct ends whose constant s slices limit to $\gamma$. Each $s \gg 1$ end of $\Sigma$ has an analogous description but with $\lambda$ the smallest positive eigenvalue.

With $\Sigma$ fixed, introduce $U_{\Sigma} \rightarrow \mathbb{R} \times M$ to denote a tubular neighborhood of $\Sigma$ 's intersection with $[-4 R, 4 R]$. If $(\gamma, m) \in \Theta_{-}$, let $U_{\gamma^{-}}$denote the set of points in $\gamma$ 's version of $\mathbb{R} \times S^{1} \times D$ with $w<-2 R$ and distance less than $4 \rho_{*}$ from $\gamma$. Here, $\rho_{*}>0$ is the constant that appears in (4-8) of [14]. If $(\gamma, m) \in \Theta_{+}$, use $U_{\gamma+}$ to denote the set of points in $\gamma$ 's version of $\mathbb{R} \times S^{1} \times D$ with $w>2 R$ and distance less than $4 \rho_{*}$ from $\gamma$.

Part 2 Fix an orthogonal, almost complex structure on a neighborhood of $\Sigma$ in $\mathbb{R}$ so that $\Sigma$ is pseudoholomorphic. Denote this almost complex structure as $J_{\Sigma}$. Given the description above for the ends of $\Sigma$, this almost complex structure can be chosen so that it differs little from the already chosen almost complex structure $J$ where $|s|$ is large. In particular it can be assumed that $\left|J-J_{\Sigma}\right| \leq c_{0} e^{-|s| / c_{0}}$.

Let $\pi: N \rightarrow \Sigma$ denote the normal bundle to $\Sigma$. The almost complex structure $J_{\Sigma}$ gives $N$ the structure of a complex line bundle over $\Sigma$. Use $\theta$ in what follows to denote both the Hermitian connection on $N$ and also its pullback to $\pi^{*} N \rightarrow N$. Also, use $\mathfrak{s}$ to denote the tautological section of $\pi^{*} N$. With $R$ fixed, let $\Sigma_{R}$ denote the part of $\Sigma$ where $|s| \leq 4 R$. The metric's exponential map embeds a fixed radius subbundle of $\left.N\right|_{\Sigma_{R}}$. The latter bundle is denoted in what follows as $N_{1}$. This exponential map is used implicitly in what follows to identify $N_{1}$ with its image in $\mathbb{R} \times M$. 
Because $J_{\Sigma}$ is orthogonal, and because $\Sigma$ is $J_{\Sigma}$ pseudoholomorphic, there is a self dual section, $\omega_{\Sigma}$, of $\bigwedge^{2} T^{*}(\mathbb{R} \times M)$ over $((-\infty,-R] \times M) \cup([R, \infty) \times M) \cup U_{\Sigma}$ whose pullback to $\Sigma$ is the induced area form and whose $|s| \rightarrow \infty$ limit in either direction is the form $\omega_{a}=d s \wedge a+* a$. Given the convergence of $J_{\Sigma}$ to $J$ at large $|s|$, no generality is lost by requiring that the limit is approached exponentially fast:

$$
\lim _{|s| \rightarrow \infty}\left|\omega_{\Sigma}-\omega_{a}\right| \leq c_{0} e^{-|s| / c_{0}}
$$

This form $\omega_{\Sigma}$ can be chosen so that it appears on $N_{1}$ as

$$
\omega_{\Sigma}=\frac{i}{2} \nabla_{\theta} \mathfrak{s} \wedge \nabla_{\theta} \overline{\mathfrak{s}}+\pi^{*} \operatorname{vol}_{\Sigma}+\mathcal{O}\left(|\mathfrak{s}|^{2}\right)
$$

where $\mathfrak{s :} N_{1} \rightarrow \pi^{*} N$ is the tautological section, and where $\operatorname{vol}_{\Sigma}$ denotes the induced area 2 -form on $\Sigma$.

Extend $\omega_{\Sigma}$ as a self-dual 2 -form to the whole of $\mathbb{R} \times M$ with transversal zero locus, $Z \subset(-1,1) \times M$. The form $\omega_{\Sigma}$ can be modified if necessary so as to insure that $\mathcal{Z}$ is either empty or has two connected components.

To elaborate, there are coordinates near any give component of $z$ which are such that $\omega_{\Sigma}$ appears as

$$
\omega_{\Sigma}=d t \wedge u_{i j} x^{i} d x^{j}+\frac{1}{2} u_{i j} x^{i} \varepsilon^{j k n} d x^{k} d x^{n}+\mathcal{O}\left(|x|^{2}\right),
$$

where the notation is as follows: The coordinates are on $S^{1} \times B^{3}$ where $B^{3} \subset \mathbb{R}^{3}$ is a ball centered on the origin; $t \in \mathbb{R} /(2 \pi \mathbb{Z})$ is an affine coordinate on $S^{1}$ and $\left(x^{1}, x^{2}, x^{3}\right)$ are the Euclidean coordinates on $\mathbb{R}^{3}$. What are denoted by $\left\{u_{i j}\right\}_{1 \leq i, j \leq 3}$ are the entries of a smooth map $t \rightarrow u(t)$ from $S^{1}$ to $\operatorname{SL}(3 ; \mathbb{R})$. Meanwhile, $\varepsilon^{i j k}$ is the completely antisymmetric 3 -tensor with $\varepsilon^{123}=1$. Finally, the summation convention for repeated indices are used. The map $u$ may or may not be homotopically trivial. If it is, there is a further change of coordinates that makes $u$ to be the constant map to the identity matrix. It follows from an observation of Gompf [4] that $\omega_{\Sigma}$ can be chosen so that each component of $z$ has a version of $u$ that is homotopically trivial. This extra requirement can be met only if $z$ has even number of components, and two components is always sufficient. Assume such a version of $\omega_{\Sigma}$ and assume that the coordinates in (2-58) are such that $u$ is the constant map to the identity element.

One more constraint on $Z$ is required: Assume that the projection from $\mathbb{R} \times M$ to $M$ sends $Z$ to the complement of $\bigcup_{(\gamma, m) \in \Theta_{-}} \gamma$ and $\bigcup_{(\gamma, m) \in \Theta_{+}} \gamma^{\prime}$. 
Part 3 Let $T \subset(-1,1) \times M$ denote a tubular neighborhood of $Z$ that is disjoint from $\Sigma$. Use $X_{Z}$ to denote $(\mathbb{R} \times M)-Z$. Modify the Riemannian metric on $\mathbb{R} \times M$ so that the resulting metric has the following properties: First, this metric should agree with the original on $(\mathbb{R} \times M)-T$. Second, there is a smaller tubular neighborhood $T^{\prime} \subset T$ of each component of $Z$ such that $T^{\prime}-\left(T^{\prime} \cap Z\right) \subset X_{Z}$ is isometric to the product $\left(D^{2}-0\right) \times S^{2}$ where $D^{2} \subset \mathbb{C}$ is the disk of radius 1 centered at the origin with the Euclidean metric. Let $X$ denote the Riemannian manifold that is obtained by first deleting each version of $T^{\prime}$ from $\mathbb{R} \times M$ and then attaching $D^{2} \times S^{2}$ using the aforementioned isometry to identify the complement in $T^{\prime}$ of $T^{\prime} \cap Z$ with $\left(D^{2}-0\right) \times S^{2}$.

The form $\omega_{\Sigma}$ can be chosen so as to have a modification on $T$ that extends to the whole of $X$ as a self-dual form, this denoted by $\omega_{X}$. This form $\omega_{X}$ has the following properties: It agrees with $\omega_{\Sigma}$ on $X-T=(\mathbb{R} \times M)-T$; it is self-dual with respect to the metric on $X$; and it appears on each $(D-0) \times S^{2} \subset T-Z$ as $\omega_{X}=\omega_{D}+\omega_{S^{2}}$ where $\omega_{D}$ is the area form on the disk $D$ and $\omega_{S^{2}}$ is the area form on $S^{2}$ with the latter viewed as the unit sphere in $\mathbb{R}^{3}$.

Part 4 An oriented, Riemannian 4-manifold with a $\operatorname{Spin}_{\mathbb{C}}$-structure has the $\mathbb{C}^{2}$ bundles of self-dual and anti self-dual spinors. These are associated to the given $\operatorname{Spin}_{\mathbb{C}}(4)=\left(\operatorname{SU}(2) \times \operatorname{SU}(2) \times S^{1}\right) /\{ \pm 1\}$ lift of the principal $\mathrm{SO}(4)$ frame bundle of the tangent bundle. They are obtained by composing the defining representation of $U(2)$ on $\mathbb{C}^{2}$ with the two evident homomorphisms of $\operatorname{Spin}_{\mathbb{C}}(4)$ to $U(2)=\left(\operatorname{SU}(2) \times S^{1}\right) /\{ \pm\}$. These two spinor bundles are described at the beginning of Chapter 1 of [9]. When $M$ is a 3-dimensional Riemannian manifold, a $\operatorname{Spin}_{\mathbb{C}}$ structure on $M$ induces one on $\mathbb{R} \times M$. If the latter has its product metric, then the $\mathrm{SO}(4)$ frame bundle of $\mathbb{R} \times M$ is isomorphic to the pullback from $M$ of the $\mathrm{SO}(4)$ bundle obtained from the $\mathrm{SO}(3)$ principal frame bundle of $M$ via the standard inclusion homomorphism of $\mathrm{SO}(3)$ in $\mathrm{SO}(4)$. The latter isomorphism induces a canonical isomorphism from the self-dual spinor bundle on $\mathbb{R} \times M$, and also one from the anti self-dual spinor bundle, to the pullback of the spinor bundle over $M$.

Granted the preceding, view the spinor bundle $\mathbb{S}_{I}$ as the bundle of self-dual spinors on the 4-manifold $\mathbb{R} \times M$. By restriction, the associated $\operatorname{Spin}_{\mathbb{C}}$ structure defines an analogous bundle of self-dual spinors on $X_{Z}$. The latter is denoted by $\mathbb{S}_{I, X}$. Clifford multiplication by $\omega_{X}$ defines a splitting of $\mathbb{S}_{I, X}$ on $X_{Z}$ as a direct sum of two complex line bundles. This splitting is written as

$$
\mathbb{S}_{I, X}=L \oplus\left(L K_{X}^{-1}\right),
$$

where the convention takes the left most summand to be the $+i\left|\omega_{X}\right|$ eigenspace of Clifford multiplication by $\omega_{X}$ and the right most to be the $-i\left|\omega_{X}\right|$ eigenspace. Note 
that this splitting is very close where $|s| \geq R$ to the splitting that is defined by Clifford multiplication by the 1 -form $a$.

Consider now the line bundles $K_{X} \rightarrow X_{Z}$ and $L \rightarrow X_{Z}$. By definition, $L$ restricts to the $|s| \geq 1$ part of $X$ as the trivial bundle and $K_{X}$ restricts as the bundle $K$. To say more, note that the bundle $K_{X}$ restricts to any $\left(D^{2}-0\right) \times S^{2}$ end in $X$ as the pullback from $S^{2}$ of the bundle whose first Chern class is twice the generator of $H^{2}\left(S^{2} ; \mathbb{Z}\right)$. Meanwhile, $L$ restricts to this part of $X$ as the pullback from $S^{2}$ of the bundle whose first Chern class generates $H^{2}\left(S^{2} ; \mathbb{Z}\right)$. Indeed, this can be seen using (2-58) given that $\mathbb{S}_{I}$ extends over $Z$. This analysis leads to the conclusion that $K_{X}=L^{2} K$.

It follows from what was just said that $\mathbb{S}_{I, X}$ extends from $X_{Z}$ to $X$ as the bundle of self-dual spinors for a $\operatorname{Spin}_{\mathbb{C}}$ bundle on $X$. Use $\mathbb{S}_{I+}$ to denote the bundle $L^{-1} \otimes \mathbb{S}_{I, X}$. This splits as $I_{\mathbb{C}} \oplus K_{X}{ }^{-1}$ with respect to Clifford multiplication by $\omega_{X}$. Use $\mathbb{S}_{E+} \rightarrow X$ in what follows to denote $\left(E L^{-1}\right) \otimes \mathbb{S}_{I, X}=E \oplus E K_{X}{ }^{-1}$. Both $\mathbb{S}_{I+}$ and $\mathbb{S}_{E+}$ are spinor bundles for $\operatorname{Spin}_{\mathbb{C}}$ structures on $X$.

Part 5 As noted previously, the bundle $L$ restricts to $\{ \pm 1\} \times M$ as the trivial bundle. Moreover, it has a canonical trivialization here using the section $1_{\mathbb{C}}$. With this trivialization understood, it's first Chern class has a well defined pairing with $\Sigma$. This pairing is denoted in what follows by $k_{\Sigma}$.

To say more about $k_{\Sigma}$, let $\chi^{\Sigma}$ denote the Euler characteristic of $\Sigma$. Meanwhile, let $\operatorname{deg}\left(N_{\Sigma}\right)$ denote the Euler class of the normal bundle of $\Sigma$ as defined using the section whose restriction to any given end is the real part of $\partial / \partial z$. Let $-\left\langle c_{1}, \Sigma\right\rangle$ denote the Euler class of the restriction in $X$ of $K$ to $\Sigma$ as defined using a section whose restriction to any given end is also the real part of $\partial / \partial z$.

Lemma 2.3 The integer $k_{\Sigma}$ is equal to $-\frac{1}{2}\left(\left\langle c_{1}, \Sigma\right\rangle+\chi^{\Sigma}+\operatorname{deg}\left(N_{\Sigma}\right)\right)$.

Proof of Lemma 2.3 A section of $L$ is defined as follows: Let $\psi_{0}$ denote a unit length section of $\mathbb{S}_{I}$ with the property $\operatorname{cl}(a) \psi_{0}=i \psi_{0}$. Then $\left(\operatorname{cl}\left(\omega_{X}\right)+i \sqrt{2}\left|\omega_{X}\right|\right) \psi_{0}$ defines a section of $L$. The latter vanishes on $\Sigma$ at those points where $\omega_{X}=-\left(d s \wedge a+\frac{1}{2} d a\right)$. To see what this means, fix a trivialization of $T^{*} M$. This then induces a trivialization of the $\mathbb{R}^{3}$ bundle of self-dual forms on $\mathbb{R} \times M$, and thus its restriction to $\Sigma$. Both $\left(d s \wedge a+\frac{1}{2} d a\right)$ and $\omega_{X}$ are nowhere zero, self-dual 2-forms along $\Sigma$. With this bundle trivialized, both define a map from $\Sigma$ to $S^{2}$ and together they define a map from $\Sigma$ to $S^{2} \times S^{2}$ with limit on the diagonal as $|S| \rightarrow \infty$. This understood, the image of $\Sigma$ via this map has a well defined intersection number with the antidiagonal in $S^{2} \times S^{2}$. This intersection number is $k_{\Sigma}$. 
To write this intersection number as in the statement of the lemma, again view $\omega_{X}$ as a map to $S^{2}$, and consider its pullback of the tangent bundle of $S^{2}$. A section of $\omega_{X}^{*} T S^{2}$ is a self-dual 2 -form along $\Sigma$ that is orthogonal to $\omega_{X}$. Fix such a form with the following property: On any given end in any $(\gamma, m) \in \Theta_{-}$version of $U_{\gamma-}$ or $(\gamma, m) \in \Theta_{+}$version of $U_{\gamma+}$, require that the $|w| \rightarrow \infty$ limit equal the real part of $(d w+i d t) \wedge d z$. Use $\mathfrak{u}$ to denote such a self-dual 2 -form. Then $\mathfrak{u}\left(b, b^{\prime}\right)=0$ if $b$ and $b^{\prime}$ are sections of $T \Sigma$. Thus $\mathfrak{u}$ defines a homomorphism from $T \Sigma$ to the dual of the normal bundle $N \rightarrow \Sigma$. This homomorphism on any given end of $\Sigma$ maps the vector field $\partial / \partial s$ to the real part of $d z$. It follows as a consequence that the degree of $\mathfrak{u}$ as a section of $\left.\omega_{X} * T S^{2}\right|_{\Sigma}$ is $-\left(\chi^{\Sigma}+\operatorname{deg}\left(N_{\Sigma}\right)\right)$ where $\operatorname{deg}\left(N_{\Sigma}\right)$ denotes the euler class of the normal bundle of $\Sigma$ as defined using any section whose restriction to any given end is the real part of $\partial / \partial z$. Consider next the pullback via the map $\left(d s \wedge a+\frac{1}{2} d a\right)$ of the tangent bundle to $S^{2}$. A section of the latter bundle is, by definition, a section of the bundle $K$. Fix a section whose restriction at large $|s|$ at any given end is the real part of $d z(\partial / \partial z)$. The Euler class of this section is well defined and is equal to $\left\langle c_{1}, K\right\rangle$. The claim about $k_{\Sigma}$ follows directly from these observations.

Part 6 Follow the instructions given in Section 5.a of [14] to construct a pair $\left(A_{X}^{*}, \psi_{X}^{*}\right)$ of section of $\operatorname{Conn}(E)$ over $X$ and section of $\mathbb{S}_{E+}$ over $X$. Note in this regard that the spinor $\psi_{X}^{*}$ appears as $\left(\alpha_{X}, 0\right)$ with respect to the splitting of $\mathbb{S}_{E+}$ that is defined by Clifford multiplication of $\omega_{X}$. Here, $\alpha_{X}$ is a section of $E$ that is $A_{X}^{*}$-covariantly constant on the complement of a neighborhood of $\Sigma$. In particular, $A_{X}^{*}$ has zero curvature and zero holonomy on the $[0, \infty) \times S^{1} \times S^{2}$ part of $X$.

Each pair $(\gamma, m) \in \Theta_{-}$also has its $\xi=0$ version of what is denoted as $\mathfrak{p}_{\xi}$ in Part 5 of the proof of Lemma 6.3 in [14]. Introduce the corresponding $\mathfrak{b}_{\gamma-}=\left(0, \mathfrak{p}_{\xi=0}\right)$, this defined a priori as a map from $(-\infty,-R) \times S^{1} \times \mathbb{C}$ to $\mathbb{V}_{0} \oplus \mathbb{V}_{1}$. Define the set $U_{\gamma-} \subset \mathbb{R} \times M$ as in (5-3) of [14] and view $\mathfrak{b}_{\gamma-}$ on the set $U_{\gamma-}$ as a section of $i T^{*} M \oplus \mathbb{S} \oplus i \mathbb{R}$. There is an analogous $\mathfrak{b}_{\gamma+}$ for each pair $(\gamma, m) \in \Theta_{+}$.

Let $\mathcal{C}$ denote the set of components of $\Sigma$. Define the tubular neighborhood $U_{C}$ for each $C \in \mathcal{C}$ as in (5-3) of [14]. Each $C \in \mathcal{C}$ has a corresponding section $\mathfrak{b}_{C}$ as defined Part 3 of the proof of Lemma 6.3 in [14]. This is viewed as a section over the set $U_{C}$ of $i T^{*} M \oplus \mathbb{S} \oplus i \mathbb{R}$.

Granted the preceding definitions, introduce

$$
\begin{aligned}
\mathfrak{h}=\sum_{C \in \mathcal{C}}\left(\chi_{C} \prod_{\mathcal{E} \in \mathcal{E}_{C}}\left(1-\chi_{\mathcal{E}_{C}}\right) \mathfrak{b}_{C}\right)+ & \sum_{\mathcal{E} \in \mathcal{E}_{C}} \sum_{(\gamma, m) \in \Theta} \chi_{\mathcal{E}_{C}} \chi_{\gamma}-\mathfrak{b}_{\gamma-} \\
& +\sum_{\mathcal{E} \in \mathcal{E}_{C}} \sum_{(\gamma, m) \in \Theta^{\prime}} \chi_{\mathcal{E}_{C}} \chi_{\gamma+} \mathfrak{b}_{\gamma+}
\end{aligned}
$$


where $\mathcal{E}_{C}$ denotes the set of ends of $C$ and where $\chi_{\mathcal{E}_{C}}$ is defined as follows: Let $C \in \mathcal{C}$ denote the component that contains $\mathcal{E}$. Introduce from Part 2 of Section 4.a in [14] the normal bundle to $C$ with its exponential map, $e_{C}$, onto a tubular neighborhood of $C$. Introduce from Step 1 of Part 1 in Section 5.a of [14] the disk subbundle $N_{4 C} \rightarrow C$ in $C$ 's normal bundle. Finally, introduce the constants $R, \rho_{\Sigma}$ and $R_{\mathcal{E}}$ from (4-8) in [14]. What is denoted by $\chi_{\mathcal{E}_{C}}$ above is the function on $\mathbb{R} \times M$ with compact support on $e_{C}\left(\left.N_{4 C}\right|_{\mathcal{E}}\right)$ where it is given by the rule $\chi_{\mathcal{E}_{C}}=\chi\left(4|\mathfrak{s}| / \rho_{\Sigma}\right) \chi\left(\left(R_{\mathcal{E}}+3 R-|w|\right) / R\right)$.

View $\mathfrak{h}$ as a section of $i T^{*} X \oplus \mathbb{S}_{E}$ over the whole of $X$ which is nonzero only on the part of $X$ that is isometric to $\mathbb{R} \times M$, thus where $T^{*} X$ splits as $T^{*} M \oplus \mathbb{R}$. Write $\mathfrak{h}=(c, \varsigma)$. Define $A_{X}$ to be the connection on $E$ over $X$ given by $A_{X}^{*}+\sqrt{2} r^{1 / 2} c$. Meanwhile, define $\psi_{X}$ to be the section of $\mathbb{S}_{E+}$ over $X$ given by $\psi_{X}^{*}+\varsigma$.

Part 7 Let $\mathbb{S}_{E-}$ denote the bundle of anti-self-dual spinors on $X$ that is defined by the $\operatorname{Spin}_{\mathbb{C}}$ structure with self-dual spinor bundle $\mathbb{S}_{E+}$. Let $\Lambda^{2+} \rightarrow X$ denote the bundle of self-dual 2-forms on $X$. These bundles appear implicitly in the upcoming Equation (2-61) which describes an operator $\mathfrak{D}_{E}: C^{\infty}\left(X ; i T^{*} X \oplus \mathbb{S}_{E+}\right) \rightarrow$ $C^{\infty}\left(X ; i \Lambda^{2+} \oplus \mathbb{S}_{E-} \oplus i \mathbb{R}\right)$ that plays a central role in the subsequent parts of the proof.

To start the definition of $\mathfrak{D}_{E}$, fix a Hermitian connection on $K_{X}$ with the following properties: First, it restricts to the $|s| \geq 1$ part of $X$ as the connection $A_{K}$ on $K$ that is described in Part 3 of Section 1.a. Second, it restricts to each end of $X-\Sigma$ as the pullback of a connection on the $S^{2}$ factor of $[0, \infty) \times S^{1} \times S^{2}$. Use $\Delta_{A_{X}}: C^{\infty}\left(X ; \mathbb{S}_{E+}\right) \rightarrow C^{\infty}\left(X ; \mathbb{S}_{E-}\right)$ to denote the Dirac operator that is defined by this connection on $K_{X}$ and the connection $A_{X}$ on $E$. Use $d^{+}: C^{\infty}\left(X ; i T^{*} X\right) \rightarrow$ $C^{\infty}\left(X ; \Lambda^{2+}\right)$ to denote the self-dual projection of the exterior derivative. Meanwhile, use $d^{*}: C^{\infty}\left(X ; i T^{*} X\right) \rightarrow C^{\infty}(X ; i \mathbb{R})$ to denote the formal $L^{2}$ adjoint of the exterior derivative. The operator $\mathfrak{D}_{E}$ sends any given section $(6, \eta)$ of $i T^{*} X \oplus \mathbb{S}_{E+}$ to the section of the bundle $i \Lambda^{2+} \oplus \mathbb{S}_{E-} \oplus i \mathbb{R}$ whose respective components are

- $d^{+} 6-2^{-1 / 2} r^{1 / 2}\left(\psi_{X}^{\dagger} \tau \eta+\eta^{\dagger} \tau \psi_{X}\right)$,

- $\Delta_{A_{X}} \eta+2^{1 / 2} r^{1 / 2} \operatorname{cl}(6) \psi_{X}$,

- $d^{*} b-2^{-1 / 2} r^{1 / 2}\left(\eta^{\dagger} \psi_{X}-\psi_{X}^{\dagger} \eta\right)$.

Lemma 2.4 There exists $\kappa>1$ with the following significance: If $r \geq \kappa$, then the operator $\mathfrak{D}_{E}$ defines a Fredholm operator from $L_{1}^{2}\left(X ; i T^{*} X \oplus \mathbb{S}_{E+}\right)$ to the Hilbert space $L^{2}\left(X ; i \Lambda^{2+} \oplus \mathbb{S}_{E-} \oplus i \mathbb{R}\right)$.

Proof of Lemma 2.4 The assertion follows from the fact that both the $\mathfrak{c}_{-}=\Phi^{r}\left(\chi_{-}\right)$ and $\mathfrak{c}_{+}=\Phi^{r}\left(\chi_{+}\right)$versions of (1-9) have trivial kernel. 
Part 8 The following lemma motivates the interest in $\mathfrak{D}_{E}$. The lemma uses $\operatorname{ind}\left(\mathfrak{D}_{E}\right)$ to denote its index as a Fredholm operator with to the domain and range spaces that are described in Lemma 2.4.

Lemma 2.5 The spectral flow from $\mathfrak{L}_{\mathfrak{c}_{-}}$to $\mathfrak{L}_{\mathfrak{c}_{+}}$is equal to ind $\left(\mathfrak{D}_{E}\right)+2 k_{\Sigma}$.

Proof of Lemma 2.5 The index of $\mathfrak{D}_{E}$ is insensitive to any change of the connections and spinors used to define this operator as long as such changes have compact support. Keep this fact in mind in what follows.

Reintroduce the pair $\left(A_{-}, \psi_{-}\right)=\left(A^{*}+2^{1 / 2} r^{1 / 2} b_{*}, \psi^{*}+\eta_{*}\right)$ as defined in Step 2 of Section 2.a using the data from the set $\Theta_{-}$. Let $\left(A_{+}, \psi_{+}\right)$denote the corresponding $\Theta_{+}$version. Fix $R^{\prime} \gg R$ and deform the pair $\left(A_{X}, \psi_{X}\right)$ to $\left(A_{X}^{\prime}, \psi_{X}^{\prime}\right)$ with the following properties: This pair is equal to $\left(A_{X}, \psi_{X}\right)$ where $s \leq 1$. Where $s \in\left(2, R^{\prime}\right)$, it is equal to $\left(A_{-}, \psi_{-}\right)$. Finally, it is equal to $\left(A_{+}, \psi_{+}\right)$where $s \geq 2 R^{\prime}$. Let $\mathfrak{D}_{E}^{\prime}$ denote the $\left(A_{X}^{\prime}, \psi_{X}^{\prime}\right)$ version of (2-61). The operators $\mathfrak{D}_{E}$ and $\mathfrak{D}_{E}^{\prime}$ have the same large $|s|$ limits and so $\mathfrak{D}_{E}^{\prime}$ is also Fredholm as a linear map from $L_{1}^{2}\left(X ; i T^{*} X \oplus \mathbb{S}_{E+}\right)$ to $L^{2}\left(X ; i \Lambda^{2+} \oplus \mathbb{S}_{E-} \oplus i \mathbb{R}\right)$. Moreover, its index is the same as that of $\mathfrak{D}_{E}$.

Granted the preceding, let $\mathfrak{D}_{E}^{\prime \prime}$ denote the operator from $L_{1}^{2}\left(X ; i T^{*} X \oplus \mathbb{S}_{E+}\right)$ to $L^{2}\left(X ; i \Lambda^{2+} \oplus \mathbb{S}_{E-} \oplus i \mathbb{R}\right)$ that is defined via (2-61) using $\left(A^{\prime \prime}, \psi^{\prime \prime}\right)$ where the latter is declared equal to $\left(A_{X}^{\prime}, \psi_{X}^{\prime}\right)$ where $s \leq 2$ and equal to $\left(A_{-}, \psi_{-}\right)$where $s \geq 2$. This operator is also Fredholm. Take $R^{\prime}$ large and a standard Mayer-Vietoris argument can be used to prove that index $\left(\mathfrak{D}_{E}^{\prime}\right)=\operatorname{index}\left(\mathfrak{D}_{E}^{\prime \prime}\right)+f\left(\mathfrak{c}_{-}, \mathfrak{c}_{+}\right)$, where $f$ denotes the spectral flow from $\mathfrak{L}_{\mathfrak{c}_{-}}$to $\mathfrak{L}_{\mathfrak{c}_{+}}$.

Meanwhile, the operator $\mathfrak{D}_{E}^{\prime \prime}$ where $s \leq-R$ is the same as where $s \geq R$. As a consequence, its index is that of an operator on the compact manifold, $Y$, that is obtained by identifying $\{-R\} \times M \subset X$ with $\{+R\} \times M \subset X$. This understood, the index of $\mathfrak{D}_{E}^{\prime \prime}$ can be computed using the Atiyah-Singer Index Theorem. This theorem finds ind $\left(\mathfrak{D}_{E}^{\prime \prime}\right)=-2 k_{\Sigma}$. Thus, $\operatorname{ind}\left(\mathfrak{D}_{E}\right)=-2 k_{\Sigma}+f\left(\mathfrak{c}_{-}, \mathfrak{c}_{+}\right)$. This is what is stated by the lemma.

\section{2.c The relative degree formula: Part II}

This subsection computes the index of $\mathfrak{D}_{E}$ and completes the proof of the degree formula in Theorem 1.1 for the case that (2-55) holds for each Reeb orbit $\gamma$ from either $\Theta_{-}$or $\Theta_{+}$. Note that (2-55) is assumed in this subsection. The discussion here has eight parts. 
Part 1 This part of the proof begins the task of computing ind $\left(\mathfrak{D}_{E}\right)$. To set notation, let $\nabla$ denote the covariant derivative on both $i T^{*} X \oplus \mathbb{S}_{E+}$ and $i \Lambda^{2+} \oplus \mathbb{S}_{E-} \oplus i \mathbb{R}$ as defined using the Riemannian connection from $T^{*} X$, the connection chosen for $K_{X}$ and the connection $A_{X}$ for $E$. Define the Hilbert space $\mathbb{H}_{X}$ to denote the space of $L_{1}^{2}$ sections of $i T^{*} X \oplus \mathbb{S}_{E+}$ with the norm

$$
\|\mathfrak{q}\|_{\mathbb{H}, X^{2}}=\int_{X}|\nabla \mathfrak{q}|^{2}+\frac{1}{4} r \int_{X}|\mathfrak{q}|^{2}
$$

Meanwhile, use $\mathbb{L}_{X}$ to denote the Hilbert space $L^{2}\left(X ; i \Lambda^{2+} \oplus \mathbb{S}_{E-} \oplus i \mathbb{R}\right)$.

Define the Hilbert space $\mathcal{K}_{1}^{2}$ as in (5-12)-(5-13) of [14] using the $L_{1}^{2}$ norm rather than the $\mathcal{K}$-norm. Define the map $t_{X}: \mathcal{K}_{1}^{2} \rightarrow \mathbb{H}_{X}$ by copying the definition in (6-9) of [14] for its map $t$. Introduce $\Pi_{X}: \mathbb{H}_{X} \rightarrow \mathbb{H}_{X}$ to denote the $L^{2}$ orthogonal projection onto the image of $t$. Use $\mathbb{H}_{X}^{\perp}$ to denote $\left(1-\Pi_{X}\right) \mathbb{H}_{X}$. Note that $t_{X}$ is a bounded injection when $r \geq c_{0}$ and $R \geq c_{0}$. Mimic what is done in Part 7 of Section 6.a in [14] to define the space $\mathcal{L}^{2}$. The analogous version of $t_{X}$ maps $\mathcal{L}^{2}$ injectively into $\mathbb{L}_{X}$. Use $\Pi_{X}$ also to denote the orthogonal projection on $\mathbb{L}_{X}$ with image $t_{X}\left(\mathcal{L}^{2}\right)$. Finally, use $\mathbb{L}_{X}^{\perp}$ to denote $\left(1-\Pi_{X}\right) \mathbb{L}_{X}$. Note that when $r \geq c_{0}$ and $R \geq c_{0}$, the map $t_{X}: \mathcal{L}^{2} \rightarrow \mathbb{L}_{X}$ is nearly isometric because

$$
\left(1-c_{0} r^{-\sigma / 4}\right)\|\theta\|_{\mathcal{L}^{2}} \leq\left\|t_{X}(\theta)\right\|_{L^{2}} \leq\left(1+c_{0} r^{-\sigma / 4}\right)\|\theta\|_{\mathcal{L}^{2}}
$$

Here, and below $\|\cdot\|_{\mathcal{L}^{2}}$ denotes the norm on $\mathcal{L}^{2}$. Meanwhile, $\sigma$ is the positive constant that is introduced in Section 4.c of [14]. By way of explanation, these inequalities follow because the various identifications of the bundle $i T^{*} X \oplus \mathbb{S}_{E+}$ that are used to define $t_{X}$ are nearly isometric. See Part 6 of Section 6.a in [14] and (5-12)-(5-13) in [14].

The operator $\mathfrak{D}_{E}$ is analyzed below by decomposing $\mathbb{H}_{X}$ as $\mathbb{H}_{X}^{\perp} \oplus t_{X}\left(\mathcal{K}_{1}^{2}\right)$ and $\mathbb{L}_{X}$ as $\mathbb{L}^{\perp} \oplus t_{X}\left(\mathcal{L}^{2}\right)$. To this end, write $\mathfrak{f} \in \mathbb{H}_{X}$ as $\mathfrak{f}^{\perp}+t_{X}(\theta)$ with $\mathfrak{f}^{\perp} \in \mathbb{H}_{X}^{\perp}$. Then write the respective $\mathbb{L}_{X}^{\perp}$ and $\mathcal{L}^{2}$ components of $\mathfrak{D}_{E} \mathfrak{f}$ as

$$
\text { - }\left(1-\Pi_{X}\right) \mathfrak{D}_{E} \mathfrak{f}=\mathfrak{D}_{E}^{\perp} \mathfrak{f}^{\perp}+p_{X}(\theta) \text {. }
$$

$$
\text { - } t_{X}^{\dagger}\left(\mathfrak{D}_{E} \mathfrak{f}\right)=p_{X}{ }^{\prime}\left(\mathfrak{f}^{\perp}\right)+\Delta \theta \text {. }
$$

The lemma below says what is needed about $\mathfrak{D}_{E}^{\perp}$.

Lemma 2.6 There exists a constant $\kappa>1$ with the following significance: Fix $r \geq \kappa$ and $R \geq \kappa$ so as to define $\mathfrak{D}_{E}, \mathbb{H}_{X}^{\perp}$ and $\mathbb{L}_{X}^{\perp}$. Let $\mathfrak{D}_{E}^{\perp}$ denote the restriction of $\left(1-\Pi_{X}\right) \mathfrak{D}_{E}$ to $\mathbb{H}_{X}^{\perp}$. Then $\mathfrak{D}_{E}^{\perp}$ is a bounded, invertible map from $\mathbb{H}_{X}^{\perp}$ to $\mathbb{L}_{X}^{\perp}$ that 
obeys

$$
\kappa^{-1}\left\|\mathfrak{f}^{\perp}\right\|_{\mathbb{H}, X} \leq\left\|\mathfrak{D} \frac{\perp}{E} \mathfrak{f}^{\perp}\right\|_{2} \leq \kappa\left\|\mathfrak{f}^{\perp}\right\|_{\mathbb{H}, X}
$$

for all $\mathfrak{f}^{\perp} \in \mathbb{H}_{X}^{\perp}$.

Proof of Lemma 2.6 It follows by copying what is said in Parts 3 and 5 of the proof of Lemma 6.3 in [14] that

$$
|\mathfrak{h}| \leq c_{0} \rho_{*} e^{-\sqrt{r} \operatorname{dist}(\cdot, \Sigma) / 100} .
$$

As a consequence, the $L^{2}$ norm of the contribution to $\mathfrak{D}_{E}^{\perp} \mathfrak{f}$ from $\mathfrak{h}$ is bounded by

$$
c_{0} r^{1 / 2} \rho_{*}\left\|\mathfrak{f}^{\perp}\right\|_{2} \leq c_{0} \rho_{*}\left\|\mathfrak{f}^{\perp}\right\|_{\mathbb{H}, X} .
$$

Let $\mathfrak{D}_{E^{*}}$ denote the version of $\mathfrak{D}_{E}$ that is defined by using $\left(A_{X}^{*}, \psi_{X}^{*}\right)$ in (2-61). The latter is what is left of $\mathfrak{D}_{E}$ with the contribution from $\mathfrak{h}$ absent. A repeat of the arguments for Lemma 6.1 in [14] prove the assertion of Lemma 2.6 for $\mathfrak{D}_{E^{*}}^{\perp}$. Granted (2-65), the assertions hold for $\mathfrak{D}_{E}^{\perp}$ when $r \geq c_{0}$.

Consider next the size of $p_{X}(\theta)$ and $p_{X}\left(\mathfrak{f}^{\perp}\right)$. To bound the latter, remark first that $\mathfrak{D}_{E}$ near $\Sigma$ has the schematic form given in (6-6) and (6-7) of [14]. What with (4-7)-(4-9) in [14], these equations imply that

$$
\left\|p_{X}(\theta)\right\|_{2} \leq c_{0} r^{3 \sigma}\|\theta\|_{\mathcal{K}_{1}^{2}}
$$

Here, $\|\cdot\|_{\mathcal{K}_{1}^{2}}$ denotes the norm on $\mathcal{K}_{1}^{2}$. They also imply that

$$
\left\|p_{X}\left(\mathfrak{f}^{\perp}\right)\right\|_{\mathcal{L}^{2}} \leq c_{0} \rho_{*}\left\|\mathfrak{f}^{\perp}\right\|_{\mathbb{H}, X} \leq c_{0} r^{-1 / 2+3 \sigma}\left\|\mathfrak{f}^{\perp}\right\|_{\mathbb{H}, X}
$$

The next lemma describes $\Delta$. This lemma uses the following notation: Given finite dimensional vector spaces $V_{0}$ and $V_{1}$ with injective, linear maps $\phi_{0}: V_{0} \rightarrow \mathcal{K}_{1}^{2}$ and $\phi_{1}: V_{1} \rightarrow \mathcal{L}^{2}$, the map $\Delta$ defines a linear operator from the $\mathcal{K}_{1}^{2}$-orthogonal complement of $\phi_{0}\left(V_{0}\right)$ to the $\mathcal{L}^{2}$-orthogonal complement of $\phi_{1}\left(V_{1}\right)$ that is obtained by composing $\Delta$ with the $\mathcal{L}^{2}$-orthogonal projection. This map is denoted by $\Delta^{\perp}$.

Lemma 2.7 There exists $\kappa>1$ and finite dimensional vector spaces $V_{0}$ and $V_{1}$ with the following significance: Suppose that $r \geq \kappa$ and $R \geq \kappa$. Then the operator $\Delta: \mathcal{K}_{1}^{2} \rightarrow \mathcal{L}^{2}$ is Fredholm and its index is equal to $\operatorname{dim}\left(V_{0}\right)-\operatorname{dim}\left(V_{1}\right)$. Moreover, there exist injective linear maps $\phi_{0}: V_{0} \rightarrow \mathcal{K}_{1}^{2}$ and $\phi_{1}: V_{1} \rightarrow \mathcal{L}^{2}$ such that the associated map $\Delta^{\perp}$ is an isomorphism of vector spaces that obeys $\left\|\Delta^{\perp} \theta\right\|_{\mathcal{L}^{2}} \geq \kappa^{-1}\|\theta\|_{\mathcal{K}_{1}^{2}}$.

The proof of this lemma is given momentarily so assume it is true for now. 
What follows directly are some comments with regard to the substance of this lemma. To start, note that Lemma 2.7 has two assertions. The first is that $\Delta$ is Fredholm when $r$ is large. This assertion needs no reference to the vector spaces $V_{0}$ and $V_{1}$. These two vector spaces involve the second assertion, which is the lower bound for the norm of $\Delta^{\perp}$. There are three key points to observe with regard to this lower bound: First, the lower bound is $r$-independent. Second, the domain and range of $\Delta^{\perp}$ are subspaces with finite and $r$-independent codimension. The third point, though not stated by the lemma, is that $V_{0}, V_{1}$ can be described explicitly. This is done in the Part 3 to come. These descriptions are used to compute the index of $\Delta$. Meanwhile, Lemma 2.8 in Part 2 below asserts that the index of $\Delta$ is equal to the index of $\mathfrak{D}_{E}$.

Part 2 This part uses some straightforward linear algebra in conjunction with Lemma 2.6 to translate statements about the kernel and cokernel of $\mathfrak{D}_{E}$ into statements about the operator $\Delta$. To start this task, suppose that $\mathfrak{f} \in \mathbb{H}_{X}$ and suppose that $\mathfrak{D}_{E} \mathfrak{f}=0$. Write $\mathfrak{f}=\mathfrak{f}^{\perp}+t_{X}(\theta)$. Given Lemma 2.6, it follows from (2-64) that $\mathfrak{f}^{\perp}$ is determined by $\theta$ and can be written as $\mathfrak{f}^{\perp}=-\left(\mathfrak{D}_{E}^{\perp}\right)^{-1} p_{X}(\theta)$. This understood, then $\theta$ obeys

$$
\Delta_{E} \theta=\Delta \theta-p_{X}^{\prime}\left(\left(\mathfrak{D}_{E}^{\perp}\right)^{-1} p_{X}(\theta)\right)=0 .
$$

Equation (2-69) identifies the kernel of $\mathfrak{D}_{E}$ with the kernel of $\Delta_{E}$. With regard to $\Delta_{E}$, note that (2-67) and (2-68) find that

$$
\left\|p_{X}^{\prime}\left(\left(\mathfrak{D}_{E}^{\perp}\right)^{-1} p_{X}(\theta)\right)\right\|_{2} \leq c_{0} r^{-1 / 2+6 \sigma}\|\theta\|_{\mathcal{K}_{1}^{2}} .
$$

Now consider the cokernel of $\mathfrak{D}_{E}$. To this end, fix $\mathfrak{y} \in \mathbb{L}_{X}$. Given $\theta \in \mathcal{L}_{1}^{2}$, the equation $\mathfrak{D}_{E}^{\perp}\left(\mathfrak{f}^{\perp}+p_{X}(\theta)\right)=\mathfrak{y}^{\perp}$ is solved by

$$
\mathfrak{f}^{\perp}=-\left(\mathfrak{D} \frac{\perp}{E}\right)^{-1} p_{X}(\theta)+\left(\mathfrak{D}_{E}^{\perp}\right)^{-1} \mathfrak{y}^{\perp} .
$$

It follows that $\mathfrak{y}$ has nonzero projection to the cokernel of $\mathfrak{D}_{E}$ if and only if there is no $\theta \in \mathcal{L}_{1}^{2}$ solving

$$
\Delta_{E} \theta=p_{X}^{\prime}\left(\left(\mathfrak{D} \frac{\perp}{E}\right)^{-1} \mathfrak{y}^{\perp}\right)-t_{X}^{\dagger}(\mathfrak{y}) .
$$

Thus, the map that sends $\mathfrak{y} \in \mathbb{L}$ to the projection of $\left.p_{X}^{\prime}\left(\mathfrak{D} \frac{\perp}{E}\right)^{-1} \mathfrak{y}^{\perp}\right)-t_{X}^{\dagger}(\mathfrak{y})$ into the cokernel of $\Delta_{E}$ defines an injection from $\operatorname{cokernel}\left(\mathfrak{D}_{E}\right)$ to cokernel $\left(\Delta_{E}\right)$. Moreover, given (2-63), this injection is an isomorphism.

The following lemma summarizes:

Lemma 2.8 There exists $\kappa \geq 1$ with the following significance: Suppose that $r \geq \kappa$ and $R \geq \kappa$. Then the map $\theta \rightarrow-\left(\mathfrak{D}_{E}^{\perp}\right)^{-1} p_{X}(\theta)+t_{X}(\theta)$ and $\left.\mathfrak{y} \rightarrow p_{X}^{\prime}\left(\mathfrak{D}_{E}^{\perp}\right)^{-1} \mathfrak{y}\right)-t_{X}^{\dagger}(\mathfrak{y})$ define respective isomorphism from the kernel of $\Delta_{E}$ to the kernel of $\mathfrak{D}_{E}$ and from the cokernel of $\mathfrak{D}_{E}$ to the cokernel of $\Delta_{E}$. Thus, the operators $\mathfrak{D}_{E}$ and $\Delta_{E}$ have the same Fredholm index. Moreover, the index of the latter is the same as that $\Delta$. 
Proof of Lemma 2.8 It is necessary only to comment on the last assertion. This follows from (2-70) when $r$ is large given the assertion in Lemma 2.7 that $\left\|\Delta^{\perp} \theta\right\|_{\mathcal{L}^{2}} \geq$ $c_{0}{ }^{-1}\|\theta\|_{\mathcal{K}_{1}^{2}}$ when $\theta$ is orthogonal to $\phi_{0}\left(V_{0}\right)$.

Part 3 This part describes the vector spaces $V_{0}$ and $V_{1}$ in preparation for the proof of Lemma 2.7. There is a version for $C$ of the operator $\mathcal{D}_{C}$ that is depicted in (1-10). This operator is Fredholm as a map from $L_{1}^{2}(C ; N)$ to $L_{1}^{2}\left(C ; N \otimes T^{0,1} C\right)$. Let $V_{0 C}$ denote the kernel of $C$ 's version of this operator, and let $V_{1 C}$ denote kernel of the formal $L^{2}$ adjoint of this operator.

Now fix $(\gamma, m) \in \Theta_{-}$. Define a map $\mathfrak{c}_{\gamma-}: \mathbb{R} \times S^{1} \rightarrow \mathfrak{C}_{m}$ as follows: Let $\left\{\mathcal{E}_{1}, \ldots, \mathcal{E}_{m}\right)$ denote the set of ends of $\Sigma$ that intersect $U_{\gamma_{-}}$. Recall that each such end is a graph of a function $(w, t) \rightarrow z_{\mathcal{E}} \varsigma(t) e^{-2 \lambda w}$ where $\lambda$ is the largest negative eigenvalue of $\gamma$ 's version of (1-2) and $\varsigma(t)$ is a corresponding eigenfunction. The set $\left\{z_{\mathcal{E}_{1}}, \ldots, z_{\mathcal{E}_{m}}\right\}$ are the $m$-th roots of unity. The map $\mathfrak{c}_{\gamma-}$ is defined by specifying the $\mathbb{R} \times S^{1}$ dependence of the coordinates $\left\{\sigma_{q}\right\}_{1 \leq q \leq m}$ for $\mathfrak{C}_{m}$ that are given in (1-5) of [14]. This understood, the coordinates $\sigma_{q}$ for $q<m$ are zero and $\sigma_{m}=\left(r_{\gamma}^{1 / 2} \varsigma(t) e^{-2 \lambda w}\right)^{m}$ where $r_{\gamma}=\left(\ell_{\gamma} /(2 \pi)\right) r$ with $\ell_{\gamma}=\int_{\gamma} a$. This is to say that if $\mathfrak{c}_{\gamma}$ is written as the pair $(A, \alpha)$, then the zero locus of $\alpha$ at any given $(w, t)$ is the point in $\operatorname{Sym}^{m}(\mathbb{C})$ whose coordinates are given by $r_{\gamma}^{1 / 2} \varsigma(t) e^{-2 \lambda w}\left(z_{\mathcal{E}_{1}}, \ldots, z_{\mathcal{E}_{m}}\right)$.

Let $h: \mathfrak{C}_{m} \rightarrow \mathbb{R}$ denote $\gamma$ 's version of (1-6) and introduce the operator

$$
\zeta \rightarrow \bar{\partial} \zeta+\left.\left(\nabla_{\zeta_{\mathbb{R}}} \nabla^{1,0} h\right)\right|_{\mathfrak{c}_{\mathcal{\gamma}}},
$$

a linear map from $L_{1}^{2}\left(\mathbb{R} \times S^{1} ; \mathfrak{c}_{\gamma-}{ }^{*} T_{1,0} \mathfrak{C}_{m}\right)$ to $L^{2}\left(\mathbb{R} \times S^{1} ; \mathfrak{c}_{\gamma-}{ }^{*} T_{1,0} \mathfrak{C}_{m}\right)$. As explained next, the assumptions in (2-55) imply that this operator is Fredholm. To see this, suppose first that $m=1$. Use the function $\sigma_{1}$ in (1-5) of [14] to identify $\mathfrak{C}_{1}$ with $\mathbb{C}$. Under this identification, the operator in (2-73) acts on the space of $\mathbb{C}$-valued functions on $\mathbb{R} \times S^{1}$ as the operator $\bar{\partial}+v+\mu \overline{(\cdot)}$. The assumptions in (2-55) imply that this is Fredholm as a map from $L_{1}^{2}\left(\mathbb{R} \times S^{1} ; \mathfrak{c}_{\gamma-}{ }^{*} T_{1,0} \mathfrak{C}_{m}\right)$ to $L^{2}\left(\mathbb{R} \times S^{1} ; \mathfrak{c}_{\gamma-}{ }^{*} T_{1,0} \mathfrak{C}_{m}\right)$.

Consider next the case $m \geq 1$. To start, introduce the basis vectors $\left\{\partial / \partial \sigma_{q}\right\}_{1 \leq q \leq m}$ for $T_{1,0} \mathfrak{C}_{m}$ that are dual to the coordinates $\left\{\sigma_{q}\right\}_{1 \leq q \leq m}$ in (1-5) of [14]. With $\zeta$ written as $\sum_{1 \leq q \leq m} \zeta_{q}\left(\partial / \partial \sigma_{q}\right)$, the operator in (2-73) acts diagonally to send $\zeta_{q}$ to $\bar{\partial} \zeta_{q}+(q / 2) \mathrm{R} \zeta_{q}$, where $\mathrm{R}$ is the rotation number that appears in (2-55). This is because the relevant version of $\xi$ lacks the term with $\mu$ and so the corresponding Hamiltonian vector field in question is $\frac{1}{2} R$ times the generator of the $S^{1}$ action on $\mathfrak{C}_{m}$.

Introduce next the function $w \rightarrow \rho(w)=r_{\gamma}^{1 / 2} e^{-2 \lambda w}$ Note that in this $m>1$ case, $\lambda$ is equal to $\frac{1}{2}\left(\mathrm{R}-k_{\mathrm{R}}^{>}\right)$where $k_{\mathrm{R}}^{>}$is the least integer greater than $\mathrm{R}$. The inner product 
between the $\mathfrak{c}_{\gamma-}$-pullbacks of the dual basis $\left\{d \sigma_{q}\right\}_{1 \leq q \leq m}$ is diagonal, and such that

$$
\left\langle\mathfrak{c}_{\gamma-}{ }^{*} d \sigma_{q}, \mathfrak{c}_{\gamma-}{ }^{*} d \sigma_{q}\right\rangle=c_{*} \rho^{2 q-2} q^{2}+\mathfrak{m}_{q},
$$

where $c_{*}>0$ and where $\left|\mathfrak{m}_{q}\right| \leq c_{0}$ is asymptotic as $w \rightarrow-\infty$ to a positive constant. The diagonal form for the metric follows because the set $\left\{z_{\mathcal{E}_{1}}, \ldots, z_{\mathcal{E}_{m}}\right\}$ are the $m-$ th roots of unity. With $\mathfrak{c}_{\gamma-}{ }^{*} T_{1,0} \mathfrak{C}_{m}$ identified with $\mathbb{R} \times S^{1} \times \mathbb{C}^{m}$ using the basis $\left\{\partial / \partial \sigma_{q}\right\}_{1 \leq q \leq m}$, write an element in the $q$-th summand as $\eta(1+\rho)^{q-1}\left(\partial / \partial \sigma_{q}\right)$. By (2-74), this element has norm $\left|\mathfrak{n}_{q}\right||\eta|$ with $c_{0}{ }^{-1} \leq\left|\mathfrak{n}_{q}\right| \leq c_{0}$ where $\mathfrak{n}_{q}$ has constant limits as $s \rightarrow \pm \infty$. The operator $\bar{\partial}+(q / 2) \mathrm{R}$ acts on such an element so as to send $\eta$ to

$$
\bar{\partial} \eta+\frac{1}{2}\left(q \mathrm{R}+(q-1) \rho /(1+\rho)\left(k_{\mathrm{R}}^{>}-\mathrm{R}\right)\right) \eta .
$$

This operator looks like

$$
\text { - } \bar{\partial}+\frac{q}{2} \mathrm{R} \text { for } w \ll-1 \text {. }
$$

$$
\text { - } \bar{\partial}+\frac{1}{2}\left(\mathrm{R}+(q-1) k_{\mathrm{R}}^{>}\right) \text {for } w \gg 1 \text {. }
$$

Given what is assumed in (2-55), it follows from (2-76) that each $q \in\{1, \ldots, m\}$ version of (2-75) is Fredholm. As a consequence, the operator (2-73) is Fredholm as a map from $L_{1}^{2}\left(\mathbb{R} \times S^{1} ; \mathfrak{c}_{\gamma-}{ }^{*} T_{1,0} \mathfrak{C}_{m}\right)$ to $L^{2}\left(\mathbb{R} \times S^{1} ; \mathfrak{c}_{\gamma-}{ }^{*} T_{1,0} \mathfrak{C}_{m}\right)$. Moreover, its index is nonnegative and its cokernel is trivial. Indeed, the arguments for this are, but for notation, identical to those used in [14] for Step 2 of the proof of Lemma 7.5 subsequent to (7-61) in [14]. Let $V_{0 \gamma-}$ to denote the kernel of this same version of (2-73).

In the case when $(\gamma, m) \in \Theta_{+}$, there is an analog of (2-75) that is obtained by replacing $k_{\mathrm{R}}^{>}$where it appears explicitly and in the definition of $\rho$ with $k_{\mathrm{R}}^{<}$, this the greatest integer that is less than $\mathrm{R}$. The resulting analog of (2-76) is such that the top line holds where $w \gg 1$ and the lower line with $k_{\mathrm{R}}^{<}$replacing $k_{\mathrm{R}}^{>}$holds where $w \ll-1$. It then follows from (2-55) that the $\mathfrak{c}_{\gamma}+$ version of (2-73) is Fredholm with trivial cokernel.

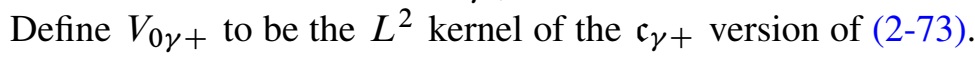

Granted these definitions, set

$$
\begin{aligned}
& V_{0}=\left(\bigoplus_{C \in \mathcal{C}} V_{0 C}\right) \oplus\left(\bigoplus_{(\gamma, m) \in \Theta} V_{0 \gamma-}\right) \oplus\left(\bigoplus_{(\gamma, m) \in \Theta^{\prime}} V_{0 \gamma-}\right) . \\
& V_{1}=\bigoplus_{C \in \mathcal{C}} V_{1 C} .
\end{aligned}
$$

These are the spaces $V_{0}$ and $V_{1}$ in Lemma 2.7 .

Part 4 This part constitutes a digression to prepare for the proof of Lemma 2.7. To start, let $\mathfrak{D}_{E^{*}}$ again denote the version of $\mathfrak{D}_{E}$ that is defined using the pair $\left(A_{X}^{*}, \psi_{X}^{*}\right)$ in (2-61). The latter is what is left of $\mathfrak{D}_{E}$ with the contribution from $\mathfrak{h}$ absent. Recall that $\mathfrak{h}$ is defined in (2-60) and that $\left(A_{X}, \psi_{X}\right)$ is obtained from $\left(A_{X}^{*}, \psi_{X}^{*}\right)$ by adding 
terms from $\mathfrak{h}$. What follows describes certain aspects of $\mathfrak{D}_{E^{*}}$ on each $U_{C}$ and on each $U_{\gamma \pm}$.

Suppose that $C \subset \mathcal{C}$ denotes a given component. A somewhat more detailed analysis of $\mathfrak{D}_{E^{*}}$ on $U_{C}$ writes the contribution to the $\mathfrak{D}_{E^{*}}$ version of (6-6) in [14] from a section $\mathfrak{f}_{0}=\left(q, \eta_{0}\right)$ of $\mathbb{V}_{C 0}$ as

$$
\begin{array}{ll}
- & 2\left(\bar{\partial}_{\theta}^{H} q-\left(v_{*} \mathfrak{s}+\mu_{*} \overline{\mathfrak{s}}\right) \partial^{V} q+\left(v_{*} \overline{\mathfrak{s}}+\bar{\mu}_{*} \mathfrak{s}\right) \bar{\partial}^{v} q+v_{*} q+\mu_{*} \bar{q}\right)+\mathfrak{r}_{* 0} . \\
\text { - } & 2\left(\bar{\partial}_{A_{X}^{*}}^{H} \eta_{0}-\left(v_{*} \mathfrak{s}+\mu_{*} \overline{\mathfrak{s}}\right) \partial_{A_{X}^{*}}^{V} \eta_{0}+\left(v_{*} \overline{\mathfrak{s}}+\bar{\mu}_{*} \mathfrak{s}\right) \bar{\partial}_{A_{X}^{*}}^{V} \eta_{0}\right)_{0}+\mathfrak{r}_{* 1} .
\end{array}
$$

Here, $\left|\mathfrak{r}_{* 0}\left(\mathfrak{f}_{0}\right)\right|+\left|\mathfrak{r}_{* 1}\left(\mathfrak{f}_{0}\right)\right| \leq c_{0}\left(|\mathfrak{s}|\left(\left|\mathfrak{f}_{0}\right|+\left|\nabla^{H} \mathfrak{f}_{0}\right|\right)+|\mathfrak{s}|^{2}\left|\nabla \mathfrak{f}_{0}\right|\right)$ with $\nabla$ the derivative that is defined by $A_{X}^{*}$ and with $\nabla^{H}$ denoting the derivative along horizontal tangent vectors in the normal bundle to $C$. In this version of (2-78), the pair $\left(v_{*}, \mu_{*}\right)=$ $\left(v_{C}, \mu_{C}\right)$.

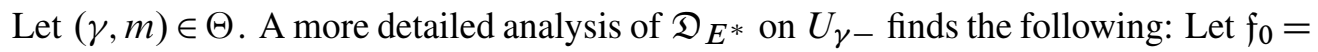
$\left(q, \eta_{0}\right)$ denote a map to $\mathbb{V}_{0}$ over the part of $(-\infty,-R) \times S^{1} \times \mathbb{C}$ where $|Z| \leq \rho_{*}$. Then the $U_{\gamma-}$ analog of (6-6) in [14] for $\mathfrak{D}_{E^{*}}$ is given up to inconsequential factors of $2 \pi / \ell_{\gamma}$ by (2-78) with the identifications $\mathfrak{s}=z, \partial^{V}=\partial / \partial z$, and $\partial_{\theta}^{H}=\frac{1}{2}(\partial / \partial w-i(\partial / \partial t))$. Meanwhile what is written as $\mathfrak{r}_{* 0,1}$ obey $\left|\mathfrak{r}_{* 1}\left(\mathfrak{f}_{0}\right)\right|+\left|\mathfrak{r}_{* 0}\left(\mathfrak{f}_{0}\right)\right| \leq c_{0}\left(|z|\left(\left|\mathfrak{f}_{0}\right|+\left|\nabla^{H} \mathfrak{f}_{0}\right|\right)+\right.$ $\left.|z|^{2}\left|\nabla \mathfrak{f}_{0}\right|\right)$. In this version, $\left(\nu_{*}, \mu_{*}\right)$ are the pair $(\nu, \mu)$ associated to $\gamma$. There is an analogous picture of $\mathfrak{D}_{E^{*}}$ on $U_{\gamma+}$ for $(\gamma, m) \in \Theta^{\prime}$.

Part 5 This part describes the operator $\Delta$ in preparation for the proof of Lemma 2.7. To start, fix $\theta=\left(\left(\theta_{c}\right)_{C \in \mathcal{C}},\left(\theta_{\gamma-}\right)_{(\gamma, m) \in \Theta},\left(\theta_{\gamma+}\right)_{(\gamma, m) \in \Theta^{\prime}}\right) \in \mathcal{K}_{1}^{2}$ and write the components of $\Delta \theta$ as $\left(\left(\Delta_{C}\right)_{C \in \mathcal{C}},\left(\Delta_{\gamma-}\right)_{(\gamma, m) \in \Theta},\left(\Delta_{\gamma+}\right)_{(\gamma, m) \in \Theta^{\prime}}\right)$. Consider $\Delta_{C}$ for $C \in \mathcal{C}$ first. View $\theta_{C}$ as a section of $C$ 's normal bundle, $N$, and view $\Delta_{C}$ as a section of $N \otimes T^{0,1} C$. Use $t_{X}(\theta)$ to define $\mathfrak{f}_{0}$ in $C$ 's version of $(2-78)$, in all $(\gamma, m) \in \Theta_{-}$ versions for the cases where $C \cap U_{\gamma-} \neq \varnothing$, and in all $(\gamma, m) \in \Theta_{+}$versions for the cases where $C \cap U_{\gamma+} \neq \varnothing$. Doing the relevant integrals to compute $t_{X}^{\dagger}\left(\mathfrak{D}_{E^{*} t_{X}}(\theta)\right)$ using (6-6)-(6-7) in [14] finds that the $\mathfrak{D}_{E^{*}}$ contribution to $\Delta_{C}$ has the form

$$
\bar{\partial} \theta_{C}+v_{C} \theta_{C} \frac{1}{\pi} \int_{\mathbb{C}}\left|\iota_{1}\right|^{2}+\mu_{C} \bar{\theta}_{C} \frac{1}{\pi} \int_{\mathbb{C}}\left|x_{1}\right|^{2}+\mathfrak{e},
$$

where $\left(x_{1}, \iota_{1}\right)=\left(2^{-1 / 2}\left(1-|\alpha|^{2}\right), \partial_{A} \alpha\right)$ with $(A, \alpha)$ here denoting the symmetric vortex in $\mathfrak{C}_{1}$. Meanwhile, the $L^{2}$ norm of $\mathfrak{e}$ obeys $\|\mathfrak{e}\|_{\mathcal{L}^{2}} \leq c_{0} r^{-\sigma / 4}\|\theta\|_{\mathcal{K}_{1}^{2}}$ Finally, $\nu_{C}$ and $\mu_{C}$ are the respective sections of $T^{0,1} C$ and $N^{\oplus 2} \otimes T^{0,1} C$ that appear in (1-10). Granted (2-79) and the definition of $\mathfrak{h}$, the bounds from Parts 3 and 5 of the proof of Lemma 6.3 in [14] on the various terms in (2-60) imply that

$$
\Delta_{C}(\theta)=\bar{\partial} \theta_{C}+v_{C} \theta_{C}+\mu_{C} \bar{\theta}_{C}+\mathfrak{e},
$$

where the $L^{2}$ norm of $\mathfrak{e}$ on the $|s| \leq 2 R$ part of $C$ is bounded by $c_{0} r^{-\sigma / 4}\|\theta\|_{\mathcal{K}_{1}^{2}}$. 
Now fix $(\gamma, m) \in \Theta$ so as to consider $\Delta_{\gamma-}$. The case considered first is that where $m=1$. Thus, there is one end of $\Sigma$ in $U_{\gamma}$. This end is denoted by $\mathcal{E}$. To describe $\Delta_{\gamma-}$, it proves convenient to view both $\Delta_{\gamma-}(\theta)$ and $\theta_{\gamma-}$ as maps from $(-\infty,-R) \times S^{1}$ to $\mathbb{C}$. This is done using the coordinate $\sigma_{1}$ from (1-5) of [14]. Use $t_{X}(\theta)$ to define the element $\mathfrak{f}_{0}=\left(q, \eta_{0}\right)$ in the $(\gamma, m)$ version of (2-78) and in the versions that are defined by the element $C \in \mathcal{C}$ that contains $\mathcal{E}$. Doing the relevant integrals to compute $t_{X}^{\dagger}$ finds that $\Delta_{\gamma-}(\theta)$ is given by

$$
\Delta_{\gamma-}(\theta)=\bar{\partial} \theta_{\gamma-}+\nu \theta_{\gamma-}+\mu \bar{\theta}_{\gamma-}+\mathfrak{e},
$$

where the $L^{2}$ norm of $\mathfrak{e}$ on the $s \leq-R$ part of $U_{\gamma}$ is bounded by $c_{0} r^{-\sigma / 4}\|\theta\|_{\mathcal{K}_{1}^{2}}$ Indeed, (2-81) follows using Lemma 2.2 and the arguments that lead (2-35). The following are the only substantive changes: First, $\mathfrak{u}$ in (2-32) now depends on the variables $w$ and $t$ for $(-\infty,-R) \times S^{1}$. To describe $\mathfrak{u}$, recall that $\mathcal{E}$ appears as the graph in $(-\infty,-R) \times S^{1} \times \mathbb{C}$ of the function $(w, t) \rightarrow \varsigma(t) e^{-2 \lambda w}$ where $\lambda$ and $\varsigma(t)$ are as described in Part 1 of Section 2.b.

Granted the preceding, $\mathfrak{u}=\vartheta_{0}^{\dagger}(y, 0)$ with $y=2^{1 / 2}\left(z-z_{\gamma-}\right) \alpha^{-1} \partial_{A} \alpha$ and $\left.z_{\gamma-}\right|_{(w, t)}=$ $r_{\gamma}^{1 / 2} z_{\mathcal{E}} e^{-2 \lambda w}$; and with $\left.(A, \alpha)\right|_{(w, t)}$ denoting the vortex in $\mathfrak{C}_{1}$ with $\alpha^{-1}(0)=z_{\gamma}$. Meanwhile, what is called $\mathfrak{w}$ in (2-32) is replaced with $\left(0,\left(\bar{z}_{\gamma_{-}}\right) \bar{\alpha}^{-1}\left(1-|\alpha|^{2}\right)\right)$ with $z_{\gamma-}$ and $\alpha$ just described.

Turn now to the case where $m>1$. In this case,

$$
\Delta_{\gamma-}(\theta)=\bar{\partial} \theta_{\gamma-}+\left.\left(\nabla_{\left(\theta_{\gamma-}\right)_{\mathbb{R}}} \nabla^{1,0} \hbar\right)\right|_{c_{\gamma-}}+\mathfrak{e},
$$

where $\mathfrak{e}$ has $L^{2}$ norm bounded by $c_{0} r^{-\sigma / 4}\|\theta\|_{\mathcal{K}_{1}^{2}}$. The proof of (2-82) is identical, but for notation, to the proof of the formula for $\mathrm{D} \theta$ that is given in Lemma 2.1.

There is, of course, an analogous description of $\Delta_{\gamma+}$ when $(\gamma, m) \in \Theta^{\prime}$.

Part 6 This part contains the promised.

Proof of Lemma 2.7 The fact that $\Delta$ is Fredholm with the asserted index follows from the assertions made about $\Delta^{\perp}$. The proof of these assertions borrows much from Section 7.f in [14]. There are five steps.

Step 1 This step defines the map $\phi_{0}: V_{0} \rightarrow \mathcal{K}_{1}^{2}$. To start, fix $C \in \mathcal{C}$ and $\eta \in V_{0 C}$. Write the components of $\phi_{0}(\eta)$ as $\left(\left(\phi_{0 C^{\prime}}\right)_{C^{\prime} \in \mathcal{C}},\left(\phi_{0 \gamma-}\right)_{(\gamma, m) \in \Theta},\left(\phi_{0 \gamma+}\right)_{(\gamma, m) \in \Theta^{\prime}}\right)$ with the $\eta$-dependence of these components implicit in what follows. These components are defined as follows: First, $\phi_{0 C^{\prime}}=0$ unless $C^{\prime}=C$. Meanwhile, $\phi_{0 C}=\left(1-\Sigma_{\mathcal{E} \in \mathcal{E}_{C}} \chi_{\mathcal{E}}^{\prime}\right) \eta$ where $\mathcal{E}_{C}$ and $\chi_{\mathcal{E}}^{\prime}$ are defined in the following manner: First, $\mathcal{E}_{C}$ denotes the set of ends of $C$. Second, the cut-off function $\chi_{\mathcal{E}}^{\prime}$ is equal zero on the complement of the 
end $\mathcal{E}$; and it is equal to $\chi\left(\left(R_{\mathcal{E}}+3.5 R-|w|\right) / R\right)$ on $\mathcal{E}$. Each $(\gamma, m) \in \Theta_{+}$version of $\phi_{0 \gamma-}$ and each $(\gamma, m) \in \Theta_{+}$version of $\phi_{0 \gamma+}$ is determined from $\phi_{0 C}$ via the homomorphisms in (5-12) in [14].

To continue, fix $(\gamma, m) \in \Theta_{-}$and suppose that $\eta \in V_{0 \gamma}$. The components of $\phi_{0}(\eta)$ are defined in this case as follows: First, $\phi_{0 \gamma^{\prime}-}=0$ unless $\gamma=\gamma^{\prime}$ in which case $\phi_{0 \gamma-}=\chi_{R-}^{\prime} \eta$. Here, $w \rightarrow \chi_{R-}^{\prime}(w)=\chi((2.5 R+w) / R)$. Meanwhile, each $C \in \mathcal{C}$ version of $\phi_{0 C}$ is zero unless $C$ intersects $U_{\gamma-}$ in which case $\phi_{0 C}$ is determined from $\phi_{0 \gamma-}$ via the homomorphisms in (5-12) of [14]. Finally, each $(\gamma, m) \in \Theta^{\prime}$ version of $\phi_{0 \gamma+}$ is zero. There is an analogous definition of $\phi_{0}(\eta)$ in the case when $\eta$ is in some $(\gamma, m) \in \Theta^{\prime}$ version of $V_{0 \gamma+}$.

It is an exercise to verify that

$$
\left(1-c_{0} \delta\right)\|\eta\|_{L_{1}^{2}} \leq\left\|\phi_{0}(\eta)\right\|_{\mathcal{K}_{1}^{2}} \leq\left(1+c_{0} \delta\right)\|\eta\|_{L_{1}^{2}}
$$

where $\delta$ here and in what follows is short hand for $\delta=R^{-1}+r^{-1 / c_{0}}$. Thus, $\phi_{0}$ is injective when $r \geq c_{0}$ and $R \geq c_{0}$. Note in this regard that if $C \in \mathcal{C}$ and if $\eta \in V_{0 C}$, then

$$
|\eta|+|\nabla \eta| \leq c_{0}\|\eta\|_{L^{2}} e^{-|s| / c_{0}} .
$$

By the same token, if $(\gamma, m) \in \Theta$ and $\eta \in V_{0 \gamma-}$, then

$$
|\eta|+|\nabla \eta| \leq c_{0}\|\eta\|_{L^{2}} e^{-|w+(\ln r) / 4 \lambda| c_{0}} .
$$

In the case $(\gamma, m) \in \Theta_{+}$and $\eta \in V_{\gamma+}$, there is an analogous inequality, this obtained from (2-85) by changing $w$ to $-w$. Meanwhile, it follows from the description of $\Delta$ in Part 5 that

$$
\left\|\Delta \phi_{0}(\eta)\right\|_{\mathcal{L}^{2}} \leq c_{0} \delta\|\eta\|_{L_{1}^{2}}
$$

when $\eta \in V_{0}$ and $r \geq c_{0}$ and $R \geq c_{0}$.

Step 2 This step proves that $\|\Delta \theta\|_{\mathcal{L}^{2} \geq c_{0}}{ }^{-1}\|\theta\|_{\mathcal{K}_{1}^{2}}$ when $\theta$ is $\mathcal{K}_{1}^{2}$ orthogonal to the image of $\phi_{0}$ provided that $r \geq c_{0}$ and $R \geq c_{0}$. To this end, fix $\theta \in \mathcal{K}_{1}^{2}$ and decompose it as

$$
\theta=\sum_{C \in \mathcal{C}} \theta^{c}+\sum_{(\gamma, m) \in \theta} \theta^{\gamma-}+\sum_{(\gamma, m) \in \theta^{\prime}} \theta^{\gamma+}
$$

by copying what is done in (7-44) of [14]. The terms here are determined by their namesake components of $\theta=\left(\left(\theta_{C}\right)_{C \in \mathcal{C}},\left(\theta_{\gamma-}\right)_{(\gamma, m) \in \theta},\left(\theta_{\gamma+}\right)_{(\gamma, m) \in \theta^{\prime}}\right)$. Consider $\theta^{C}$. Since $\theta^{C} \in \mathcal{K}_{1}^{2}$, it has components $\theta^{C}=\left(\left(\theta_{C^{\prime}}^{C}\right)_{C^{\prime} \in \mathcal{C}},\left(\theta_{\gamma-}^{C}\right)_{(\gamma, m) \in \theta},\left(\theta_{\gamma+}^{C}\right)_{(\gamma, m) \in \theta^{\prime}}\right)$. These are as follow: First, $\theta_{C^{\prime}}^{C}=0$ unless $C=C^{\prime}$. In this case, $\theta_{C}^{C}=\left(1-\Sigma_{\mathcal{E} \in \mathcal{E} \chi_{\mathcal{E}}}\right) \theta_{C}$ 
where the product is indexed by the ends in $C$. Here, $\chi_{\mathcal{E}}$ is defined so as to equal zero on the complement of $\mathcal{E}$ and to equal $\chi\left(\left(R_{\mathcal{E}}+3 R-|w|\right) / R\right)$ on $\mathcal{E}$. Meanwhile, any given $\theta_{\gamma \pm}^{C}$ is determined by $\left(\theta_{C^{\prime}}^{C}\right)_{C^{\prime} \in \mathcal{C}}$ through the homomorphism $\phi_{\gamma \pm}$ given in (5-12) in [14].

As an element of $\mathcal{K}_{1}^{2}$, the term $\theta^{\gamma-}$ from (2-83) has only nonzero components $\theta_{\gamma-}^{\gamma-}$ and $\theta_{C}^{\gamma-}$ for those $C$ with $C \cap U_{\gamma-} \neq \varnothing$. In particular, $\theta_{\gamma^{\prime}-}^{\gamma-}=0$ if $\gamma^{\prime} \neq \gamma$, and also $\theta_{\gamma^{\prime}+}^{\gamma-}=0$ for all $\gamma^{\prime} \in \Xi_{\Sigma+}$. The component $\theta_{\gamma-}^{\gamma-}$ is set equal to $\theta_{\gamma-}-\sum_{\gamma^{\prime}} C_{C} \cap U_{\gamma^{\prime}} \neq \varnothing \theta_{\gamma-}^{C}$. Note $\theta_{\gamma-}^{\gamma-}$ has support where $w<-3 R$. Meanwhile, a component $\theta_{C}^{\gamma}-$ is determined from $\theta_{\gamma-}^{\gamma-}$ by the identifications in (5-12) of [14]. Each $\theta^{\gamma+}$ for $(\gamma, m) \in \Theta_{+}$has a similar description.

Given the description of $\Delta$ in Part 5 and given the description of the decomposition in (2-87), the argument for (7-44) in [14] can be repeated to prove that

$$
\begin{aligned}
\|\Delta(\theta)\|_{\mathcal{L}^{2}} \geq c_{0}{ }^{-1}\left(\sum_{C \in \mathcal{C}}\left\|\Delta\left(\theta^{C}\right)\right\|_{\mathcal{L}^{2}}\right. & +\sum_{(\gamma, m) \in \Theta}\left\|\Delta\left(\theta^{\gamma-}\right)\right\|_{\mathcal{L}^{2}} \\
& \left.+\sum_{(\gamma, m) \in \Theta^{\prime}}\left\|\Delta\left(\theta^{\gamma+}\right)\right\|_{\mathcal{L}^{2}}\right)-c_{0} \delta\|\theta\|_{\mathcal{K}_{1}^{2}}
\end{aligned}
$$

when $r \geq c_{0}$ and $R \geq c_{0}$.

It is a consequence of (2-79) that $\left\|\Delta\left(\underline{\theta}^{C}\right)\right\|_{\mathcal{L}^{2}} \geq c_{0}{ }^{-1}\left\|\theta^{C}\right\|_{\mathcal{K}_{1}^{2}}$ if $\theta_{C}^{C}$ is $L_{1}^{2}$ orthogonal to the $L^{2}$ kernel of $\bar{\partial}+v_{C}+\mu_{C}\left(\overline{(\cdot)}\right.$ when $r \geq R^{3}$ and $R \geq c_{0}$. Meanwhile, if $(\gamma, m) \in \Theta_{-}$, then $\left\|\Delta\left(\theta^{\gamma-}\right)\right\|_{\mathcal{L}^{2}} \geq c_{0}{ }^{-1}\left\|\theta^{\gamma-}\right\|_{\mathcal{K}_{1}^{2}}$ when $\theta_{\gamma-}^{\gamma-}$ is $L_{1}^{2}$ orthogonal to the space $V_{0 \gamma-}$ when $r$ and $R$ obey similar bounds. And, if $(\gamma, m) \in \Theta_{-}$, then $\left\|\Delta\left(\theta^{\gamma+}\right)\right\|_{\mathcal{L}^{2}} \geq c_{0}{ }^{-1}\left\|\theta^{\gamma+}\right\|_{\mathcal{K}_{1}^{2}}$ if $\theta_{\gamma+}^{\gamma+}$ is $L_{1}^{2}$ orthogonal to the space $V_{0 \gamma+}$ given that $r \geq c_{0}$ and $R \geq c_{0}$.

These last assertions with (2-83)-(2-85) and (2-88) imply the desired result: If $r \geq c_{0}$ and $R \geq c_{0}$, then $\|\Delta(\theta)\|_{\mathcal{L}^{2}} \geq c_{0}{ }^{-1}\|\theta\|_{\mathcal{K}_{1}^{2}}$ when $\theta$ is $L_{1}^{2}$ orthogonal to the image of $\phi_{0}$.

Step 3 The map $\phi_{1}$ is defined by mimicking the definition of $\phi_{0}$. Given that (2-84) holds for $\eta \in V_{1 C}$ when $C \in \mathcal{C}$, it follows that

$$
\left(1-c_{0} \delta\right)\|\eta\|_{L^{2}} \leq\left\|\phi_{1}(\eta)\right\|_{\mathcal{L}^{2}} \leq\left(1+c_{0} \delta\right)\|\eta\|_{L^{2}} .
$$

Note that there is an approximate inverse to the map $\phi_{1}$. This is a map, $q_{1}: \mathcal{L}^{2} \rightarrow V_{1}$, that is defined as follows: Decompose any given $\theta \in \mathcal{L}^{2}$ as in (2-87). Suppose that $C \in \mathcal{C}$ and $\eta \in V_{1 C}$. The inner product of $\eta$ with $q_{1}(\theta)$ is by definition equal to the $L^{2}$ inner product of $\theta_{C}^{C}$ with $\eta$. The $V_{1 C}$ version of (2-84) implies that $\left\|q_{1} \phi_{1}(\eta)-\eta\right\|_{L^{2}} \leq c_{0} \delta \mid \eta \|_{L^{2}}$ when $r \geq c_{0}$ and $R \geq c_{0}$. 
Step 4 This step verifies the lemma's assertion about the norm of $\Delta^{\perp} \theta$ when $r \geq c_{0}$ and $R \geq c_{0}{ }^{-1}$. To this end, consider first the $\mathcal{L}^{2}$ orthogonal projection of $\Delta \theta$ onto $\phi_{1}\left(V_{1}\right)$. Integration by parts with what is said about $\Delta$ in Part 5 and the $V_{1}$ analogs of (2-84)-(2-86) can be used to prove that

$$
\left.\|\left(\Delta-\Delta^{\perp}\right)(\theta)\right)\left\|_{L^{2}} \leq c_{0} \delta\right\| \theta \|_{\mathcal{K}_{1}^{2}}
$$

when $r \geq c_{0}$ and $R \geq c_{0}$. What with the final conclusion of Step 2, this last bound implies that

$$
\left\|\Delta^{\perp} \theta\right\|_{\mathcal{L}^{2}} \geq c_{0}{ }^{-1}\|\theta\|_{\mathcal{K}_{1}^{2}}
$$

when $\theta$ is $\mathcal{K}_{1}^{2}$ orthogonal to $\phi_{0}\left(V_{0}\right)$. Here it is again assumed that $r \geq c_{0}$ and $R \geq c_{0}$. This last conclusion implies that $\Delta^{\perp}$ is injective.

Step 5 To see that $\Delta^{\perp}$ is surjective, note first that it follows from (2-91) that $\Delta^{\perp}$ has closed range. Thus, $\Delta^{\perp}$ is not surjective if and only if the $L^{2}$ orthogonal complement of its image has positive dimension. Let $\eta$ denote an element in this orthogonal complement. Introduce the map $q_{1}: \mathcal{L}^{2} \rightarrow V_{1}$ from Step 3 and write $\eta=\eta^{\prime}+\eta^{\prime \prime}$ where $\eta^{\prime}$ is the $L^{2}$-orthogonal projection of $\eta$ into the kernel of $q_{1}$. Note that $\eta^{\prime \prime}$ satisfies $\left\|\eta^{\prime \prime}\right\|_{\mathcal{L}^{2}} \leq c_{0} R^{-2}\|\eta\|_{\mathcal{L}^{2}}$. Indeed, this follows from what is said about $q_{1}$ in Step 4 and from the $V_{1}$ analogs of (2-83)-(2-85). Granted this, it is sufficient to prove that $\eta^{\prime}=0$ when $R$ and $r$ are large. To this end, decompose $\eta^{\prime}=\sum_{C \in \mathcal{C}} \eta^{C}+\sum_{(\gamma, m) \in \Theta} \eta^{\gamma-}+$ $\Sigma_{(\gamma, m) \in \Theta^{\prime}} \eta^{\gamma+}$ as was done with $\theta$ in (2-87).

Fix $C \in \mathcal{C}$ and consider the term $\eta^{C}=\left(\left(\eta_{C^{\prime}}^{C}\right)_{C^{\prime} \in \mathcal{C}},\left(\eta_{\gamma-}^{C}\right)_{(\gamma, m) \in \Theta},\left(\eta_{\gamma+}^{C}\right)_{(\gamma, m) \in \Theta^{\prime}}\right)$. Here are two observations that play a key role: First,

$$
\left\|q_{1}\left(\eta^{C}\right)\right\|_{L^{2}} \leq c_{0} \delta\left\|\eta^{\prime}\right\|_{\mathcal{L}^{2}}
$$

when $r \geq c_{0}$ and $R \geq c_{0}$. Indeed, this follows because $\eta_{C}^{C}$ is $L^{2}$ orthogonal to $V_{1 C}$.

Here is the second observation: Because $\eta_{C}^{C}$ is $L^{2}$ orthogonal to $V_{1 C}$, there exists an element, $\xi_{C} \in L_{1}^{2}(C ; N)$ that is mapped to $\eta_{C}^{C}$ by $\bar{\partial}+v_{C}+\mu_{C}(\bar{\cdot})$. Moreover, $\xi_{C}$ is $L^{2}$ orthogonal to $V_{1 C}$ and is such that

$$
c_{0}^{-1}\left\|\eta_{C}^{C}\right\|_{L^{2}} \leq\left\|\xi_{C}\right\|_{\mathcal{K}_{1}^{2}} \leq c_{0}\left\|\eta_{C}^{C}\right\|_{L^{2}}
$$

Define an element $\xi^{C}=\left(\left(\xi_{C^{\prime}}^{C}\right)_{C^{\prime} \in \mathcal{C}},\left(\xi_{\gamma-}^{C}\right)_{(\gamma, m) \in \Theta},\left(\xi_{\gamma+}^{C}\right)_{(\gamma, m) \in \Theta^{\prime}}\right) \in \mathcal{K}_{1}^{2}$ as follows: First, $\xi_{C^{\prime}}^{C}=0$ unless $C=C^{\prime}$ in which case

$$
\xi_{C}^{C}=\left(1-\Sigma_{\mathcal{E} \in \mathcal{E}_{C}} \chi_{\mathcal{E}}^{\prime}\right) \xi_{C}
$$


Here, as in Step 1, $\chi_{\mathcal{E}}^{\prime}$ is equal zero on the complement of the end $\mathcal{E}$; and it is equal to $\chi\left(\left(R_{\mathcal{E}}+3.5 R-|w|\right) / R\right)$ on $\mathcal{E}$. Meanwhile, each $(\gamma, m) \in \Theta$ version of $\xi_{\gamma-}^{C}$ and each $(\gamma, m) \in \Theta^{\prime}$ version of $\xi_{\gamma+}^{C}$ is determined from $\xi_{C}^{C}$ via the homomorphisms in (5-12) in [14]. It follows from (2-4)-(2-5) of [14] and from what is said in Part 5 about $\Delta$ that

$$
\left\|\Delta \xi^{C}-\eta^{C}\right\|_{\mathcal{L}^{2}} \leq c_{0} \delta\left\|\eta^{C}\right\|_{\mathcal{L}^{2}}
$$

This is a consequence of the fact that $\left(1-\chi_{\mathcal{E}}\right)=0$ where $d \chi_{\mathcal{E}}^{\prime} \neq 0$. Here, $\chi_{\mathcal{E}}$ is the cut-off function $\chi\left(\left(R_{E}+3 R-|w|\right) / R\right)$ defined just prior to (5-9) in [14].

Now let $\xi^{C \perp}$ denote the $L^{2}$ orthogonal projection of $\xi^{C}$ to the complement of the image of $\phi_{0}$. It is a consequence of (2-84), (2-85) and its $w \rightarrow-w$ analog for when $(\gamma, m) \in \Theta_{+}$that $\left\|\xi^{C \perp}-\xi^{C}\right\|_{L_{1}^{2}} \leq c_{0} R^{-1}$ when $r \geq c_{0}$ and $R \geq c_{0}$. This understood, it follows from (2-93), (2-96) with (2-5)-(2-6) from [14] that

$$
\left\langle\Delta^{\perp} \xi^{C \perp}, \eta^{\prime}\right\rangle_{\mathcal{L}^{2}} \geq c_{0}{ }^{-1}\left\|\eta^{C}\right\|_{\mathcal{L}^{2}}^{2}-c_{0} \delta\left\|\eta^{\prime}\right\|_{\mathcal{L}^{2}}^{2}
$$

when $r \geq c_{0}$ and $R \geq c_{0}$.

Fix $(\gamma, m) \in \Theta$, consider the term $\eta^{\gamma-}$ in the $\eta^{\prime}$ analog of (2-87). This $\eta^{\gamma-} \in \mathcal{L}_{1}^{2}$ had $\gamma$ - component $\eta_{\gamma-}^{\gamma-}$. By virtue of the fact that (2-73) has trivial cokernel, there is $\xi_{\gamma-\epsilon}$ $L_{1}^{2}\left(\mathbb{R} \times S^{1} ; \mathfrak{c}_{\gamma-} T^{1,0} \mathfrak{C}_{m}\right)$ that is mapped to $\eta_{\gamma-}^{\gamma-}$ by the operator in (2-73). Note that (2-93) holds when $C$ is replaced by $\gamma-$ in all appearances. Define an element $\xi^{\gamma-} \in \mathcal{L}_{1}^{2}$ as follows: Write its components as $\left(\left(\xi_{C}^{\gamma-}\right)_{C \in \mathcal{C}},\left(\xi_{\gamma^{\prime}-}^{\gamma-}\right)_{\left(\gamma^{\prime}, m\right) \in \Theta},\left(\xi_{\gamma^{\prime}+}^{\gamma-}\right)_{\left(\gamma^{\prime}, m\right) \in \Theta^{\prime}}\right)$. All components are zero except for $\xi_{\gamma-}^{\gamma-}$ and $\xi_{C}^{\gamma-}$ for those $C \in \mathcal{C}$ that intersect $U_{\gamma-\text {. }}$ The component $\xi_{\gamma-}^{\gamma-}$ is set equal to $\chi_{R-}^{\prime} \xi_{\gamma-}$ where $\chi_{R-}^{\prime}(w)=\chi((2.5 R+w) / R)$. Meanwhile, $\xi_{C}^{\gamma-}$ is defined from $\xi_{\gamma-}^{\gamma-}$ using the homomorphisms in (5-12) of [14]. Let $\xi^{\gamma-\perp}$ denote the $L^{2}$ orthogonal projection of $\xi^{\gamma-}$ to the complement of the image of $\phi_{0}$. An argument much like that used to derive (2-96) finds that

$$
\left\langle\Delta^{\perp} \xi^{\gamma-\perp}, \eta^{\prime}\right\rangle_{L^{2}} \geq c_{0}{ }^{-1}\left\|\eta^{\gamma-}\right\|_{\mathcal{L}^{2}}^{2}-c_{0} \delta\left\|\eta^{\prime}\right\|_{\mathcal{L}^{2}}^{2}
$$

when $r \geq c_{0}$ and $R \geq c_{0}$. Note that (2-97) uses the fact that $\chi_{R-}=0$ where $d \chi_{R_{-}}^{\prime} \neq 0$. Here, $\chi_{R-}$ is the cut-off function $\chi((3 R+w) / R)$ defined just prior to (5-7) in [14]. Each $(\gamma, m) \in \Theta^{\prime}$ has an analogous $\xi^{\gamma+\perp}$ that obeys the $\gamma+$ version of (2-97).

Let

$$
\xi=\sum_{C \in \mathcal{C}} \xi^{C \perp}+\sum_{(\gamma, m) \in \Theta} \xi^{\gamma-\perp}+\sum_{(\gamma, m) \in \Theta^{\prime}} \xi^{\gamma+\perp}
$$


Summing (2-96) and the various versions of (2-97) finds that

$$
\begin{aligned}
& \left\langle\Delta^{\perp} \xi, \eta^{\prime}\right\rangle_{\mathcal{L}^{2}} \\
& \geq c_{0}{ }^{-1}\left(\sum_{C \in \mathcal{C}}\left\|\eta^{C}\right\|_{\mathcal{L}^{2}}^{2}+\sum_{(\gamma, m) \in \Theta}\left\|\eta^{\gamma-}\right\|_{\mathcal{L}^{2}}^{2}+\sum_{(\gamma, m) \in \Theta}\left\|\eta^{\gamma+}\right\|_{\mathcal{L}^{2}}^{2}\right) \\
& -c_{0} \delta\left\|\eta^{\prime}\right\|_{\mathcal{L}^{2}}^{2}
\end{aligned}
$$

when $r \geq c_{0}$ and $R \geq c_{0}$. Given that the left hand side of (2-98) is zero, this inequality requires that each $C \in \mathcal{C}$ version of $\eta^{C}$, each $(\gamma, m) \in \Theta$ version of $\eta^{\gamma-}$ and each $(\gamma, m) \in \Theta^{\prime}$ version of $\eta^{\gamma+}$ have $L^{2}$ norm bounded by $c_{0} \delta\left\|\eta^{\prime}\right\|_{\mathcal{L}^{2}}$. What with the triangle inequality, this requires $\eta^{\prime}=0$ when $r$ and $R$ are large.

Part 7 It is a consequence of what is said in Lemma 2.7 and Lemma 2.8 that the index of $\mathfrak{D}_{E}$ is equal to that of $\Delta$, and the latter is equal to $\operatorname{dim}\left(V_{0}\right)-\operatorname{dim}\left(V_{1}\right)$. What follows derives a formula for this number.

To start, fix $C \in \mathcal{C}$ so as to consider the contribution to $\operatorname{dim}\left(V_{0}\right)-\operatorname{dim}\left(V_{1}\right)$ from $\operatorname{dim}\left(V_{0 C}\right)-\operatorname{dim}\left(V_{1 C}\right)$. A formula for this is obtained from Theorem 5.1 of [6]. Summing the results for the various elements in $\mathcal{C}$ gives

$$
\begin{aligned}
\sum_{C \in \mathcal{C}}\left(\operatorname{dim}\left(V_{0 C}\right)-\operatorname{dim}\left(V_{1 C}\right)\right) & \\
= & -\sum_{(\gamma, m) \in \Theta} m z_{\gamma, 1}+\sum_{(\gamma, m) \in \Theta^{\prime}} m z_{\gamma, 1}+\chi^{\Sigma}+2 \operatorname{deg}\left(N_{\Sigma}\right),
\end{aligned}
$$

where the notation is as follows: First, $z_{\gamma, 1}$ is the rotation number for $\gamma$ if $\gamma$ is hyperbolic. If $\gamma$ is elliptic with rotation number $\mathrm{R}$, then $z_{\gamma, 1}$ is one plus twice the greatest integer less than R. Second, $\chi^{\Sigma}$ denotes the Euler characteristic of $\Sigma$. Finally, $\operatorname{deg}\left(N_{\Sigma}\right)$ denotes the degree of the normal bundle of $\Sigma$ as defined using the section whose restriction to any given end is the real part of $\partial / \partial z$. It proves useful to invoke Lemma 2.3 to rid the formula of $\chi^{\Sigma}$. Doing so writes (2-99) as

$$
\begin{aligned}
& \sum_{C \in \mathcal{C}}\left(\operatorname{dim}\left(V_{0 C}\right)-\operatorname{dim}\left(V_{1 C}\right)\right) \\
&=-\sum_{(\gamma, m) \in \Theta} m z_{\gamma, 1}+\sum_{(\gamma, m) \in \Theta^{\prime}} m z_{\gamma, 1}-\left\langle c_{1}, \Sigma\right\rangle+\operatorname{deg}\left(N_{\Sigma}\right)-2 k_{\Sigma} .
\end{aligned}
$$

Fix $(\gamma, m) \in \Theta$ so as to consider the contribution to $\operatorname{dim}\left(V_{0}\right)-\operatorname{dim}\left(V_{1}\right)$ from $\operatorname{dim}\left(V_{0 \gamma-}\right)$. As a consequence of what is said in Part 3, this is zero if $m=1$. If $m>1$, then $\operatorname{dim}\left(V_{0 \gamma_{-}}\right)$is the sum of the $L^{2}$ indices of all of the $q \in\{1, \ldots, m\}$ versions of what is written in (2-75). Fix such an integer $q$. It is a consequence of (2-76) that the $L^{2}$ index 
of (2-75) is equal to twice the number of integers $n$ that obey

$$
q \mathrm{R}<n<q \mathrm{R}+(q-1)\left(k_{\mathrm{R}}^{>}-\mathrm{R}\right) .
$$

To put this in a different light, introduce $k_{q_{\mathrm{R}}}^{>}$for $q \geq 1$ to denote the least integer that is greater than $q \mathrm{R}$. Write $n=k_{q \mathrm{R}}^{>}+n^{\prime}$. Then $n^{\prime}$ must obey

$$
0 \leq n^{\prime}<q k_{\mathrm{R}}^{>}-k_{q \mathrm{R}}^{>} .
$$

Thus, the index is twice $q k_{\mathrm{R}}^{>}-k_{q \mathrm{R}}^{>}$. This understood,

$$
\operatorname{dim}\left(V_{0 \gamma-}\right)=2 \sum_{1 \leq q \leq m}\left(q k_{\mathrm{R}}^{>}-k_{q \mathrm{R}}^{>}\right) .
$$

Meanwhile, the analog of (2-101) for $(\gamma, m) \in \Theta^{\prime}$ leads to

$$
\operatorname{dim}\left(V_{0 \gamma+}\right)=2 \sum_{1 \leq q \leq m}\left(k_{q \mathrm{R}}^{<}-q k_{\mathrm{R}}^{<}\right),
$$

where $k_{q \mathrm{R}}^{<}$denotes the greatest integer that is less than $q R$.

Fix $(\chi, m)$ in either $\Theta_{-}$or $\Theta_{+}$. If $\gamma$ is elliptic, define $z_{\gamma, q}$ for $q \geq 1$ by $z_{\gamma, q}=2 k_{q \mathrm{R}}^{<}+1$. With this notation set, then (2-100), (2-103) and (2-104) sum to give

$$
\begin{aligned}
\operatorname{dim}\left(V_{0}\right)-\operatorname{dim}\left(V_{1}\right)=- & \left\langle c_{1}, \Sigma\right\rangle+\operatorname{deg}\left(N_{\Sigma}\right)-2 k_{\Sigma} \\
& -\sum_{(\gamma, m) \in \Theta} \sum_{1 \leq q \leq m} z_{\gamma, q}+\sum_{(\gamma, m) \in \Theta^{\prime}} \sum_{1 \leq q \leq m} z_{\gamma, q} \\
& +\sum_{(\gamma, m) \in \Theta} m(m-1) k_{\mathrm{R}}^{>}-\sum_{(\gamma, m) \in \Theta^{\prime}} m(m-1) k_{R}^{<} .
\end{aligned}
$$

Part 8 To put the right hand side of (2-105) in recognizable form, note that $\Sigma$ is not a surface that can be used to compute the $\Theta_{-}$and $\Theta_{+}$version of what Hutchings denotes as $I\left(\Theta_{-}, \Theta_{+}, \cdot\right)$, this the integer given in (1-4). This is because the ends of $\Sigma$ do not approach their limit Reeb orbits in the required fashion. A surface $Z$ for use in (1-4) can be obtained from $\Sigma$ as follows: First, $Z=\Sigma$ except where $|s| \gg R$. As it turns out, it is also permissable to take $Z=\Sigma$ in any $(\gamma, 1)$ version of either $U_{\gamma-}$ or $U_{\gamma+}$. However, such is not the case for a $(\gamma, m)$ version with $m>1$.

What follows describes $Z$ in a given $(\gamma, m) \in \Theta_{-}$version of $U_{\gamma-}$ when $m>1$. Let $\mathcal{E}$ denote an end of $\Sigma$ that intersects $U_{\gamma-}$. As constructed, $\mathcal{E}$ is the graph of the function $(w, t) \rightarrow z(w, t)=z_{\mathcal{E}} e^{-\mathrm{R} w} e^{k_{\mathrm{R}}^{>}(w+i t)}$ where $z_{\mathcal{E}}$ is an $m$-th root of unity and $\mathrm{R}$ is the rotation number. Now identify $\mathbb{R} \times S^{1}$ with $\mathbb{C}^{*}$ using the function 
$(w, t) \rightarrow x=e^{(w+i t)}$. Then $\Sigma$ intersects $U_{\gamma-} \subset \mathbb{C}^{*} \times \mathbb{C}$ as the locus of points $(x, z)$ where

$$
x^{m k_{\mathrm{R}}^{>}} e^{-m \mathrm{R} w}-z^{m}=0 .
$$

The model for $Z$ 's intersection with the $w \ll-1$ part of $U_{\gamma-}$ is the locus where

$$
x^{m k_{\mathrm{R}}^{>}} e^{-m \mathrm{R} w}-z^{m}=\chi_{R-\varepsilon}
$$

with $\varepsilon>0$ but much less than 1 . Here again, $\chi_{R_{-}}(w)=\chi((3 R+w) / R)$. The latter is a smooth curve for small $\varepsilon$. It has $m$ ends that have the required asymptotics for use in computing $I\left(\Theta_{-}, \Theta_{+}, \cdot\right)$. Let $\mathfrak{s}$ denote the section of the normal bundle of $Z$ in $U_{\gamma-}$ that is defined by the normal projection of the vector field $\partial / \partial z$. As can be seen readily by comparing (2-107) with (2-106), if $\varepsilon$ is sufficiently small, then this section has precisely $m(m-1) k_{\mathrm{R}}^{>}$extra zeros on $Z$ 's intersection with $U_{\gamma-}$, and each such zero counts with weight 1 .

A similar construction can be made to obtain $Z$ in any $(\gamma, m) \in \Theta_{+}$version of $U_{\gamma+}$. In this case, the section of $Z$ 's normal bundle defined by $\partial / \partial z$ in $U_{\gamma+}$ has $m(m-1) k_{\mathrm{R}}^{<}$ zeros with each having sign -1 .

Granted the preceding observations, it follows that what is denoted by $Q_{z}$ in (1-4) is given by

$$
Q_{z}=\operatorname{deg}\left(N_{\Sigma}\right)+\sum_{(\gamma, m) \in \Theta} m(m-1) k_{\mathrm{R}}^{>}-\sum_{(\gamma, m) \in \Theta^{\prime}} m(m-l) k_{\mathrm{R}}^{<} .
$$

Substitute (2-108) into (2-105). A comparison with (1-4) then finds

$$
\operatorname{dim}\left(V_{0}\right)-\operatorname{dim}\left(V_{1}\right)=I\left(\Theta_{-}, \Theta_{+} ; Z\right)-2 k_{\Sigma} .
$$

The formula in Theorem 1.1 for $\operatorname{deg}_{\mathrm{SW}}\left(\Phi^{r}\left(\chi_{+}\right)\right)-\operatorname{deg}_{\mathrm{SW}}\left(\Phi^{r}\left(\chi_{-}\right)\right)$when (2-55) holds for each Reeb orbit from either $\Theta_{-}$or $\Theta_{+}$follows from (2-109), Lemma 2.8, what is said after Lemma 2.7, and Lemma 2.5.

\section{2.d The relative degree formula: Part III}

Suppose that $\Theta_{-}$and $\Theta_{+}$are elements in $Z$, but assume no longer that their Reeb orbits obey (2-55). Fix $x_{-} \in \mathfrak{C} \Theta_{-}^{*}$ and $x_{+} \in \mathfrak{C}_{+}^{*}$. This subsection completes the proof of Theorem 1.1 by verifying its formula for $\operatorname{deg}_{\mathrm{SW}}\left(\Phi^{r}\left(\chi_{+}\right)\right)-\operatorname{deg}\left(\Phi^{r}\left(\chi_{-}\right)\right)$. This is done in three parts. 
Part 1 To start, take $L>\Sigma_{(\gamma, m) \in \Theta_{-}} m \ell_{\gamma}+\Sigma_{(\gamma, m) \in \Theta_{+}} m \ell_{\gamma}$ and $\delta>0$ but very small. Proposition 2.5 in [13] asserts that there is a $(\delta, L)$ approximation to $(a, J)$. In particular, pair $(\hat{a}, \widehat{J})$ of contact 1 -form and almost complex structure in $\mathcal{J}_{\widehat{a}}$ has the following properties:

- There exists a smoothly parametrized family of pairs $\left\{\left(a_{\tau}, J_{\tau}\right)\right\}_{\tau \in[0,1]}$ of contact 1-form and almost complex structure in $J_{a_{\tau}}$ such that the $\tau=0$ version is $(a, J)$ and the $\tau=1$ version is $(\hat{a}, \widehat{J})$.

- Suppose that $\gamma$ is a Reeb orbit for $a$ 's Reeb vector field with $\ell_{\gamma}<L$. For each $\tau \in[0,1]$, the loop $\gamma$ is a Reeb orbit for $a_{\tau}$ 's Reeb vector field. Moreover, if $\gamma$ is hyperbolic for $a$, then $\gamma$ is hyperbolic for $a_{\tau}$, and if it is elliptic for $a$, then it is elliptic for $a_{\tau}$. In addition, the $a$ and $a_{\tau}$ versions of $\ell_{\gamma}$ are equal. Finally, there is fixed tubular neighborhood (2-110) $\quad \operatorname{map} \varphi: S^{1} \times D \rightarrow M$ for $\gamma$ that pulls back $a_{\tau}$ as in (1-1) with $(\nu, \mu)$ now functions of $\tau$. With respect to this map, the rotation number of $\gamma$ is independent of $\tau \in[0,1]$.

- Suppose again that $\gamma$ is a Reeb orbit for $a$ 's Reeb vector field with $\ell_{\gamma}<L$. Let $\varphi$ denote the tubular neighborhood map just described. If $\gamma$ is hyperbolic with rotation number $k$, then the $\hat{a}$ version of the pair $(\nu, \mu)$ is $\left(\frac{1}{4} k, i \varepsilon e^{i k t}\right)$ with $\varepsilon \in(0, \delta)$. If $\gamma$ is elliptic with rotation number $\mathrm{R}$, then the $\hat{a}$ version of $(\nu, \mu)$ is $\left(\frac{1}{2} \mathrm{R}, 0\right)$.

This pair $(\hat{a}, \hat{J})$ is sufficient for what follows when there are no pairs $(\gamma, m)$ in either $\Theta_{-}$or $\Theta_{+}$with $\gamma$ hyperbolic and $m>1$. If the latter condition is not satisfied, then an additional homotopy must be made in a small tubular neighborhood of the Reeb orbit from any pair of the sort where the latter condition is violated. This said, let $\gamma$ denote such a hyperbolic Reeb orbit that is paired with $m>1$ in either $\Theta_{-}$or $\Theta_{+}$. Let $k$ denote its rotation number. Fix a small irrational number $\mathrm{R}^{\prime} \in(0, \varepsilon)$ and consider the homotopy $\left\{\hat{a}_{\tau}\right\}_{\tau \in[0,1]}$ that replaces the top line in (1-1) with

$$
\begin{aligned}
\frac{2 \pi}{\ell_{\gamma}} \varphi^{*} \hat{a}_{\tau}=\left(1-\frac{1}{2}\left(\frac{1}{2} k-\tau \mathrm{R}^{\prime}\right)|z|^{2}\right. & \\
& \left.-(1-\tau) i \varepsilon\left(e^{i k t} \bar{z}^{2}-e^{-i k t} z^{2}\right) d t\right)+\frac{i}{2}(z d \bar{z}-\bar{z} d z)
\end{aligned}
$$

where $|z| \leq \frac{1}{4} \varepsilon$, and leaves the top line unchanged where $|z|>\frac{1}{2} \varepsilon$. Such a homotopy has the following property: The loop $\gamma$ is a Reeb orbit for all $\hat{a}_{\tau}$. However, it is elliptic with rotation number $\frac{1}{2} k-\mathrm{R}^{\prime}$ with respect to $\hat{a}_{\tau=1}$. Note that the homotopy $\tau \rightarrow \hat{a}_{\tau}$ 
can be constructed so that

$$
\left|\nabla^{\otimes p}\left(\hat{a}_{\tau}-\widehat{a}\right)\right| \leq c_{0} \varepsilon^{3-p} \quad \text { for } p \in\{0,1,2\}
$$

Each contact form $\hat{a}_{\tau}$ has an associated compatible almost complex structure that differs from $\hat{J}$ only on the radius $\varepsilon$ neighborhoods of the hyperbolic Reeb orbits that are paired either in $\Theta_{-}$or $\Theta_{+}$with an integer greater than 2. A smooth family $\left\{\widehat{J}_{\tau}\right\}_{\tau \in[0,1]}$ of such almost complex structures can be chosen so that $\left(\widehat{a}_{1}, \widehat{J}_{1}\right)$ obeys the $\mathrm{R}=\frac{1}{2} k-\mathrm{R}^{\prime}$ version of (1-14), and so that $\widehat{J}_{1} \in \mathcal{J}_{\widehat{a}_{1}}$. One can assume, in addition, that this family is such that

$$
\left|\nabla^{\otimes p}\left(\widehat{J}_{\tau}-\widehat{J}\right)\right| \leq c_{0} \varepsilon^{2-p} \quad \text { for } p \in\{0,1,2\} .
$$

Use $\Theta_{-}^{\prime}$ to denote the same set as $\Theta_{-}$, but now viewed as a collection of pairs of the form $(\gamma, m)$ as defined using the contact form $\widehat{a}_{\tau=1}$ and the almost complex structure $\widehat{J}_{1}$. Define $\Theta_{+}^{\prime}$ from $\Theta$ in the same fashion.

Part 2 Both $\Theta_{-}^{\prime}$ and $\Theta_{+}^{\prime}$ are described by (2-65). According to what is said in Lemmas 2.1-2.4 in [14], both $\mathfrak{C} \Theta_{-}^{\prime *}$ and $\mathfrak{C}_{+}^{\prime *}$ contain a single element; these elements are denoted respectively by $\chi_{-}^{\prime}$ and $\chi_{+}^{\prime}$.

If $r \geq c_{0}$, there is a corresponding $\left(\widehat{a}_{1}, \widehat{J}_{1}\right)$ versions of $\Phi^{r}$ that assign to $\chi_{-}^{\prime}$ and $\chi_{+}^{\prime}$ solutions to corresponding version of (1-8). Let $\mathfrak{c}_{-}^{\prime}$ and $\mathfrak{c}_{+}^{\prime}$ denote the solutions to these respective versions of (1-8). Both $\mathfrak{c}_{-}^{\prime}$ and $\mathfrak{c}_{+}^{\prime}$ are pairs in $\operatorname{Conn}(E) \times C^{\infty}\left(M ; \mathbb{S}^{\prime}\right)$ where $\mathbb{S}^{\prime}$ is the spinor bundle for the metric that is associated to $\left(\widehat{a}_{1}, \widehat{J}_{1}\right)$. Both $\mathfrak{c}_{-}^{\prime}$ and $\mathfrak{c}_{+}^{\prime}$ have corresponding versions of the operator $\mathfrak{L}_{\mathfrak{c}}$ as depicted in (1-9); and both versions have trivial kernel when $r$ is large. As a consequence, there is a corresponding spectral flow from the $\mathfrak{c}_{-}^{\prime}$ version of $\mathfrak{L}_{\mathfrak{c}}$ to the $\mathfrak{c}_{+}^{\prime}$ version.

As argued in the previous two subsections the spectral flow from the $\mathfrak{c}_{-}^{\prime}$ version of $\mathfrak{L}_{\mathfrak{c}}$ to the $\mathfrak{c}_{+}^{\prime}$ version is equal to $\operatorname{deg}_{\text {ech }}\left(x_{+}^{\prime}\right)-\operatorname{deg}_{\text {ech }}\left(x_{-}^{\prime}\right) \bmod (p)$. Note in addition, that if $\mathrm{R}^{\prime}$ is sufficiently small, then $\operatorname{deg}_{\text {ech }}\left(\chi_{+}^{\prime}\right)-\operatorname{deg}_{\text {ech }}\left(\chi_{-}^{\prime}\right)=\operatorname{deg}_{\text {ech }}\left(\chi_{+}\right)-\operatorname{deg}_{\text {ech }}\left(\chi_{-}\right)$. Indeed, this follows from (1-4) given the definition of the Conley-Zehnder indices in Step 3 of Part 2 in Section 1.a.

Part 3 Let $\Theta$ and $\Theta^{\prime}$ now denote either $\Theta_{-}$and $\Theta_{-}^{\prime}$ or $\Theta_{+}$and $\Theta_{+}^{\prime}$. Use $\chi$ and $x^{\prime}$ to denote the corresponding $x_{-}$and $x_{-}^{\prime}$ or $x_{+}$and $x_{+}^{\prime}$. Now consider the homotopy from the original pair $(a, J)$ to $\left(\widehat{a}_{1}, \widehat{J}_{1}\right)$ to the original pair. Reparametrize this homotopy by $\tau \in[0,1]$. Given that $\Theta$ and $\Theta^{\prime}$ contain the same Reeb orbits with the same integer partners, such a homotopy can be covered by a corresponding homotopy that changes the data $\chi$ to $\chi^{\prime}$. Note in this regard that $\chi^{\prime}$ is such that each $(\gamma, m)$ version of $\mathfrak{c}_{\gamma}$ is the symmetric vortex in $\mathfrak{C}_{m}$. Let $\left\{\chi_{\tau}\right\}_{\tau \in[0,1]}$ denote this 
covering homotopy. The constructions in Section 3.a of [14] can be done for each $\chi_{\tau}$. This with each $(\gamma, m) \in \Theta$ version of $\xi_{\gamma}=0$, produce a configuration, $\left(A^{* \tau}, \psi^{* \tau}\right)$, in $\operatorname{Conn}(E) \times C^{\infty}\left(M ; \mathbb{S}_{\tau}\right)$. Here, $\mathbb{S}_{\tau}$ is the spinor bundle that is defined using the metric that is defined by $\tau$ 's version of the contact form and almost complex structure. Each $x_{\tau}$ has a version of what is denoted in Step 2 of Section 2.a as $\left(b_{*}, \eta_{*}\right)$, this denoted here as $\left(b_{* \tau}, \eta_{* \tau}\right)$. Use $\mathfrak{L}_{* \tau}$ to denote the version of (1-9) that is defined by $\left(A^{\tau}, \psi^{\tau}\right)=\left(A^{* \tau}+2^{1 / 2} r^{1 / 2} b_{* \tau}, \psi^{* \tau}+2^{1 / 2} r^{1 / 2} \eta_{* \tau}\right)$. It follows from what is said in Steps 8 and 9 of Section 2.a as applied to $\chi$ and to $\chi^{\prime}$ that the spectral flow of the family $\left\{\mathfrak{L}_{* \tau}\right\}_{\tau \in[0,1]}$ is the same as that from the $\mathfrak{c}=\mathfrak{c}(\chi)$ version of $\mathfrak{L}_{\mathfrak{c}}$ to the $\mathfrak{c}=\mathfrak{c}\left(x^{\prime}\right)$ version.

To compute the latter spectral flow, remark that there is a version of (2-19) for each $\mathfrak{L}_{* \tau}$, with corresponding terms $\wp_{\tau}, \mathfrak{L}_{\tau}^{\perp}$ and $\mathrm{D}_{\tau}$. The bounds in (2-20)-(2-22) hold in each case. Meanwhile, if $(\gamma, m) \in \Theta$, then

$$
\left(\mathrm{D}_{\tau} \theta\right)_{\gamma}=\frac{i}{2} \nabla_{t} \theta_{\gamma}+\left.\left(\nabla_{\left(\theta_{\gamma}\right)_{\mathbb{R}}} \nabla^{1,0} \hat{h}_{\tau}\right)\right|_{\mathfrak{c}_{\gamma}^{\tau}}+\mathfrak{e}_{\gamma},
$$

where $\mathfrak{c}_{\gamma}^{\tau}$ is the map from $S^{1} \rightarrow \mathfrak{C}_{m}$ that is defined by $\chi_{\tau}$. Here, $\left\|\mathfrak{e}_{\gamma}\right\|_{L^{2}} \leq c_{0}\|\theta\|_{L_{1}^{2}}$. The proof is identical to that used to prove the formula for $(\mathrm{D} \theta)_{\gamma}$ given in Lemma 2.1. What with the $\mathfrak{L}_{* \tau}$ versions of (2-20)-(2-22), these formulae for $\left\{\mathrm{D}_{\tau}\right\}_{\tau \in[0.1]}$ imply that the spectral flow for the family $\left\{\mathfrak{L}_{* \tau}\right\}_{\tau \in[0,1]}$ is the sum of the spectral flows for the various $(\gamma, m) \in \Theta$ versions of $\left\{(i / 2) \nabla_{t} \theta_{\gamma}+\left.\left(\nabla_{\left(\theta_{\gamma}\right)} \nabla_{\mathbb{R}} \nabla^{1,0} f_{\tau}\right)\right|_{\mathfrak{c}_{\gamma}^{\tau}}\right\}_{\tau \in[0,1]}$.

This last conclusion with what is said at the end of Part 2 imply the formula asserted by Theorem 1.1.

\section{Proof of Theorem 1.2}

This section proves the assertions of Theorem 1.2 about the map $\Psi^{r}$. Section 3.a proves the assertion that is made by the first bullet. Section 3.b proves the assertion that is made by the second bullet.

\section{3.a Nondegeneracy}

Fix $L \geq 1$ and a pair $\Theta_{-}$and $\Theta_{+}$in $Z_{\text {ech }}^{L}$. Suppose that $\Sigma$ obeys the $\left(\Theta_{-}, \Theta_{+}\right)$ versions of the constraints in Section 4.b of [14]. This being the case, the constructions in Sections 4-7 of [14] produce a family of instanton solutions to (1-11) that are parametrized by a small radius ball about the origin in the vector space $V_{0}$ of Proposition 7.1 in [14]. Let $\mathfrak{d}_{\Sigma}: \mathbb{R} \rightarrow \operatorname{Conn}(E) \times C^{\infty}(M ; \mathbb{S})$ denote such an instanton. Use $\mathfrak{c}_{-}$and $\mathfrak{c}_{+}$to denote the respective $s \rightarrow-\infty$ and $s \rightarrow \infty$ limits is $\mathfrak{d}_{\Sigma}$. Let $\mathfrak{D}$ denote 
the corresponding version of the operator that is depicted in (1-12) The upcoming Proposition 3.1 describes this operator.

Proposition 3.1 refers to an integer $I\left(\Theta_{-}, \Theta_{+} ; Z\right)$. The latter is defined by (1-4) using for $Z$ a suitable embedded, oriented surface in $[0,1] \times M$ with the following property: The respective closures of the projections to $M$ of $Z$ and $\Sigma_{(C, m) \in \Sigma} m C$ are homologous rel their boundaries. The proposition also refers to a parameter $R$. This is the parameter introduced in Section 4.c of [14] and used subsequently in the construction of the map $\Psi^{r}$.

Proposition 3.1 There exists $\kappa>1$ with the following significance: Fix $R \geq \kappa^{2}$ and $r \geq \kappa$. Then Propositions 6.4, 7.1 and 7.6 of [14] can be invoked to construct an instanton solution to (1-11) from any element in the radius $\kappa^{-1}$ ball about the origin in the vector space $V_{0}$. Fix such a point to define the instanton $\mathfrak{d}_{\Sigma}$ and the corresponding operator $\mathfrak{D}$ maps $L_{1}^{2}\left(\mathbb{R} \times M ; i T^{*} M \oplus \mathbb{S} \oplus i \mathbb{R}\right)$ to $L^{2}\left(\mathbb{R} \times M ; i T^{*} M \oplus \mathbb{S} \oplus i \mathbb{R}\right)$ as a Fredholm operator. This operator has trivial cokernel and index equal to $I\left(\Theta_{-}, \Theta_{+} ; Z\right)$.

This proposition is proved momentarily.

Proposition 3.1 implies directly what is asserted by the first item of the first bullet in Theorem 1.2. The second item follows from the first given the fact that $\Psi^{r}$ was defined so as to be equivariant with respect to the respective $\mathbb{R}$ actions on $\mathcal{M}_{1}\left(\Theta_{-}, \Theta_{+}\right)$and on $\mathcal{M}_{1}\left(\mathfrak{c}_{-}, \mathfrak{c}_{+}\right)$.

Proof of Proposition 3.1 The fact that $\mathfrak{D}$ is Fredholm follows directly from Theorem 1.1 's assertion that both $\mathfrak{c}_{-}$and $\mathfrak{c}_{+}$are nondegenerate. If $c_{1}(\operatorname{det}(\mathbb{S}))$ is torsion, then the fact that $\mathfrak{D}$ has the asserted index follows directly from formula given in Theorem 1.1 for the $\operatorname{deg}_{\mathrm{SW}}\left(\mathfrak{c}_{+}\right)-\operatorname{deg}_{\mathrm{SW}}\left(\mathfrak{c}_{-}\right)$. The computation of its index in the other cases follows from the arguments given below that establish the dimension of its kernel and the trivial nature of its cokernel. These arguments constitute Parts 1-6 of what follows.

Part 1 Reintroduce the pair $\left(A^{*}, \psi^{*}\right)$ as defined in Section 5.a of [14]. Let $\mathcal{B} \subset \mathcal{K}$, $V_{0}$ and the map $q: \mathcal{B} \rightarrow V_{0}$ be as described in Proposition 7.1 of [14]. Let $\lambda \in q(\mathcal{B})$ denote the element that defines the instanton $\mathfrak{d}_{\Sigma}$. Introduce $\xi \in \mathcal{B} \cap q^{-1}(\lambda)$ to denote the element that is subsequently supplied by that same proposition. Section 5.b of [14] describes $\left(A^{\xi}, \psi^{\xi}\right)$. Section 6.d of [14] defines $\mathfrak{h}=\mathfrak{h}(\xi)$ and Proposition 6.4 of [14] describes $\mathfrak{q}=\mathfrak{q}(\xi) \in \mathbb{H}_{\xi}^{\perp}$. As noted in Proposition 7.6 of [14], the instanton $\mathfrak{d}_{\Sigma}$ is obtained from the $(\xi, \mathfrak{b}=\mathfrak{h}+\mathfrak{q})$ version of the pair $(A, \psi)$ given in (5-19) of [14] by acting on the latter with a suitable map from $\mathbb{R} \times M$ to $S^{1}$. In any event, agree now to use $\left(A_{\Sigma}, \psi_{\Sigma}\right)$ to denote this same pair $(A, \psi)$. 
Set $X=\mathbb{R} \times M$. With its product metric defining the Hodge star and the notions of selfand anti-self-duality, define the operator $\mathfrak{D}_{\Sigma}$ by replacing $\left(A_{X}, \psi_{X}\right)$ by $\left(A_{\Sigma}, \psi_{\Sigma}\right)$ in (2-61). This operator is the gauge transform of the operator $\mathfrak{D}$ via the map from $\mathbb{R} \times M$ that writes the instanton $\mathfrak{d}_{\Sigma}$ as $\left(A_{\Sigma}, \psi_{\Sigma}\right)$. Note in this regard that if $X=\mathbb{R} \times M$ and the metric is the product metric, then the bundle $i T^{*} X \oplus \mathbb{S}_{E+}$ whose sections define the domain space in (2-61), and the bundle $i \Lambda^{2+} \oplus \mathbb{S}_{E-} \oplus i \mathbb{R}$ whose sections defines the range space are both canonically isomorphic to $i T^{*} M \oplus \mathbb{S} \oplus i \mathbb{R}$, which is the domain and range space of $\mathfrak{D}$. These identifications of domain and range spaces are implicit in what follows.

The plan for what follows is to compare $\mathfrak{D}_{\Sigma}$ with an operator, $\mathfrak{D}_{\xi}$, that is more accessible. To define $\mathfrak{D}_{\xi}$, introduce the connection $\left(A^{\xi}, \psi^{\xi}\right)$ from (5-15) in [14] and introduce $\mathfrak{h}=\mathfrak{h}(\xi)$ from Section 6.d and Proposition 6.4 of [14] and write its components as $\left(b_{\mathfrak{h}}, \eta_{\mathfrak{h}}, \phi_{\mathfrak{h}}\right)$. Now define $\mathfrak{D}_{\xi}$ by (2-61) using $X=\mathbb{R} \times M$ and using for $\left(A_{X}, \psi_{X}\right)$ the pair $\left(A^{\xi}+(2 r)^{1 / 2}\left(\phi_{\mathfrak{h}} d s+b_{\mathfrak{h}}\right), \psi^{\xi}+\eta_{\mathfrak{h}}\right)$. The comparison between $\mathfrak{D}_{\Sigma}$ and $\mathfrak{D}_{\xi}$ will be made by writing

$$
\mathfrak{D}_{\Sigma} \mathfrak{f}=\mathfrak{D}_{\xi} \mathfrak{f}+r^{1 / 2} \mathfrak{e}_{\mathfrak{q}}^{*} \mathfrak{f}
$$

where $\mathfrak{e}_{\mathfrak{q}}$ is the contribution from $\mathfrak{q}$. Note that $\left|\mathfrak{e}_{\mathfrak{q}}\right| \leq c_{0}|\mathfrak{q}|$. Meanwhile,

$$
\|\mathfrak{q}\|_{\mathbb{H}}+\|\mathfrak{q}\|_{*} \leq c_{0} r^{1 / 2}\left(r^{-1 / 2+8 \sigma}+|\lambda|\right) .
$$

These bound on $\mathfrak{q}$ comes via Propositions 7.1 and 6.4 in [14].

Part 2 It proves useful when discussing (3-1) to introduce the Hilbert space $\mathbb{H}$ as defined in (6-2) of [14]. Introduce $\mathcal{K}_{1}^{2}$ to denote the Hilbert space that is defined as in Step 4 of Section 5.b in [14], but with each norm $\|\cdot\|_{\mathcal{K}}$ in (5-13) of [14] replaced by the corresponding $L_{1}^{2}$ norm. (The latter is denoted by $\|\cdot\|_{\mathcal{K}_{1}^{2}}$ in what follows.) Use (6-9) in [14] to define the homomorphism $t_{\xi}: \mathcal{K}_{1}^{2} \rightarrow \mathbb{H}$. This $t_{\xi}$ is injective when $r \geq c_{0}$. Define $\mathbb{H}_{\xi} \frac{\perp}{\xi}$ as in Part 6 of Section 6.a in [14], this the $L^{2}$ orthogonal complement in $\mathbb{H}$ of the image of $t_{\xi}$. An element $\mathfrak{f} \in \mathbb{H}$ has the $L^{2}$-orthogonal decomposition $\mathfrak{f}=\mathfrak{f}^{\perp}+t_{\xi}(\theta)$ where $\mathfrak{f}^{\perp} \in \mathbb{H}_{\frac{\xi}{\xi}}^{\perp}$ and where $\theta \in \mathcal{K}_{1}^{2}$.

Use $\mathbb{L}$ to denote the space $L^{2}\left(\mathbb{R} \times M ; i T^{*} M \oplus \mathbb{S} \oplus i \mathbb{R}\right)$ and define $\mathcal{L}^{2}$ as in Part 7 of Section 6.a in [14]. The formulae in (6-9) of [14] define an injective map, this also denoted by $t_{\xi}$, from $\mathcal{L}^{2}$ to $\mathbb{L}$. Note that the large $r$ versions of $t_{\xi}: \mathcal{L}^{2} \rightarrow \mathbb{L}$ are such that

$$
c_{0}^{-1}\|\theta\|_{L^{2}} \leq\left\|t_{\xi}(\theta)\right\|_{2} \leq c_{0}\|\theta\|_{L^{2}} .
$$

In any event, both the $\mathcal{K}_{1}^{2}$ and $\mathcal{L}^{2}$ versions of $t_{\xi}$ have two properties to keep in mind for now. To describe them, introduce as in Section 5.a of [14], the symbols $\Xi_{\Sigma-}$ 
and $\Xi_{\Sigma+}$ to denote the respective sets of distinct Reeb orbits that are approached as $s \rightarrow-\infty$ and as $s \rightarrow+\infty$ by the constant $s$ slices of $\Sigma$. Thus, $\gamma \in \Xi_{\Sigma-}$ if and only if $\gamma=\gamma_{E}$ for some negative $s \ll-1$ end of $\Sigma$. When $\gamma \in \Xi_{\Sigma-}$, the symbol $\mathcal{E}_{\gamma-}$ is used to denote the set of ends $\mathcal{E} \in \mathcal{E}$ with $\gamma_{\mathcal{E}}=\gamma$. There is the analogous definition of $\mathcal{E}_{\gamma+}$ for each $\gamma \in \Xi_{\Sigma+}$.

Here is the first property: The set $\left(\bigcup_{C \in \mathcal{C}} U_{C}\right) \cup\left(\bigcup_{\gamma \in \Xi_{\Sigma-}} U_{\gamma-}\right) \cup\left(\bigcup_{\gamma \in \Xi_{\Sigma+}} U_{\gamma+}\right)$ contains the support of $t_{\xi}(\theta)$. To state the second property, write the element $\theta$ as $\left(\left(\theta_{C}\right)_{C \in \mathcal{C}},\left(\theta_{\gamma_{-}}\right)_{\gamma \in \Xi_{\Sigma-}},\left(\theta_{\gamma+}\right)_{\gamma \in \Xi_{\Sigma+}}\right)$. On any given $C \in \mathcal{C}$ version of $U_{C}$,

$$
\left|t_{\xi}(\theta)\right| \leq c_{0} r^{1 / 2} e^{-\sqrt{r} \operatorname{dist}(\cdot, \Sigma) / 100}\left|\theta_{C}\right| .
$$

Meanwhile, on any given version of $U_{\gamma-}$ or $U_{\gamma+}$, the analogous inequality holds with $\theta_{\gamma-}$ or $\theta_{\gamma}+$ replacing $\theta_{C}$.

Part 3 This part gives bounds for the $L^{2}$ norms of the endomorphism $r^{1 / 2} \mathfrak{e}_{\mathfrak{q}}^{*}(\cdot)$ that appears in (3-1). Consider its norm when acting on a given $\mathfrak{f}^{\perp} \in \mathbb{H} \frac{\perp}{\xi}$. The bounds in (3-2) imply directly that

$$
r^{1 / 2}\left\|\mathfrak{e}_{\mathfrak{q}}^{*} \mathfrak{f}^{\perp}\right\|_{2} \leq c_{0} r^{1 / 2}\|\mathfrak{q}\|_{\mathbb{H}}\left\|\mathfrak{f}^{\perp}\right\|_{\mathbb{H}} \leq c_{0}\left(r^{-1 / 2+8 \sigma}+|\lambda|\right)\left\|\mathfrak{f}^{\perp}\right\|_{\mathbb{H}} .
$$

Indeed, this follows by first bounding the $L^{2}$ norm of $|\mathfrak{q}||\mathfrak{f}|$ by the product of their $L^{4}$ norms, and then bounding the latter using (6-3) in [14].

Consider next the $L^{2}$ norm of $r^{1 / 2} \mathfrak{e}_{\mathfrak{q}}^{*} t_{\xi}(\theta)$ for $\theta \in \mathcal{L}_{1}^{2}$. To obtain a useful bound, write $\theta=\left(\left(\theta_{C}\right)_{C \in \mathcal{C}},\left(\theta_{\gamma-}\right)_{\gamma \in \Xi_{\Sigma-}},\left(\theta_{\gamma+}\right)_{\gamma \in \Xi_{\Sigma_{+}}}\right)$. Fix $C \in \mathcal{C}$. It follows from (3-4) that the contribution from $U_{C}$ to $r^{1 / 2}\left\|\mathfrak{e}_{\mathfrak{q}}^{*} t_{\xi}(\theta)\right\|_{2}$ is bounded by the square root of

$$
c_{0} \int_{C-\cup_{\mathcal{E} \in \mathcal{E}_{C}} \mathcal{E}_{2 R}}\left|\theta_{C}\right|^{2} f,
$$

where $f$ is the function on $C-\left(\bigcup_{\mathcal{E} \in \mathcal{E}_{C}} \mathcal{E}_{4 R}\right)$ given by

$$
f(\cdot)=r^{2} \int_{|\mathfrak{s}| \leq 2 \rho_{\Sigma}} e^{-\sqrt{r}|\mathfrak{s}| / c_{0}}|\mathfrak{q}|^{2} \chi_{4 \rho_{\Sigma}}
$$

Here, $\rho_{\Sigma}=r^{-1 / 2+\sigma}$ is from (4-8) in [14]. Meanwhile, $\chi_{T}$ for any given $T>0$ is shorthand for the function on the normal bundle $N \rightarrow C$ that given by $\chi(|\mathfrak{s}| / T)$. To bound (3-6), fix any given $\rho \in(0,1)$ and a disk $D_{\rho} \subset C-\left(\bigcup_{\mathcal{E} \in \mathcal{E}_{C}} \mathcal{E}_{4 R}\right)$ of radius $\rho$. As argued momentarily, there exists $c_{0}$ and $\delta>c_{0}{ }^{-1}$ such that

$$
\int_{D_{\rho}} f \leq c_{0}\left(r^{-1+16 \sigma}+|\lambda|^{2}\right) \rho^{\delta}
$$


Granted (3-8), use Lemma 5.4.1 in [14] to conclude that the integral in (3-6) is bounded by $c_{0}\left(r^{-1+16 \sigma}+|\lambda|^{2}\right)\|\theta\|_{\mathcal{K}_{1}^{2}}^{2}$. A very similar argument finds the same sort of bound for the square of the $L^{2}$ norm over any $U_{\gamma-}$ or $U_{\gamma+}$ of $r^{1 / 2}\left\|\mathfrak{e}_{\mathfrak{q}}^{*} t_{\xi}(\theta)\right\|_{2}$. Thus,

$$
r^{1 / 2}\left\|\mathfrak{e}_{\mathfrak{q}}^{*} t_{\xi}(\theta)\right\|_{2} \leq c_{0}\left(r^{-1 / 2+8 \sigma}+|\lambda|\right)\|\theta\|_{\mathcal{K}_{1}^{2}} .
$$

To obtain (3-8), consider first the case when $\rho \leq r^{-2}$. In this case, Hölder's inequality finds that the left hand side of (3-8) is bounded by $c_{0} r^{3 / 2} \rho\|\mathfrak{q}\|_{L^{4}}^{2}$. This is bounded in turn by $c_{0}(\rho r)^{1 / 2} r\|\mathfrak{q}\|_{\mathbb{H}}^{2}$ using the Sobolev inequality in (6-3) of [14]. According to Proposition 7.1 in [14], this is less than the $\delta=1 / 2$ version of what is written on the right hand side of (3-8).

Consider next the case where $\rho>r^{-2}$. This argument here exploits the identity

$$
s e^{-\sqrt{r} s / c_{0}}=-c_{0} r^{-1 / 2} \frac{d}{d s}\left(s e^{-\sqrt{r} s / c_{0}}+c_{0} r^{-1 / 2}\left(e^{-\sqrt{r} s / c_{0}}-1\right)\right) .
$$

This and integration by parts finds

$$
\begin{aligned}
f \leq c_{0} r^{3 / 2} \int_{|\mathfrak{s}| \leq 2 \rho_{\Sigma}}\left(\left(e^{-\sqrt{r}|\mathfrak{s}| / c_{0}}\right.\right. & \left.+r^{-1 / 2}|\mathfrak{s}|^{-1}\left(e^{-\sqrt{r}|s| / c_{0}}-1\right)\right)|\mathfrak{q} \| \nabla \mathfrak{q}| \\
& \left.+r^{-10} e^{-\sqrt{r}|s| / 2 c_{0}} \chi_{8 \rho_{\Sigma}}\left(1-\chi_{\rho_{\Sigma}}\right)|\mathfrak{q}|^{2}\right) .
\end{aligned}
$$

It then follows using Hölder's inequality that the integral of $f$ over the disk $D_{\rho}$ is bounded by $c_{0} r\|\mathfrak{q}\|_{\mathbb{H}}\left(\int_{\pi^{-1}\left(D_{\rho}\right)}|\nabla \mathfrak{q}|^{2}\right)^{1 / 2}+r^{-8}\|\mathfrak{q}\|_{\mathbb{H}}$. This last expression is bounded courtesy of Lemma 7.3 in [14] by $c_{0}\left(r^{-1 / 2+4 \sigma}+|\lambda|\right)^{2}\left(\rho^{v / 4}+r^{-8}\right)$. In particular, if $\rho>r^{-2}$, then what was just written is bounded by what is written on the right hand side of (3-8) if $\delta \leq v / 4$.

Part 4 Write $\mathfrak{f} \in \mathbb{H}$ again as $\mathfrak{f}^{\perp}+t_{\xi}(\theta)$ with $\mathfrak{f}^{\perp} \in \mathbb{H}_{\xi} \frac{\perp}{\xi}$. Use $\Pi_{\xi}$ to denote the $L^{2}-$ orthogonal projection to the image of $t_{\xi}$, either in $\mathbb{L}$ or in $\mathbb{H}$. As done in Part 7 of Section 6.a in [14], introduce $\mathbb{L}_{\xi}^{\frac{1}{\xi}}$ to denote $\left(1-\Pi_{\xi}\right) \mathbb{L}$, this the $L^{2}$ orthogonal complement of the kernel of the adjoint, $t_{\xi}^{\dagger}: \mathbb{L} \rightarrow \mathcal{L}^{2}$. When considering the operator $\mathfrak{D}_{\xi}$, it proves useful to view it with the help of its projections in $\mathbb{L}_{\xi}^{+}$and via $t_{\xi}^{\dagger}$ in $\mathcal{L}^{2}$. To this end write

$$
\begin{array}{ll}
\text { - } & \left(1-\Pi_{\xi}\right) \mathfrak{D}_{\xi} \mathfrak{f}=\mathfrak{D}_{\xi}^{\perp} \mathfrak{f}^{\perp}+p(\theta) . \\
\text { - } & t_{\xi}^{\dagger}\left(\mathfrak{D}_{\xi} \mathfrak{f}\right)=p^{\prime}\left(\mathfrak{f}^{\perp}\right)+\Delta \theta .
\end{array}
$$

To start the analysis of the terms on the right hand side of (3-11), use the small size of $\mathfrak{h}(\xi)$ with Lemma 6.1 in [14] to see that

$$
\left\|\mathfrak{D}_{\xi}^{\perp} \mathfrak{f}^{\perp}\right\|_{2} \geq c_{0}\left\|\mathfrak{f}^{\perp}\right\|_{\mathbb{H}} .
$$


This same lemma from [14] also implies that $\mathfrak{D}_{\xi}^{\perp}$ is invertible as a map from $\mathbb{H} \frac{\perp}{\xi}$ to $\mathbb{L}_{\xi} \frac{\perp}{\xi}$. Meanwhile, (6-6)-(6-7) from [14], the descriptions in the latter's Section 6.d of $\mathfrak{h}$, and what is said in Lemma 3.10 of [14] imply that

$$
\|p(\theta)\|_{2} \leq c_{0} \rho_{*}\left(\|\nabla \theta\|_{\mathcal{L}^{2}}+r^{1 / 2}\|\theta\|_{\mathcal{L}^{2}}\right) \leq c_{0} r^{3 \sigma}\|\theta\|_{\mathcal{K}_{1}^{2}}
$$

These same parts of [14] also find that

$$
\left\|p^{\prime}\left(\mathfrak{f}^{\perp}\right)\right\|_{\mathcal{L}^{2}} \leq c_{0} \rho_{*}\left\|\mathfrak{f}^{\perp}\right\|_{\mathbb{H}} \leq c_{0} r^{-1 / 2+3 \sigma}\left\|\mathfrak{f}^{\perp}\right\|_{\mathbb{H}} .
$$

Note that the factor $r^{3 \sigma}$ is absent in both (3-13) and (3-14) when each $C \in \mathcal{C}$ component of $\theta$ has support on $C-\left(\bigcup_{\mathcal{E} \in \mathcal{E}_{C}} \mathcal{E}_{2 R}\right)$.

The operator $\Delta$ is described in part by the next lemma. The latter refers to the inner product $\mathcal{K}^{2}$ on $\mathcal{K}_{1}^{2}$ that is obtained by replacing in (5-13) of [14] each occurrence of $\|\cdot\|_{\mathcal{K}}$ with the corresponding $L^{2}$ norm.

Lemma 3.2 There exists $\kappa>1$ such that if the constants $R$ and $r$ used to construct $\mathfrak{d}_{\Sigma}$ obey $R \geq \kappa, r \geq \kappa^{2}$, and $|\lambda| \leq \kappa^{-1}$ then the operator $\Delta$ is Fredholm as a map from $\mathcal{K}_{1}^{2}$ to $\mathcal{L}^{2}$ with trivial cokernel and kernel dimension $I\left(\Theta_{-}, \Theta_{+} ; Z\right)$. Moreover, $\|\Delta \theta\|_{\mathcal{L}^{2}} \geq \kappa^{-1}\|\theta\|_{\mathcal{K}_{1}^{2}}$ when $\theta$ is $\mathcal{K}^{2}$-orthogonal to the kernel of $\Delta$.

This lemma is proved in Part 6 below.

What with (3-5), (3-9), (3-12)-(3-14) and Lemma 3.2, all of the relevant questions about $\mathfrak{D}_{\Sigma}$ can be rephrased as questions about $\Delta$. The following lemma states something more precise.

Lemma 3.3 There exists $\kappa>1$ such that if the constants $R$ and $r$ used to construct $\mathfrak{d}_{\Sigma}$ obey $R \geq \kappa, r \geq \kappa^{2}$, and $|\lambda| \leq \kappa^{-1}$, then $\mathfrak{D}_{\Sigma}$ has trivial cokernel and the composition of $t$ with $L^{2}$-orthogonal projection maps the kernel of $\Delta$ isomorphically onto the kernel of $\mathfrak{D}_{\Sigma}$. As a consequence, the index of $\mathfrak{D}_{\Sigma}$ is equal to that of $\Delta$; thus $I\left(\Theta_{-}, \Theta_{+} ; Z\right)$.

The assertions made by Proposition 3.1 are direct corollaries to this last lemma.

Proof of Lemma 3.3 Use (3-5) and (3-12) to conclude that the linear map $\mathfrak{f}^{\perp} \rightarrow$ $\mathfrak{D}_{\Sigma}^{\perp} \mathfrak{f}^{\perp}=\mathfrak{D}_{\xi}^{\perp} \mathfrak{f}^{\perp}+r^{1 / 2}\left(1-\Pi_{\xi}\right)\left(\mathfrak{e}_{\mathfrak{q}}^{*} \mathfrak{f}^{\perp}\right)$ is invertible, with $\left\|\left(\mathfrak{D}_{\Sigma}^{\perp}\right)^{-1}(\cdot)\right\|_{\mathbb{H}} \leq c_{0}\|\cdot\|_{2}$. This understood, it follows that if $\mathfrak{y} \in \mathbb{L}$ and $\mathfrak{f}=\mathfrak{f}^{\perp}+t(\theta) \in \mathbb{H}$ obeys $\mathfrak{D}_{\Sigma} \mathfrak{f}=\mathfrak{y}$, then

$$
f^{\perp}=\left(\mathfrak{D}_{\Sigma}^{\perp}\right)^{-1}\left(1-\Pi_{\xi}\right)\left(\mathfrak{y}-r^{1 / 2} \mathfrak{e}_{\mathfrak{q}}^{*} t(\theta)-p(\theta)\right) .
$$

Meanwhile, $\theta$ obeys the equation

$$
\Delta(\theta)+e(\theta)=t^{\dagger}(\mathfrak{y})-p^{\prime}\left(\left(\mathfrak{D} \frac{\perp}{\Sigma}\right)^{-1}\left(1-\Pi_{\xi}\right) \mathfrak{y}\right),
$$


where

$$
e(\theta)=p^{\prime}\left(\left(\mathfrak{D}_{\Sigma}^{\frac{1}{\Sigma}}\right)^{-1}\left(r^{1 / 2}\left(1-\Pi_{\xi}\right)\left(\mathfrak{e}_{\mathfrak{q}}^{*} t(\theta)\right)+p(\theta)\right)\right)+r^{1 / 2} t^{\dagger}\left(\mathfrak{e}_{\mathfrak{q}}^{*} t(\theta)\right) .
$$

Note in particular that the operator $e$ obeys

$$
\|e(\theta)\|_{\mathcal{L}^{2}} \leq c_{0}\left(r^{-1 / 2+8 \sigma}+|\lambda|\right)\|\theta\|_{\mathcal{K}_{1}^{2}} .
$$

Indeed, this follows by first using (3-9) to bound $r^{1 / 2} t^{\dagger}\left(\mathfrak{e}_{\mathfrak{q}}^{*} t(\theta)\right)$ and then the following two additional facts: First, the operator

$$
\theta \rightarrow p^{\prime}\left(\left(\mathfrak{D} \frac{\perp}{\Sigma}\right)^{-1} r^{1 / 2}\left(1-\Pi_{\xi}\right)\left(\mathfrak{e}_{\mathfrak{q}}^{*} t(\theta)\right)\right)
$$

has norm bounded by $c_{0}\left(r^{-1 / 2+8 \sigma}+|\lambda|\right)^{2}$, this because of (3-9), (3-14) and the bound on the norm of $\left(\mathfrak{D} \frac{\perp}{\Sigma}\right)^{-1}$. Second, the operator

$$
\theta \rightarrow p^{\prime}\left(\left(\mathfrak{D} \frac{\perp}{\Sigma}\right)^{-1} p(\theta)\right)
$$

has norm bounded by $c_{0} r^{-1 / 2+3 \sigma} r^{3 \sigma}$, this because of (3-13), (3-14) and the bound on the norm of $\left(\mathfrak{D} \frac{\perp}{\Sigma}\right)^{-1}$.

Given what is asserted by Lemma 3.2, the claims made by Lemma 3.3 follow directly from (3-16) and (3-17).

Part 5 This part describes the operator $\Delta$ in preparation for the upcoming proof of Lemma 3.2 To begin, write $\theta \in \mathcal{K}_{1}^{2}$ as $\theta=\left(\left(\theta_{C}\right)_{C \in \mathcal{C}},\left(\theta_{\gamma-}\right)_{\gamma \in \Xi_{\Sigma-}},\left(\theta_{\gamma+}\right)_{\gamma \in \Xi_{\Sigma_{+}}}\right)$and write the component of $\Delta(\theta)$ as $\Delta(\theta)=\left(\left(\Delta_{C}\right)_{C \in \mathcal{C}},\left(\Delta_{\gamma-}\right)_{\gamma \in \Xi_{\Sigma-}},\left(\Delta_{\gamma+}\right)_{\gamma \in \Xi_{\Sigma+}}\right)$. The three steps that follow supply a description of these components.

Step 1 Suppose that $C \in \mathcal{C}$ is not $\mathbb{R}$-invariant. View $\theta_{C}$ as a section of $C$ 's normal bundle $N$, and view $\Delta_{C}$ as a section of $N \otimes T^{0,1} C$. A virtual replay of what is said in Parts 4 and 5 of Section 2.c that lead to (2-80) finds that $\Delta_{C}$ appears as in (2-80):

$$
\Delta_{C}(\theta)=\bar{\partial} \theta_{C}+v_{C} \theta_{C}+\mu_{C} \bar{\theta}_{C}+\mathfrak{e},
$$

where the $L_{1}^{2}$ norm of $\mathfrak{e}$ on $C-\left(\bigcup_{\mathcal{E} \in \mathcal{E}_{C}} \mathcal{E}_{2 R}\right)$ is bounded by $c_{0}\left(r^{-\sigma / 4}+|\lambda|\right)\|\theta\|_{\mathcal{K}_{1}^{2}}$.

Step 2 Suppose next that $C \in \mathcal{C}$ is an $\mathbb{R}$-invariant cylinder, thus $\mathbb{R} \times \gamma$ for some Reeb orbit $\gamma$. Let $m$ denote the associated positive integer. Use the coordinates in (1-5) of [14] to identify the tangent space in $\mathfrak{C}_{m}$ at the symmetric vortex with $\mathbb{C}^{m}$. Use $C$ 's identification with $\mathbb{R} \times \gamma=\mathbb{R} \times S^{1}$ and $C$ 's normal bundle identification as $C \times \mathbb{C}$, these as described in Section 4.a of [14], to identify $\theta_{C}$ and $\Delta_{C}$ as maps from the $|w| \leq 4 R$ part if $\mathbb{R} \times S^{1}$ to $\mathbb{C}^{m}$. In the case $m=1$, the arguments that lead to (2-80) can be used almost to verbatim prove that $\Delta_{C}$ has the form given in (3-20) with 
the pair $\left(v_{C}, \mu_{C}\right)$ equal to $\gamma$ 's pair $(\nu, \mu)$. In the case when $m>1$, the arguments that lead to (2-80) together with those that give the formula for $\mathrm{D} \theta$ in Lemma 2.1 prove that $\Delta_{C}$ has the following form: Let $\Delta_{C q}$ and $\theta_{C q}$ denote the $q$-th coordinates components in $\mathbb{C}^{m}$ of the maps $\theta_{C}$ and $\Delta_{C}$. Then

$$
\Delta_{C q}=\bar{\partial} \theta_{C q}+\frac{q}{2} \mathrm{R} \theta_{C q}+\mathfrak{e},
$$

where $\mathrm{R}$ here denotes the rotation number of $\gamma$. Meanwhile, $\mathfrak{e}$ is such that its $L_{1}^{2}$ norm is bounded by $c_{0}\left(r^{-\sigma / 4}+|\lambda|\right)\|\theta\|_{\mathcal{K}_{1}^{2}}$.

Step 3 Consider next $\Delta_{\gamma-}$ for a given $\gamma \in \Xi_{\Sigma-}$. A very similar description holds for $\Delta_{\gamma+}$ when $\gamma \in \Xi_{\Sigma+}$. The $\left\{\Delta_{\gamma+}\right\}_{\gamma \in \Xi_{\Sigma+}}$ descriptions are left to the reader.

Suppose first that the associated integer $m_{\gamma-}$ is equal to 1 . To describe $\Delta_{\gamma-}$, it proves convenient to view both $\Delta_{\gamma-}(\theta)$ and $\theta_{\gamma-}$ as maps to $\mathbb{C}$ from $(-\infty,-R) \times S^{1}$ to $\mathbb{C}$. This is done using the coordinate $\sigma_{1}$ in (1-5) of [14]. Given the fact that $\|\xi\|_{\mathcal{K}} \leq c_{0} r^{-1 / 2+8 \sigma}$, a very slight modification of the arguments leading to (2-81) finds that $\Delta_{\gamma-}(\theta)$ is given by

$$
\Delta_{\gamma-}(\theta)=\bar{\partial} \theta_{\gamma-}+v \theta_{\gamma-}+\mu \bar{\theta}_{\gamma-}+\mathfrak{e},
$$

where the $L^{2}$ norm of $\mathfrak{e}$ on the $s \leq-R$ part of $U_{\gamma-}$ is bounded by $c_{0}\left(r^{-\sigma / 4}+|\lambda|\right)\|\theta\|_{\mathcal{K}_{1}^{2}}$. Suppose next that $m_{\gamma-}>1$. The simplest case to analyze here is that when $\mathcal{C}$ contains the cylinder $\mathbb{R} \times \gamma$ and its associated integer is $m_{\gamma-}$. The arguments for the $\mathrm{D} \theta$ formula in Lemma 2.1 can be re-employed to prove that $\Delta_{\gamma-}(\theta)$ in this case has the form given in (3-21) on the $q$-th summand in $\mathbb{C}^{m_{\gamma}}$.

Assume again that $m_{\gamma-}>1$ and suppose $\mathcal{E}_{\gamma-}$ contains an end that is not part of $\mathbb{R} \times \gamma$. Reintroduce the notation from Section 5.c of [14] to view $c_{\gamma-}{ }^{*} T_{1,0} \mathfrak{C}_{m_{\gamma-}}$ over the cylin$\operatorname{der} I_{k} \times S^{1}$ for any given $k \in\left\{1, \ldots, N_{\gamma-}\right\}$ as $\mathfrak{c}_{k}^{*} T_{1,0} \mathfrak{C}_{m_{k}} \oplus\left(\bigoplus_{\mathcal{E} \in \mathcal{E}_{\gamma-}-\mathcal{E}_{k}} \pi_{*}\left(\left.N\right|_{E}\right)\right)$. Use this isomorphism to write $\theta_{\gamma-}$ as $\left(\theta_{k}, \pi_{*}\left(\theta_{\Sigma k}\right)\right)$ and $\Delta_{\gamma-}$ as $\left(\Delta_{k}, \pi_{*}\left(\Delta_{\Sigma k}\right)\right)$. Then

$$
\Delta_{k}=\bar{\partial}_{\mathfrak{c}_{k}} \theta_{k}+\left.\left(\nabla_{\theta_{R}} \nabla^{1,0} h\right)\right|_{c_{k}}+\mathfrak{e}
$$

where $\epsilon_{t}$ is the $v=\frac{1}{2} \mathrm{R}$ version of the function $\hbar_{v}$ on $\mathfrak{C}_{m_{k}}$ given in (2-41), and where $\mathfrak{e}$ is such that its $L^{2}$ norm on $I_{k} \times S^{1}$ is bounded by $c_{0}\left(r^{-\sigma / 4}+|\lambda|\right)\|\theta\|_{\mathcal{K}_{1}^{2}}$. Meanwhile, if $\mathcal{E} \in \mathcal{E}_{\gamma-}-\mathcal{E}_{k}$, then $\mathcal{E}$ 's component of $\Delta_{\Sigma k}$ is given by (3-21). These assertions can be proved using, once again, arguments that differ little from those used to derive the formula for $\mathrm{D} \theta$ in Lemma 2.1. The details of all of this offer nothing new and are omitted. Reintroduce the map $\mathfrak{c}_{k 0}: \mathbb{R} \times S^{1} \rightarrow \mathfrak{C}_{m_{k}}$ from Section 7.f of [14]. As is noted there, it follows from Constraint 2 in Section 4.b of [14] that the distance in $\mathfrak{C}_{m_{k}}$ 
between $\left.\mathfrak{c}_{k}\right|_{(w, t)}$ and $\left.\mathfrak{c}_{k 0}\right|_{(w, t)}$ is bounded by $c_{0} r^{-\sigma / 4}$ at each $(w, t) \in I_{k} \times S^{1}$. As a consequence, the map $\exp _{\mathfrak{c}_{k 0}}$ from Part 8 in Section 2.a of [14] can be used to identify the $\mathfrak{c}_{k 0}$ and $\mathfrak{c}_{k}$ pullbacks over $I_{k} \times S^{1}$ of $T_{1,0} \mathfrak{C}_{m_{k}}$ when $r \geq c_{0}$. With this identification understood, then $\Delta_{k}$ in (3-23) can just as well be written as

$$
\Delta_{k}=\bar{\partial}_{\mathfrak{c}_{k 0}} \theta_{k}+\left.\left(\nabla_{\theta_{k \mathbb{R}}} \nabla^{1,0} h\right)\right|_{\mathfrak{c}_{k 0}}+\mathfrak{e}
$$

where $\mathfrak{e}$ here differs from its namesake in (3-23) but obeys $\|\mathfrak{e}\|_{\mathcal{L}^{2}} \leq c_{0}\left(r^{-\sigma / 4}+|\lambda|\right)\|\theta\|_{\mathcal{K}_{1}^{2}}$.

Part 6 This part of the subsection consists of the.

Proof of Lemma 3.2 If $\gamma \in \Xi_{\Sigma-}$ and $m_{\gamma-}=1$, set $V_{\gamma-, 1}=0$. Likewise, set $V_{\gamma+}=0$ if $\gamma \in \Xi_{\Sigma+}$ and $m_{\gamma+}=1$. Now suppose that $\gamma \in \Xi_{\Sigma-}$ and $m_{\gamma-}>1$. If there is just one end of $\Sigma$ in $U_{\gamma-}$ and the latter is an end of $\mathbb{R} \times \gamma$, then set $V_{\gamma-, 1}=0$. If such is not the case, introduce the notation from Section 5.c in [14]. If $k \in\left\{1, \ldots, N_{\gamma-}\right\}$, use $V_{\gamma-, k}$ to denote the kernel of the operator

$$
\xi \rightarrow \bar{\partial}_{\mathfrak{c}_{k 0}} \xi+\left.\left(\nabla_{\xi_{\mathbb{R}}} \nabla^{1,0} h\right)\right|_{\mathfrak{c}_{k 0}}
$$

when viewed as mapping $L_{1}^{2}\left(\mathbb{R} \times S^{1} ; \mathfrak{c}_{k 0}^{*} T_{1,0} \mathfrak{C}_{m_{k}}\right)$ to $L^{2}\left(\mathbb{R} \times S^{1} ; \mathfrak{c}_{k 0}^{*} T_{1,0} \mathfrak{C}_{m_{k}}\right)$. Note that Lemma 7.5 in [14] asserts that this operator is Fredholm with trivial cokernel. In the case when $\gamma \in \Xi_{\Sigma+}$, define the analogous vector spaces $V_{\gamma+, k}$.

Define $V_{0}$ as in (7-43) of [14]. Note in this regard that the kernel of $\mathcal{D}_{C}$ is trivial when $C$ is an $\mathbb{R}$-invariant cylinder. Mimic what is done in Section 7.f of [14] to define the maps $q: \mathcal{K}_{1}^{2} \rightarrow V_{0}$ and $\phi_{0}: V_{0} \rightarrow \mathcal{K}_{1}^{2}$. With only cosmetic changes, the arguments from Section 7.f of [14] establish the following when $r \geq c_{0}$ and $R \geq c_{0}$ : First, $q$ maps onto $V_{0}$ and, in addition,

$$
\left\|q\left(\phi_{0}(\xi)\right)-\xi\right\|_{L^{2}} \leq c_{0} r^{-1 / c_{0}}\|\xi\|_{L^{2}} \text { for all } \xi \in V_{0}
$$

Meanwhile, it follows from what is said about $\Delta$ in Part 5 above that

$$
\left\|\Delta \phi_{0}(\xi)\right\|_{\mathcal{L}^{2}} \leq c_{0}\left(r^{-1 / c_{0}}+|\lambda|\right)\|\xi\|_{L^{2}} \text { for all } \xi \in V_{0} .
$$

Finally, the arguments given in Sections $7 \mathrm{~g}$ and $7 \mathrm{~h}$ in [14] can be applied with only notational changes to prove the following: If $r \geq c_{0}, R \geq c_{0}$ and $|\lambda| \leq c_{0}{ }^{-1}$, then

- $\Delta$ maps the kernel of $q$ onto $\mathcal{L}^{2}$.

- $\|\Delta(\theta)\|_{\mathcal{L}^{2}} \geq c_{0}{ }^{-1}\|\theta\|_{\mathcal{K}_{1}^{2}}$ if $q(\theta)=0$.

These last points imply that the kernel of $\Delta$ is isomorphic to $V_{0}$, that $\Delta$ has trivial cokernel, and that $\|\Delta(\theta)\|_{\mathcal{L}^{2}} \geq c_{0}{ }^{-1}\|\theta\|_{\mathcal{K}_{1}^{2}}$ if $\theta$ is $\mathcal{L}^{2}$ orthogonal to the kernel of $\Delta$. In this regard, the isomorphism between $V_{0}$ and the kernel of $\Delta$ can be taken so 
as to send any given $\xi \in V_{0}$ to $\phi_{0}(\xi)+\iota(\xi)$ where $\iota(\xi) \in \operatorname{kernel}(q)$ obeys $\|\iota\|_{\mathcal{K}_{1}^{2}} \leq$ $c_{0}\left(r^{-1 / c_{0}}+|\lambda|\right)\|\xi\|_{L^{2}}$.

Granted what just been said, all that remains is to verify that $\operatorname{dim}\left(V_{0}\right)$ is equal to $I\left(\Theta_{-}, \Theta_{+} ; Z\right)$. This task is done in the six steps that follow.

Step 1 Suppose that $C \in \mathcal{C}$ is not $\mathbb{R}$-invariant. Introduce $\mathcal{D}_{C}$ as depicted in (1-10), viewed as a Fredholm map from $L_{1}^{2}(C ; N)$ to $L^{2}\left(C ; N \otimes T^{0,1} C\right)$. As $\mathcal{D}_{C}$ has trivial cokernel, the dimension of its kernel is the index of $\mathcal{D}_{C}$. This index can be computed using the formula from Theorem 5.1 in [6]. What with Lemma 2.3, the result can be put in the following schematic form:

$$
\begin{aligned}
\operatorname{index}\left(\mathcal{D}_{C}\right)=- & \sum_{\gamma \in \Xi_{\Sigma-}} \sum_{\mathcal{E} \in \mathcal{E}_{C} \cap \mathcal{E}_{\gamma-}} z_{\mathcal{E}} \\
& +\sum_{\gamma \in \Xi_{\Sigma+}} \Sigma_{\mathcal{E} \in \mathcal{E}_{C} \cap \mathcal{E}_{\gamma+}} z_{\mathcal{E}}-\left\langle c_{1}, C\right\rangle+\operatorname{deg}\left(N_{C}\right),
\end{aligned}
$$

where the notation is as follows: First, $\left\langle c_{1}, C\right\rangle$ denotes the Euler class of a section of the bundle $\left.K\right|_{C}$ with it understood that the section is given on $C$ 's intersection with any given $U_{\gamma \pm}$ as the restriction of the projection to $K$ of the vector field $\frac{\partial}{\partial z}$ Second, $\operatorname{deg}\left(N_{C}\right)$ denotes the Euler class of $C$ 's normal bundle as defined using a section whose restriction to $C$ 's intersection with any given $U_{\gamma \pm}$ is the projection to $N$ of this same $\partial / \partial z$ Finally, what is written as $z_{\mathcal{E}}$ is defined as follows: If the associated Reeb orbit $\gamma$ is hyperbolic, then $z_{\mathcal{E}}$ is the rotation number of $\gamma$. If the Reeb orbit $\gamma$ is elliptic, then $z_{\mathcal{E}}=2 k_{q_{\mathcal{E}}}^{<}+1$ where $q_{\mathcal{E}}$ denotes the integer that is described in Constraint 3 of Section 4.b in [14], where $\mathrm{R}$ is $\gamma$ 's rotation number, and where $k_{q \mathrm{R}}^{<}$ is the greatest integer that is less than $q$ R.

Step 2 Consider $\operatorname{dim}\left(V_{\gamma-, k}\right)$ in the case when $\gamma \in \Xi_{\Sigma-}$ and $k \in\left\{1, \ldots, N_{\gamma-}\right\}$. Assume that $\mathcal{E}_{\gamma-}$ contains at least one end that is not part of $\mathbb{R} \times \gamma$. If such is not the case, then there is only $V_{\gamma-, 1}$ and the latter has dimension zero. Reintroduce the notation from the proof of Lemma 7.5 in Section 7.i of [14]. This step considers the case that is discussed in Step 2 of the proof of Lemma 7.5 in [14]. Thus, $\mathcal{E}_{\gamma-\text { consists of a }}$ single end, and the latter, $\mathcal{E}$, has $q_{\mathcal{E}}=m_{\gamma-}$. In this case, $N_{\gamma-}=1$ and the space $V_{\gamma, 1}$ can be written as a direct sum $\bigoplus_{1 \leq q \leq q_{\mathcal{E}}} V_{q}$ where $V_{q}$ denotes the $L^{2}$ kernel of the operator depicted in (7-60) of [14]. It follows from (7-61) of [14] that any function annihilated by the operator in (7-60) of [14] is a linear combination of those that have the form $(w, t) \rightarrow e^{u(w)+k(w+i t)}$ where $k \in \mathbb{Z}$ and where the function $u(\cdot)$ obeys

$$
\begin{aligned}
& \text { - } u(w) \rightarrow-q \mathrm{R} w+o(1) \text { as } w \rightarrow-\infty, \\
& \text { - } u(w) \rightarrow-\left(\mathrm{R}+(q-1) k_{\mathcal{E}} / q_{\mathcal{E}}\right) w+o(1) \text { as } w \rightarrow+\infty .
\end{aligned}
$$


Here, $k_{\mathcal{E}}$ is the least integer that is greater than $q_{\mathcal{E}} \mathrm{R}$. It follows from (3-30) that the function $e^{u(w)+k(w+i t)}$ is square integrable if and only if

$$
q \mathrm{R}<k<q \mathrm{R}+(q-1)\left(k_{\mathcal{E}} / q_{\mathcal{E}}-\mathrm{R}\right)=q k_{\mathcal{E}} / q_{\mathcal{E}}-\left(k_{\mathcal{E}} / q_{\mathcal{E}}-\mathrm{R}\right) .
$$

As is argued next, this inequality is satisfied by at most one integer, $k_{q_{\mathrm{R}}}^{>}$, and such is the case if and only if $k_{q \mathrm{R}}^{>} / q<k_{\mathcal{E}} / q_{\mathcal{E}}$.

To prove that the only possible integer that obeys both sides of (3-31) is $k_{q \mathrm{R}}^{>}$, note first that the left hand inequality requires $k \geq k_{q \mathrm{R}}^{>}$. On the other hand, the ratio $k_{\mathcal{E}} / q_{\mathcal{E}}$ is less than $\mathrm{R}+1 / q_{\mathcal{E}}$ which is less than $k_{q \mathrm{R}}^{>} / q+1 / q_{\mathcal{E}}$; thus the right hand side of (3-31) is strictly less than $k_{q \mathrm{R}}^{>}+1$. As a consequence, there is at most one integer, namely $k_{q \mathrm{R}}^{>}$, that obeys both sides of (3-31).

To see only one such integer if and only if $k_{q \mathrm{R}}^{>} / q<k_{\mathcal{E}} / q_{\mathcal{E}}$, note first that the necessity of this condition follows directly from the fact that the far right term in (3-31) is negative. To see that (3-31) holds when $k_{q \mathrm{R}}^{>} / q<k_{\mathcal{E}} / q_{\mathcal{E}}$, rewrite this inequality as $q_{\mathcal{E}} k_{q \mathrm{R}}^{>} / q<k_{\mathcal{E}}$. Thus, $k_{\mathcal{E}}=q_{\mathcal{E}} k_{q \mathrm{R}}^{>} / q+p / q$ for some integer $p>0$ and so $k_{q \mathrm{R}}^{>}=q k_{\mathcal{E}} / q_{\mathcal{E}}-p / q_{\mathcal{E}}$. Meanwhile, $k_{\mathcal{E}} / q_{\mathcal{E}}-R<1 / q_{\mathcal{E}}$, and so the far right hand term in (3-31) is strictly greater than $-1 / q_{\mathcal{E}}$.

Let $n_{q}=1$ if $k_{q \mathrm{R}}^{>} / q<k_{\mathcal{E}} / q_{\mathcal{E}}$ and zero otherwise. It follows from what was just said that

$$
\operatorname{dim}\left(V_{\gamma-, 1}\right)=2 \sum_{1 \leq q \leq q_{\mathcal{E}}} n_{q}
$$

To put this sum in perspective, let $k_{q *}$ denote the least integer greater than $q k_{\mathcal{E}} / q_{\mathcal{E}}$. Then $k_{q \mathrm{R}}^{>}-k_{q *}-n_{q}$. This understood,

$$
\operatorname{dim}\left(V_{\gamma-, 1}\right)=-\sum_{1 \leq q \leq q_{\mathcal{E}}} 2 k_{q \mathrm{R}}^{>}+2 \sum_{1 \leq q \leq q_{\mathcal{E}}} k_{q *} .
$$

As explained to the author by Noam Elkies, the right most sum in (3-33) is

$$
2 \sum_{1 \leq q<q_{\mathcal{E}}} k_{q^{*}}=\left(q_{\mathcal{E}}-1\right)\left(k_{\mathcal{E}}+1\right),
$$

this a consequence of the fact that $q k_{\mathcal{E}} / q_{\mathcal{E}}+\left(q_{\mathcal{E}}-q\right) k_{\mathcal{E}} / q_{\mathcal{E}}=k_{\mathcal{E}}$. Granted (3-34), reintroduce $z_{\gamma, q}=2 k_{q \mathrm{R}}^{<}+1=2 k_{q \mathrm{R}}^{>}-1$ and so write

$$
\operatorname{dim}\left(V_{\gamma, 1}\right)=-\sum_{1 \leq q \leq m_{\gamma-}} z_{\gamma, q}+z_{\mathcal{E}}+k_{\mathcal{E}}\left(q_{\mathcal{E}}-1\right)
$$


Note for a subsequent step that the far right term in (3-35) has the following geometric interpretation: Let $S \subset \mathbb{C}^{*} \times \mathbb{C}$ denote the nonsingular curve given in terms of the coordinates $\left(x=e^{(w+i t)}, Z\right)$ as

$$
x^{k_{\mathscr{E}}}-z^{q_{\mathscr{E}}}=1 .
$$

Let $\mathfrak{s}$ denote the projection of the vector field $\partial / \partial z$ onto the normal bundle of $S$. This vector field is nonvanishing where $|x| \ll 1$ and where $|x| \gg 1$. Thus, it has a well defined Euler class. The latter is equal to $k_{\mathcal{E}}\left(q_{\mathcal{E}}-1\right)$.

Step 3 This step considers $\operatorname{dim}\left(V_{\gamma-, k}\right)$ for the case that is discussed in Step 3 of the proof of Lemma 7.5 in [14]. In this case, there are $n>1$ ends in $\mathcal{E}_{\gamma-}$, each with the same integer $q_{\mathcal{E}}$. Again there is just $V_{\gamma-, 1}$. Given what is said there, a virtual repeat of the arguments just given find that

$$
\operatorname{dim}\left(V_{\gamma-, 1}\right)=2 \sum_{1 \leq q<n q_{\mathcal{E}}} n_{q},
$$

where $n_{q}$ is the number of integers that obey (3-31). To say something more about $n_{q}$, remark that (3-31) is obeyed if and only if $k$ satisfies

$$
k_{q \mathrm{R}}^{>} \leq k<q k_{\mathcal{E}} / q_{\mathcal{E}} .
$$

Indeed, necessity of (3-38) for the left hand inequality in (3-31) follows because there are no integers between $q \mathrm{R}$ and $k_{q \mathrm{R}}^{>}$. The necessity of (3-38) for the right hand inequality in (3-31) follows since $-\left(k_{\mathfrak{E}} / q_{\mathcal{E}}-\mathrm{R}\right)<0$. The sufficiency of (3-38) for the left hand inequality in (3-31) follows since $q \mathrm{R}<k_{q \mathrm{R}}^{>}$. The sufficiency of (3-38) for the right hand inequality is argued in Step 2.

It follows from (3-38) that

$$
n_{q}=k_{q^{*}}-k_{q \mathrm{R}}^{>}
$$

where $k_{q^{*}}$ denotes the least integer greater than or equal to $q k_{\mathcal{E}} / q_{\mathcal{E}}$. This understood, (3-36) can be rewritten as

$$
\operatorname{dim}\left(V_{\gamma-, 1}\right)=-\sum_{1 \leq q \leq m_{\gamma-}} z_{\gamma, q}-n q_{\mathcal{E}}+2 \sum_{1 \leq q \leq n q_{\mathcal{E}}} k_{q^{*}}
$$

The sum on the far right can be evaluated as was (3-34) to find that

$$
\operatorname{dim}\left(V_{\gamma, 1}\right)=-\Sigma_{1 \leq q \leq m_{\gamma-}} z_{\gamma, q}+n\left(2 k_{\mathcal{E}}-1\right)+n k_{\mathcal{E}}\left(n q_{\mathcal{E}}-1\right) .
$$

Since $\left(2 k_{\mathcal{E}}-1\right)$ is equal to $z_{\mathcal{E}}$, what is written in (3-41) is

$$
\operatorname{dim}\left(V_{\gamma, 1}\right)=-\sum_{1 \leq q \leq m_{\gamma-}} z_{\gamma, q}+\sum_{\mathcal{E} \in \mathcal{E}_{\gamma-}} z_{\mathcal{E}}+n k_{\mathcal{E}}\left(n q_{\mathcal{E}}-1\right) .
$$


For future reference, note that the right most term in (3-42) also has a geometric interpretation as the Euler class of a section of the normal bundle of a curve in $\mathbb{C}^{*} \times \mathbb{C}$. In this case, the curve, $S$, is the locus of pairs $(x, z)$ where

$$
x^{n k_{\mathcal{E}}}-z^{n q_{\mathcal{E}}}=1 \text {. }
$$

Consider again the projection, $\mathfrak{s}$, of $\partial / \partial z$ to the normal bundle of $S$. The latter vanishes at each of the $n k_{\mathcal{E}}$ points of $S$ where $z=0$ with degree $n q_{\mathcal{E}}-1$. This understood, its Euler class is $n k_{\mathcal{E}}\left(n q_{\mathcal{E}}-1\right)$.

Step 4 This step corresponds to the case that is considered in Step 4 of the proof of Lemma 7.5 in [14]. Consider $\operatorname{dim}\left(V_{\gamma-, k}\right)$. Given (7-71) in [14] and what is said in the final paragraph of Step 4 of the proof of Lemma 7.5 in [14], the arguments from the previous two steps can be employed with only notational changes to prove that

$$
\operatorname{dim}\left(V_{\gamma-, k}\right)=2 \Sigma_{m_{k-1}<q \leq m_{k-1}+n q_{\mathcal{E}}}\left(k_{q^{*}}-k_{q \mathrm{R}}^{>}\right),
$$

where $k_{q^{*}}$ denotes the least integer that is greater than or equal to $q k_{\mathcal{E}} / q_{\mathcal{E}}$. A variation of the argument used for (3-34) finds the $k_{q *}$ sum in (3-44) equal to

$$
2 n m_{k-1} k_{\mathcal{E}}+n k_{\mathcal{E}}\left(n q_{\mathcal{E}}-1\right)+n\left(2 k_{\mathcal{E}}-1\right)+n q_{\mathcal{E}} .
$$

As a consequence, what is written in (3-44) can be rewritten as

$$
\operatorname{dim}\left(V_{\gamma-, k}\right)=-\sum_{m_{k-1}<q \leq m_{k}} z_{\gamma, q}+\sum_{\mathcal{E} \in \mathcal{E}_{k}} z_{\mathcal{E}}+n k_{\mathcal{E}}\left(n q_{\mathcal{E}}-1+2 m_{k-1}\right) .
$$

The right most term in (3-46) has the following geometric interpretation: Let $S_{k}=$ $S \cup S^{\prime} \subset \mathbb{C}^{*} \times \mathbb{C}$ denote the reducible curve given as follows: First, $S$ is given in terms of the coordinates $(x, z)$ by (3-43). Second, $S^{\prime}$ is the disjoint union of $m_{k-1}$ disjoint, constant $z$ cylinders, each with $z>0$ and $|z| \ll 1$. The right most term in (3-46) is the sum of two terms: The first, $n k_{\mathcal{E}}\left(n q_{\mathcal{E}}-1\right)$ is again the Euler class of the normal bundle of $S$ as defined by the restriction of $\partial / \partial z$ The second, $2 n k_{\mathcal{E}} m_{k-1}$, is twice the intersection number between $S$ with $S^{\prime}$.

Step 5 It follows from what has been said in Steps 2-4 that

$$
\sum_{1 \leq k \leq N_{\gamma-}} \operatorname{dim}\left(V_{\gamma-, k}\right)=-\sum_{1 \leq q \leq m_{\gamma-}} z_{\gamma, q}+\sum_{\mathcal{E} \in \mathcal{E}_{\gamma-}} z_{\mathcal{E}}+\operatorname{deg}\left(N_{Z \cap U_{\gamma-}}\right),
$$

where the far right term is short hand for the degree of the normal bundle of a certain surface in $U_{\gamma-}$ with degree here defined by the normal projection of the vector field $\partial / \partial z$. The surface in question, $Z \cap U_{\gamma-}$, is embedded in $U_{\gamma-}$ and has the following four properties: First, it is agrees with $\Sigma \cap U_{\gamma-}$ where $s \geq-4 R_{*}$. Second, the 
$s \leq-2 R_{*}$ portion sits where $|z|<\rho_{*} / 100$. Third, the $s \leq-6 R_{*}$ portion is the union of $m_{\gamma-}$ disjoint, cylindrical ends. Finally, any given end, $\mathcal{E}$, is a graph of the form $(w, t) \rightarrow z_{\mathcal{E}} e^{-\varepsilon w}$ where $\varepsilon>0$ and where $z_{\mathcal{E}} \neq 0$. This description of the far right term in (3-47) follows directly from: The geometric interpretations of the far right term in (3-35) as the degree of the normal bundle of the surface in (3-36); the interpretation of the far right term in (3-45) as the degree of the normal bundle of the surface in (3-43); and the interpretation of the far right term in (3-46) as given at the end of Step 4.

Here is an analogous formula for $\sum_{1 \leq k \leq N_{\gamma+}} \operatorname{dim}\left(V_{\gamma+, k}\right)$ when $(\gamma, m) \in \Theta_{+}$:

$$
\sum_{1 \leq k \leq N_{\gamma+}} \operatorname{dim}\left(V_{\gamma+, k}\right)=\sum_{1 \leq q \leq m_{\gamma+}} z_{\gamma, q}-\sum_{\mathcal{E} \in \mathcal{E}_{\gamma+}} z_{\mathcal{E}}+\operatorname{deg}\left(N_{Z \cap U_{\gamma-}}\right) .
$$

The derivation of (3-48) is much the same as that of (3-47) and left to the reader. Add the various versions of (3-47), (3-48) and (3-29) to obtain an expression for $\operatorname{dim}\left(V_{0}\right)$. Given that $-\left\langle c_{1}, \Sigma\right\rangle=-\sum_{C \in \mathcal{C}}\left\langle c_{1}, C\right\rangle=-\left\langle c_{1}, Z\right\rangle$, the result is identical to what appears on the right hand side of (1-4). Note that this equivalence of first Chern class pairings follows from the fact that $Z$ differs from $\Sigma$ only on $\bigcup_{\gamma \in \Xi_{\Sigma-}} U_{\gamma-}$ and $\bigcup_{\gamma \in \Xi_{\Sigma+}} U_{\gamma+}$ where the section whose algebraic zero count computes $\left\langle c_{1}, \cdot\right\rangle$ is nonzero.

\section{3.b Instanton signs}

The purpose of this section is to prove the assertion made by the second bullet in Theorem 1.2. What follows directly sets the stage.

To start, remark that any given version of $\mathcal{M}_{1}\left(\Theta_{-}, \Theta_{+}\right)$has an orientation sheaf, $\Lambda\left(\Theta_{-}, \Theta_{+}\right)$, which is defined using Quillen's ideas about determinant line bundles. Definition 9.9 in [8] introduces the notion of a coherent system of orientations for

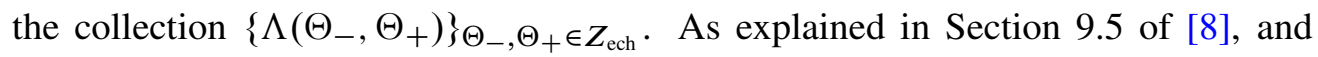
demonstrated below explicitly, a coherent system of orientations is specified by choosing an orientation for a certain real line that is associated to each hyperbolic Reeb orbit. A given coherent system of orientations orients any given version of $\mathcal{M}_{1}\left(\Theta_{-}, \Theta_{+}\right)$. This orientation is denoted by $o_{\text {ech }}$ in Theorem 1.2.

By way of reminder, Part 6 in Section 1.b introduced the 2-element set $\Lambda\left(\mathfrak{c}_{-}, \mathfrak{c}_{+}\right)$for any ordered pair $\mathfrak{c}_{-}, \mathfrak{c}_{+} \in \mathcal{M}^{r *}$. An element in this set canonically orients each component of $\mathcal{M}_{1}\left(\mathfrak{c}_{-}, \mathfrak{c}_{+}\right)$whose instantons are nondegenerate. As noted in Part 6 of Section $1 . b$, the set $\Lambda\left(\mathfrak{c}_{-}, \mathfrak{c}_{+}\right)$is canonically isomorphic as a $\mathbb{Z} / 2 \mathbb{Z}$ module to $\Lambda\left(\mathfrak{c}_{-}\right) \otimes_{\mathbb{Z} / 2 \mathbb{Z}}$ $\Lambda\left(\mathfrak{c}_{+}\right)^{*}$ where any given version of $\Lambda(\mathfrak{c})$ is a certain 2 -element set viewed as a nontrivial $\mathbb{Z} / 2 \mathbb{Z}$ module. A collection of elements $\left\{\mathfrak{o}\left(\mathfrak{c}_{-}, \mathfrak{c}_{+}\right) \in \Lambda\left(\mathfrak{c}_{-}, \mathfrak{c}_{+}\right)\right\}_{\mathfrak{c}_{-}, \mathfrak{c}_{+} \in \mathcal{M}^{r *}}$ 
was said to be coherent if there is a corresponding set $\{\mathfrak{o}(\mathfrak{c}) \in \Lambda(\mathfrak{c})\}_{\mathfrak{c} \in \mathcal{M}^{r *}}$ such that any given $\mathfrak{o}\left(\mathfrak{c}_{-}, \mathfrak{c}_{+}\right)$is $\mathfrak{o}\left(\mathfrak{c}_{-}\right) \mathfrak{o}\left(\mathfrak{c}_{+}\right)$.

The second bullet in Theorem 1.2 asserts that it is possible to choose elements $\{\mathfrak{o}(\mathfrak{c}) \in$

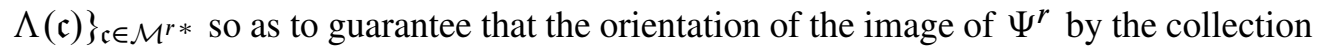

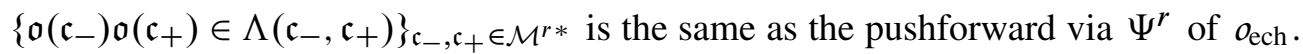

The proof that follows of this assertion has fourteen parts. The first two parts of the proof summarize material about determinant line bundles for families of Fredholm operators. This is relevant by virtue of the fact that the sheafs $\Lambda\left(\Theta_{-}, \Theta_{+}\right)$and $\Lambda\left(\mathfrak{c}_{-}, \mathfrak{c}_{+}\right)$are the orientation sheaves of such line bundles. Parts 3-5 introduce the notions that are used in

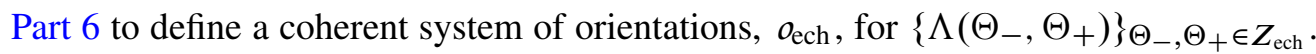
As is seen below, this requires as input a choice of orientation for a line that is associated to each hyperbolic Reeb orbit. Parts 7-13 use this same data to define an element $\mathfrak{o}\left(\mathfrak{c}_{-}, \mathfrak{c}_{+}\right)$, for $\Lambda\left(\mathfrak{c}_{-}, \mathfrak{c}_{+}\right)$when $\mathfrak{c}_{-}$and $\mathfrak{c}_{+}$are in the image of some large $r$ version of $\Phi^{r}$. The proof of the second bullet of the theorem is completed in Part 14 which explains why the $\mathfrak{o}\left(\mathfrak{c}_{-}, \mathfrak{c}_{+}\right)$orientation of $\mathcal{M}_{1}\left(\mathfrak{c}_{-}, \mathfrak{c}_{+}\right)$agrees on the image of $\Psi^{r}$ with the latter's pushforward of $o_{\text {ech }}$. Part 14 also proves that the set $\left\{\mathfrak{o}\left(\mathfrak{c}_{-}, \mathfrak{c}_{+}\right)\right\}_{\mathfrak{c}_{-}, \mathfrak{c}_{+} \in \operatorname{im}\left(\Phi^{r}\right)}$ is coherent in the sense defined above.

Part 1 As noted above, the sheaves $\Lambda\left(\Theta_{-}, \Theta_{+}\right)$and $\Lambda\left(\mathfrak{c}_{-}, \mathfrak{c}_{+}\right)$are the orientation sheaves of determinant line bundles. Quillen [11] introduced the notion of determinant line bundle for a family of Fredholm operators; and what follows gives a brief description. Much of what is said here in the context of embedded contact homology can be found in Section 9 of Hutchings and Taubes [8] and also in Bourgeois and Mohnke [2]. The corresponding story in the Seiberg-Witten context can be found in Chapter 20 of Kronheimer and Mrowka [9]. (See also Bismut and Freed [1] for more about the general subject of determinant line bundles.)

To start, let $\mathbb{L}_{0}$ and $\mathbb{L}_{1}$ denote separable Hilbert spaces, let $\mathcal{S}$ denote a space, and suppose that $s \rightarrow \Delta_{s}: \mathbb{L}_{0} \rightarrow \mathbb{L}_{1}$ denotes a continuous family of bounded, Fredholm operators parameterized by the points in $\mathcal{S}$. Now let $V_{1} \rightarrow \mathcal{S}$ denote a finite dimensional vector bundle, and $\phi_{1}: V_{1} \rightarrow \mathbb{L}_{1}$ denote an injective map with the following property: For all $s \in \mathcal{S}$, the composition of first $\phi_{1}$ and then projection to $\operatorname{cokernel}\left(\Delta_{\mathcal{S}}\right)$ is surjective onto cokernel $\left(\Delta_{s}\right)$. Under this assumption, the spaces $\left.V_{0}\right|_{s}=\left\{u \in \mathbb{L}_{0}\right.$ : $\left.\Delta_{\mathcal{S}}(u) \in \phi_{1}\left(\left.V_{1}\right|_{s}\right)\right\}$ define a vector bundle, $V_{0}$, over $\mathcal{S}$. The determinant line bundle for the family $s \rightarrow \Delta_{s}$ is the real line bundle with fiber

$$
\Lambda^{\text {top }}\left(\left.V_{0}\right|_{s}\right) \otimes \Lambda^{\text {top }}\left(\left.V_{1}\right|_{s}\right)^{*}
$$

Here, convention takes $\Lambda^{\text {top }}$ of the zero dimensional bundle to be $\mathbb{R}$. One can show that this bundle is independent of the choice of $V_{1}$ and $\phi_{1}$. This line bundle is denoted 
by $\operatorname{det}(\Delta)$. Note that there is an exact sequence

$$
0 \rightarrow \operatorname{kernel}\left(\Delta_{s}\right) \rightarrow V_{0} \rightarrow V_{1} \rightarrow \operatorname{cokernel}\left(\Delta_{s}\right) \rightarrow 0,
$$

where the middle arrow is the map $\Delta_{s}$ with it understood that $\phi_{1}$ is used to identify $V_{1}$ with $\phi_{1}\left(V_{1}\right)$. Thus, the line $\left.\operatorname{det}(\Delta)\right|_{s}$ is canonically isomorphic to

$$
\Lambda^{\text {top }}\left(\operatorname{kernel}\left(\Delta_{S}\right) \otimes \Lambda^{\text {top }}\left(\operatorname{cokernel}\left(\Delta_{s}\right)\right)^{*} .\right.
$$

The latter observation is relevant to the case at hand by virtue of the fact that an orientation for $\Sigma$ 's component of the moduli space $\mathcal{M}_{1}\left(\Theta_{-}, \Theta_{+}\right)$is no more nor less than an orientation for the product of the top exterior powers of operators that are associated to the components of $\Sigma$. To elaborate, suppose that $C \subset \Sigma$ is not an $\mathbb{R}$-invariant cylinder. Then the tangent space to $C$ 's component in $\mathcal{M}_{1}\left(\Theta_{-}, \Theta_{+}\right)$is canonically isomorphic to the kernel of the operator $\mathcal{D}_{C}$ as given in (1-10) but viewed as a Fredholm operator from $L_{1}^{2}(C ; N)$ to $L^{2}\left(C ; N \otimes T^{0,1} C\right)$. Suppose next that $C$ is an $\mathbb{R}$ invariant cylinder. Let $m$ denote the associated integer and let $C_{m} \rightarrow C$ denote the associated, $m$-fold connected cover. For the purposes of this proof, define $\mathcal{D}_{C}$ in this case to be the pullback to $C_{m}$ of the operator depicted in (1-10). Given (1-14) and the constraints in Section 4.b of [14], this version of $\mathcal{D}_{C}$ is a Fredholm operator from $L_{1}^{2}\left(C_{m} ; N\right)$ to $L^{2}\left(C_{m} ; N\right)$ with trivial kernel and cokernel. The point of all this is that the sheaf $\Lambda\left(\Theta_{-}, \Theta_{+}\right)$at $\Sigma$ is canonically isomorphic to the $\mathbb{Z} / 2 \mathbb{Z}$ module of orientations of the line

$$
\operatorname{det}\left(\bigoplus_{C \in \mathcal{C}} \mathcal{D}_{\mathcal{C}}\right)
$$

There is a similar story for the $\mathbb{Z} / 2 \mathbb{Z}$ module $\Lambda\left(\mathfrak{c}_{-}, \mathfrak{c}_{+}\right)$at $\mathfrak{d}_{\Sigma}$. The latter is canonically isomorphic to the $\mathbb{Z} / 2 \mathbb{Z}$ module of orientations for the line $\operatorname{det}(\mathfrak{D})$ where $\mathfrak{D}$ is $\mathfrak{d}_{\Sigma}$ 's version of the operator that is described in (1-12).

What follows is meant to give some indication of how the argument for the second point in Theorem 1.2 uses these determinant lines. It is first argued that a coherent orientation for (3-52) is determined in a suitably canonical fashion from an orientation of a tensor product of 3 lines, det- $\otimes \operatorname{det} \otimes \otimes \operatorname{det}_{+}{ }^{*}$, where each is the determinant line bundle for a Fredholm operator. The Fredholm operator that defines det_ is a version of $\mathcal{D}_{c}$ that is determined solely by $\Theta_{-}$. Furthermore, det- is oriented given the following data: First, a $\mathbb{Z} / 2 \mathbb{Z}$ choice for each hyperbolic Reeb orbit with even rotation number from $\Theta_{-}$. Second, an ordering of these orbits. Meanwhile, the Fredholm operator that defines det + is a version of $\mathcal{D}_{c}$ that is determined solely by $\Theta_{+}$; and det $t_{+}$is oriented given the $\Theta_{+}$analog of this same data. Finally, $\operatorname{det}_{\diamond}$ has a canonical orientation by virtue of the fact that the operator that defines this determinant line, yet another version of $\mathcal{D}_{C}$, has a deformation through Fredholm operators to one that is $\mathbb{C}$-linear. 
To say more about an orientation for the line $\operatorname{det}(\mathfrak{D})$, consider first any pair $\mathfrak{c}_{-}$and $\mathfrak{c}_{+}$of nondegenerate elements in $\operatorname{Conn}(E) \times C^{\infty}(M ; \mathbb{S})$. Let $\mathfrak{d}: \mathbb{R} \rightarrow \operatorname{Conn}(E) \times C^{\infty}(M ; \mathbb{S})$ denote any given smooth map with $s \rightarrow-\infty$ limit equal to $\mathfrak{c}_{-}$and with $s \rightarrow \infty$ limit equal to $\mathfrak{c}_{+}$. An element in $\Lambda\left(\mathfrak{c}_{-}, \mathfrak{c}_{+}\right)$is determined in a canonical fashion from an orientation of the determinant line that is associated to $d$ 's version of the operator in (1-12). As it turns out, one can choose a map $\mathfrak{d}$ so that this same version of $\operatorname{det}(\mathfrak{D})$ can be written in a suitable canonical fashion as Det_ $\otimes$ Det $_{0} \otimes$ Det $_{+}{ }^{*}$ where each of Det_, Det + and Det $\diamond$ is the determinant line of a Fredholm operator that differs from (1-12) by a zero-th order term. Moreover, the respective operators that give Det_ and Det + are determined solely by $\mathfrak{c}_{-}$and $\mathfrak{c}_{+}$. Meanwhile, the operator that gives Det $\diamond$ is homotopic through Fredholm operators to a $\mathbb{C}$-linear operator. This gives Det $\diamond \mathrm{a}$ canonical orientation which is assumed henceforth.

The fact that an element in any given $\mathfrak{c}_{-}$and $\mathfrak{c}_{+}$version of $\Lambda\left(\mathfrak{c}_{-}, \mathfrak{c}_{+}\right)$is determined solely by orientations for Det_ and Det + underlies the assertion that there are ele-

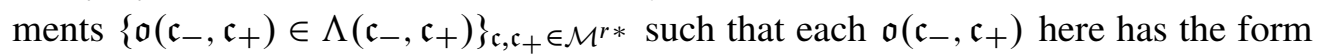

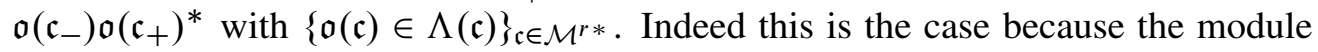
$\Lambda(\mathfrak{c})$ for $\mathfrak{c} \in \mathcal{M}^{r}$ is isomorphic to $\Lambda\left(\mathfrak{c}, \mathfrak{c}_{0}\right)$ with $\mathfrak{c}_{0}$ any given nondegenerate element in $\operatorname{Conn}(E) \times C^{\infty}(M ; \mathbb{S})$. To say more, define an element in $\Lambda\left(\mathfrak{c}_{-}, \mathfrak{c}_{0}\right)$ by orienting the version of $\operatorname{det}(\mathfrak{D})$ that is associated to a map $\mathfrak{d}: \mathbb{R} \rightarrow \operatorname{Conn}(E) \times C^{\infty}(M ; \mathbb{S})$ with $s \rightarrow-\infty$ limit is $\mathfrak{c}_{-}$and with $s \rightarrow \infty$ limit is $\mathfrak{c}_{0}$. As noted above, the associated version of $\operatorname{det}(\mathfrak{D})$ for such a map is canonically isomorphic to Det_ $\otimes$ Det$_{-} \diamond \otimes$ Det $_{0}$ where Det $\_\diamond$ and Det $_{0}$ are also determinant lines of Fredholm operators that differ from (1-12) by something of zero-th order. Moreover, the operator that defines Det $_{0}$ is determined solely by $\mathfrak{c}_{0}$, and the operator that defines Det_- $\diamond$ deformable through Fredholm operators to a $\mathbb{C}$-linear operator. This understood, fixing an orientation for Det $_{0}$ gives the element $\mathfrak{o}\left(\mathfrak{c}_{-}\right)$. There is an analogous $\mathfrak{o}\left(\mathfrak{c}_{+}\right)$. The composition law $\Lambda\left(\mathfrak{c}_{-}, \mathfrak{c}_{0}\right) \otimes_{\mathbb{Z} / 2 \mathbb{Z}} \Lambda\left(\mathfrak{c}_{0}, \mathfrak{c}_{+}\right)^{*}=\Lambda\left(\mathfrak{c}_{-}, \mathfrak{c}_{+}\right)$gives the latter the element $\mathfrak{o}\left(\mathfrak{c}_{-}\right) \mathfrak{o}\left(\mathfrak{c}_{+}\right)^{*}$.

It is important in what follows to note that the element $\mathfrak{o}\left(\mathfrak{c}_{-}\right) \mathfrak{o}\left(\mathfrak{c}_{+}\right)^{*}$ is the same as that determined for $\Lambda\left(\mathfrak{c}_{-}, \mathfrak{c}_{+}\right)$by the orientation of Det $-\otimes \operatorname{Det}_{\diamond} \otimes \operatorname{Det}_{+}{ }^{*}$ given previously. This is because the lines Det $\diamond$, Det_ $\diamond$ and the $\mathfrak{c}_{+}$analog of Det- $\diamond$ are all oriented by deforming their corresponding operators through Fredholm operators to something $\mathbb{C}$-linear.

With the preceding understood, let $\Sigma \in \mathcal{M}_{1}\left(\Theta_{-}, \Theta_{+}\right)$and take $\mathfrak{c}_{-}$and $\mathfrak{c}_{+}$to be respective solutions to (1-8) that determine the gauge equivalence classes of $\Phi^{r}\left(\Theta_{-}\right)$ and $\Phi^{r}\left(\Theta_{+}\right)$. A crucial observation made in what follows is that the associated tensor product Det_ $\otimes \operatorname{Det}_{\diamond} \otimes$ Det $_{+}$can be defined using $\Psi^{r}$ to supply the operator whose determinant line is Det_. Moreover, this version of Det_ is canonically isomorphic to the line det_ that is used to define the coherent orientation for $\Sigma$ 's version of (3-52). By 
the same token, $\Psi^{r}$ supplies a version of Det + that is canonically isomorphic to det $_{+}$. Finally, all of this is compatible with the pushforward via $\Psi^{r}$. These observations have the following sought after consequence: A coherent orientation for $\mathcal{M}_{1}\left(\Theta_{-}, \Theta_{+}\right)$ defines an element in $\Lambda\left(\mathfrak{c}_{-}, \mathfrak{c}_{+}\right)$which gives the same orientation to $\operatorname{det}(\mathfrak{D})$ as does the pushforward via $\Psi^{r}$. Moreover this element comes from a coherent orientation of

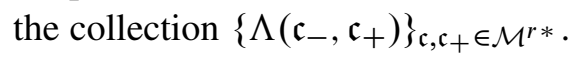

Part 2 This part of the proof states a well known gluing principle for first order Fredholm differential operators such as $\mathcal{D}_{C}$ and $\mathfrak{D}_{\mathfrak{d}}$. This gluing principle lies behind the composition law $\Lambda\left(\Theta_{-}, \Theta^{\prime}\right) \times_{\mathbb{Z} / 2 \mathbb{Z}} \Lambda\left(\Theta^{\prime}, \Theta_{+}\right)=\Lambda\left(\Theta_{-}, \Theta_{+}\right)$implied by Lemma 9.6 in [8]; and also the composition law $\Lambda\left(\mathfrak{c}_{-}, \mathfrak{c}_{0}\right) \times_{\mathbb{Z} / 2 \mathbb{Z}} \Lambda\left(\mathfrak{c}_{0}, \mathfrak{c}_{+}\right)=\Lambda\left(\mathfrak{c}_{-}, \mathfrak{c}_{+}\right)$that is stated in Chapter 20.3 of [9]. See also Section 9.6 in [8] or Corollaries 6 and 7 in [2] in the former case; and see (20-8) and the surrounding discussion in [9] or Mrowka's thesis [10] for the latter. In particular, this gluing principle is used momentarily to write triple product decompositions of both the determinant line bundle in (3-50) and of $\operatorname{det}(\mathfrak{D})$, this in the manner described at the end of Part 1.

What follows states the gluing principle in somewhat greater generality than is necessary. To set the stage, suppose that $Y$ is a smooth, Riemannian manifold, that $P_{0}$ and $P_{1}$ are smooth vector bundles over $Y$ with fiber metrics, and that

$$
\text { OO : } L_{1}^{2}\left(Y ; P_{0}\right) \rightarrow L^{2}\left(Y ; P_{1}\right)
$$

is a Fredholm, first order differential operator. Let $\phi: N \rightarrow Y$ denote an embedding of a separating, codimension 1 submanifold. Let $L: C^{\infty}\left(N ; \phi * P_{0}\right) \rightarrow C^{\infty}\left(N ; \phi * P_{1}\right)$ denote a first order, unbounded, self-adjoint elliptic differential operator with trivial kernel. Here, the metric on $N$ is that induced by $\phi$. Suppose that $\varepsilon>0$ has been specified, this a number that is much smaller than the smallest of the absolute values of the eigenvalues of $L$.

Suppose that $T \geq 2$ and that $\phi$ extends to an isometric embedding $\phi_{T}:[-T, T] \times N \rightarrow Y$ such that the following is true: Let $\pi:[-T, T] \times N \rightarrow N$ denote the projection. Then there are bundle isometries from $\phi_{T}^{*} P_{0,1}$ to $\pi^{*} \phi^{*} P_{0,1}$ that identify $\mathbb{O}$ on the image of $\phi_{T}$ with the operator

$$
\frac{\partial}{\partial s}+L+\mathfrak{e}
$$

on $[-T, T] \times N$. Here, $\mathfrak{e}$ is a first order operator whose coefficients are such that their derivatives to order 3 are bounded by $\varepsilon$.

To continue, define manifolds $Y_{-}$and $Y_{+}$as follows: The manifold $Y_{-}$is obtained by discarding the part of $Y$ that contains the $\phi$-image of $(1, T] \times N$ and replacing the 
latter with $(1, \infty) \times N$. Meanwhile, $Y_{+}$is obtained by discarding from $Y$ the part that contains the $\phi$-image of $[-T,-1) \times N$ and replacing the latter with $(-\infty,-1) \times N$. Both $P_{0}$ and $P_{1}$ extend in the obvious fashion to $Y_{-}$and $Y_{+}$. Likewise, the operator $(\mathbb{O}$ extends over both $Y_{-}$and $Y_{+}$so as to map $L_{1}^{2}\left(Y_{ \pm}, P_{0}\right)$ to $L^{2}\left(Y_{ \pm}, P_{1}\right)$. The latter operators are denoted by $\mathbb{O}_{ \pm}$. Indeed, $\mathbb{O}_{-}=\partial / \partial s+L+\chi \mathfrak{e}$ on the $(1, \infty) \times N$ part of $Y_{-}$; meanwhile $\mathbb{O}_{+}=\partial / \partial s+L+(1-\chi) \mathfrak{e}$ on the $(-\infty,-1) \times N$ part of $Y_{+}$. Finally, define maps $\phi_{-}: C^{\infty}\left(Y_{-}, P_{0,1}\right) \rightarrow C^{\infty}\left(Y, P_{1}\right)$ by sending $\phi_{-}(\eta)=\chi \eta$. Likewise, define $\phi_{+}: C^{\infty}\left(Y_{-}, P_{0,1}\right) \rightarrow C^{\infty}\left(Y, P_{1}\right)$ by $\phi_{+}(\eta)=(1-\chi) \eta$.

The lemma that follows states the promised gluing principle. This lemma uses $\mathbb{O}_{ \pm}^{\dagger}$ and $\mathbb{O}^{\dagger}$ to denote the formal $L^{2}$ adjoints of the indicated operators.

Lemma 3.4 There exists $\varepsilon_{*}>0$ and $T_{*} \geq 2$ with the following significance: If $\varepsilon<\varepsilon_{*}$ and $T \geq T_{*}$, then

(a) The operators $\mathbb{O}_{ \pm}$define Fredholm maps from $L_{1}^{2}\left(Y_{ \pm}, P_{0}\right)$ to $L^{2}\left(Y_{ \pm}, P_{1}\right)$, respectively.

(b) The composition of first $\phi_{-} \oplus \phi_{+}$and then $L^{2}$ orthogonal projection onto the kernel of $\Delta^{\dagger}$ defines a surjection $\phi_{1}: \operatorname{kernel}\left(\mathbb{O}_{-}^{\dagger}\right) \oplus \operatorname{kernel}\left(\mathbb{O}_{+}^{\dagger}\right) \rightarrow \operatorname{kernel}\left(\mathbb{O}^{\dagger}\right)$.

(c) The composition of $\phi_{-} \oplus \phi_{+}$and then $L_{1}^{2}$ orthogonal projection onto $\operatorname{kernel}(\Delta)$ is an isomorphism from $\operatorname{kernel}\left(\mathbb{O}_{-}\right) \oplus \operatorname{kernel}\left(\mathbb{O}_{+}\right)$to $V_{0}=\left\{u \in L_{1}^{2}\left(Y ; P_{0}\right)\right.$ : OO $\left.u \in \operatorname{Image}\left(\phi_{1}\right)\right\}$.

(d) In particular, $\phi_{-} \oplus \phi_{+}$induces and isomorphism of lines

$\Lambda^{\text {top }}\left(\operatorname{kernel}\left(\mathbb{O}_{-}\right) \oplus \operatorname{kernel}\left(\mathbb{O}_{+}\right)\right) \otimes \Lambda^{\text {top }}\left(\operatorname{cokernel}\left(\mathbb{O}_{-}\right) \oplus \operatorname{cokernel}\left(\mathbb{O}_{+}\right)\right)^{*}$ $\approx \operatorname{det}(\mathbb{O})$.

A proof is straightforward to come by; indeed, the arguments are much like those that are used to below to prove Lemma 3.5. See also Lemma 9.6 in [8] which gives a relevant $N=S^{1}$ version of (3-55). Various $N=M$ versions have been used since the early days of gauge theory (see, eg Chapter 7 in [3]). Very much more sophisticated versions in the Seiberg-Witten context are used in Chapters 17 and 24 of [9].

An $N=S^{1}$ version of (3-55) gives the composition law $\Lambda\left(\Theta_{-}, \Theta^{\prime}\right) \times_{\mathbb{Z} / 2 \mathbb{Z}} \Lambda\left(\Theta^{\prime}, \Theta_{+}\right)=$ $\Lambda\left(\Theta_{-}, \Theta_{+}\right)$. A version for $N=M$ gives the composition law $\Lambda\left(\mathfrak{c}_{-}, \mathfrak{c}_{0}\right) \times_{\mathbb{Z} / 2 \mathbb{Z}}$ $\Lambda\left(\mathfrak{c}_{0}, \mathfrak{c}_{+}\right)=\Lambda\left(\mathfrak{c}_{-}, \mathfrak{c}_{+}\right)$given in Chapter 20 of [9].

Part 3 This part of the proof serves as a digression to introduce some auxiliary operators that are used in Part 6 to define a canonical orientation for the line in (3-52).

These operators are used to define the lines det_, det+ and $\operatorname{det} \diamond$ that are mentioned in Part 1. 
To start the digression, fix $\varepsilon \in(0,1)$ but very small, fix $k \in \mathbb{Z}$, and define the operator $\mathcal{D}_{0-}$ on $C^{\infty}\left(\mathbb{R} \times S^{1} ; \mathbb{C}\right)$ by declaring it to send any given function $\eta$ to

$$
\mathcal{D}_{0-} \eta=\frac{1}{2}\left(\frac{\partial}{\partial w}+i \frac{\partial}{\partial t}+\left(\frac{1}{2} k+\varepsilon(1-\chi)\right)\right) \eta+i \varepsilon \chi e^{i k t} \bar{\eta}
$$

where $\chi=\chi(w)$. This operator is Fredholm from $L_{1}^{2}\left(\mathbb{R} \times S^{1} ; \mathbb{C}\right)$ to $L^{2}\left(\mathbb{R} \times S^{1} ; \mathbb{C}\right)$. A spectral flow calculation can be done to prove that it has index 0 when $k$ is odd and index 1 when $k$ is even. Moreover, if $\varepsilon$ is small, its kernel dimension is, respectively, 0 and 1 . Indeed, when $k$ is even, the kernel is the real span of $(1-i) e e^{u(w)+i k t / 2}$ where the function $u$ is an antiderivative of $\varepsilon(1-2 \chi)$. Fix such a small value of $\varepsilon$. In the case when $k$ is even, fix an orientation for the kernel of $\mathcal{D}_{0-}$. If $T \geq 0$ is given, use $\mathcal{D}_{T-}$ to denote the operator that is obtained from $\mathcal{D}_{0-}$ by translating the latter using $w \rightarrow w+T$. Thus $\mathcal{D}_{T-}$ is obtained from (3-56) by replacing $\chi$ with the function $\chi_{T-}=\chi(w+T)$.

Consider next the operator $\mathcal{D}_{0+}$ on $C^{\infty}\left(\mathbb{R} \times S^{1} ; \mathbb{C}\right)$ that sends a given function $\eta$ to

$$
D_{0+} \eta=\frac{1}{2}\left(\frac{\partial}{\partial w}+i \frac{\partial}{\partial t}+\frac{1}{2}(k+\varepsilon \chi)\right) \eta+i \varepsilon(1-\chi) e^{i k t} \bar{\eta} .
$$

This operator is also Fredholm from $L_{1}^{2}\left(\mathbb{R} \times S^{1} ; \mathbb{C}\right)$ to $L^{2}\left(\mathbb{R} \times S^{1} ; \mathbb{C}\right)$. The operator $\mathcal{D}_{0+}$ has index zero when $k$ is odd and index -1 when $k$ is even. If $\varepsilon>0$ is small, it has trivial kernel and cokernel when $k$ is odd, and trivial kernel but 1-dimensional cokernel when $k$ is even. Fix such a small value for $\varepsilon$, and then fix an orientation for the cokernel of $\mathcal{D}_{0+}$ when $k$ is even. Given $T \geq 0$, define $\mathcal{D}_{T+}$ by replacing $\chi$ in (3-57) by the function $\chi_{T+}=\chi(w-T)$.

There are yet two more operators that play a roles in what follows. Both map $\mathbb{C}^{\infty}\left(\mathbb{R} \times S^{1} ; \mathbb{C}\right)$ to itself. The first has the form

$$
\eta \rightarrow \mathcal{D}_{-} \eta=\frac{1}{2}\left(\frac{\partial}{\partial w}+i \frac{\partial}{\partial t}+\frac{1}{2}(k+\varepsilon)\right) \eta .
$$

Note that this operator is $\mathbb{C}$-linear. Note as well that it agrees with $\mathcal{D}_{T-}$ at points in $\mathbb{R} \times S^{1}$ where $-T<w$. The second operator of the two operators is denoted by $\mathcal{D}_{+}$; and it is defined by replacing $\varepsilon$ with $-\varepsilon$ in (3-58). Note that $\mathcal{D}_{+}$is also $\mathbb{C}$-linear, and that it agrees with $\mathcal{D}_{T+}$ where $T<w$.

Part 4 This part of the proof describes a deformation of certain $C \in \mathcal{C}$ versions of $\mathcal{D}_{C}$. The particulars are motivated by what is said in Section 9 of [8]. To set the stage, suppose that $\gamma \in \Xi_{\Sigma-}$ is a hyperbolic Reeb orbit and let $\mathcal{E}$ denote the end of $\Sigma$ that lies in $U_{\gamma-}$. Use $C$ in what follows to denote the component of $\Sigma$ that contains $\mathcal{E}$. 
View $\mathcal{E}$ as a graph $(w, t) \rightarrow z_{\mathcal{E}}(w, t)$ as in (4-2) of [14], and then identify the normal bundle to $\mathcal{E}$ with $\mathbb{C}$ using the projection of $\partial / \partial z$. This done, the operator $\mathcal{D}_{C}$ appears on $\mathcal{E}$ as

$$
\eta \rightarrow \mathcal{D}_{C} \eta=\frac{1}{2}\left(\frac{\partial}{\partial w}+i \frac{\partial}{\partial t}+\frac{1}{2} k\right) \eta+i \varepsilon e^{i k t} \bar{\eta}+\mathfrak{e}(\eta)
$$

where $|\mathfrak{e}| \leq c_{0}(|\eta|+|\nabla \eta|) e^{-|w| / c_{0}}$ Here, $k$ is the rotation number of $\gamma$.

Fix $T>4 R$ and $T^{\prime}>8 T$; and consider now the operator on $\mathcal{E}$ that sends a function $\eta$ to the operator

$$
\eta \rightarrow \chi_{T-} \mathcal{D}_{T^{\prime}-}+\left(1-\chi_{T-}\right) \mathcal{D}_{C}
$$

The deformation of $\mathcal{D}_{C}$ that is depicted in (3-60) is homotopic to $\mathcal{D}_{C}$ through a path of Fredholm, first order operators. Indeed, such a homotopy parametrized by $[0,1]$ can be obtained by replacing $\chi_{T-}$ in (3-60) by $\sigma \chi_{T-}$ where $\sigma \in[0,1]$.

Let $\gamma \in \Xi_{\Sigma+}$ denote a hyperbolic Reeb orbit, let $\mathcal{E}$ denote the end of $\Sigma$ in $U_{\gamma+}$, and let $C$ denote the component of $\Sigma$ that contains $\mathcal{E}$. There is a deformation of $\mathcal{D}_{C}$ through Fredholm operators that is the analog of (3-60): Replace $\chi_{T-}$ with $\left(1-\chi_{T+}\right)$ and replace $\mathcal{D}_{T^{\prime}-}$ with $\mathcal{D}_{T^{\prime}+}$.

Given $C \in \mathcal{C}$, use $\mathcal{D}_{C ; T, T}^{\prime}$ to denote the operator on $C$ that is obtained by replacing $\mathcal{D}_{C}$ by the appropriate version of (3-60) or its positive end counterpart on each end $\mathcal{E} \subset C$ whose constant $s$ slices limit as $|s| \rightarrow \infty$ to a hyperbolic Reeb orbit. It follows from what is said above that this operator is homotopic to $\mathcal{D}_{C}$ via a path of Fredholm, first order differential operators acting from $L_{1}^{2}(C ; N)$ to $L^{2}\left(C ; N \otimes T^{0,1} C\right)$. As just noted, such a homotopy, parametrized by $\sigma \in[0,1]$, is obtained by replacing each occurrence of $\chi_{T \pm}$ by $\sigma \chi_{T \pm}$.

Fix $T>4 R$ and $T^{\prime}>8 T$ so as to introduce the line

$$
\operatorname{det}\left(\bigoplus_{C \in \mathcal{C}} \operatorname{det}\left(\mathcal{D}_{C ; T, T^{\prime}}\right)\right)
$$

Here, as in (3-52), the operator associated to an $\mathbb{R}$-invariant cylinder component $C$ that comes with weight $m>1$ is that defined by the pullback of $\mathcal{D}_{C}$ to the $m$-fold covering cylinder. Cylinders of this sort have $\mathcal{D}_{C ; T, T^{\prime}}=\mathcal{D}_{C}$ as they are of the form $\mathbb{R} \times \gamma$ with $\gamma$ an elliptic Reeb orbit.

Since each version of $\mathcal{D}_{C ; T, T}$, is homotopic through Fredholm operators to $\mathcal{D}_{C}$, an orientation for the line in (3-61) defines one for the line in (3-52); thus it defines a point in the $\mathbb{Z} / 2 \mathbb{Z}$ module $\Lambda\left(\Theta_{-}, \Theta_{+}\right)$at $\Sigma$. 
Part 5 The two operators $\mathcal{D}_{ \pm}$are now used to define yet another operator that is associated to a given component $C \subset \Sigma$. This operator, $\mathcal{D}_{C \diamond}$, is defined as follows: First $\mathcal{D}_{C} \diamond$ differs from $\mathcal{D}_{C}$ only on those ends of $C$ whose constant $s$ slices converge as $|s| \rightarrow \infty$ to a hyperbolic Reeb orbit. This understood, let $\mathcal{E}$ denote an end of $C$ whose constant $s$ slices converge as $s \rightarrow-\infty$ to a hyperbolic Reeb orbit. Then $\mathcal{D}_{C} \diamond$ on $\mathcal{E}$ sends a complex-valued function $\eta$ to

$$
\mathcal{D}_{C \diamond} \eta=\chi_{T-} \mathcal{D}_{-}+\left(1-\chi_{T-}\right) \mathcal{D}_{C} .
$$

If $\mathcal{E} \subset C$ is an end whose constant $s$ slices converge as $s \rightarrow \infty$ to a hyperbolic Reeb orbit, then $\mathcal{D}_{C} \diamond$ on $\mathcal{E}$ sends $\eta$ to $\mathcal{D}_{C \diamond} \eta=\left(1-\chi_{T+}\right) D_{+}+\chi_{T+} \mathcal{D}_{C}$.

The operator $\mathcal{D}_{C \diamond}$ is also Fredholm as a map from $L_{1}^{2}(C ; N)$ to $L^{2}\left(C ; T^{0,1} C\right)$. Even so, it is not homotopic to $C$ through Fredholm operators unless $C$ has no ends whose constant $s$ slices converge as $|s| \rightarrow \infty$ to a hyperbolic Reeb orbit with even rotation number. Indeed, let $n_{C-}$ denote the number of such ends that lie where $s<-1$ and let $n_{C+}$ denote the number that lie where $s>1$. Then

$$
\operatorname{index}\left(\mathcal{D}_{C \diamond}\right)=\operatorname{index}\left(\mathcal{D}_{C}\right)-n_{C-}+n_{C+} .
$$

This operator $\mathcal{D}_{C} \diamond$ has the nice property that it is $\mathbb{C}$-linear at large values of $|s|$. As a consequence, it is homotopic through a family of Fredholm, first order differential operators mapping $L_{1}^{2}(C ; N)$ to $L^{2}\left(C ; N \otimes T^{0,1} C\right)$ to a $\mathbb{C}$-linear operator. For example, a homotopy parametrized by $[0,1]$ is obtained by writing $\mathcal{D}_{C} \diamond$ as

$$
\eta \rightarrow D_{C \diamond} \eta=\bar{\partial} \eta_{c}+v_{C} \diamond \eta_{c}+\mu_{C} \diamond \bar{\eta}_{c}
$$

and then taking the $\sigma \in[0,1]$ operator in the homotopy to be what is obtained by replacing $\mu_{C \diamond}$ in (3-64) by $(1-\sigma) \mu_{C} \diamond$.

Here is why the existence of such a homotopy is relevant: The determinant line bundle of a $\mathbb{C}$-linear operator has a canonical orientation, this defined by taking the vector space $V_{1}$ in (3-49) to be complex. As a consequence, $\operatorname{det}\left(\mathcal{D}_{C} \diamond\right)$ also has a canonical orientation. This orientation is deemed the positive orientation.

By the way, the existence of such a homotopy implies that $\mathcal{D}_{C} \diamond$ has even index.

Part 6 Let $n_{-}$denote the number of hyperbolic Reeb orbits with even rotation number that come from $\Theta_{-}$, and let $n_{+}$denote the analogous number from $\Theta_{+}$. If $T>4 R$ and $T^{\prime} \gg T$, then it follows from Lemma 3.4 that $\operatorname{det}\left(\mathcal{D}_{C ; T, T^{\prime}}\right)$ is canonically isomorphic to

$$
\operatorname{det}\left(\bigoplus_{C \in \mathcal{C}} \mathcal{D}_{C ; T, T^{\prime}}\right)=\Lambda^{\text {top }}\left(\bigoplus_{n_{-}} \mathbb{R}\right) \otimes \operatorname{det}\left(\bigoplus_{C \in \mathcal{C}} \mathcal{D}_{C \diamond}\right) \otimes \Lambda^{\text {top }}\left(\bigoplus_{n_{+}} \mathbb{R}\right)
$$


Here, each factor of $\mathbb{R}$ that appears in the first term on the right in (3-65) accounts for the 1 -dimensional kernel of one of the $n_{-}$versions of $\mathcal{D}_{T^{\prime}-}$. Likewise, each factor of $\mathbb{R}$ that appears in the far right hand term accounts for the 1-dimensional cokernel of one of the versions of $\mathcal{D}_{T^{\prime}+}$. just noted, $\operatorname{det}\left(\mathcal{D}_{C} \diamond\right)$ has a canonical orientation, and for the same reason, so does $\operatorname{det}\left(\bigoplus_{C \in \mathcal{C}} \mathcal{D}_{C \diamond}\right)$. Note in this regard that the ordering of the components of $\Sigma$ has no bearing on this orientation as each version of $\mathcal{D}_{C} \diamond$ has even index.

With the preceding understood, it follows from (3-65) that $\operatorname{det}\left(\bigoplus_{C \in \mathcal{C}} \mathcal{D}_{C ; T, T^{\prime}}\right)$ has a canonical orientation given two additional choices. Here is the first choice: Each hyperbolic Reeb orbit with even rotation number has its associated operator $\mathcal{D}_{0-}$. An orientation is required for the kernel of each such version of $\mathcal{D}_{0-}$. Note that such a choice induces an orientation on the given Reeb orbit's version of $\mathcal{D}_{0+}$. Indeed, this follows from Lemma 3.4 with the following observation: Take $T \ll-1$ and define $\mathcal{D}_{T 0}=(1-\chi) \mathcal{D}_{T_{-}}+\chi \mathcal{D}_{T_{+}}$. This operator $\mathcal{D}_{T 0}$ is Fredholm and it is homotopic through Fredholm operators to the $\mathbb{C}$-linear operator $\mathcal{D}_{-}$given in (3-58).

The second choice required is that of an ordering for the respective subsets of $\Xi_{\Sigma_{-}}$and of $\Xi_{\Sigma_{+}}$that consist of hyperbolic orbits with even rotation number. With such orderings chosen, the resulting orientation for $\operatorname{det}\left(\bigoplus_{C \in \mathcal{C}} \mathcal{D}_{C ; T, T^{\prime}}\right)$ induces an orientation for the line in (3-52).

Now let $\Sigma$ and $\Sigma^{\prime}$ define distinct components of $\mathcal{M}_{1}\left(\Theta_{-}, \Theta_{+}\right)$. It is a consequence of what is said in Section 9 of [8] that the orientation and $\Sigma^{\prime}$ versions of (3-52) define the same element of the $\mathbb{Z} / 2 \mathbb{Z}$ module $\Lambda\left(\Theta_{-}, \Theta_{+}\right)$; and it follows that this element comes from a system of coherent orientations as described in Definition 9.9 of [8]. This element in $\Lambda\left(\Theta_{-}, \Theta_{+}\right)$is deemed the positive element. It is the element $o_{\text {ech }}$ in Theorem 1.2.

Part 7 Fix $\Sigma \subset \mathcal{M}_{1}\left(\Theta_{-}, \Theta_{+}\right)$, and reintroduce the operator $\mathfrak{D}_{\Sigma}$ as defined in Part 1 of Section 3.a. What follows here and in Parts 8-10 use constructions from Section 3.a to relate the kernel and cokernel of an operator such as $\mathfrak{D}_{\Sigma}$ with certain vector spaces of sections along the components of $\Sigma$. The general sort of operator that is considered in what follows is denoted by $\mathfrak{D}_{\Sigma *}$ and this Part 7 gives its definition. The notation used in this definition and subsequently is the same as that introduced in Section 3.a.

To set the stage, suppose that $\gamma$ is a hyperbolic Reeb orbit from $\Xi_{\Sigma_{-}}$. Fix a pair of complex functions $\left(v_{\gamma-*}, \mu_{\gamma-*}\right): \mathbb{R} \times S^{1} \rightarrow \mathbb{C}$ with the following properties: First $v_{\gamma-*}$ is real where $w \ll-1$ and both are independent of $w \in \mathbb{R}$ for $w \ll-1$. Second, the latter $w$-independent pair defines a version of (1-2) with trivial kernel. Fix an analogous pair, $\left(v_{\gamma+*}, \mu_{\gamma+*}\right): \mathbb{R} \times S^{1} \rightarrow \mathbb{C}$ for each hyperbolic Reeb orbit $\gamma \in \Xi_{\Sigma_{+}}$ Such that $v_{\gamma+*}$ is real where $w \gg 1$, both are independent of $w$ where $w \gg 1$, and 
is such that the version of (1-2) defined by the latter $w$-independent pair has trivial kernel.

To continue, suppose that $C \subset \mathcal{C}$ is not of the form $\mathbb{R} \times \gamma$ where $\gamma$ is an elliptic Reeb orbit. Fix for $C$ a pair $\left(v_{C *}, \mu_{C *}\right)$ with $\nu_{C *}$ being a section of $T^{0,1} C$ and $\mu_{C *}$ being section of $N^{2} \otimes T^{0,1} C$. Constrain $\nu_{C *}$ and $\mu_{C *}$ on the ends of $C$ as follows: If $\gamma \in \Xi_{\Sigma_{ \pm}}$is an elliptic Reeb orbit and $C \cap U_{\gamma \pm} \neq \varnothing$, then $\nu_{C *}=v_{C}$ and $\mu_{C *}=\mu_{C}$ on $C \cap U_{\gamma \pm}$. If $\gamma \in \Xi_{\Sigma_{ \pm}}$is a hyperbolic Reeb orbit and $C \cap U_{\gamma \pm} \neq \varnothing$, first identify $C$ 's end $\mathcal{E}$ in $U_{\gamma \pm}$ as a graph via (4-2) in [14] and trivialize $\mathcal{E}$ 's normal bundle using the orthogonal projection along $C$ of $\partial / \partial z$. This identifies $\left(v_{C *}, \mu_{C *}\right)$ as a pair of functions on $(-\infty,-R] \times S^{1}$ or on $[R, \infty) \times S^{1}$ as the case may be. Granted this identification require that $\left.\left(v_{C *}, \mu_{C *}\right)\right|_{(w, t)}=\left.\left(v_{\gamma \pm *}, \mu_{\gamma \pm *}\right)\right|_{t}+\mathfrak{e}$, where $\mathfrak{e}$ and its derivatives to third order are bounded by $c_{0} e^{-2 \lambda_{q_{\mathcal{E}}} w}$ Here, $\lambda_{q_{\mathcal{E}}}$ is the eigenvalue that appears in $\mathcal{E}$ 's version of (4-2) in [14].

To end the stage setting, reintroduce the operators $\mathfrak{D}$ and $\mathfrak{D}_{\xi}$ as described in Part 1 of Section 3.a. Use (3-1) to define $\mathfrak{e}_{q *}$.

The operator $\mathfrak{D}_{\Sigma *}$ is written as $\mathfrak{D}_{\Sigma *}=\mathfrak{D}_{\xi *}+r^{1 / 2} \mathfrak{e}_{q *}(\cdot)$ where $\mathfrak{D}_{\xi *}$ is the first order, elliptic operator from $L_{1}^{2}\left(\mathbb{R} \times M ; i T^{*} M \oplus S \oplus i \mathbb{R}\right)$ to $L^{2}\left(\mathbb{R} \times M ; i T^{*} M \oplus S \oplus i \mathbb{R}\right)$ that is described in the five steps that follow.

Step 1 This step and the next three steps define an operator $\mathfrak{P}_{\xi *}$ with respective domain range spaces $L_{1}^{2}\left(\mathbb{R} \times M ; i T^{*} M \oplus S \oplus i \mathbb{R}\right)$ and $L^{2}\left(\mathbb{R} \times M ; i T^{*} M \oplus S \oplus i \mathbb{R}\right)$. To start the definition, take $\mathfrak{P}_{\xi *}=\mathfrak{D}_{\xi}$ at points with distance $\rho_{\Sigma}$ or greater from $\Sigma$.

Step 2 Recall that $\mathcal{C}$ denotes the set of components of $\Sigma$. Fix $C \in \mathcal{C}$ and at points in $U_{C}$, write $\mathfrak{P}_{\xi *}=\chi_{c} \mathfrak{P}_{c *}+\left(1-\chi_{c}\right) \mathfrak{D}_{\xi}$, where $\mathfrak{P}_{c *}$ is obtained from $\mathfrak{D}_{\xi}$ by making two replacements. To describe the first, write $\mathfrak{D}_{\xi}$ on $U_{C}$ as in (6-6) and (6-7) of [14] with the refinement of (6-6) in [14] that is given in (2-78). Now replace $C$ 's version of $(\nu, \mu)$ in (2-78) by $\left(v_{C *}, \mu_{C *}\right)$. To describe the second, recall from Part 1 of Section 3.a that the pair $\left(A^{\xi}, \psi^{\xi}\right)$ that defines $\mathfrak{D}_{\xi}$ has a contribution from $\mathfrak{h}(\xi)$, this as described in Section 6.d of [14]. The component $C$ contributes to $\mathfrak{h}(\xi)$ via $\mathfrak{b}_{C}$ in (6-36) of [14] with $\mathfrak{b}_{C}$ given as a section, $\left(0, \mathfrak{p}_{C}\right)$ of $\mathbb{V}_{c 0} \oplus \mathbb{V}_{C 1}$. Here, $\mathfrak{p}_{C}$ is defined by (6-33) of [14]. All this understood, the second replacement that defines $\mathfrak{P}_{c *}$ is obtained by replacing $\mathfrak{p}_{c}$ in the definition of $\mathfrak{h}(\xi)$ with the solution, $\mathfrak{p}_{c *}$, to $-i \theta_{C, r}^{\dagger} \mathfrak{p}_{\mathbb{C} *}-\left(1-\Pi^{0}\right) \mathfrak{v}_{C *}=0$ where $\mathfrak{v}_{C *}$ is defined by using $v_{C *}$ and $\mu_{C *}$ in the $\xi=0$ version of (6-32) of [14] instead of $v_{C}$ and $\mu_{C}$.

Step 3 The operator $\mathfrak{P}_{\xi *}$ is equal to $\mathfrak{D}_{\xi}$ in any $U_{\gamma \pm}$ when $\gamma \in \Xi_{\Sigma_{ \pm}}$is an elliptic Reeb orbit. Note that this is consistent with (3-65) because if $\gamma$ is elliptic, then 
$\left(v_{C *}, \mu_{C *}\right)-\left(v_{C}, \mu_{C}\right)$ at points in $U_{C} \cap U_{\gamma \pm}$, and therefore $\mathfrak{P}_{C *}=\mathfrak{D}_{C}$ at points in $U_{C} \cap U_{\gamma \pm}$.

Step 4 Take $\gamma \in \Xi_{\Sigma_{ \pm}}$to be a hyperbolic Reeb orbit such that $\Sigma \cap U_{\gamma \pm} \neq \varnothing$. Let $\mathcal{E}$ denote the end of $\Sigma$ in $U_{\gamma \pm}$ and let $C$ denote the component of $\Sigma$ that contains $\mathcal{E}$. Write $\mathfrak{P}_{\xi *}$ in $U_{\gamma \pm}$ at distances less than $\rho_{\Sigma}$ from $\mathcal{E}$ as $\mathfrak{P}_{\xi *}=\chi_{C}\left(\chi_{\mathcal{E}} \mathfrak{P}_{\gamma \pm *}+\right.$ $\left.\left(1-\chi_{\mathcal{E}}\right) \mathfrak{P}_{C *}\right)+\left(1-\chi_{C}\right) \mathfrak{D}_{\xi}$ where $\mathfrak{P}_{\gamma \pm *}$ is obtained from $\mathfrak{D}_{\xi}$ by making two replacements. These are the analogs of those made in Step 2. To elaborate, write $\mathfrak{D}_{\xi}$ on $U_{\gamma \pm}$ as described in Part 4 of Section 6.a of [14] using the relevant version of (6-6) and (6-7) of [14] with the refinement of (6-6) given by the $U_{\gamma \pm}$ version of (2-78). Then replace $\gamma$ 's version of $(\nu, \mu)$ in (2-78) by the pair $\left(v_{\gamma \pm *}, \mu_{\gamma \pm *}\right)$. This is the first replacement. To describe the second, recall from Part 1 of Section 3.a that the pair $\left(A^{\xi}, \psi^{\xi}\right)$ that defines $\mathfrak{D}_{\xi}$ has a contribution from $\mathfrak{h}(\xi)$ as described in Section 6.d of [14]. If $\gamma \in \Xi_{\Sigma_{-}}$, then it contributes to $\mathfrak{h}(\xi)$ via $\mathfrak{b}_{\gamma-}$ in (6-43) and (6-44) in [14]. If $\gamma \in \Xi_{\Sigma_{+}}$, it contributes via the analogous $\mathfrak{b}_{\gamma+}$ using the $U_{\gamma+}$ analogs of (6-43) and (6-44) in [14]. In any event, $\mathfrak{b}_{\gamma \pm}$ is given as a section, $\left(0, \mathfrak{p}_{\gamma \pm}\right)$ of $\mathbb{V}_{0} \oplus \mathbb{V}_{1}$. Here, $\mathfrak{p}_{\gamma \pm}$ is defined by in Part 5 of Section 6.d in [14]. The second replacement that defines $\mathfrak{P}_{\gamma \pm *}$ is obtained by using $\left(v_{\gamma \pm *}, \mu_{\gamma \pm *}\right)$ instead of $\gamma$ 's version of $(\nu, \mu)$ when defining $\mathfrak{p}_{\gamma \pm}$ as instructed in Part 5 of Section 6.d in [14].

Step 5 Note that $\mathfrak{P}_{\xi *}$ and $\mathfrak{D}_{\xi}$ have the same first order symbol. As a consequence, $\mathfrak{P}_{\xi *}$ can be written as $\nabla_{s}+\mathfrak{L}_{\xi_{*}}$, where $\mathfrak{L}_{\xi_{*}}$ contains only derivatives along the $M$ factor in $\mathbb{R} \times M$. This understood, set $\mathfrak{D}_{\xi^{*}}=\nabla_{s}+\frac{1}{2}\left(\mathfrak{L}_{\xi_{*}}+\mathfrak{L}_{\xi *}^{t}\right)$ where $\mathfrak{L}_{\xi *}^{t}$ here denotes the formal, $L^{2}$-adjoint. Note that $\mathfrak{D}_{\xi *}=\mathfrak{D}_{\xi}$ when all versions of $\left(v_{(\cdot) *}, \mu_{(\cdot) *}\right)$ are equal to the original, unstarred version. This is the case because $\mathfrak{D}_{\xi}=\nabla_{s}+\mathfrak{L}_{\xi}$ and $\mathfrak{L}_{\xi}$ is symmetric. The fact that $\mathfrak{L}_{\xi *}$ is not, in general, symmetric is a consequence of the definition in Part 3 of Section 6.a in [14] of what is denoted by $q$ in $(2-78)$.

Here is a key point to keep in mind in what follows: Let $C \in \mathcal{C}$ or $\gamma \in U_{\gamma \pm}$. Then the corresponding version of (6-6) and (6-7) in [14] with the refinement of (2-78) holds for $\mathfrak{D}_{\xi *}$ with it understood that $\left(v_{C *}, \mu_{C *}\right)$ is used in lieu of $\left(v_{C}, \mu_{C}\right)$, or $\left(v_{\gamma \pm *}, \mu_{\gamma \pm *}\right)$ is to be used in lieu $\gamma$ 's version of $(\nu, \mu)$, as the case may be.

Part 8 Fix data $\left\{\left(v_{C *}, \mu_{C *}\right)\right\}_{C \in \mathcal{C}}$ as described above so as to construct the operator $\mathfrak{D}_{\Sigma *}$. What follows summarizes some of the salient features of this operator. Note that it is to be understood below that what is written as $\mathcal{D}_{C *}$ when $C=\mathbb{R} \times \gamma$ has associated integer $m>1$ denotes the pullback of $\mathcal{D}_{C}$ to the nontrivial, $m$-fold cover of $C$. The next two lemmas refers to the parameter $R$ that is used in the construction of $\Psi^{r}$. This parameter is defined in Section 4.c of [14]. 
Lemma 3.5 Fix $Q \geq 1$. Then there exists $\kappa \geq 1$ such that the following is true: Take $r \geq \kappa^{2}, R \geq \kappa$; and suppose that each $C \in \mathcal{C}$ version of $\left(v_{C *}, \mu_{C *}\right)$ and each $\gamma \in \Xi_{\Sigma_{ \pm}}$version of $\left(v_{\gamma \pm}, \mu_{\gamma \pm}\right)$ has $C^{3}$-norm bounded by $Q$. The operator $\mathfrak{D}_{\Sigma *}: L_{1}^{2}\left(\mathbb{R} \times M ; i T^{*} M \oplus S \oplus i \mathbb{R}\right) \rightarrow L^{2}\left(\mathbb{R} \times M ; i T^{*} M \oplus S \oplus i \mathbb{R}\right)$ is Fredholm with index equal to $\Sigma_{C \in \mathcal{C}}$ index $\left(\mathcal{D}_{C *}\right)$. In addition, there exists a canonical isomorphism $\operatorname{det}\left(\mathfrak{D}_{\Sigma *}\right) \approx \operatorname{det}\left(\bigoplus_{C \in \mathcal{C}} \mathcal{D}_{C *}\right)$.

The next lemma is a slight refinement to Lemma 3.5.

Lemma 3.6 Suppose either that all $C \in \mathcal{C}$ versions of $\mathcal{D}_{C *}$ have trivial cokernel, or that all have trivial kernel. Then there exists $\kappa \geq 1$ with the following significance: If $r \geq \kappa^{2}$ and $R \geq \kappa$, then there are isomorphisms $\operatorname{kernel}\left(\mathfrak{D}_{\xi *}\right) \approx \operatorname{kernel}\left(\bigoplus_{C \in \mathcal{C}} \mathcal{D}_{C *}\right)$ and cokernel $\left(\mathfrak{D}_{\Sigma *}\right) \approx \operatorname{cokernel}\left(\bigoplus_{C \in \mathcal{C}} \mathcal{D}_{C *}\right)$ that are compatible with the determinant line isomorphism of Lemma 3.5.

Note in particular that Lemma 3.6 is applicable in the case that $\left\{\mathcal{D}_{C *}=\mathcal{D}_{C}\right\}_{C \in \mathcal{C}}$ and so when $\mathfrak{D}_{\Sigma *}=\mathfrak{D}_{\Sigma}$. In this case, all cokernels are trivial, and there exists exactly one $C \in \mathcal{C}$ where the kernel of $\mathcal{D}_{C}$ is not trivial. In this case, the kernel is 1 -dimensional and a nonzero element can be taken to be the generator at $\Sigma$ of the $\mathbb{R}$ action on $\mathcal{M}_{1}\left(\Theta_{-}, \Theta_{+}\right)$that is induced by the translations along the $\mathbb{R}$ factor of $\mathbb{R} \times M$. The isomorphism takes this element to the element in the kernel of $\mathfrak{D}_{\Sigma}$ that gives the generator at $\mathfrak{d}_{\Sigma}$ of the corresponding $\mathbb{R}$ action on $\mathcal{M}_{1}\left(\mathfrak{c}_{-}, \mathfrak{c}_{+}\right)$. The fact that the respective $\mathbb{R}$-action generators are mapped to each other follows from the fact that the constructions are done in an $\mathbb{R}$-equivariant fashion.

The final result is a corollary to Lemma 3.6 that concerns the $\mathbb{R}$-invariant case. To set the stage, suppose that $\Theta_{-}=\Theta_{+}$and that each $C \in \mathcal{C}$ is an $\mathbb{R}$-invariant cylinder. Assume that the instanton $\mathfrak{d}_{\Sigma}$ is independent of the $\mathbb{R}$ factor on $\mathbb{R} \times M$. This is to say that $\mathfrak{d}_{\Sigma}=(A, \psi)$ is constant map to $\operatorname{Conn}(E) \times C^{\infty}(M ; \mathbb{S})$ with $(A, \psi)$ the pair that is constructed using the data $\Theta_{-}=\Theta_{+}$as instructed in Section 3 of [14]. Finally, assume that each $C \in \mathcal{C}$ version of $\left(v_{C *}, \mu_{C *}\right)$ is $\mathbb{R}$-invariant. In this case, the operator $\mathfrak{D}_{\Sigma *}$ is also $\mathbb{R}$-invariant, and it can be written as

$$
\mathfrak{D}_{\Sigma *}=\frac{\partial}{\partial s}+\mathfrak{L}_{*},
$$

where $\mathfrak{L}_{*}$ differs from what is written in (1-9) by a zero-th order endomorphism. The operator $\mathfrak{L}_{*}$ is symmetric, and self-adjoint with domain $L_{1}^{2}\left(M ; i T^{*} M \oplus S \oplus i \mathbb{R}\right)$.

Corollary 3.7 There exists $\kappa \geq 1$ such that if $r \geq \kappa$, then the operator $\mathfrak{L}_{*}$ has trivial kernel.

The proofs of Lemmas 3.5 and 3.6 are contained in Part 9. 
Part 9 To set the stage for the proofs, suppose that $Q \geq 1$ and suppose in what follows that each $C \in \mathcal{C}$ version of $\left(v_{C *}, \mu_{C *}\right)$ and each $\gamma \in \Xi_{\Sigma_{-}}$version $\Sigma \pm$ of $\left\{\left(v_{\gamma \pm}, \mu_{\gamma \pm}\right)\right.$ has $C^{3}$-norm bounded by $Q$.

The operator $\mathfrak{D}_{\xi *}$ has a decomposition as in (3-11), this written as

$$
\begin{aligned}
\left(1-\Pi_{\xi}\right) \mathfrak{D}_{\xi *} \mathfrak{f} & =\mathfrak{D}_{\xi *}^{\perp} \mathfrak{f}^{\perp}(\Theta)+p_{*}(\theta), \\
t^{\dagger}\left(\mathfrak{D}_{\xi} \mathfrak{f}\right) & =p_{*}{ }^{\prime}\left(\mathfrak{f}^{\perp}\right)+\Delta_{*} \theta .
\end{aligned}
$$

The fact that (6-6) and (6-7) of [14] still hold has the following implication: The analysis that led to (3-12)-(3-13) can be repeated to obtain a constant, $c_{Q}>1$, such that

$$
\begin{aligned}
& \left\|\mathfrak{D}_{\xi *}{ }^{\perp} \mathfrak{f}^{\perp}\right\|_{2} \geq c_{Q}\left\|\mathfrak{f}^{\perp}\right\|_{\mathbb{H}}, \\
& \begin{aligned}
&\left\|p_{*}(\theta)\right\|_{2} \leq c_{Q} r^{3 \sigma}\|\theta\|_{\mathcal{K}_{1}^{2}}, \\
&\left\|p_{*}{ }^{\prime}\left(\mathfrak{f}^{\perp}\right)\right\|_{\mathcal{L}^{2}} \leq c_{Q} r^{-1 / 2+3 \sigma}\left\|\mathfrak{f}^{\perp}\right\|_{\mathbb{H}},
\end{aligned}
\end{aligned}
$$

when $R \geq c_{Q}$ and $r \geq c_{Q}^{2}$.

The next lemma says what is needed about $\Delta_{*}$. The constant $R$ that appears in the lemma is from Section 4.c of [14].

Lemma 3.8 Fix $Q \geq 1$ and there exists $\kappa>1$ with the following significance: Suppose that each $C \in \mathcal{C}$ version of $\left(v_{C *}, \mu_{C *}\right)$ and each $\gamma \in \Xi_{\Sigma_{ \pm}}$version of $\left\{\left(v_{\gamma_{ \pm}}, \mu_{\gamma_{ \pm}}\right)\right.$has $C^{3}$-norm bounded by $Q$. For each such $C \in \mathcal{C}$ not of the form $\mathbb{R} \times \gamma$ with $\gamma$ an elliptic Reeb orbit, there exist vector spaces $V_{1 C} \subset L^{2}\left(C ; N \otimes T^{0,1} C\right)$ and $V_{0 C} \subset L_{1}^{2}(C ; N)$ such that

- $L^{2}$ projection from $V_{C 1}$ to $\operatorname{kernel}\left(\mathcal{D}_{C *}\right)$ is surjective.

- $L_{1}^{2}$-orthogonal projection from $V_{C 0}$ onto the subspace $\left\{u \in L_{1}^{2}(C ; N): \mathcal{D}_{C *} u \in\right.$ $\left.V_{1 C}\right\}$ is an isomorphism.

- Let $V_{0}=\bigoplus_{C \in \mathcal{C}} V_{C 0}$ and $V_{1}=\bigoplus_{C \in \mathcal{C}} V_{C 1}$. If $r \geq \kappa^{2}$ and if $R \geq \kappa$, there exist monomorphisms $\phi_{1}: V_{1} \rightarrow \mathcal{L}^{2}$ and $\phi_{0}: V_{0} \rightarrow \mathcal{K}_{1}^{2}$ such that

(a) The projection from $\phi_{1}\left(V_{1}\right)$ to the cokernel of $\Delta_{*}$ is surjective

(b) The composition of $\phi_{0}$ followed by the $\mathcal{K}_{1}^{2}$-orthogonal projection to the vector space $\left\{\theta \in \mathcal{K}_{1}^{2}: \Delta_{*} \theta \in \phi_{1}\left(V_{1}\right)\right\}$ defines an isomorphism, $\phi_{0 *}$, that obeys $\left\|\phi_{0}-\phi_{0 *}\right\|_{\mathcal{K}_{1}^{2}} \leq \frac{1}{4} \kappa R^{-1}\left\|\phi_{0}\right\|_{\mathcal{K}_{1}^{2}}$

(c) Let $\mathbb{P}: \mathcal{L}^{2} \rightarrow \mathcal{L}^{2}$ denote the $\mathcal{L}^{2}$ projection orthogonal to $\phi_{1}\left(V_{1}\right)$. Then $\left\|\mathbb{P} \Delta_{*} \lambda\right\|_{L^{2}} \geq \kappa^{-1}\|\lambda\|_{\mathcal{K}_{1}^{2}}$ when $\lambda$ is $\mathcal{K}^{2}$ orthogonal to $\left\{\theta \in \mathcal{K}_{1}^{2}: \Delta_{*} \theta \in\right.$ $\left.\phi_{1}\left(V_{1}\right)\right\}$.

This lemma is proved in Part 10. Accept it as true until then. 
The proof of Lemma 3.5 requires, in general, both $V_{0}$ and $V_{1}$ to be nontrivial. A proof of Lemma 3.6 is obtained from the arguments below by taking either $V_{1}=0$ and $V_{0}=\bigoplus_{C \in \mathcal{D}} \operatorname{kernel}\left(\mathcal{D}_{\mathbb{C} *}\right)$ or else $V_{0}=0$ and $V_{1}=\bigoplus_{C \in \mathcal{C}} \operatorname{cokernel}\left(\mathcal{D}_{\mathbb{C} *}\right)$ as the case may be.

Proof of Lemma 3.5 The proof has three steps.

Step 1 Fix $\mathfrak{y} \in \mathbb{L}$. It follows by virtue of (3-5), (3-11) and (3-67) and (3-68) that a given $\mathfrak{f}=\mathfrak{f}^{\perp}+t(\theta) \in \mathbb{H}$ obeys $\mathfrak{D}_{\Sigma *} \mathfrak{f}=\mathfrak{y}$ if and only if $\theta$ obeys an equation that has the schematic form

$$
\Delta_{*} \theta+e_{*}(\theta)=t^{\dagger}(\mathfrak{y})-p_{*}^{\prime}\left(\left(\mathfrak{D}_{\Sigma *}{ }^{\perp}\right)^{-1}\left(1-\Pi_{\xi}\right) \mathfrak{y}\right),
$$

where $\mathfrak{D}_{\Sigma *}^{\perp} \mathfrak{l}^{\perp}$ is defined to be $\mathfrak{D}_{\xi *}^{\perp} \mathfrak{l}^{\perp}+r^{1 / 2}\left(1-\Pi_{\xi}\right)\left(\mathfrak{e}_{q} *^{\perp} \mathfrak{l}^{\perp}\right)$; and where $e_{*}$ is the analog of what is denoted by $e$ in (3-16). This $e_{*}$ obeys the following analog of (3-17):

$$
\left\|e_{*}(\theta)\right\|_{\mathcal{L}^{2}} \leq c_{0} r^{-1 / 2+8 \sigma}\|\theta\|_{\mathcal{K}_{1}^{2}}
$$

It follows from (3-69) that $\operatorname{kernel}\left(\mathfrak{D}_{\Sigma}\right)$ is canonically isomorphic to the kernel of $\Delta_{*}+e_{*}$ and that the cokernel of $\mathfrak{D}_{\Sigma}$ is canonically isomorphic to the cokernel of $\Delta_{*}+e_{*}$.

Step 2 It follows from the isomorphisms just described that the operator $\mathfrak{D}_{\Sigma^{*}}$ is Fredholm if and only if $\Delta_{*}+e_{*}$ has finite dimensional kernel and cokernel. This understood, it is sufficient to prove the following:

- The $\mathcal{L}^{2}$ projection of $\phi_{1}\left(V_{1}\right)$ to the cokernel of $\Delta_{*}+e_{*}$ is surjective.

- The $\mathcal{K}_{1}^{2}$-orthogonal projection of $\phi_{0}\left(V_{0}\right)$ onto $\left\{\theta \in \mathcal{K}_{1}^{2}:\left(\Delta_{*}+e_{*}\right) \theta \in\right.$ $\left.\phi_{1}\left(V_{1}\right)\right\}$ is an isomorphism.

To prove the first assertion, suppose that $u \in \operatorname{kernel}\left(\left(\Delta_{*}+e_{*}\right)^{\dagger}\right)$ is $\mathcal{L}^{2}$-orthogonal to $\phi_{1}\left(V_{1}\right)$. It follows from Part (c) of the second bullet in Lemma 3.8 that there exists a unique $\lambda \in \mathcal{K}_{1}^{2}$ such that $\lambda$ is $\mathcal{K}_{1}^{2}$-orthogonal to the space $\left\{\theta \in \mathcal{K}_{1}^{2}: \Delta_{*} \theta \in \phi_{1}\left(V_{1}\right)\right\}$ and such that $\mathbb{P} \Delta_{*} \lambda=u$. This understood, it follows that

$$
\left\langle u, \mathbb{P} \Delta_{*} \lambda\right\rangle_{\mathcal{L}^{2}}=\|u\|_{\mathcal{L}^{2}}^{2} .
$$

On the other hand the small size of $e_{*}$ implies that

$$
\left\langle u, \mathbb{P} \Delta_{*} \lambda\right\rangle \leq c_{0} r^{-1 / 2+8 \sigma}\|\lambda\|_{\mathcal{K}_{1}^{2}}\|u\|_{\mathcal{L}^{2}}
$$

and this is compatible with (3-72) only if $\|\lambda\|_{\mathcal{K}_{1}^{2}} \geq c_{0}{ }^{-1} r^{1 / 2-8 \sigma}\|u\|_{\mathcal{L}^{2}}$. This lower bound is not consistent with what is said in Part (c) of the second bullet in Lemma 3.8. 
What follows next explains why the $\mathcal{K}_{1}^{2}$-orthogonal projection maps $\phi_{0}\left(V_{0}\right)$ onto the vector space $\left\{\theta \in \mathcal{K}_{1}^{2}:\left(\Delta_{*}+e_{*}\right) \theta \in \phi_{1}\left(V_{1}\right)\right\}$ is an isomorphism. Suppose that $\theta$ is $\mathcal{K}_{1^{-}}^{2}$ orthogonal to $\phi_{0}\left(V_{0}\right)$ and is such that $\Delta_{*} \theta+e_{*}(\theta) \in \phi_{1}\left(V_{1}\right)$. Part (c) of the second bullet in Lemma 3.8 implies the following: There exists a unique $\lambda \in \mathcal{K}_{1}^{2}$ such that $\lambda$ is $\mathcal{K}_{1^{-}}^{2}$ orthogonal to the vector space $\left\{\theta \in \mathcal{K}_{1}^{2}: \Delta_{*} \theta \in \phi_{1}\left(V_{1}\right)\right\}$ and $\mathbb{P} \Delta_{*} \lambda=\mathbb{P} \Delta_{*} \theta$. Given the small size of $e$, it follows that $\|\lambda\|_{\mathcal{K}_{1}^{2}} \leq c_{0} r^{-1 / 2+8 \sigma}\|\theta\|_{\mathcal{K}_{1}^{2}}$ It then follows that $\Delta_{*}(\theta+\lambda) \in \phi_{1}\left(V_{1}\right)$ so there exists $u \in V_{0}$ such that $\phi_{0 *}(u)=\theta+\lambda$. Given the small size of $\lambda$, this runs afoul of Part (b) of the second bullet of Lemma 3.8 unless $\theta=0$.

Step 3 The identification described in Step 1 between the respective kernels of $\mathfrak{D}_{\Sigma^{*}}$ and $\Delta_{*}+e_{*}$ and between their respective cokernels induces a canonical isomorphism between $\operatorname{det}\left(\mathfrak{D}_{\Sigma^{*}}\right)$ and $\operatorname{det}\left(\Delta_{*}+e_{*}\right)$. Meanwhile, the homomorphisms that are described in (3-71) supply a canonical isomorphism between $\operatorname{det}\left(\Delta_{*}+e_{*}\right)$ and $\Lambda^{\text {top }}\left(V_{0}\right) \otimes \Lambda^{\text {top }}\left(V_{1}\right)$. The composition of these two isomorphisms gives the asserted isomorphisms between $\operatorname{det}\left(\mathfrak{D}_{\Sigma^{*}}\right)$ and $\Lambda^{\text {top }}\left(V_{0}\right) \otimes \Lambda^{\text {top }}\left(V_{1}\right)$.

Proof of Lemma 3.6 Suppose first that each $C \in \mathcal{C}$ version of $\mathcal{D}_{C *}$ has trivial cokernel. In this case the first bullet in (3-71) implies that the cokernel of $\mathfrak{D}_{\Sigma *}$ is trivial and the second bullet gives the lemma's asserted isomorphism between $V_{0}=$ $\bigoplus_{C \in \mathcal{C}} \operatorname{kernel}\left(\mathcal{D}_{C *}\right)$ and kernel of $\mathfrak{D}_{\Sigma *}$. The other possibility has $V_{0}=0$ and $V_{1}=$ $\bigoplus_{C \in \mathcal{C}} \operatorname{cokernel}\left(\mathcal{D}_{C *}\right)$. In this case, the second bullet in (3-71) implies that $\mathfrak{D}_{\Sigma *}$ has trivial kernel and that the projection of $\phi_{1}$ to the cokernel of $\Delta_{*}+e_{*}$ is injective. This and the first bullet of (3-71) imply that the composition of $\phi_{1}$ followed by projection defines an isomorphism from $V_{1}$ to the cokernel of $\mathfrak{D}_{\Sigma *}$. The latter is the isomorphism asserted by Lemma 3.6.

Part 10 This part of the proof contains:

Proof of Lemma 3.8 There are six steps to the proof.

Step 1 Given that (2-78) holds for $\mathfrak{D}_{C *}$ using $\left(v_{C *}, \mu_{C *}\right)$ in lieu of $\left(v_{C}, \mu_{C}\right)$, and using any $\gamma \in \Xi_{\Sigma_{ \pm}}$version of $\left(v_{\gamma \pm *}, \mu_{\gamma \pm *}\right)$ in lieu of $\gamma$ 's version of $(\nu, \mu)$ has the following consequence: What is done in Part 5 of Section 3.a can be repeated to obtain a description of $\Delta$ that is, but for two changes, given by the relevant versions of (3-20), (3-21), or by (3-22) and (3-23), or by their $\gamma \in \Xi_{\Sigma_{+}}$counterparts. The first change replaces $\left(v_{C}, \mu_{C}\right)$ in (3-20) with $\left(v_{C *}, \mu_{C *}\right)$. The second change replaces $(\nu, \mu)$ in (3-22) with $\left(v_{\gamma \pm *}, \mu_{\gamma \pm *}\right)$. 
Step 2 Let $C$ denote a component of $\Sigma$ that is not an $\mathbb{R}$-invariant cylinder; let $\gamma$ denote an elliptic Reeb orbit in either $\Xi_{\Sigma_{-}}$or $\Xi_{\Sigma_{+}}$, and suppose that $\mathcal{E} \subset C$ is an end that sits in $U_{\gamma_{-}}$or $U_{\gamma_{+}}$as the case may be. Since $\left(v_{C}, \mu_{C}\right)$ and $\left(v_{C *}, \mu_{C *}\right)$ agree here, there exists $c_{0} \geq 1$ such that any element $u \in \operatorname{kernel}\left(\mathcal{D}_{C *}\right)$ and any $u \in \operatorname{kernel}\left(\mathcal{D}_{C *}{ }^{\dagger}\right)$ must obey

$$
|u|+|\nabla u| \leq c_{0} e^{-(|w|-2 R) / c_{0}}\|u\|_{L^{2}}
$$

on $\mathcal{E}$. Suppose next that $\gamma$ is a hyperbolic Reeb orbit in either $\Xi_{\Sigma_{-}}$or $\Xi_{\Sigma_{+}}$, and that $\mathcal{E}$ is an end of $C$ that lies in $U_{\gamma_{-}}$or $U_{\gamma_{+}}$as the case may be. In this case, any $u$ from either the kernel of $\mathcal{D}_{C *}$ or its cokernel obeys

$$
|u|+|\nabla u| \leq c_{Q}\left(1+e^{\left(|w|-T^{\prime}-2 R\right) / c_{0}}\right)^{-1}\|u\|_{L^{2}}
$$

on $\mathcal{E}$. Here, $c_{Q}$ depends on the bound, $Q$, for the $C^{2}$ norms of $v_{c *}$ and $\mu_{c *}$. Meanwhile, $T^{\prime}$ is such that each $\gamma \in \Xi_{\Sigma-}$ version of $\left\{\left(v_{\gamma-}, \mu_{\gamma-}\right)\right.$ is constant for $w<-T^{\prime}$, and each $\gamma \in \Xi_{\Sigma_{+}}$version of $\left\{\left(v_{\gamma+}, \mu_{\gamma+}\right)\right.$ is constant for $w>T^{\prime}$.

Step 3 This step constitutes a digression to state and then prove a key lemma.

Lemma 3.9 Fix $Q \geq 1$. There exists $\kappa \geq 1$ with the following significance: Suppose that $C \subset \mathcal{C}$ is not of the form $\mathbb{R} \times \gamma$ with $\gamma$ and elliptic Reeb orbit. Assume that $\left(v_{c *}, \mu_{c *}\right)$ and each $\gamma \in \Xi_{\Sigma_{ \pm}}$version of $\left(v_{\gamma \pm *}, \mu_{\gamma \pm *}\right)$ is as described in Part 7 and has $C^{3}$ norm bounded by $Q$. Then there exists a finite dimensional subspace $V_{C 1} \subset L^{2}\left(C ; N \otimes T^{0,1} C\right)$ with the following properties:

- $L^{2}$-orthogonal projection maps $V_{1 C}$ onto kernel $\left(\mathcal{D}_{C *}{ }^{\dagger}\right)$.

- Let $V_{C 0}=\left\{u \in L_{1}^{2}(C ; N): \mathcal{D}_{C *} u \in V_{C 1}\right\}$. If $u \in L^{2}{ }_{1}(C ; N)$ and if $u$ is $L_{1}^{2}$-orthogonal to $V_{C 1}$, then $\left\|\mathcal{D}_{C *} u\right\|_{L^{2}} \geq \kappa^{-1}\|u\|_{L_{1}^{2}}$.

- If $u \in V_{C 1}$ or if $u \in V_{C 0}$, then (3-73) and (3-74) hold on the ends of $C$.

Proof of Lemma 3.9 In what follows, $c_{Q}$ in each appearance denotes a constant that depends only on $C$ and $Q$, but not otherwise on the particulars of $\left(v_{c *}, \mu_{c *}\right)$. As with $c_{0}$, the value of $c_{Q}$ can be assumed to increase between subsequent appearances.

Associate to each elliptic Reeb orbit in $\Xi_{\Sigma_{-}}$the smallest of the absolute values of the eigenvalues for the operator in (1-2) when acting on functions from $\mathbb{R}$ to $\mathbb{C}$ that are $2 \pi n$ periodic for $n \in\left\{1, \ldots, m_{\gamma-}\right\}$. Associate to each elliptic Reeb orbit in $\Xi_{\Sigma_{+}}$the analogous positive number. If $\gamma \in \Xi_{\Sigma_{-}}$is hyperbolic, associate instead the smallest of the absolute values of the eigenvalues of the version of (1-2) that is defined using the pair $\left.\lim _{s \rightarrow-\infty}\left(v_{\gamma-*}, \mu_{\gamma-*}\right)\right|_{s}$ in lieu of $\gamma$ 's version of $(\nu, \mu)$. Associate the analogous 
positive number to each hyperbolic $\gamma$ in $\Xi_{\Sigma+}$; here, the version of (1-2) is defined using the pair that is defined by the $s \rightarrow \infty$ limit of $\left.\left(v_{\gamma+*}, \mu_{\gamma+*}\right)\right|_{s}$. Each Reeb orbit now has an associated positive number. Set $\varepsilon_{0}$ to equal 1/100 times the smallest of these positive numbers.

Fix for the moment $\varepsilon \in\left(0, \varepsilon_{0}\right)$ and let $\mathcal{U} \subset L_{1}^{2}(C ; N)$ denote the set of elements in $L_{1}^{2}(C ; N)$ with unit $L^{2}$ norm and with the property $\left\|\mathcal{D}_{C *} u\right\|_{L^{2}} \leq \varepsilon$. Note that the Bochner-Weitzenboch formula for the operator $\mathcal{D}_{C *}$ implies that $\|u\|_{L_{1}^{2}} \leq c_{Q}$ when $u \in \mathcal{U}$.

To say more about the set $\mathcal{U}$, let $\gamma$ denote an elliptic Reeb orbit in either $\boldsymbol{\Xi}_{\Sigma_{-}}$or $\Xi_{\Sigma_{+}}$and suppose that $C \cap U_{\gamma-} \neq \varnothing$. Let $\hat{\chi}$ denote the function on $C$ with support on $C \cap U_{\gamma-}$ or $C \cap U_{\gamma+}$ as the case may be, where it equals $\chi((2 R-|w|) / R)$. As

$$
\varepsilon>\left\|\hat{\chi} D_{C *} u\right\|_{L^{2}} \geq\left\|\mathcal{D}_{C *}(\hat{\chi} u)\right\|_{L^{2}}-c_{0} R^{-1}\|u\|_{L^{2}} \geq \varepsilon_{0}\|\hat{\chi} u\|_{L^{2}}-c_{0} R^{-1},
$$

it follows that

$$
\int_{C} \hat{\chi}|u|^{2} \leq c_{0}\left(\varepsilon+R^{-1}\right)^{2} .
$$

If $\gamma \in \Xi_{ \pm}$is a hyperbolic orbit, then (3-76) holds if $\hat{\chi}$ is defined to be the function on $C$ with support in $C \cap U_{\gamma \pm}$ where it equals $\chi\left(\left(T^{\prime}+2 R-|w|\right) / R\right)$.

The existence of an upper bound for the $L_{1}^{2}$ norm of any $u \in \mathcal{U}$ implies that $\mathcal{U}$ is weakly compact in the $L_{1}^{2}$ topology. This plus (3-76) implies an additional $L^{2}$ compactness property whose description follows. To start, let $w$ denote the function on $C$ that is obtained by summing all of the $\gamma \in \Xi_{\Sigma_{-}}$and $\gamma \in \Xi_{\Sigma_{+}}$versions of what is denoted by $\hat{\chi}$ in (3-76). If $u \in \mathcal{U}$, then the function $(1-w) u$ has compact support and is such that

$$
\begin{aligned}
\|u-(1-w) u\|_{L^{2}} & \leq c_{Q}\left(\varepsilon+R^{-1}\right) \\
\left\|\mathcal{D}_{C *}((1-w) u)\right\|_{L^{2}} & \leq c_{Q}\left(\varepsilon+R^{-1}\right) \\
\|(1-w) u\|_{L_{1}^{2}} & \leq C_{Q} .
\end{aligned}
$$

As a consequence, the image of $\mathcal{U}$ under the map $u \rightarrow(1-w) u$ maps $\mathcal{U}$ in a bounded and injective fashion to a set of $L_{1}^{2}$ sections of $N$ with compact support where $|s| \leq\left(T^{\prime}+2 R\right)$. This image of $\mathcal{U}$ under this map is weakly compact in $L_{1}^{2}$ and, by virtue of the Relich lemma, strongly compact in $L^{2}$. In particular, there exists a finite set, $\widehat{U}_{\varepsilon} \subset L_{1}^{2}(C ; N)$, with the following property: If $u \in \mathcal{U}$, then $(1-w) u$ has $L^{2}$-norm less than $\varepsilon$ from an element in $\widehat{U}_{\varepsilon}$. It then follows from (3-77) that any $u \in \mathcal{U}$ has $L^{2}$ distance less than $c_{Q^{\varepsilon}}$ from an element in $\hat{U}_{\varepsilon}$. This then implies that 
there exists a finite set, $\mathcal{U}_{\varepsilon} \subset \mathcal{U}$ such that any $u \in \mathcal{U}$ has $L^{2}$ distance less than $c_{k} \varepsilon$ from an element in $(1-w) \mathcal{U}_{\varepsilon}$.

This last observation implies the following stronger assertion: Any $u \in \mathcal{U}$ has $L_{1}^{2}$ distance less than $c_{Q}\left(\varepsilon+R^{-1}\right)$ from some element in $(1-w) \mathcal{U}_{\varepsilon}$. To see why, first use the Bochner-Weitzenboch formula for $\mathcal{D}_{C *}$ to see that

$$
\left\|\nabla\left(u-u^{\prime}\right)\right\|_{L^{2}} \leq c_{Q}\left(\left\|D_{C *} u\right\|_{L^{2}}+\left\|\mathcal{D}_{C *} u^{\prime}\right\|_{L^{2}}+\left\|u-u^{\prime}\right\|_{L^{2}}\right)
$$

for any given pair $u$ and $u^{\prime}$ from $L_{1}^{2}(C ; N)$; and then use (3-77). Given that $\varepsilon+R^{-1}<c_{Q}{ }^{-1}$, the preceding assertion about the $L_{1}^{2}$ distance between points in $\mathcal{U}$ and $(1-w) \mathcal{U}_{\varepsilon}$ has the following corollary:

$$
\begin{aligned}
& \text { If } u \in L_{1}^{2}(C ; N) \text { is } L_{1}^{2} \text { orthogonal to all elements in }(1-w) \mathcal{U}_{\varepsilon} \text {, then } \\
& \left\|\mathcal{D}_{C *} u\right\|_{L^{2}} \geq \varepsilon\|u\|_{L_{1}^{2}} \text {. }
\end{aligned}
$$

With the preceding understood, define $V_{C 1} \subset L^{2}\left(C ; N \otimes T^{0,1} C\right)$ to equal the span of the set $\left\{\mathcal{D}_{C *} u+x: u \in(1-w) \mathcal{U}_{\varepsilon}\right.$ and $\left.x \in \operatorname{kernel}\left(\mathcal{D}_{C *}^{\dagger}\right)\right\}$. The assertion in Lemma 3.9's first bullet holds by construction. It follows from (3-79) that a $Q$-dependent choice of $\varepsilon$ can be made so that the assertion of Lemma 3.9's second bullet also holds with $\kappa$ depending only on $Q$. The third bullet of the lemma concerns points in $C$ where $v_{C *}=V_{C}$ and $\mu_{C *}=\mu_{C}$ and so where $\mathcal{D}_{C *}=\mathcal{D}_{C}$. As a consequence, the proof that these assertions hold for $w \in \operatorname{kernel}\left(\mathcal{D}_{C *}^{\dagger}\right)$ is now standard; see Hofer, Wysocki and Zehnder [5] or Lemma 2.6 and Section 3 in Hutchings and Taubes [8]. Meanwhile, the assertion also holds for the elements $\mathcal{D}_{C *}((1-w) u)$ with $u \in \mathcal{U}_{\varepsilon}$ because the latter vanishes on the relevant portions of $C$. The assertion made by the third bullet for $u \in V_{0 C}$ follows using the arguments used in [5] or those used in Sections 2 and 3 of [8] because an element $u \in V_{0 C}$ obeys $\mathcal{D}_{c} u=0$ on a neighborhood of the relevant portions of $C$.

Step 4 But for one modification, the map $\phi_{0}$ is constructed by mimicking what is done in Step 1 of the proof of Lemma 2.7. To say more, fix $C \in \mathcal{C}$ that is not of the form $\mathbb{R} \times \gamma$ with $\gamma$ an elliptic Reeb orbit. Suppose that $\eta \in V_{0 C}$. Write the components of $\phi_{0}(\eta)$ as $\left(\left(\phi_{0 C^{\prime}}\right)_{C^{\prime} \in \mathcal{C}},\left(\phi_{0 \gamma_{-}}\right)_{\gamma \in \Xi_{\Sigma+}},\left(\phi_{0_{\gamma+}}\right)_{\gamma \in \Xi_{\Sigma+}}\right)$. Set $\phi_{0 C^{\prime}}=0$ unless $C^{\prime}=C$. Meanwhile, set $\phi_{0 C}=\Sigma_{\mathcal{E} \in \mathcal{E}_{C}}\left(1-\chi_{\mathcal{E} *}^{\prime}\right) \eta$, where $\mathcal{E}_{C}$ again denotes the set of ends of $C$, and where $\chi_{\mathcal{E} *}^{\prime}$ is defined as follows: If $\gamma$ is an elliptic Reeb orbit in either $\Xi_{\Sigma_{-}}$or $\Xi_{\Sigma_{+}}$, then $\chi_{\mathcal{E} *}^{\prime}$ has support on $\mathcal{E}$ where it equals $\chi\left(\left(R_{\mathcal{E}}+3.5 R-|w|\right) / R\right)$. If $\gamma$ is a hyperbolic Reeb orbit, then $\chi_{\mathcal{E} *}^{\prime}$ has support on where it equals $\chi\left(\left(T^{\prime}+2.5 R-|w|\right) / R\right)$. Meanwhile, $\phi_{0 \gamma-}$ for $\gamma \in \Xi_{\Sigma_{-}}$and $\phi_{0 \gamma+}$ for $\gamma \in \Xi_{\Sigma_{+}}$are determined by $\phi_{0 C}$ using (5-12) in [14].

The map $\phi_{1}$ is defined in the same manner. 
Step 5 With the maps $\phi_{0}$ and $\phi_{1}$ so defined, it remains now to establish the assertions that are made by Lemma 3.8's third bullet. This is done with the help of the next lemma. This upcoming lemma reintroduces the $\mathcal{L}^{2}$-orthogonal projection $\mathbb{P}$ onto the $\mathcal{L}^{2}$-orthogonal complement of $\phi_{1}\left(V_{1}\right)$. The constant $R$ in this lemma is from Section 4.c in [14].

Lemma 3.10 Given $Q \geq 1$ and given the assumptions made by Lemma 3.8, there exists $\kappa>1$ such that if $r \geq \kappa^{2}$ and $R \geq \kappa$, then the following is true:

- Both $\phi_{0}$ and $\phi_{1}$ are nearly isometric in the sense that

(a) If $u \in V_{0}$, then $\left(1-\kappa R^{-1}\right)\|u\|_{L_{1}^{2}} \leq\left\|\phi_{0}(u)\right\|_{\mathcal{K}_{1}^{2}} \leq(1+\kappa R)\|u\|_{L_{1}^{2}}$.

(b) If $u \in V_{1}$, then $\left(1-\kappa R^{-1}\right)\|u\|_{L^{2}} \leq\left\|\phi_{0}(u)\right\|_{\mathcal{L}^{2}} \leq(1+\kappa R)\|u\|_{L^{2}}$.

- Let $\Delta_{*}^{\perp}$ denote the map from the $\mathcal{K}_{1}^{2}$-orthogonal complement of $\phi_{0}\left(V_{0}\right)$ to the $\mathcal{L}^{2}$-orthogonal complement of $\phi_{1}\left(V_{1}\right)$ that is by restricting $\mathbb{P} \Delta_{*}$.

(a) The operator $\Delta_{*}^{\perp}$ is an isomorphism that obeys $\left\|\Delta_{*}^{\perp} \theta\right\|_{\mathcal{L}^{2}} \geq \kappa^{-1}\|\theta\|_{\mathcal{K}_{1}^{2}}$.

(b) If $u \in V_{0}$, then $\left\|\mathbb{P} \Delta_{*} \phi_{0}(u)\right\|_{\mathcal{L}^{2}} \leq \kappa R^{-1}\|u\|_{L_{1}^{2}}$.

Proof of Lemma 3.10 The arguments for Lemma 3.10 are essentially identical to those used to prove Lemma 2.7. Here is the only substantive change: Use the bounds provided by Lemma 3.9 on the elements in $V_{0}$ in $V_{1}$ in lieu of those asserted by (2-84) and (2-85). One perhaps nonobvious, but essentially cosmetic change must also be made: Suppose that $\gamma$ is a hyperbolic Reeb orbit in either $\Xi_{\Sigma-}$ or $\Xi_{\Sigma+}$. Let $\mathcal{E}$ denote the end of $\Sigma$ that lies in $U_{\gamma-}$ or $U_{\gamma+}$ as the case may be. The function $\chi_{\mathcal{E}}$ should be replaced by the function $\chi_{\mathcal{E} *}$ which is defined so as to have support on $\mathcal{E}$, and on $\mathcal{E}$ it is set equal to $\chi\left(\left(T^{\prime}+2 R-|w|\right) / R\right)$.

Step 6 To see that the projection of $\phi_{1}\left(V_{1}\right)$ to the cokernel of $\Delta_{*}$ is surjective, fix $\eta \in \mathcal{L}^{2}$ with nontrivial projection to the cokernel of $\Delta_{*}$. According to what is said by Lemma 3.10, there exists $\theta \in \mathcal{K}_{1}^{2}$ that is $\mathcal{K}_{1}^{2}$-orthogonal to $\phi_{0}\left(V_{0}\right)$ and is such that

$$
\Delta_{*} \theta=\eta-\eta^{\prime}
$$

with $\eta^{\prime} \in \phi_{1}\left(V_{1}\right)$. So $\eta^{\prime} \in \phi_{1}\left(V_{1}\right)$ and $\eta$ project to the same element in $\operatorname{cokernel}\left(\Delta_{*}\right)$. Now consider the assertion made by Part (b) of the third bullet in Lemma 3.8. To this end, suppose that $\lambda \in \mathcal{K}_{1}^{2}$ is mapped by $\Delta_{*}$ to $\phi_{1}\left(V_{1}\right)$. Suppose in addition that $\lambda$ is $\mathcal{K}_{1}^{2}$-orthogonal to $\phi_{0}\left(V_{0}\right)$. Then $\lambda$ is in the domain of $\Delta_{*}^{\perp}$, and since $\Delta_{*}^{\perp} \lambda=0$, Lemma 3.8 requires that $\lambda=0$. This implies that the $\mathcal{K}_{1}^{2}$ orthogonal projection maps $\phi_{0}\left(V_{0}\right)$ onto the space $\left\{\theta \in \mathcal{K}_{1}^{2}: \Delta_{*} \theta \in \phi_{1}\left(V_{1}\right)\right\}$. To complete the proof of Part (b), suppose that $u \in V_{0}$.

According to Lemma 3.10, there exists a unique $\lambda$ in the $\mathcal{K}_{1}^{2}$-orthogonal complement of $\phi_{0}\left(V_{0}\right)$ such that $\mathbb{P} \Delta_{*} \lambda=\mathbb{P} \Delta_{*} \phi_{0}(u)$. This implies that $x=\phi_{0}(u)+\lambda$ is in the 
space $\left\{\theta \in \mathcal{K}_{1}^{2}: \Delta_{*} \theta \in \phi_{1}\left(V_{1}\right)\right\}$. Lemma 3.10 asserts that $\|\lambda\|_{\mathcal{K}_{1}^{2}} \leq c_{0} R^{-1}\left\|\phi_{0}(u)\right\|_{\mathcal{K}_{1}^{2}}$ Since $\phi_{0 *}(u)$ is no further from $u$ than $x$, it follows that $\left\|\phi_{0 *}(u)-\phi_{0}(u)\right\|_{\mathcal{K}_{1}^{2}} \leq$ $c_{0} R^{-1}\left\|\phi_{0}(u)\right\|_{\mathcal{K}_{1}^{2}}$ also.

To prove Part (c), suppose that $\lambda$ is $\mathcal{K}_{1}^{2}$ orthogonal to $\left\{\theta \in \mathcal{K}_{1}^{2}: \Delta_{*} \theta \in \phi_{1}\left(V_{1}\right)\right\}$, and write $\lambda=\phi_{0}(u)+\lambda^{\perp}$ with $\lambda^{\perp}$ being $\mathcal{K}_{1}^{2}$ orthogonal to $\phi_{0}\left(V_{0}\right)$. Part (b) of the lemma requires that $\left\|\phi_{0}(u)\right\|_{\mathcal{K}_{1}^{2}} \leq c_{0} R^{-1}\|\lambda\|_{\mathcal{K}_{1}^{2}}$ This understood, $\left\|\lambda^{\perp}\right\|_{\mathcal{K}_{1}^{2}} \geq \frac{1}{2}\|\lambda\|_{\mathcal{K}_{1}^{2}}$ when $R \geq c_{0}$.

Meanwhile, what is said by Lemma 3.10 finds that $\left\|\lambda^{\perp}\right\|_{\mathcal{K}_{1}^{2}} \leq c_{0}\left\|\Delta^{\perp} \lambda^{\perp}\right\|_{\mathcal{L}^{2}}$ and, what with the small size of $u$, this is less than $c_{0}\left(\|\mathbb{P} \Delta \lambda\|_{\mathcal{L}^{2}}+R^{-1}\|\lambda\|_{\mathcal{K}_{1}^{2}}\right)$. Putting all of these inequalities together gives what is claimed by Part (c) of the third bullet.

Part 11 To start, take $T>4 R$ and $T^{\prime} \gg T$. Suppose that $C \in \mathcal{C}$ is not of the form $\mathbb{R} \times \gamma$ with $\gamma$ an elliptic Reeb orbit. Part 4 describes a Fredholm operator $\mathcal{D}_{C ; T, T}$, and a 1-parameter family of first order, Fredholm differential operators from $L_{1}^{2}(C ; N)$ to $L^{2}(C ; N)$ that begins with $\mathcal{D}_{C}$ and ends with $\mathcal{D}_{C ; T, T},$. Each member of this family differs from $\mathcal{D}_{C}$ by an $\mathbb{R}$-linear, compactly supported endomorphism. Parametrize this family by $[0,1]$ and use $\mathcal{D}_{C *}$ to denote the $* \in[0,1]$ member of the family. Given that $r$ and $R$ are sufficiently large, then each $* \in[0,1]$ version of the set $\left\{\mathcal{D}_{C *}\right\}_{C \in \mathcal{C}}$ leads to a Fredholm operator, $\mathfrak{D}_{\Sigma *}$, mapping $L_{1}^{2}\left(\mathbb{R} \times M ; i T^{*} M \oplus \mathbb{S} \oplus i \mathbb{R}\right)$ to $L^{2}\left(\mathbb{R} \times M ; i T^{*} M \oplus \mathbb{S} \oplus i \mathbb{R}\right)$. This operator is described in Part 7. Meanwhile, Lemma 3.5 describes a canonical isomorphism

$$
\operatorname{det}\left(\mathfrak{D}_{\Sigma *}\right) \approx \operatorname{det}\left(\bigoplus_{C \in \mathcal{C}} \mathcal{D}_{C *}\right) .
$$

Here, as in Lemma 3.5, the notation is such that when $C=\mathbb{R} \times \gamma$ with associated integer $m>1$, then $\mathcal{D}_{C *}$ is independent of the chosen parameter in $[0,1]$; and refers not to $\mathcal{D}_{C}$, but to the latter's pullback to the $m$-fold covering cylinder. Use $\mathfrak{D}_{\Sigma ; T, T}$, to denote the end-member, $\mathfrak{D}_{\Sigma *=1}$, of this family. Given (3-81) and (3-65), there is a canonical isomorphism

$$
\operatorname{Det}\left(\mathfrak{D}_{\Sigma ; T, T^{\prime}}\right) \approx \Lambda^{\text {top }}\left(\bigoplus_{n-} \mathbb{R}\right) \otimes \operatorname{det}\left(\bigoplus_{C \in \mathcal{C}} \mathcal{D}_{C \diamond}\right) \otimes \Lambda^{\text {top }}\left(\bigoplus_{n+} \mathbb{R}\right) .
$$

An orientation for the line $\operatorname{det}\left(\mathfrak{D}_{\Sigma ; T, T^{\prime}}\right)$ determines an orientation for $\operatorname{det}\left(\mathfrak{D}_{\Sigma}\right)$ since the two operators in question are the end points of a path of Fredholm operators.

What is written on the right hand side of (3-82) is a tensor product of three lines. What follows here describes a decomposition of $\mathfrak{D}_{\Sigma ; T, T^{\prime}}$, in terms of three operators on $C^{\infty}\left(\mathbb{R} \times M ; i T^{*} M \oplus \mathbb{S} \oplus i \mathbb{R}\right)$, with each accounting for one of the lines on the right hand side of (3-82). These operators are denoted as $\mathfrak{D}_{-}, \mathfrak{D}_{\Sigma \diamond}$, and $\mathfrak{D}_{+}$.

What follows provides a quick definition of these operators. Given $x \in \mathbb{R}$, use $\lambda_{x}$ to denote the action on $C^{\infty}\left(\mathbb{R} \times M ; i T^{*} M \oplus \mathbb{S} \oplus i \mathbb{R}\right)$ of pullback via the map that 
sends $(s, \cdot)$ to $(s-x, \cdot)$. Thus, $\left.\left(\lambda_{x} \mathfrak{f}\right)\right|_{s}=\left.\mathfrak{f}\right|_{s-x}$. This understood, set

$$
\begin{aligned}
\mathfrak{D}_{-} & =\lim _{T^{\prime} \rightarrow \infty} \lambda_{T^{\prime}} \mathfrak{D}_{\Sigma ; T, T^{\prime}} \lambda_{-T^{\prime}}, \\
\mathfrak{D}_{\Sigma \diamond} & =\lim _{T^{\prime} \rightarrow \infty} \mathfrak{D}_{\Sigma ; T, T^{\prime}}, \\
\mathfrak{D}_{+} & =\lim _{T^{\prime} \rightarrow \infty} \lambda_{-T^{\prime}} \mathfrak{D}_{\Sigma ; T, T^{\prime}} \lambda_{T^{\prime}} .
\end{aligned}
$$

There is another way to define these operators. Start with $\mathfrak{D}_{-}$. Define $\Sigma_{-}$to be the

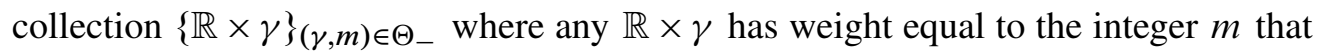
is associated to $\gamma$ as a member of $\Theta_{-}$. Let $\mathfrak{c}_{-}$denote the solution to (1-8) that is constructed from $\Theta_{-}$via $\Phi^{r}$. Thus, $\mathfrak{c}_{-}=\lim _{s \rightarrow-\infty} \mathfrak{d}_{\Sigma}$. Introduce the operator

$$
\mathfrak{D}_{\Sigma-}=\frac{\partial}{\partial s}+\mathfrak{L}_{\mathfrak{c}_{-}}
$$

where $\mathfrak{L}_{\mathfrak{c}}$ is the operator that is depicted in (1-9). To continue, let $\gamma$ denote a hyperbolic Reeb orbit in $\Xi_{\Sigma_{-}}$and let $C=\mathbb{R} \times \gamma$. Associated to $C$ is the operator

$$
\mathcal{D}_{C}=\frac{1}{2}\left(\frac{\partial}{\partial w}+i \frac{\partial}{\partial t}+\frac{1}{2} k\right) \eta+i \varepsilon e^{i k t} \bar{\eta},
$$

where $k$ is the rotation number of $\gamma$. Define $\mathcal{D}_{C *}$ to be the operator $\mathcal{D}_{0-}$ that is depicted in (3-56). Use the collection $\left\{\mathcal{D}_{C *}\right\}_{C \in \mathcal{C}}$ as instructed in Part 7 to define the operator $\mathfrak{D}_{\Sigma_{-*}}$. The latter is $\mathfrak{D}_{-}$.

There is an analogous definition of $\mathfrak{D}_{+}$, this using the data from $\Theta_{+}$and the operator $\mathcal{D}_{0+}$ that is depicted in (3-57). Meanwhile, the operator $\mathfrak{D}_{\Sigma \diamond}$ is obtained from $\mathfrak{D}_{\Sigma}$ using the constructions in Part 7 with any given $C \in \mathcal{C}$ version of $\mathcal{D}_{C *}$ set equal to the operator $\mathcal{D}_{C} \diamond$ that is described in Part 5.

Lemma 3.11 There exists $\kappa>1$ such that if $r \geq \kappa^{2}$ and $R \geq \kappa$, then the following is true: Each of the operators $\mathfrak{D}_{-}, \mathfrak{D}_{+}$and $\mathfrak{D}_{\Sigma \diamond}$ maps the space $L_{1}^{2}\left(\mathbb{R} \times M ; i T^{*} M \oplus S \oplus i \mathbb{R}\right)$ to $L^{2}\left(\mathbb{R} \times M ; i T^{*} M \oplus S \oplus i \mathbb{R}\right)$ as a Fredholm operator. In this regard, the operator $\mathfrak{D}_{-}$has index $-n_{-}$and trivial kernel; the operator $\mathfrak{D}_{+}$hast index $n_{+}$and trivial cokernel; and the operator $\mathfrak{D}_{\Sigma \diamond}$ has index $1-n_{-}+n_{+}$. Moreover, there are canonical isomorphisms

$$
\begin{aligned}
\operatorname{det}\left(\mathfrak{D}_{-}\right) & \approx \Lambda^{\mathrm{top}}\left(\bigoplus_{n_{-}} \mathbb{R}\right), \\
\operatorname{det}\left(\mathfrak{D}_{+}\right) & \approx \Lambda^{\operatorname{top}}\left(\bigoplus_{n_{+}} \mathbb{R}\right), \\
\operatorname{det}\left(\mathfrak{D}_{\Sigma 0}\right) & \approx \operatorname{det}\left(\bigoplus_{C \in \mathcal{C}} \mathcal{D}_{C \diamond}\right), \\
\operatorname{det}\left(\mathfrak{D}_{\Sigma ; T, T^{\prime}}\right) & \approx \operatorname{det}\left(\mathfrak{D}_{-}\right) \otimes \operatorname{det}\left(\mathfrak{D}_{\Sigma \diamond}\right) \otimes \operatorname{det}\left(\mathfrak{D}_{+}\right),
\end{aligned}
$$

with the following property: The isomorphism in (3-82) is identical to that obtained by composing this last isomorphism with the preceding three. 
Proof of Lemma 3.11 The assertions that $\mathfrak{D}_{-}, \mathfrak{D}_{+}$, and $\mathfrak{D}_{\Sigma} \diamond$ are Fredholm follow from Lemma 3.5. The assertion about the index, kernel and cokernel of $\mathfrak{D}_{-}, \mathfrak{D}_{+}$, follow from Lemma 3.6. This lemma supplies the canonical isomorphism between $\bigoplus_{n-} \mathbb{R}$ and the kernel of $\mathfrak{D}_{-}$. Here, each copy of $\mathbb{R}$ corresponds to the kernel of a copy of (3-58)'s operator $\mathcal{D}_{-}$; one such copy for each hyperbolic Reeb orbit in $\Xi_{\Sigma_{-}}$ with even rotation number. Likewise, Lemma 3.6 supplies the canonical isomorphism between its cokernel and $\bigoplus_{n_{+}} \mathbb{R}$. In this case, each copy of $\mathbb{R}$ is associated to a hyperbolic Reeb orbit in $\Xi_{\Sigma+}$ with trivial rotation number. The assertion about the index of $\mathfrak{D}_{\Sigma \diamond}$ can be proved by using Lemma 3.4 given that the $T^{\prime}>8 R_{*}$ version of the operator $\mathfrak{D}_{\Sigma, T^{\prime}, T}$ is very nearly $s$-invariant where $|s| \in\left[\frac{3}{4} T^{\prime}, T^{\prime}\right]$.

The assertion about $\operatorname{det}\left(\mathfrak{D}_{\Sigma ; T, T^{\prime}}\right)$ also follows by taking $T^{\prime}$ large and invoking Lemma 3.4. The compatibility of the isomorphisms in Lemma 3.11 and that in (3-82) is a consequence of two facts: First, both isomorphisms invoke Lemma 3.4. Second, the isomorphisms that appear in Lemma 3.4 are defined using cut-off functions that have no substantive affect on the various constructions that are used in Parts 9 and 10. The details of justification are straightforward and omitted.

Part 12 This part focuses on the operator $\mathfrak{D}_{\Sigma \diamond}$. To start, suppose that $C \in \mathcal{C}$ is not of the form $\mathbb{R} \times \gamma$ where $\gamma$ is an elliptic Reeb orbit. As the corresponding operator $\mathcal{D}_{C} \diamond$ is $\mathbb{C}$-linear at large $|s|$ on $C$, it follows that there is a deformation of $\mathcal{D}_{C} \diamond$ through Fredholm operators to a $\mathbb{C}$-linear operator. In particular, choose such a deformation, parametrized by $[0,1]$, whose $\sigma \in[0,1]$ element is defined by replacing $\mu_{C} \diamond$ in (3-63) with $(1-\sigma) \mu_{C} \diamond$. Let $\mathcal{D}_{C \diamond \sigma}$ denote the resulting $\sigma \in[0,1]$ operator.

The constructions in Part 7 can now be applied using each $\sigma \in[0,1]$ version of the $\left\{\mathcal{D}_{C *}=\mathcal{D}_{C \diamond \sigma}\right\}_{C \in \mathcal{C}}$ to define a 1-parameter family $\left\{\mathfrak{D}_{\Sigma *}=\mathfrak{D}_{\Sigma \diamond \sigma}\right\}_{\sigma \in[0,1]}$ of Fredholm operators mapping $L_{1}^{2}\left(\mathbb{R} \times M ; i T^{*} M \oplus \mathbb{S} \oplus i \mathbb{R}\right)$ to $L^{2}\left(\mathbb{R} \times M ; i T^{*} M \oplus \mathbb{S} \oplus i \mathbb{R}\right)$. There is of course, a canonical isomorphism between $\operatorname{det}\left(\mathfrak{D}_{\Sigma \diamond}\right)$ and any $\sigma \in[0,1]$ version $\operatorname{det}\left(\mathfrak{D}_{\Sigma \triangleright \sigma}\right)$. Meanwhile, Lemma 3.5 provides the canonical isomorphisms

$$
\operatorname{det}\left(\mathfrak{D}_{\Sigma \triangleright \sigma}\right) \approx \operatorname{det}\left(\bigoplus_{C \in \mathcal{C}} \mathcal{D}_{C \diamond \sigma}\right)
$$

As noted at the end of Part 5, the line $\operatorname{det}\left(\bigoplus_{C \in \mathcal{C}} \mathcal{D}_{C} \diamond_{1}\right)$ has its positive orientation because each $\mathcal{D}_{C \diamond 1}$ is complex. This orientation induces a canonical orientation on $\operatorname{det}\left(\mathfrak{D}_{\Sigma \diamond 1}\right)$.

Now suppose $\Sigma^{\prime}$ is a second element in $\mathcal{M}_{1}\left(\Theta_{-}, \Theta_{+}\right)$. There is a corresponding operator $\mathfrak{D}_{\Sigma^{\prime} \diamond 1}$ and the two operators $\mathfrak{D}_{\Sigma \diamond 1}$ and $\mathfrak{D}_{\Sigma^{\prime} \diamond 1}$ have the same $s \rightarrow-\infty$ limit and they have the same $s \rightarrow \infty$ limit. Since they have the same index, there is a 1-parameter family of first order, Fredholm operators that maps $L_{1}^{2}\left(\mathbb{R} \times M ; i T^{*} M \oplus \mathbb{S} \oplus i \mathbb{R}\right)$ to 
$L^{2}\left(\mathbb{R} \times M ; i T^{*} M \oplus \mathbb{S} \oplus i \mathbb{R}\right)$ with one end member $\mathfrak{D}_{\Sigma \diamond 1}$ and the other $\mathfrak{D}_{\Sigma^{\prime} \diamond 1}$. Such a homotopy can be used to compare the canonical orientations of $\operatorname{det}\left(\mathfrak{D}_{\Sigma \diamond 1}\right)$ with that for $\operatorname{det}\left(\mathfrak{D}_{\Sigma^{\prime} \diamond 1}\right)$.

Lemma 3.12 Let $\Sigma$ and $\Sigma^{\prime}$ denote two elements in $\mathcal{M}_{1}\left(\Theta_{-}, \Theta_{+}\right)$. Then the canonical orientations of $\operatorname{det}\left(\mathfrak{D}_{\Sigma \diamond 1}\right)$ and $\operatorname{det}\left(\mathfrak{D}_{\Sigma^{\prime} \diamond 1}\right)$ agree.

This lemma is proved in the upcoming Part 13.

Assume for the moment that Lemma 3.12 is true. Let $\Sigma$ and $\Sigma^{\prime}$ denote two elements in $\mathcal{M}_{1}\left(\Theta_{-}, \Theta_{+}\right)$. Their respective components are oriented by respective orientations for the $\Sigma$ and $\Sigma^{\prime}$ versions of (3-52). The latter are oriented by the respective versions of (3-65). In this regard, both versions of the line $\operatorname{det}\left(\bigoplus_{C \in \mathcal{C}} \mathcal{D}_{C \diamond}\right)$ are oriented by the canonical orientations for the respective $\Sigma$ and $\Sigma^{\prime}$ versions of $\operatorname{det}\left(\bigoplus_{C \in \mathcal{C}} \mathcal{D}_{C \diamond 1}\right)$. As noted at the end of Part 6, the respective orientations of the $\Sigma$ and $\Sigma^{\prime}$ components of $\mathcal{M}_{1}\left(\Theta_{-}, \Theta_{+}\right)$define the same section, $o_{\text {ech }}$, of the orientation sheaf $\Lambda\left(\Theta_{-}, \Theta_{+}\right)$, this coming from a system of coherent orientations. These orientations also serve to orient the components of the instantons $\mathfrak{d}_{\Sigma}$ and $\mathfrak{d}_{\Sigma}$, via the $\mathfrak{D}_{\Sigma *}=\mathfrak{D}_{\Sigma}$ versions of Lemma 3.5. It follows from Lemmas 3.12 and 3.11 that these orientations of the instanton moduli spaces define one and the same section of the orientation sheaf $\Lambda\left(\mathfrak{c}_{-}, \mathfrak{c}_{+}\right)$. The latter is denoted by $\mathfrak{o}\left(\mathfrak{c}_{-}, \mathfrak{c}_{+}\right)$.

Part 13 This part of the proof contains:

Proof of Lemma 3.12 The vector bundle $T^{*} M \oplus \mathbb{R}$ can be viewed as a rank 2, complex vector bundle as follows: First, view $T^{*} M \oplus \mathbb{R}$ as $T^{*}(\mathbb{R} \times M)$ by identifying the $\mathbb{R}$ factor in the former with the span of $d s$ in the latter. Then, use the almost complex structure $J$ to view $T^{*}(\mathbb{R} \times M)$ as a complex vector bundle. As $\mathbb{S}$ is given as a complex vector bundle, so the bundle $i T^{*} M \oplus \mathbb{S} \oplus i \mathbb{R}$ can be viewed as a complex vector bundle. The principle symbol of any $\mathfrak{D}_{(\cdot)}$ is $\mathbb{C}$-linear with respect to this complex vector bundle structure. Moreover, the operator $\mathfrak{D}_{\Sigma \diamond 1}$ is very nearly $\mathbb{C}$-linear. To make this precise, introduce the operator $\mathfrak{D}_{\Sigma \diamond \diamond}$ that is defined so as to take a section $\mathfrak{f}$ of $i T^{*} M \oplus \mathbb{S} \oplus i \mathbb{R}$ to

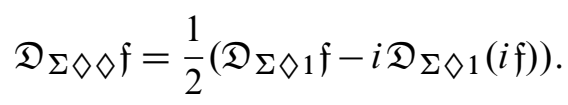

Here, $i=\sqrt{-1}$. By construction, the operator $\mathfrak{D}_{\Sigma 00}$ is $\mathbb{C}$-linear and it differs from $\mathfrak{D}_{\Sigma 01}$ by a zero-th order endomorphism.

Lemma 3.13 There exists $\kappa>1$ such that when $r \geq \kappa^{2}$ and $R \geq \kappa$, the operator $\mathfrak{D}_{\Sigma \diamond \diamond}$ is Fredholm from $L_{1}^{2}\left(\mathbb{R} \times M ; i T^{*} M \oplus \mathbb{S} \oplus i \mathbb{R}\right)$ to $L^{2}\left(\mathbb{R} \times M ; i T^{*} M \oplus \mathbb{S} \oplus i \mathbb{R}\right)$. Moreover, it is homotopic through first order, Fredholm differential operators to $\mathfrak{D}_{\Sigma \diamond 1}$. 
This lemma is proved momentarily.

To continue, note that as $\mathfrak{D}_{\Sigma \diamond \diamond}$ is a $\mathbb{C}$-linear operator, the line $\operatorname{det}\left(\mathfrak{D}_{\Sigma \diamond \diamond}\right)$ has a canonical positive orientation. The latter induces via Lemma 3.13's homotopy an orientation for the line $\operatorname{det}\left(\mathfrak{D}_{\Sigma} \diamond 1\right)$.

Lemma 3.14 There exists $\kappa>1$ such that when $r \geq \kappa^{2}$ and $R \geq \kappa$, then the orientation on $\operatorname{det}\left(\mathfrak{D}_{\Sigma \diamond 1}\right)$ that is induced from the positive orientation of $\operatorname{det}\left(\mathfrak{D}_{\Sigma \diamond \diamond}\right)$ is the same as that induced from the positive orientation on $\operatorname{det}\left(\bigoplus_{C \in \mathcal{C}} \mathcal{D}_{C \diamond 1}\right)$ via Lemma 3.5.

This lemma is also proved momentarily.

To complete the proof of Lemma 3.12, introduce the $\Sigma^{\prime}$ version of (3-87), this denoted by $\mathfrak{D}_{\Sigma^{\prime}} \diamond \diamond$. The latter is also $\mathbb{C}$-linear. Of course, the conclusions of Lemmas 3.13 and 3.14 apply to $\mathfrak{D}_{\Sigma^{\prime} \diamond \diamond}$ and $\mathfrak{D}_{\Sigma^{\prime} \diamond 1}$ also. Meanwhile, $\mathfrak{D}_{\Sigma \diamond \diamond}$ and $\mathfrak{D}_{\Sigma^{\prime} \diamond \diamond}$ are $\mathbb{C}$-linear Fredholm operators with the same domain and range Hilbert spaces, with the same index and with the same principle symbols. In addition, their respective $s \rightarrow-\infty$ limits agree as do their respective $s \rightarrow \infty$ limits. As a consequence, they are homotopic through such $\mathbb{C}$-linear, first order, Fredholm operators. This being the case, it follows from Lemma 3.14 that the respective positive orientations of $\operatorname{det}\left(\mathfrak{D}_{\Sigma \diamond \diamond}\right)$ and $\operatorname{det}\left(\mathfrak{D}_{\Sigma^{\prime} \diamond \diamond}\right)$ are identified by any such homotopy. This fact with Lemma 3.14 implies Lemma 3.12.

Proofs of Lemmas 3.13 and 3.14 Write $\mathfrak{D}_{\Sigma \diamond 1}=\mathfrak{D}_{\xi \diamond 1}+r^{1 / 2} \mathfrak{e}_{q} *(\cdot)$. The operator $\mathfrak{D}_{\xi \diamond 1}$ has its version of (3-67), (3-68) and Lemma 3.8 with $\mathfrak{D}_{\xi *}=\mathfrak{D}_{\xi \diamond 1}$, with $p_{*}=p_{\diamond 1}$ and $p_{*}^{\prime}=p_{\diamond 1}^{\prime}$, and with $\Delta_{*}=\Delta_{\diamond 1}$. It is important to note with regard to Lemma 3.8 that each $C \in \mathcal{C}$ version of $V_{0 C}$ and $V_{1 C}$ can be taken to be a complex vector space. As noted, this is because each version of $\mathcal{D}_{C \diamond 1}$ is $\mathbb{C}$-linear. By the way, no generality lost by assuming that at most one of $V_{0 C}$ and $V_{1 C}$ is nontrivial for any given $C$. As done in Lemma 3.8, set $V_{0}=\bigoplus_{C \in \mathcal{C}} V_{0 C}$ and set $V_{1}=\bigoplus_{C \in \mathcal{C}} V_{1 C}$.

Now fix $\sigma \in[0,1]$ so as to consider

$$
\widehat{\mathfrak{D}}=\left(1-\frac{1}{2} \sigma\right) \mathfrak{D}_{\xi \diamond 1}-\frac{1}{2} \sigma i \mathfrak{D}_{\xi \diamond 1}(i \cdot)=\mathfrak{D}_{\xi \diamond 1}+\sigma\left(\mathfrak{D}_{\xi \diamond \diamond}-\mathfrak{D}_{\xi \diamond 1}\right) .
$$

Thus, the $\sigma=0$ version is $\mathfrak{D}_{\xi \diamond 1}$ and the $\sigma=1$ version is $\mathfrak{D}_{\xi \diamond \diamond}$. The (3-67) analog for $\mathfrak{D}$ is written schematically as

$$
\begin{aligned}
\left(1-\Pi_{\xi}\right) \mathfrak{D} \mathfrak{f} & =\left(\mathfrak{D}_{\xi \diamond 1}\right)^{\perp} \mathfrak{f}^{\perp}+p^{*}(\theta)+\mathfrak{r} \mathfrak{f}^{\perp}, \\
\mathfrak{t}^{\dagger}\left(\mathfrak{D}_{\xi} \mathfrak{f}\right) & =p^{* \prime}\left(\mathfrak{f}^{\perp}\right)+\Delta_{\diamond 1} \theta+\mathfrak{r}^{\prime} \theta .
\end{aligned}
$$


By virtue of (3-68), the terms $p^{*}$ and $p^{* \prime}$ obey

$$
\left\|p^{*}(\theta)\right\|_{2} \leq c_{0} r^{3 \sigma}\|\theta\|_{\|_{1}^{2}} \quad \text { and } \quad\left\|p^{* \prime}\left(\mathfrak{f}^{\perp}\right)\right\|_{\mathcal{L}^{2}} \leq c_{0} r^{-1 / 2+3 \sigma}\left\|\mathfrak{f}^{\perp}\right\|_{\mathbb{H}}
$$

Meanwhile, what are written as $\mathfrak{r}$ and $\mathfrak{r}^{\prime}$ obey

$$
\left\|\mathfrak{r} \mathfrak{f}^{\perp}\right\|_{2} \leq c_{0}\left\|\mathfrak{f}^{\perp}\right\|_{2} \quad \text { and } \quad\left\|\mathfrak{r}^{\prime} \theta\right\|_{2} \leq c_{0} r^{-1 / 2+8 \sigma}\|\theta\|_{2}
$$

With regard to these last bounds, the $\mathfrak{r}$ bound follows from the fact that $\mathfrak{D}_{\Sigma \diamond \diamond}-\mathfrak{D}_{\Sigma} \diamond 1$ is a zero-th order operator. The $\mathfrak{r}^{\prime}$ bound is a consequence of the fact that $\Delta_{01}$ is nearly $\mathbb{C}$-linear; this because each $C \in \mathcal{C}$ and $\gamma \in \Xi_{\Sigma_{ \pm}}$version of (2-78) for $\mathfrak{D}_{\Sigma \diamond 1}$ has $\mu_{*}=0$.

It follows from the bound on $\mathfrak{r}$ and the first bullet in (3-68) that $\mathfrak{D} \frac{\perp}{\Sigma}{ }^{\prime}+\mathfrak{r}$ is invertible as a map from $\mathbb{H}_{\xi}^{\perp}$ to $\mathbb{L}_{\xi}^{\perp}$ with inverse whose norm is bounded by $c_{0}$. As a consequence, Step 1 of the proof of Lemma 3.5 can be repeated with only notational changes to prove the following two assertions: The kernel of $\hat{\mathfrak{D}}$ is canonically isomorphic to the kernel of $\Delta_{\diamond 1}+e^{*}+\mathfrak{r}^{\prime}$; and the cokernel of $\hat{\mathfrak{D}}$ is canonically isomorphic to the cokernel of $\Delta_{\diamond 1}+e^{*}+\mathfrak{r}^{\prime}$. Here, $e^{*}$ obeys the same bound as does $e_{*}=e \diamond 1$ in (3-70). As a consequence, there is a canonical isomorphism

$$
\operatorname{det}(\mathfrak{D}) \approx \operatorname{det}\left(\Delta_{\diamond 1}+e^{*}+\mathfrak{r}^{\prime}\right)
$$

Given the small norm for $e^{*}$ and for $\mathfrak{r}^{\prime}$ when $\mathfrak{r}$ is large, Step 2 in the proof of Lemma 3.5 can be repeated with only notational changes to see that $\hat{\mathfrak{D}}$ is Fredholm when $r$ and $R$ are large. This gives Lemma 3.13. By the same token, Step 3 in the proof of Lemma 3.5 can be repeated to obtain a canonical isomorphism between $\operatorname{det}\left(\Delta_{\diamond 1}+e^{*}+\mathfrak{r}^{\prime}\right)$ and $\left(\Lambda^{\text {top }} V_{0}\right) \otimes\left(\Lambda^{\text {top }} V_{1}\right)^{*}$. This with (3-92) implies what is asserted by Lemma 3.14.

Part 14 This part completes the proof of the second bullet in Theorem 1.2. There are two claims to address. The first is the claim that $\mathfrak{o}\left(\mathfrak{c}_{-}, \mathfrak{c}_{+}\right)$gives the same orientation on the $\Psi^{r}$ image as does the pushforward of $o_{\text {ech }}$. This follows directly from Lemma 3.6.

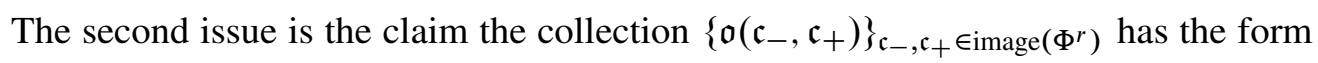

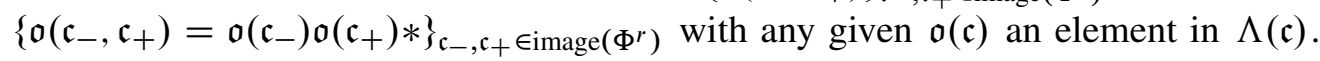
This claim is verified in the three steps that follow.

Step 1 The $\mathbb{Z} / 2 \mathbb{Z}$ module $\Lambda(\mathfrak{c})$ has the form $\Lambda\left(\mathfrak{c}, \mathfrak{c}_{0}\right)$ where $\mathfrak{c}_{0}$ can be any fixed, element in $\operatorname{Conn}(E) \times C^{\infty}(M ; \mathbb{S})$ whose version of (1-9) has trivial kernel. Choose $\mathfrak{c}_{0}=\left(A_{0}, \psi_{0}\right)$ with $\psi_{0}=\left(\alpha_{0}, \beta_{0}=0\right)$. Write $T^{*} M \oplus \mathbb{R}$ as $T^{*}(\mathbb{R} \times M)$ and use $J$ to view the latter as the underlying real bundle of a complex $\mathbb{C}^{2}$-bundle, $\mathbb{V} \rightarrow M$. 
This done, view $\mathfrak{L}_{\mathfrak{c}_{0}}$ as symmetric operator, $\mathbb{R}$-linear operator on $C^{\infty}(M ; \mathbb{V} \oplus \mathbb{S})$. Mimic what is done in (3-87) to define the $\mathbb{C}$-linear operator

$$
L_{0 \diamond}=\frac{1}{2}\left(\mathfrak{L}_{\mathfrak{c}_{0}}-i \mathfrak{L}_{\mathfrak{c}_{0}}(i(\cdot))\right) .
$$

This operator is symmetric and $\mathbb{C}$-linear. Moreover, it differs from $\mathfrak{L}_{0}$ by a zero-th order endomorphism of $\mathbb{V}$. If $\left(A_{0}, \alpha_{0}\right)$ is suitably generic, then $\mathfrak{L}_{0}$ has trivial kernel. Assume that such is the case.

Now introduce $\mathfrak{D}_{\mathfrak{c}_{0}}=\partial / \partial s+\mathfrak{L}_{\mathfrak{c}_{0}}$ and $\mathfrak{D}_{0 \diamond}=\partial / \partial s+\mathfrak{L}_{0 \diamond}$. View both as operators that map $L_{1}^{2}(\mathbb{R} \times M ; \mathbb{V} \oplus \mathbb{S})$ to $L^{2}(\mathbb{R} \times M ; \mathbb{V} \oplus \mathbb{S})$. Both are Fredholm operators. Define

$$
\mathfrak{D}_{0 *}=(1-\chi) \mathfrak{D}_{0}+\chi \mathfrak{D}_{0 \diamond}
$$

This operator is identical to $\mathfrak{D}_{\mathfrak{c}_{0}}$ where $s \gg 1$ and it is identical to $\mathfrak{D}_{0 \diamond}$ where $s \ll-1$. The operator $\mathfrak{D}_{0 *}$ is also a Fredholm operator. This understood, fix once and for all an orientation for its determinant line.

For $T \geq 0$, define $\mathfrak{D}_{0 * T}$ by replacing $\chi$ in (3-95) by $\chi((\cdot)-T)$.

Step 2 Let $\mathfrak{c} \in \operatorname{Conn}(E) \times C^{\infty}(M ; \mathbb{S})$ denote a solution to (1-8) that gives an element in the image of $\Phi^{r}$. Choose a smooth map $\mathfrak{d}: \mathbb{R} \rightarrow \operatorname{Conn}(E) \times C^{\infty}(M ; \mathbb{S})$ that is equal to $\mathfrak{c}$ for $s \leq-1$ and equal to $\mathfrak{c}_{0}$ for $s \geq 1$. Let $\mathfrak{D}_{\mathfrak{d}}$ denote the corresponding version of $(1-12)$. An element in $\Lambda\left(\mathfrak{c}, \mathfrak{c}_{0}\right)$ is specified by an orientation of $\operatorname{det}\left(\mathfrak{D}_{\mathfrak{d}}\right)$.

To obtain such an orientation, fix $T \gg 1$ and define the operator $\mathfrak{D}_{\mathfrak{d}, T}$ as follows: For $-1 \leq s \leq 1$, this operator is $\mathfrak{D}_{\mathfrak{d}}$. For $s>1$ where $\mathfrak{D}_{\mathfrak{d}}=\mathfrak{D}_{\mathfrak{c}_{0}}$, write

$$
\mathfrak{D}_{\mathfrak{d}, T}=\chi_{2} \mathfrak{D}_{\mathfrak{c}_{0}}+\left(1-\chi_{2}\right) \mathfrak{D}_{0 * T}
$$

and where $s<-1$ where $\mathfrak{D}_{\mathfrak{d}}$ agrees with $\mathfrak{D}_{\mathfrak{c}}=\partial / \partial s+\mathfrak{L}_{\mathfrak{c}}$, set

$$
\mathfrak{D}_{\mathfrak{d}, T}=\left(1-\chi_{-2}\right) \mathfrak{D}_{\mathfrak{c}}+\chi_{-2}\left(\left(1-\chi_{-T}\right) \mathfrak{D}_{-}+\chi_{-T} \mathfrak{D}_{\mathfrak{c}}\right)
$$

where $\mathfrak{D}$ is defined as in Part 11 using $\mathfrak{c}$ for what is denoted there by $\mathfrak{c}_{-}$. Note that the latter has the form as depicted in (3-66).

Denote by $\mathfrak{D}_{\mathfrak{d} \diamond}$ the operator that is defined as follows: For $-1 \leq s \leq 1$, it is the same as $\mathfrak{D}_{\mathfrak{d}} \diamond$. For $s<-1$, it is

$$
\left(1-\chi_{-2}\right) \mathfrak{D}_{\mathfrak{c}}+\chi_{-2}\left(\left(1-\chi_{-T}\right) \mathfrak{D}_{-}+\chi_{-T} \mathfrak{D}_{\mathfrak{c}}\right) .
$$

For $s>1$, the operator $\mathfrak{D}_{\mathfrak{d} \diamond}$ is

$$
\mathfrak{D}_{\mathfrak{d} \diamond}=\chi_{-2} \mathfrak{D}_{\mathfrak{c}_{0}}+\left(1-\chi_{-2}\right) \mathfrak{D}_{0 \diamond}
$$

The operator $\mathfrak{D}_{\mathfrak{d} \diamond}$ is obtained by taking $T \rightarrow \infty$ in the definition of $\mathfrak{D}_{\mathfrak{d}, T}$. 
Step 3 It follows from Lemma 3.4 that there is a canonical isomorphism

$$
\operatorname{det}\left(\mathfrak{D}_{\delta}\right)=\operatorname{det}\left((1-\chi) \mathfrak{D}_{-}+\chi \mathfrak{D}_{\mathfrak{c}}\right) \otimes \operatorname{det}\left(\mathfrak{D}_{\mathfrak{d} \diamond}\right) \otimes \operatorname{det}\left(\mathfrak{D}_{0 *}\right) .
$$

An orientation for $\operatorname{det}\left(\mathfrak{D}_{0 *}\right)$ has already been selected. As will now be explained, the line $\operatorname{det}\left(\mathfrak{D}_{\mathfrak{d} \diamond}\right)$ has a canonical orientation. Indeed, such is the case because it is homotopic through a path of Fredholm operators to one that is $\mathbb{C}$-linear. A homotopy of this sort exists because $\mathfrak{D}_{\mathfrak{d}} \diamond$ was constructed so as to be $\mathbb{C}$-linear where $|s| \gg 1$. Granted what was just said, it follows that $\operatorname{det}\left(\mathfrak{D}_{\mathfrak{d}}\right)$ is oriented by a choosing an orientation for the determinant line bundle of $(1-\chi) \mathfrak{D}_{-}+\chi \mathfrak{D}_{\mathfrak{c}}$. It follows from Lemmas 3.5 and 3.6 that an orientation for the latter is obtained from an orientation of $\operatorname{det}\left(\mathfrak{D}_{-}\right)$.

Write $\mathfrak{c}$ 's image in $\mathcal{M}^{r}$ as $\Phi^{r}(\Theta)$. Note that the first bullet of Lemma 3.11 has the following consequence: The line $\operatorname{det}\left(\mathfrak{D}_{-}\right)$is oriented by the following data: First, an orientation for the kernel of the various versions of the operator $\mathfrak{D}_{0-}$ in (3-56) that are associated to the hyperbolic Reeb orbits with even rotation number that appear in $\Theta$.

Second, an ordering of this same set of Reeb orbits.

Given what just been said, the claim

$$
\left\{\mathfrak{o}\left(\mathfrak{c}_{-}, \mathfrak{c}_{+}\right)\right\}_{\mathfrak{c}_{-}, \mathfrak{c}_{+} \in \operatorname{image}\left(\Phi^{r}\right)}=\left\{\mathfrak{o}\left(\mathfrak{c}_{-}\right) \mathfrak{o}\left(\mathfrak{c}_{+}\right)^{*}\right\}_{\mathfrak{c}_{-}, \mathfrak{c}_{+} \in \operatorname{image}\left(\Phi^{r}\right)}
$$

follows jointly from (3-99) and the final two assertions of Lemma 3.11.

\section{Index to the notation}

$a$ : the contact $1-$ form

$K^{-1}:$ the kernel of $a$, oriented by $-d a$

$c_{1}(K)$ : the first Chern class of $K$

$v$ : the Reeb vector field

Reeb orbit: a closed integral curve of $v$, typically denoted by $\gamma$

$\Gamma$ : a class in $H_{1}(M ; \mathbb{Z})$

$\Theta$ : a finite set of pairs $(\gamma, m)$ with $\gamma$ a Reeb orbit and $m$ a positive integer

$\mathcal{Z}$ : a set of $\Theta$ 's as above satisfying particular Part 1 of Section 1.a constraints

$\ell_{\gamma}$ : when $\gamma$ is a Reeb orbit, the integral of $a$ Part 1 of Section 1.a along $\gamma$

Part 1 of Section 1.a

Part 1 of Section 1.a

Part 1 of Section 1.a

Part 1 of Section 1.a

Part 1 of Section 1.a

Part 1 of Section 1.a

Part 1 of Section 1.a 
$\mathcal{Z}^{L}$ : a subset of $Z$ with a length bound given Part 1 of Section 1.a by $L$ on the Reeb orbits.

$(\nu, \mu)$ defined for a given Reeb orbit

$\mathcal{L}$ : a differential operator associated to a Reeb orbit

elliptic, hyperbolic

rotation number $\mathrm{R}$ : the rotation number for an elliptic Reeb orbit

Conley-Zehnder index

$I\left(\Theta_{-}, \Theta_{+}, Z\right)$

$\operatorname{deg}_{\text {ech }}(\Theta)$

$\mathfrak{C}_{m}$ : the vortex moduli space

the vortex equations

h

$\mathfrak{C}_{(\gamma, m)}$

nondegenerate map from $S^{1}$ to $\mathfrak{C}_{m}$

$\mathfrak{C} \Theta, \mathfrak{C} \Theta *$

symmetric vortex

$\mathcal{R}$

$\operatorname{deg}_{\mathfrak{C}}(\mathfrak{c})$

the Riemannian metric

$\mathbb{S}$ : the bundle of spinors for a Spin ${ }^{\mathbb{C}}$-structure

Conn $(E)$ : the Hermitian connections on $E$

$\mu$ : a chosen $1-$ form with bounded derivatives of all orders

$A_{K}$ : a chosen Hermitian connection on $K^{-1}$

$B_{A}$ : the metric Hodge dual of the curvature of a connection $A$

$\psi:$ a section of $\mathbb{S}$

$D_{A}$ : the Dirac operator

$\psi^{\dagger} \tau \psi$

$\mathrm{cl}(\cdot)$ : the Clifford multiplication map

$\mathcal{M}^{r}$ : the space of equivalence classes of solutions to (1-8)

$\mathfrak{L}_{c}$

nondegenerate solution to (1-8)

$\operatorname{deg}_{\mathrm{SW}}(\mathfrak{c})$

$\mathfrak{C} \mathcal{Z}^{*}$
Equation (1-1)

Equation (1-2)

Step 2 of Part 2 in Section 1.a

Step 2 of Part 2 in Section 1.a

Step 3 of Part 2 in Section 1.a Equation (1-4)

Step 6 of Part 2 in Section 1.a

Step 1 of Part 3 in Section 1.a

Equation (1-5)

Equation (1-6)

Step 1 of Part 3 in Section 1.a

Step 2 of Part 3 in Section 1.a

Step 2 of Part 3 of Section 1.a

Step 3 of Part 3 in Section 1.a

Step 3 of Part 3 of Section 1.a

Step 3 of Part 3 of Section 1.a

Part 4 of Section 1.a

Part 4 of Section 1.a

Part 4 of Section 1.a

Part 4 of Section 1.a

Part 4 of Section 1.a

Part 4 of Section 1.a

Part 4 of Section 1.a

Part 4 of Section 1.a

Part 4 of Section 1.a

Part 4 of Section 1.a

Part 4 of Section 1.a

Equation (1-9)

Part 4 of Section 1.a

Part 4 of Section 1.a

the paragraph prior to Theorem

1.1 in Section 1.a 
$\mathcal{J}_{a}$ : a certain set of almost complex structures $\mathcal{Z}_{\text {ech }}:$ a particular subset of $\mathcal{Z}$ $\mathcal{Z}_{\text {ech }}^{L}$ : a particular subset of $\mathcal{Z}_{\text {ech }}$ $\mathcal{M}_{1}\left(\Theta_{-}, \Theta_{+}\right)$

$\mathfrak{o}_{\text {ech }}$

$\mathcal{D}_{C}$

$\left(v_{C}, \mu_{C}\right)$

instanton

$\mathfrak{D}_{\mathfrak{d}}$

$\mathbb{H}$ in Section 2

$\mathbb{H}$ in Section 3

$\|\cdot\|_{\mathbb{H}}$ in Section 2

$\|\cdot\|_{\mathbb{H}}$ in Section 3

nondegenerate instanton

$\mathcal{M}_{1}\left(\mathfrak{c}_{-}, \mathfrak{c}_{+}\right)$

$\mathfrak{P}\left(\mathfrak{c}_{-}, \mathfrak{c}_{+}\right)$

orientation sheaf in the Seiberg-Witten context orientation sheaf in the embedded contact homology context

$\Lambda\left(\mathfrak{c}_{-}, \mathfrak{c}_{+}\right)$

$\Lambda(\mathfrak{c})$

$\mathcal{M}^{r^{*}}$

coherent orientations in the Seiberg-Witten context

coherent system of orientations in the embedded contact homology context

$\chi$ : a cut-off function chosen for eternity

$c_{0}$ : a constant that is greater than 1 and is independent of what ever relevant data is under consideration. The precise value of $c_{0}$ can increase between subsequent appearances
$\left(A_{\mathfrak{J}}, \psi_{\mathfrak{J}}\right)$
$\left(b_{\mathfrak{J}}, \eta_{\mathfrak{J}}, \phi_{\mathfrak{J}}\right)$
$\zeta_{\gamma}$
$\mathfrak{b}(\zeta)$
$\mathfrak{c}_{\Theta_{\mathfrak{J}}},\left(A_{\mathfrak{J}}, \psi_{\mathfrak{J}}\right)$

Part 1 of Section 1.b

Part 1 of Section 1.b

Part 1 of Section 1.b

Part 1 of Section 1.b

Part 2 of Section 1.b

Equation (1-10)

Part 2 of Section 1.b

Part 3 of Section 1.b

Equation (1-12)

Step 4 of Section 2.a, Step 1 of

Section 3.b in [14]

Part 2 of Section 3.a, Part 1 of

Section 6.a in [14]

Step 4 of Section 2.a, (3-7)

in [14]

Equation (1-13), (6-2) in [14]

Part 3 of Section 1.b

Part 4 of Section 1.b

Part 5 of Section 1.b

Part 5 of Section 1.b

Section 3.b

Part 5 of Section 1.b

Part 5 of Section 1.b

Part 6 of Section 1.b

Part 6 of Section 1.b

Section 3.b

Section 1.c

Section 2.a, Section 3.a of [14]

Section 2.a, Lemma 3.10 of [14]

Section 2.a, Lemma 3.10 of [14]

Section 2.a, Lemma 3.10 of [14]

Section 2.a 
$\mathfrak{v}$

$\mathfrak{b}_{1} * \mathfrak{b}_{2}$

$U_{\gamma}$

$\mathbb{V}_{0}, \mathbb{V}_{1}$

$\chi \gamma$

$\mathfrak{p}_{\gamma, 0}$

$\left(b_{*}, \eta_{*}\right)$

$\left(A^{*}, \psi^{*}\right)$

$\mathfrak{L}_{*}$

$\vartheta_{\mathfrak{c}}$

$\Pi_{0}, \Pi_{\zeta}$ in the context of Equation (1-1)

$\mathbb{L}$ in Section 2

$\mathbb{L}$ in Section 3

$\mathbb{L} \perp$

$t$ in the context of Theorem 1.1

$\rho *$

$\mathbb{H}^{\perp}$ in the context of Theorem 1.1

$\mathfrak{b}_{* \zeta}$

$\mathfrak{e} \zeta$

$\left(b_{\zeta}, \eta_{\zeta}\right)$

$\mathrm{t}_{\zeta}$

$\mathfrak{L} \perp$

$\wp, \wp^{\dagger}$
Equation (2-1), Section 3.c of [14]

Equation (2-2)

Step 2 of Section 2.a, Step 1 in Section 3.a of [14]

Step 2 of Section 2.a, Step 3 in

Section 3.b of [14]

Step 2 of Section 2.a, Step 2 of Section 3.a of [14]

Step 2 of Section 2.a, Step 3 in

Section 3b of [14]

Step 2 of Section 2.a

Step 2 of Section 2.a

Step 2 of Section 2.a

Equation (2-4), Equation (2-6) of [14]

Step 4 of Section 2.a, Step 4 of Section 3.b in [14]

Step 4 of Section 2.a, Step 1 of Section 3.b in [14]

Part 2 of Section 3.a, Part 1 of Section 6.a in [14]

Step 4 of Section 2.a, Step 4 of Section 3.b in [14]

Equations (2-5) and (2-6)

Step 4 of Section 2.a, Step 1 of Section 3.a plus (4-8) in [14]

Step 4 of Section 2.a

Step 5 of Section 2.a, Lemma 3.10 of [14]

Step 5 of Section 2.a, Lemma 3.10 of [14]

Step 5 of Section 2.a

Step 5 of Section 2.a

Equation (2-19), Step 7 of Section 2.a

Equation (2-19), Step 7 of Section 2.a 
$\mathrm{D}, \mathrm{D}_{\gamma}$

$f_{v}, h_{\mu}$

$w$ : the function $s-\frac{1}{2}|z|^{2}$ on $\mathbb{R} \times \mathbb{C}$

$z_{\mathcal{E}}$

$\varsigma(t)$

$U_{\Sigma}$

$U_{\gamma_{-}}, U_{\gamma_{+}}$in Section 2

$U_{\gamma_{-}}, U_{\gamma_{+}}$in Section 3
$R$ : a large positive constant

Equation (2-19), Step 7 of Section 2.a

Equation (2-41)

Part 1 of Section 2.b

Part 1 of Section 2.b, Equation (4-8) in [14]

Part 1 of Section 2.b

Part 1 of Section 2.b

Part 1 of Section 2.b

Part 1 of Section 2.b

Part 2 of Section 3.a, Equation (5-3) in [14]

$N$ in the context of Theorem 1.1: the normal Part 2 of Section 2.b bundle to $\Sigma$

$\Sigma_{R}$ : the $|s| \leq 4 R$ part of $\Sigma$

$N_{1}$ : a fixed radius subbundle of $N$

$\omega_{\Sigma}$

$\omega_{a}=d s \wedge a+* a$

$\mathfrak{s :}$ the tautological section of $\pi^{*} N$

$z$ : the zero locus of $\omega_{\Sigma}$

$T$ : a tubular neighborhood of $Z$

$X_{Z}$

$X$

$\omega_{X}$

self-dual and anti self-dual spinor bundles

$\mathbb{S}_{I}, \mathbb{S}_{I, X}$

$K_{x}, L$

$\mathbb{S}_{I+}, \mathbb{S}_{E+}$

$\chi^{\Sigma}$ : the Euler characteristic of $\Sigma$

$k_{\Sigma}$

$\operatorname{deg}\left(N_{\Sigma}\right)$

$\left(A^{*}{ }_{x}, \psi^{*}{ }_{x}\right)$

$\mathfrak{b}_{\gamma_{-}}, \mathfrak{b}_{\gamma_{+}}$

$\mathcal{C}$ : the set of components of $\Sigma$

$U_{C}$ in Section 2

$U_{C}$ in Section 3
Part 2 of Section 2.b

Part 2 of Section 2.b

Part 2 of Section 2.b, Equation (2-57), Equation (2-58)

Part 2 of Section 2.b

Part 2 of Section 2.b

Part 2 of Section 2.b

Part 3 of Section 2.b

Part 3 of Section 2.b

Part 3 of Section 2.b

Part 3 of Section 2.b

Part 4 of Section 2.b

Part 4 of Section 2.b

Equation (2-60)

Part 4 of Section 2.b

Part 5 of Section 2.b

Part 5 of Section 2.b

Part 5 of Section 2.b

Part 6 of Section 2.b

Part 6 of Section 2.b

Part 6 of Section 2.b

Part 6 of Section 2.b, Equation (5-3) of [14]

Part 2 of Section 3.a, Equation (5-3) of [14] 
$\mathfrak{b}_{C}$

$\mathfrak{h}$

$\mathcal{E}_{c}$ : the set of ends of $C$

$e_{C}$ : an exponential map on $C$ 's normal bundle

$\rho_{\Sigma}$

$R_{\mathcal{E}}$

$\chi_{\mathcal{E}_{C}}$

$\left(A_{x}, \psi_{x}\right)$

$\mathbb{S}_{E_{-}}$: an anti-self-dual spinor bundle

$\Lambda^{2+}$ : the bundle of self-dual 2-forms

$\Delta_{A_{X}}$

$\mathfrak{D}_{E}$

$\left(A_{-}, \psi_{-}\right),\left(A_{+}, \psi_{+}\right)$

$\mathbb{H}_{X}, \mathbb{H}_{X}^{\perp}$

$\|\cdot\|_{\mathbb{H}, X}$

$\mathcal{K}_{1}^{2}$ in Section 2

$\mathcal{K}_{1}^{2}$ in Section 3

$t_{X}$

$\Pi_{X}$

$\mathbb{L}_{X}, \mathbb{L}_{X}^{\perp}$

$\mathcal{L}^{2}$ in Section 2

$\mathcal{L}^{2}$ in Section 3

$\sigma$ : a small positive constant

$p_{X}, p_{X}^{\prime}$

$\Delta$ in Section 2

$\Delta$ in Section 3

$\Delta_{E}$

$r_{\gamma}$ : This is $\left(\ell_{\gamma} / 2 \pi\right) r$.

$\left\{\sigma_{q}\right\}_{1 \leq q \leq m}$ : complex coordinates for $\mathfrak{C}_{m}$
Part 6 of Section 2.b, Part 3 of the proof of Lemma 6.3 in [14]

Equation (2-60)

Part 6 of Section 2.b

Part 6 of Section 2.b, Part 2 of

Section 4.a in [14]

Part 6 of Section 2.b, Equation (4-8) of [14]

Part 6 of Section 2.b, Equation (4-8) of [14]

Part 6 of Section 2.b

Part 6 of Section 2.b

Part 7 of Section 2.b

Part 7 of Section 2.b

Part 7 of Section 2.b, Equation (2-61)

Part 7 of Section 2.b

Part 8 of Section 2.b

Part 1 of Section 2.c

Equation (2-62)

Part 1 of Section 2.c

Part 2 of Section 3.a, Step 4 of

Section 5.b of [14]

Part 1 of Section 2.c

Part 1 of Section 2.c

Part 1 of Section 2.c

Part 1 of Section 2.c

Part 2 of Section 3.a, Part 7 of Section 6.a in [14]

Part 1 of Section 2.c, Section 4.c of [14]

Equation (2-64)

Equation (2-64)

Equation (3-11)

Equation (2-69)

Part 3 of Section 2.c

Part 3 of Section 2.c, Equation (1-5) of [14] 


$$
\begin{aligned}
& \rho(w) \\
& k_{R}^{>}, k_{R}^{<} \\
& \mathfrak{D}_{E *} \\
& \left(A^{\xi}, \psi^{\xi}\right) \\
& \mathfrak{h}(\xi) \\
& \mathfrak{q}(\xi) \\
& \mathfrak{d}_{\Sigma} \\
& \left(A_{\Sigma}, \psi_{\Sigma}\right) \\
& \mathfrak{D}_{\Sigma} \\
& \mathfrak{D}_{\xi} \\
& \mathfrak{e}_{q} \\
& \|\cdot\|_{\mathcal{K}_{1}^{2}} \\
& t_{\xi} \\
& \mathbb{H}_{\xi}^{\perp} \\
& \Xi_{\Sigma-}, \Xi_{\Sigma+}
\end{aligned}
$$

$\mathcal{E}_{4 R}, \mathcal{E}_{2 R}$

$\Pi_{\xi}$

$\mathbb{L} \frac{\perp}{\xi}$

$$
p, p^{\prime}
$$$$
\Lambda\left(\Theta_{-}, \Theta_{+}\right)
$$

$\operatorname{det}(\Delta)$ for a family, $\Delta$, of Fredholm operators $\mathcal{D}_{0-}$

$\mathcal{D}_{0+}$

$\mathcal{D}_{T \pm}$

$\mathcal{D}_{ \pm}$

$\mathcal{D}_{C ; T, T^{\prime}}$

$\mathcal{D}_{C} \diamond$

$$
n_{C \pm}
$$$$
\left(v_{C \diamond}, \mu_{C \diamond}\right)
$$

$n_{ \pm}$
Part 3 of Section 2.c

Part 3 of Section 2.c

Part 4 of Section 2.c

Part 1 of Section 3.a, Equation (5-15) in [14]

Part 1 of Section 3.a, Section 6.d of [14]

Part 1 of Section 3.a, Proposition 6.4 of [14]

Part 1 of Section 3.a

Part 1 of Section 3.a

Part 1 of Section 3.a

Part 1 of Section 3.a

Equation (3-1)

Part 2 of Section 3.a

Part 2 of Section 3.a, Equation (6-9) in [14]

Part 2 of Section 3.a

Part 2 of Section 3.a, Section 5.a of [14]

Step 2 of Part 1 of Section 5.a in [14]

Part 4 of Section 3.a

Part 4 of Section 3.a

Equation (3-11)

Section 3.b and (3-52)

Equation (3-7)

Equation (3-56)

Equation (3-57)

Part 3 of Section 3.b

Part 3 of Section 3.b, Equation (3-58)

Part 4 of Section 3.b

Part 5 of Section 3.b

Part 5 of Section 3.b

Part 5 of Section 3.b, Equation (3-64)

Part 6 of Section 3.b 


$\mathcal{D}_{T 0}$
$\mathfrak{D}_{\Sigma *}$
$\mathfrak{D}_{\xi *}$
$\mathcal{D}_{C *}$
$p, p_{*}^{\prime}$
$\Delta_{*}$
$\mathfrak{D}_{\Sigma ; T, T^{\prime}}$
$\mathfrak{D}_{ \pm}, \mathfrak{D}_{\Sigma} \diamond$

$\mathcal{D}_{T 0}$

Part 6 of Section 3.b

Part 7 of Section 3.b

Part 7 of Section 3.b

Part 8 of Section 3.b

Equation (3-67)

Equation (3-67)

Part 11 of Section 3.b

Part 11 of Section 3.b, Equation

\section{References}

[1] J-M Bismut, D S Freed, The analysis of elliptic families. I. Metrics and connections on determinant bundles, Comm. Math. Phys. 106 (1986) 159-176 MR853982

[2] F Bourgeois, K Mohnke, Coherent orientations in symplectic field theory, Math. Z. 248 (2004) 123-146 MR2092725

[3] S K Donaldson, P B Kronheimer, The geometry of four-manifolds, Oxford Math. Monogr., Oxford Science Publ., The Clarendon Press, Oxford Univ. Press, New York (1990) MR1079726

[4] R Gompf, private communication

[5] H Hofer, K Wysocki, E Zehnder, Properties of pseudoholomorphic curves in symplectisations. I. Asymptotics, Ann. Inst. H. Poincaré Anal. Non Linéaire 13 (1996) 337-379 MR1395676

[6] M Hutchings, An index inequality for embedded pseudoholomorphic curves in symplectizations, J. Eur. Math. Soc. (JEMS) 4 (2002) 313-361 MR1941088

[7] M Hutchings, C H Taubes, Gluing pseudoholomorphic curves along branched covered cylinders. I, J. Symplectic Geom. 5 (2007) 43-137 MR2371184

[8] M Hutchings, C H Taubes, Gluing pseudoholomorphic curves along branched covered cylinders. II, J. Symplectic Geom. 7 (2009) 29-133 MR2491716

[9] P Kronheimer, T Mrowka, Monopoles and three-manifolds, New Math. Monogr. 10, Cambridge Univ. Press (2007) MR2388043

[10] T Mrowka, A local Mayer-Vietoris principle for Yang-Mills moduli spaces, $\mathrm{PhD}$ thesis, University of California, Berkeley (1989)

[11] D Quillen, Determinants of Cauchy-Riemann operators on Riemann surfaces, Funktsional. Anal. i Prilozhen. 19 (1985) 37-41, 96 MR783704

[12] C H Taubes, Asymptotic spectral flow for Dirac operators, Comm. Anal. Geom. 15 (2007) 569-587 MR2379805 
[13] C H Taubes, Embedded contact homology and Seiberg-Witten Floer cohomology I, Geom. Topol. 14 (2010) 2497-2581

[14] C H Taubes, Embedded contact homology and Seiberg-Witten Floer cohomology II, Geom. Topol. 14 (2010) 2583-2720

[15] C H Taubes, Embedded contact homology and Seiberg-Witten Floer cohomology IV, Geom. Topol. 14 (2010) 2819-2960

Department of Mathematics, Harvard University

Cambridge, MA 02138, USA

chtaubes@math . harvard.edu

Proposed: Rob Kirby

Received: 15 November 2008

Seconded: Danny Calegari, Peter Ozsváth

Revised: 11 May 2010 